
14

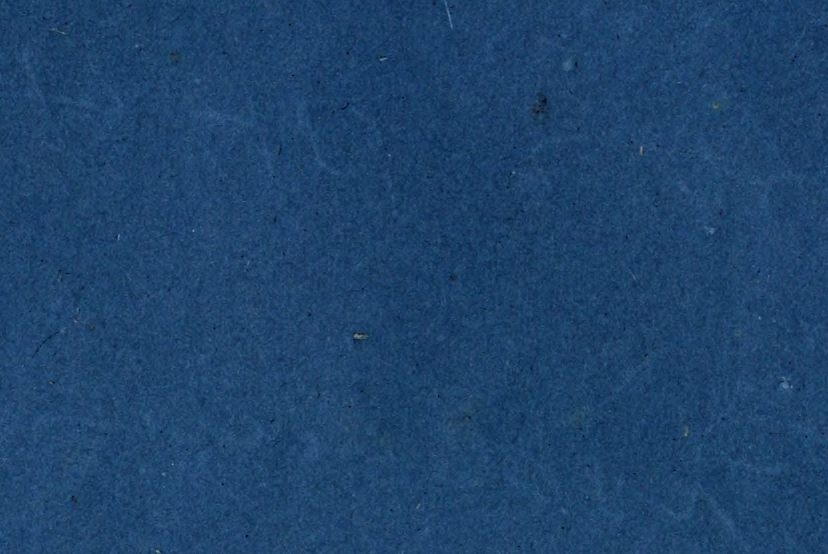

sises

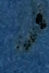






\section{PRODROMUS}

VI, ORAE BA T A VAE. 


\section{TYPIS DE BREUK \& SMITS.}




\section{PRODROMUS}

\section{FLORAE BAT A VA E.}

VOLUMEN II. PAKS IV.

(F'ungi.)

SUMTIBUS SOCIETATIS

APOD

JAC. HAZENBERG, Conneri Fil.

1866. 
2014091010399

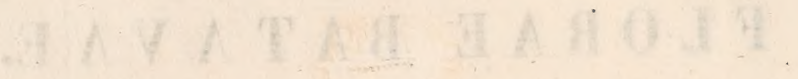

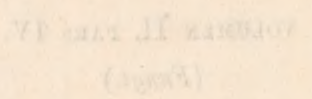

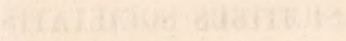

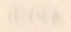

and 0 
Llet besluit om de bewerking der lagere Fungi van het llerbarium der Vereeniging voor de Flora van Nederland en zïne overzeesche bezittingen op te dragen aan een buitenlandsch correspondeerend lid, werd genomen op de jaarvergadering van 1860, toen het gebleken was, dat geen van de gewone leden der Vereeniging zich met die taak wilde of durfde belasten. De keuze van het Bestuur viel op den Heer G. D. We stendorp, officier van gezondheid bij het Belgische leger te Dendermonde, een man, die, door vele geschriften, als grondig mycoloog zich een welverdienden naam verworven had. Genoemde Heer had de groote welwillendheid, aan onze roepstem gehoor te geven, en zond ons zijn manuscript in Juli 1863 over.

Door omstandigheden, die het nutteloos zou wezen hier na fe gaan, werd het ter perse leggen van het manuscript des Heeren Westendorp tot in Augustus 1865 vertragegd. Do ondergeteekende, in dat jaar tot Voorzitter onzer Verecniging gekozen, spande terstond alle pogingen in om het verzuim te herstellen, en verheugt er zich in dat hij thans, hoezeer dan ook een vol jaar later, het laatste stuk van den Prodromus zijnen medeleden kan aanbieden.

De Heer Westendor p heeft de goedheid gehad, de laatste proef van alle vellen na te zien.

De ondergeteekende acht zich geroepen, aan voornoemd cor= respondeerend lid onzer Vereeniging onzen hartelijken dank over te brengen voor de hulpvaardigheid, ons bewezen.

Amsterdam Aug. 1866. 

La détermination des champignons inféricurs (Gasteromycetes, Pyrenomycetes, Hyphomycetes et Coniomycetes) de l'herbier de l'Union, nous a présenté de grandes difficultés, en ce que beaucoup de Specimen qui s'y trouvaient, étaient surannés, ayant été colligué depuis un grand nombre d'années; que d'autres avaient été détruits par les insectes on par l'humidité; que quelques uns en assez grand nombre avaient été récoltés lorsque la plante commencait seulement à se développer ou bien lorsqu'elle était déjà dans un état de développement trop avancé; conditions qui ne permettent pas de déterminer avec certitude. Enfin que pour la masse, certaines indications qui facilitent les recherches, telles que le nom de la plante qui sert de support, l'endroit et l'époque de l'année où elle à été trouvée, le nom de la personne qui l'a découverte, etc., etc., manquaient ou étaient fautives. Néanmoins et mạlgré toutes les difficultés que nous avons eu à surmouter, nous avons été assez heureux de pouvoir déterminer, avec plas ou moins de certitude, environ sept cent espèces!

Nous aurions pu nous dispenser de citer plusicurs tribus, tels que les Phylleriacei, les Destructorii, voire même les Cylisporei, parceque leur autonomie a été contestée par plusieurs auteurs. Mais ayant été chargé seulement de faire le relevé de ce qui se trouvait dans l'herbier de l'Union, nous avons cru bien faire en mentionnant dans ce Prodrome, tout ce qui s'y trousait, en laissant aux futurs auteurs de la flore 
Neriantabe ie suit deliminer ec que di selenee suta reconma amme ne devant filus être ensidér comme tribu, sene w espèce distinctes.

Cette partie de la mycologie ayant été entièrement négyligé jar les auteurs qui se sont occupés de l'énumération des plantes ite la llore indigène, nous n'avons pu consulter aucun ouvrage Nécrlandais, sauf deux opuscules de Dozy et Molkenboer, ainsi que quelques notes manuscrites de Mr. Franquinet, concernant les plantes Cryptogames des environs de Maastricht. Quand aux autres ouvrages que nous avons du consulter pour la détermination des espèces et qui se trouvent cilés dans cette dernière partie du prodrome, nous en donnerons ici la liste, avec les abbréviations employés entre parenthèses:

Bonorden. IIandbuch der algemeinen Mycologie. (Bon. Ilandb.)

Chevallier. Flore des environs de Paris. (Chev. fl. Par.) Corda. Icones fungorum. (Cord. Ic. fung.)

Desmazières. Plantes Cryptogames de France, $1^{\text {re édit. }}$ Fascicules 1 ì 44. (Desmaz. Pl. Crypt. Fr. $1^{\text {re }}$ Ed.)

Plantes Cryptogames de France. Nouvelle édition. Fasc. 1 à 16. (D e $\mathrm{smaz}$. Pl. Cr. Fr. nouv. Ed.)

1) Notices 1 à 24 sur les Cryptogames nou. velles ou inédites de la flore Française, extraites des mémoires de la -Société Royale des Sciences de Lille, et des Annales des Sciences naturelles (Partie Botanique des ${ }^{10}, 2^{\circ}$ et $3^{e}$ séries.) (Desmaz. Not. Crypt. Nouv. de Fr.)

5) $a$ y filolkenbocr. Novae fungorum species in Belg. Septent. nuper detectae. (Dz. et II b. Nor. (ung.)

" Bijdragen tot de flora Cryptomamica virm Ne- 
derland in het Tijdschrift voor natuurl, Gesch. en Physiologie $\mathrm{X} \mathrm{L}^{\mathrm{e}}$ deel. ( $\mathrm{D}$ z. et $\mathbf{M} \mathrm{b}$, Bijdr.)

Duly.

(I

Fée.

》)

)

$\infty$

K ic $\mathrm{k}$.

"1

Libert.
Botanicon gallicum. (D u by Bot. gal.)

IIémoire sur la tribu des Hystérinées. Extr. du T. XVI des Mlémoires de la Société de physique et d'histoire naturelle de Genève. (D a b y. Hém. Hyst.)

Mémoire sur le groupe des Phyllériées et notamment sar le genre Erineum. (Fée Phyll.)

Fries. Elenchas fungoram. (Fr. Elench.)

Epicrisis fungorum. (Fr. Epicr.)

Summa vegetabilium Scandinaviae (Fr, Samm. Veg.)

Systema mycologicam (Fr. Syst.)

Léveillé. Champignnons du sausée de Paris. Extraits des Annales des sciences naturelles, $2^{\circ}$ Série T. V. pag. 111 et 249. (Lév. Champ.)

Fragments mycologiques. Estr. des Anr. des sc. nat. 2e Séric. (Lé v. fragm. myc.)

Organisation : et disposition méthodique des espèces da genre Erysiphe. Estr. des An-

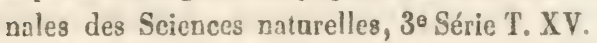
p. 109. (Lé ซ. Erys.)

Disposition métliodique des Urédinées. Extr. des Annales des Scicnces naturelles, $2^{\circ}$ Sér. T. II. p. 1).

Flore cryptogamique des environs de Louvain. ( $\mathrm{Kx}, \mathrm{fl} . \mathrm{Cr}$. Louv.)

Recherches sur la tore cryptogamique des Flandres. 5 Centaries. Extrait des mémoires de l'Académie Royale des Sciences de Belgique. ( $\mathrm{K} \times$. Rech.)

Plantae cryptogamicae arduennae. ( $L$ ko Pl. Crypt.) 
Montagne. Sylloge gen. specierumque eryptogemorum. (11lont. Syll.)

Persoon. Synopsis fungorum. (Pers. Syn.)

Rabenhorst. Deutschland's Kryptogamen-Flora (Rabenh.) "Herbarium vivum mycologicum. (R a benh. Herb. myc.)

" Fungi Europaei. (R a b e $\mathrm{n}$ h. fung. Eur.)

Wallroth. Compendiam florae germanicae. (Wallr Comp.)

Westendorp. Herbier eryptogamique Belge. (West. H e r b. crypt.)

" Notices 1 à 8 sar les Cryptogames nouvelles ou inédites de la flore Belge. Extr. des Bulletins de l'Académie Royale des Sciences de Belgique, et des Bulletirs de la Société Royale de Botanique de Belgique. (W e s t. Not. crypt. inéd. fl. B.)

Ayant rencontré, en examinant les collections, des espèces ot des localités qui n'avaient pas été mentionnées dans les rrois premières parties da second volume du Prodrome, nous les avons réunies en tête de cette dernière partie sous le titre d'Addenda.

\section{WESTENDORP,}

Médecin de l'armós Belge, Vice Président de la Société Royale de Botanique de Belgique.

Termonde, le 12 Juillet 1863. 


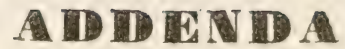

\section{ad Wol, II IPart, I, en $3 \mathrm{am}$.}

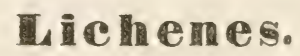

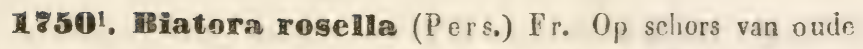
boomstammen. (Rabenh. p. 94. - Basidia rosella De Not.; Körb. Syst. Lich. Germ. p. 185)

In Herb.: Holland, v. d. Ende (v. s.)

1511. Verrucaria nítala $b$, nitidella Flörk. Op oude Berkenstammen, (Flörk, Deutschl. Lich. no. 10; West. Herb. Cr. Beige no. 117)

In Herb. : Duinkant te Walcheren, v. d. B. (v. vo et s.)

18121. Werrucaria einerea $\mathrm{Kx} .0_{p}$ schors van oude Wilgen, alsook op schuttingen. ( $\mathrm{Kx}$. Rech. sur la Fl. Cr. des Fland. 2 Cent. p. 24; West, Herb. Cr. Belge $\left.n^{\circ} .22\right)$

In Herb.: Amsterdam, v. d. S. L a c. (v. v. et s.)

18122. Verrucaria gemmata Ach. $0 p$ schors van oude Beuken en Esschen. (R a benh. P. 12; West. Herb. Cr. Belge, $n^{\circ}, 23$ )

In Herb.: Holland. 


\section{1}

2061 ${ }^{1}$. Rostoc tuberesulosum $\mathbf{K} \ddot{t} t z$. Op overstroonde koemest in polders.

Subinundatum; phycomate olivaceo-atro, tuberculato, compacto, duro; trichomatibus flexuosis, laxe implicatis, aequalibus, dilute aerugineis; articulis granulosis, oblongis, angulatis, dimidiatis; spermatiis ellipticis, rarissimis; peridermide luteo-fuscescente. (K ü tz. Sp. Alg. p. 298; K ü tz. Tab. phyc. II. T. 5. f. V.)

In Ilerb.: Zuid-Beveland (Goes) v. d. B. (v. s.)

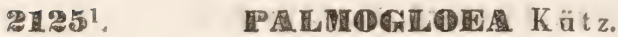

Stratum gelatinosum difforme indeterminatum, ex celo lulis sparsis polygonimicis in substantia gelinea nidulantibus compositum. (Kütz. Sp. Alg. p. 228)

TiP. produberans $\mathrm{K}$ ü $\iota$. $O_{p}$ vochtige aarde, mossen en boomtronken in bosschen. (Kütz. Sp. Alg. p. 228; Coccochloris protuberans Spreng.; Rabenh. p. 60)

In Herb.: Zuid-Beveland, v. d. B. (v. vo et s.)

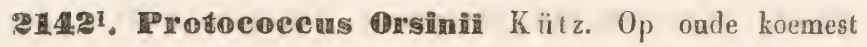
op vochtige plaatsen.

Strato crustaceo e rubro-aurantiaco, aurantiaco.cinna* momeo et olivaceo-lutescente variegato; cellulis simplicibus globosis, variis, membranala crassiuscula achromatica laevissima, nucleo solido aurantio subhomogeneo, centro granuloso.

Diam. 1/400-1/70"”. (Kütz. Sp. Alg. p. 202, )

In IIerb.: Zuid-Beveland, v. d. B. (v. v. et s.)

\section{Tu}

26301. Cantharellus bryophilns F r. Op Hypnum Sto- 
kesii. Vere. (Fr. Syst. I. p. 325; Rabenh. p. 445) In Ilerb.: Amsterdam, v. d. S. Lac. (v. v. et s.)

2636'. Marasmius Spodoleucus Berk. et Broome. Op doode afgevallen takjes in de bosschen. Aut. Vere. Conchiformis, resupinatus, margine liberato, supra cincreus, subfurfuraceus; stipite prorsus nullo; hymenio albo, lamellis paucis; interstitiis laevibus. Berk. et Br. (R abenh. Fung. Eur. $n^{\circ}$. 205)

In Herb.: Naaldwijk, v. d. Tr. (

28191: Cyphella ampla $L$ ev. $O_{p}$ afgevallen takken en houtsplinters. Aut. Vere.

Receptaculum sessile membranaceum cupulatum alo bum tomentosum, intus venosum cervinum, margine tenui integro. (Lev. fragm. myc. in ann. Sc. nat. 3 Sér. T. IX. pag. 126; Cyphella Coemansii $\mathrm{h}$ aben h. Fung. Eur: $n^{\circ}, 209$ )

In llerb.: Amsterdam, v. d. S. Lac. (v. vo et $s_{\text {. }}$ )

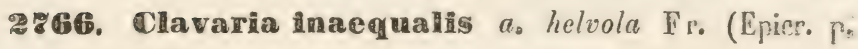
577) In bosschen tusschen het gras.

In Herb.: Zuid-Beveland, r. d. B. (v. v. et $s_{\iota}$ ) b. aurantiaca $\mathrm{Fr}$. Il. Op dezelfde plaatsen.

In Herb. : Zuid-Beveland (Goes) v. d. B. (v. v. et s.)

2\%851. Exidia glandulosa $a$. papillata $\mathrm{Fr}$. Op rotend hout. Ilieme. (F r. Syst. II. p. 225)

In Herb.: Zuid-Bereland, v. d. B. (v. s.)

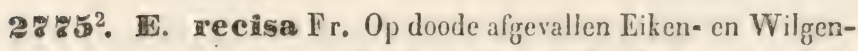
takken. Hieme. (F r. Syst. II. p. 223; R a ben h. p. 313)

In Herb.: Groningen, de Haan; Leiden, Meppel, D z. et $\mathrm{Il}$ b.; Harendermolen, Strat. (v. v. et s.)

28\%81. Tremella alloida $\mathrm{Fr}$. Op rottend hout, bijzonder van Eiken en Beuken. Vere. (Fr. Syst. II. p. 215; Rabenl. p. 311)

In Herb.: 't Haagsche bosch, WItw.; Zuid-Beveland, v. d. B. (v. vo et s.) 
b. effusa Alb. et Schw. Op rottende Populierschors. (Trem. gemmata Lev, in Herb. Unio?)

In Herb. Zuid-Beveland, v. d. B. (v. s.)

2\% 8 82. Tr. auranti̊a Schwein. Aan oude palen. Hieme. (Fr. Syst. II. p. 213)

In Herb.: Zuid-Beveland, v. d. B. (v. s.)

28 883. Tr. Iutescems $\mathrm{Fr}, 0 \mathrm{p}$ rottend hout. Hieme. (Fr. Syst. II. p. 213 ; Rabenh. p. 311)

In Herb.: Goes, v. d. B. (v. s.)

28\%8. Tromenterica $R$ etz. Adde:

In Ilerb.: Leiden, Dz. et M bs ; Harderwijk, J u n g h. ;

Leiden, $\mathrm{W} t \mathrm{t}$.

28.8.

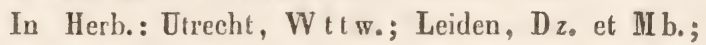
Haastricht, Fran q.

2\%53. Hacr. stillatus $\mathrm{N}$ e es. Adde:

In Herb.: Harderwịk, J ungh.; Leiden, de II a a n, W ttw.; Maastricht, F ranq.

28831.

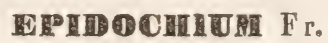

Stroma e ceraceo-corneum, superficic impolita ; sporis continuis subpyriformibus, sporophoris suffultis. (Fr. Summ. Veg, Sc. p. 471.)

Ep. NIartensil West. $O_{p}$ doode afgevallen Lindetakken.

Réceptacles épars, immergés, aplatis, bleu-noirâtres, arrondis, ne dépassant jamais un mill. de diamètre. Basidies filiformes, rameuses, dichotomes, hyalines, à sporidies terminales uniques. Sporidies ovale-allongées , transparentes, d'un beau bleu, mesurant $3 / 100$ es de mill. de longueur sur $1 / 100$ de mill. de largeur. (West. 4 e not. Crypt, nouv. de la fl. B. p. 13. $\mathrm{n}^{\circ}, 56$ T. I. f. 6.)

In Herb.: Leiden, Dz, et $13 \mathrm{~b}$. (v. v. et s.) 
2851. Cylindrocolla Urticae $B$ on. Adde.

In Herb. : Groningen, de $\mathrm{H}$ a a $\mathrm{n}$; Leiden, $\mathrm{W}: \mathrm{t}$ w; Amsterdam, v. d. S. Lac.

2866. Podisoma Gymmosporangium Bon. Adde:

In Herb.: Leiden, in 's Rijks Plantentuin, W 1 w.; Aalsmeer, v. d. S. L a c.; Amsterdam, v. d. S. Lac.

281D1. Peziza fascicularis $\mathbf{A}$ lb. et $\mathrm{Schw}$. Op diode Elzen- en Populiertakken. Aut. Vere. (F r. Syst. II. p. 75; Rabenh. p. 367)

In Ilerb.: Haarlein, Dz. et II b.; Zuid-Beveland, v. d. B. (v. v.)

29221. TPez, brunneo-atra Desmaz. $O_{p}$ de aarde op vochtige plaatsen; ook op verrot hout. Vere.

Sessilis, solitaria, majuscula, subplana, integra, carnosa, fragilis, glabra, brunneo-atra; ascis maximis, eylindricis; sporidiis ovoideis, subhyalinis. (Desmaz. PI. Crypt. de Fr. ${ }^{\circ}$. 826)

In Herb.: Leiden, Dz. et II b. (v. s.)

28281. Pez. ambrosa $\mathrm{Fr}, \mathrm{Op}$ de aarde tusschen mossen. Aut. Vere. (Fr. Syst. II. p. 85; R a ben h. Fung. Eur. $\mathrm{n}^{\mathrm{0}}$. 217.)

In Herb.: Zuid-Beveland (Goes), v. d. B. (v. s.)

2831. IPeziza cilliaris $\mathrm{Fr}$. $0 p$ doode afgevallen likenbladen. Vere. (Fr. Syst. II. p. 89; R a ben h. p. 363) In Herb. Leiden, $\mathbf{D} z$. et $\mathrm{II}$ b. (v. vo et s.)

28321. TPez. eiversicoler $\mathrm{F}$ i. 0 p unden verdroegden koedrek. Aut. (Fr. Syst. II. p. 88; R a benh.p. 363)

In Herb. Zuid-Beveland, v. d. B. (v. v. et s.)

as 101. Heer. sanguinea l'ers. Op rottend hout. Hieme. (Fr. Syst. II. p. 110; Rabenh. p. 356)

In Herb.: Utrecht, Dz. et II b. (v. s.)

28451. rex. inflexa $\mathrm{Bolt}$. Op dinde stengels ran fironte kruidgewassen. Vere. (Bolt. Ilalif. III. p. 106. Tab. 
106. f. 2 ; Fr. Syst. II. p. 120 ; Rabenh. p. 353)

In Herb.: Utrecht, v. d. S. Lac. (v. v. et s.)

208452. Pez. striata $\mathbf{N}$ ees. 0 p doode stengels van Brandnetels. Vere. (Fr. Syst. II.p. 122; R a ben h. p. 352; Rabenh. Fung. Eur. n² 221)

In Herb.: Warmond, de $\mathrm{H}$ a a $\mathrm{n}$ in Herb. De V r. (v. s.)

28453. Pez. albida $R o b .0 p$ doode afgevallen bladstelen van Esschen. Vere. Aestate.

Sparsa aut laxe gregaria, stipitata, fragilis. Cupula minuta, eburnea, acetabuliformis, dein plana, margine integerrimo; stipite crassiusculo, basi rufo; ascis clavatis; sporidiis hyalinis, oblongis, obtusis, subcylindricis; sporulis 2-3. (Rob. in Desmaz. 19e not. Cr. de Fr. p. 28; Desmaz, Pl. Cr. de Fr. no. 2004)

In Ilerb.: 't Bosch te Zalk, R. Bond. (v. v. et s.)

28501. Mez. Habi var. herbicola Rabenh. Op doode stengels van groote kruidgewassen. Vere. (Rabenh. Fung. Eur. n". 218)

In Herb.: Utrecht, v. d. S. Lac. (v。 s.)

Obs. Peziza Rubi ei herbarnm proxima, sed differt a priori colore laete- et fulvo-sulphureo nec non jam loco natali, a posteriori stipite breviore verruciformi.

28511. Teziga lacustris $F$. $0 p$ doode halmen van Scirpus lacustris. Hieme. Vere. (Fr. Syst. II. p. 143; Rabenh. p. 347)

In Herb.: Amsterdam, D z, et Ill b. (v, vo et s.)

28522: Pez. neglecta $\mathrm{Lib}$. Op doode stengels van Brandnetels. Vere.

Erumpenti-superficialis, cupulis primum concavis demum planiusculis, marginatis; margine fusco; disco aurantio-rubro; ascis clavaeformibus paraphysibus immixtis; sporidiis ovatis. (Lib. Pl. Cr. Ard. no. 29. Pcz. fusarioides Berk。; Desmaz. PI. Cr。 fr。 $\mathrm{n}^{\circ}$ 1063)

In Herb.: Leiden, $\mathbf{D}_{z_{\text {。 }}}$ et $\mathbf{M} b_{\text {b. }}$ Zuid-Beveland, v. d. B. (v. v, et s.) 
Obs. Inter Cylindrocollam Urticae frequenter occurit cum quo facile confundi potest; sed notis allatis valde differt.

2552. Hez. Hederae Lib. $0_{p}$ siervende en donde bladen van Hedera Helix. Per annum.

Dense sparsa, innato-erumpens, orbicularis, pallidonigricans, laciniis quatuor epidermidis cincta, disco submolli viridi-olivaceo; ascis clavatis; sporidiis ovoideis hyalinis. (Lib, Pl. Cr. Ard. nº 229 ; Pez, insidiosa Desmaz.)

In Herb.: Leiden, Dz. et II bo; Zuid-Beveland, v. d. B. (v. v. et s.)

25524. IPez. sphaeroides $v$. Lychnidis Desmaz. Op doode stengels van Lychnis dioica en sylvestris. Vere. (Desmaz. P1. Cr. de Tr. ${ }^{\circ}$. 174. Sine descr.)

In Herb.: Leiden, Dz, et $M$ b. (vos.)

28525. Pez. graminis Desmaz. Op doode bladen en halmen van verschillende Grassoorten, Vere.

Sparsa, erumpens, sessilis, ceraceo-mollis, minima, glabra, concava, umbrina; disco griseo-pallente; margine villosiusculo albo; ascis elavatis, minutis; sporidiis oblongis, linearibus. (Desmaz. 8 e not. Crypt. de Fr. p. 5; Desmaz. PI. Cr. de Fr. no. 1066)

In Herb. Amsterdam, v. d. S. Lac. (v. v. et s.)

28526. Pezima arenivaga Desmaz. Op rottende bladen van Psamma arenaria. Hieme. Vere.

Foliicola, erumpens, minuta, sparsa, ceraceo-mollis , glabra, sessilis, junior globosa, adulta plana, extus fulva, margine elevato tumidiusculo, fulvo, sicco nigro. Disco aquoso, suballido, sicco bruuneo. Ascis clavatis, subeylindricis, inter paraphyses simplices esectis; spo= rulis octonis, oblongo-ovoideis, hyalinis, utrimque obiusis. (De s maz. $20^{\mathbf{e}}$ not. Cr. inéd. de Fr. pag. 19)

In Herb.: Hollandsche duinen, v. d. B. (v. s.)

2S527. Hez. arduenacmsis U ont. Op duode wildo Rucentakken, Ilieme. Vere. 
Sparsa, crassiuscula. Cupula sessilis, planiuscula, marginata, flavo-cerina; disco pulverulento, margine demum evanescente; ascis clavaeformibus; sporidiis oblongatis biseptatis. (II ont. Syll. gen. spec. Crypt. p. 186. Patellaria Rubi Lib. Pl. Ci. Ard. $n^{\circ}$. 231)

In Herb.: Amsterdam, v. d. S. L a c.

d. Patellea. (Sessilis, ceraceo-lenta, marginata.)

29528, Pez. sallicaria Pers. $O p$ rottend hout in holle Wilgentronken. Hieme. Vere. (P ers. Myc. Eur. I p. 308; Pez. flexella b. salicaria Fr. Syst. II. p. 152)

In Herb.: Willemspulder, $\mathbf{D z}$. et M $\mathbf{~ b}$; Amsterdam, v. d. S. Lac.

2854!

פTICTHS P e r s.

ฐ๕. radliata Pers. Op een ouden stervenden Lindestam. Aut. Aest. (Fr. Syst. II. p. 194; R abenh. p. 333) Variat:

a. major (Desmaz. Pl. Crypt. de Fr. no. 1532)

In Herb.: Zuid-Beveland (Goes), vo d. B. (v. v. et 8.)

Discus innatus, applanalus, maculaeformis, immarginatus, epidermide secedente tectus; sporae simplices, ascis clavatis recepta. (Fr. Summ. Veg. p. 373)

N. valvata IIont. Op doode bladen van Psamma arenaria. Aut. Hieme.

Innata, elliptico-oblonga, tecta, demum epidermide circumscissa elapsa nuda; hymenio (disco) submarginato nigro; ascis filiformibus sporas golobosas uniseriatas foventibus, (HI ont. Syll, gen. spec. Crypt。p. 198; 
Stictis valvata MI ont. Ann. des Sc, nat, 2 Sér. T. VI. p. 337)

In Herb.: Hollandsche duinen, vo d. B. (v. v. et s.)

28551. THPHANISTode。

T. conspersa $\mathrm{Fr} . O_{\mathrm{p}}$ afgestorven takken van Sorbus Aucuparia, Pirus Malus, ete. Per anuum. (Fr. Syst. II. p. 175; $\mathbf{R}$ abenh. p. 336)

In Herb.: Amsterdam, 0 udem. (v. v. et s.)

28552.

\section{CATINULA.}

C. turgida Desmaz. Op afgestorven schor's van Corylus Avellana. Aest. Aut. (Excipula turgida Fr. Syst. II. p. 189; Rabenh. p. 152)

In Herb.: Hulst, West. (v. v. et s.) 


\section{Dral. Gasteromyceles.}

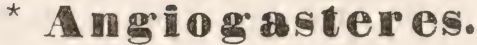

\section{Trib. Phalloideae.}

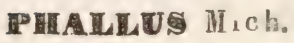

2856. Th. impudieus $L$. In bosschen op vochtige en moerassige phatsen. Jul. Aug. (F r. Syst. II. p). 283; R abenh. p. 307)

In Ilerb.: Noordwijk aan zee, Dz. et $11 \mathrm{~b}$. Bovendien: Bosch van Wolfhezen, Un. 1847; Velp, Un. 1849; Nịmegen, A be l.; Rolterdam, 0 u de em. (v. v. et ic.)

9858. Wh. caninus Huds. In bosschen aan en bij vermolmde tronken (van Corylus) Aut. (F r. Syst. 11. p. 285; Rabenh. p. 307)

In Herb.: Abtspoel bij Leiden, Dz. et 11 b. Bovendien: Zeist, 0 udem. (r. v.)

\section{Trib, Clathracei. \\ CLATHES Hich.}

2858. C. cancellatus L. $0 p$ vochtige platsen, aan mocrassen en waterkanten. (Fr. Syst. II. p. 288; R a ben h. p. 306)

Leiden (eenmaal aan den waterkant in den Hortus academicus, $\mathbf{j} z$, et $\mathbf{H} \mathrm{b}$.) ( $\left.\mathrm{v}_{\text {。 }} \mathrm{v}_{\mathrm{*}}\right)$ 


\section{Trib. Nidulariacel.}

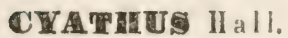

2859. C. striatus Willd. In bosschen en op beschaduwde plaatsen, tusschen en aan afgevallen bladen, takken, enz. Aut. (Rabenh. p. 306; Nidularia Fr. Syst. II. p. 298; Dz. et II b. in Bijdr. XI p. 392)

In Herb.: Leiden, $\mathbf{D}$ z. et $\mathrm{Ml}$ b.; Renkom, Buse. Bovendien: Westland, vod.Tr. (v. v. et s.)

2860. C. Dila Pers. Aan takken, spaanders, ruigten, enz., op vochtige plaatsen. Oct. Nov. (R a benh. p. 305; Cyath. vernicosus Dec.; Nidularia campanulata Fr. Syst. II. p. 298; Dz. et H b. I. I. p. 392)

In Herb.: Leiden, $\mathbf{D}$ z. et $\mathrm{Ml} \mathrm{b}_{\bullet}$; Amsterdam, v. d. S. Lac; Bosch te Zalk, R. Bond.; Zuid-Beveland, v. d. B. (v. v. s. et ic.)

2861. C. Crucibalum $\mathrm{H}$ of $\mathrm{fm}$. Op rottende Dennenplanken en palen. Aut. (R a benh. p. 305; Nidularia Fr. Syst. II. p. 299; Dz. et $M$ b. I. I. p. 392)

In Herb.: Leiden en Haarlem, Dz. et M b.; Groningen, Strat.; Amsterdam, v. d. S. Lac; Wassenaar, v. d. B. (v. v.)

\section{Trib. Carpobolei.}

\section{SPHEAREHOLUS Tode.}

2862. 5tellatus Tode. Aan hontzaagsel, spaanders, enz., op vochtige plaatsen. Aut. (Rabenh. pag. 303; Fr. Syst. 11. pag. 309)

In Herb.: Leiden, in den Hortus academicus, Dz. et N1 b.; Utrecht, v. d. S. Lac. (v. v.) 


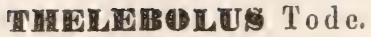

2863. T. stercoreus Tode. 0 p ouden koemest. Aug. Sept. (Fr. Syst. II. p. 307; Rabenh. p. 304)

In Herb.: Zuid-Beveland, v. d. B. (v. s. et ic.)

\section{* * Triclomycetes.}

\section{A. Trichogasteres.}

Trib. Geastrides.

GLATTER Mich.

2364. G. hygrometricus Pers. Op zandige heuvelen in Denuenbosschen. Aut. (R a benh. p. 301 ; Fr. Syst. III. p. 19.)

In Herb.: Wageningen, Buse. (v. v. en s.)

2865. Cr. rufescens $\mathrm{Fr}_{\text {}}$ In bosschen in zandige streken. Aut. (F r. Syst. III. p. 18; Rabenh. p. 302)

In Herb.: in 't Haagsche bosch, B isschop; Ilaarlem, Dz. et $M$ b. (v。 vo)

2866. F. mammosus $\mathrm{Fr} . O_{p}$ gelijke plaatsen als de vorige. Aut. (Fr. Syst. III. p. 17; R a ben h. p. 302; Fl. Bat. tab. 750,1$)$

In Herb.: Haarlem, D z. et II b. Bovendien: Westland, v. d. Tr. (v. v. et ic.)

286\%. F. fimbriatus F r. $0 p$ bedekte platsen up sehralen zandgrond. Aut. (Fr. Syst. 111.p. 16; R a ben h. p. 302) In Herb.: Haarlem en 's Hage, Dz. et II b. (v. v.)

\section{Trib. Lycoperdacei.}

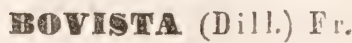

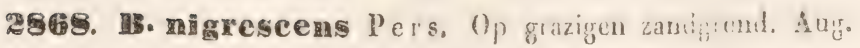
Septo (Fro Syst. III. p. 23; Raben h。 p. 301) 
In Herb.: Haarlem, Dz. et II b.; Zuirl-Beveland, v. d. B. Bovendien: Westland, v. d. Tr. (v. v. et S.)

2569. Plumbea Pers. $0_{p}$, relijke plaatsen als de vorige, in de duinen. Haj. Jun. Sept. (Fr. Syst. III. p. 24; Rabenh. p. 300)

In Herb.: Haarlem en Leiden, $\mathbf{D} z$, et $\mathbf{H l}$ b. ; Wicringen, v. d. S. La c.; Walcheren, vo d. B.; Beuningen en Weurt, A bel. Bovendien: Westland, v. d. Tr. (v. v. et s.)

\section{Z}

2880. L. piriforme $R$ upp. In bosschen, enz, op zandgrond (soms aan vermolmde tronken) groote zoden vormend. Vere. Aut. (Fr. Syst. III. p. 38; R aben b. p. 298)

In Herb.: Leiden en Wassenaar, $\mathbf{D}$ z. et $\mathrm{II}$ b. ; Kampen, R. Bond.; Zwake, v. d. B. (v. vo et s.)

2881. I. gemmatum B ats ch. In bosschen en op heschaduwde plantsen in zandige streken. Aest. Aut. (Fr. Syst. III. p. 36; Rabenh. p. 298) Variat.

u. excipuliforme S e op. In Ilerb.: Leiden, $0 \mathrm{udem}$; Wassenaar, D z, et Ml b. Bovendien: Westland, v. d. Tr. (vo v.)

B. perlatum Pers. In Herb.: Leiden, $\mathrm{D}_{\mathrm{z}}$. et $\mathrm{MI} \mathrm{b}$.; Zuidwijk hị Leiden, v. d. S. Lac. Bovendien: Westland, v. d. Tr. (v. vo et s.)

$\gamma$. echinatum Pers, In llerb.: Leiden, Dz. et $\mathbb{I I b}$. ; Benkom, Buse. (v. v.)

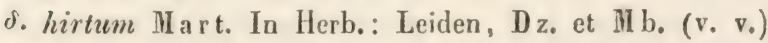

\&. furfuraceum $\mathrm{Fr}$. In Herb。: Leiden, $\mathrm{Dz}$. et $\mathrm{Mb}$. Bovendien: Nijmegen (Weurt), A bel. (v. vo et s.)

$\zeta$. papillatum S ch a eff. In Herb.: Westland, v. d. Tr. (v. S.)

2889. C. saccatum FI. D a n. $0_{p}$, heschaduwde plaatsen, in bosschen, enz., in zandige vochtige streken. Aut。 (Fr. Syst. III. p. 35; Rabenh. p. 299) 
In Ulerb.: Voorschoten bij Leiden, $\mathrm{Dz}$, et $\mathrm{H} \mathrm{b}$. Bovendien: Weurt bij Nijmegen, A bel. (v. v. et s.)

28\%3. 耳. Howista Linn. In weilanden en op grazige gronden. Aest. Aut. (Fr. Syst. 111. p. 29; R a be n h. p. 299) In Herb.: Leiden, $\mathbf{D}$ z. et $\mathbf{M l}$ b. ; Amsterdam, v. d. S. L a c.

9881. L. coelatum Bull. In weilanden en op grazige plaatsen en zandgronden. Aest. Aut. (F r. Syst. III. p. 32 ; Rabenh. p. 300)

In Herb.: Voorschoten bị Leiden, D z, et II b.; ZuidBeveland, v. d. B.; Heerwijk bij Nijmegen, A bel. (v. v. et s.)

28\%5. 耳. pusillum Batseh. Op grazige platsen aan dịjken en wegren, in bosschen en weilanden. Sept. Oct. (Fr. Syst. III. p. 33; Rabenh. p. 300)

In Herb.: Leiden, Dz. et M b. ; Zuid-Beveland, vo d. B. Bovendien: Westland, v. d. Tr. (v. v. s. et ic.)

2686. I. molle Pers. $O p$ de aarde in Dennenbosschen. Aat.

Subparvum, saepe solitarium, peridio turbinato piriformi rufescente, superne papillis caducis furfuraceis minutis acutisque confertissimis obruto, caule glabriusculo. Pers, in Chev. fl. Par. I. pag. 353. Tab, 10 fig, 2 。 In Herbs: Renkom, Busc. (v. vo et s.)

28\%\%. 耳. hiemale Bull. Op grazige platsen. Aut.

Paulo minus, peridio subsphaerico regulari glabrius. culo ex albido fuscescente, verrucis in junioribus minutis confertis demuin sparsioribas subfurfuraceis caducis, eaule subobeso plicato. Buil. Champ. Tab. 72 et Tab. 475. E (Chev. fl. Par. pag. 355)

In Herb: Renkom, Buse. (v. v. et s.)

2989. 更. turhoinatum a. lividum Pers. Op grazige plantsen in de duinen. Aut. Vere.

Turbinatum basi crassa productam brunneum niti- 
dum, verrucis exiguis persistentibus ( $P$ ers $\mathrm{s}$ in Joum. Bot. 1809. Tom. II. Tab. 1 fig. 3.4.5; West. llerb。 Crypt. Belg. $n^{\circ}$ 1264)

In Herb.: Katwijk, W $t$ tw. (v. s.)

\section{TULOTIIA Pers.}

2889. Thammosumn (Hich) Fr. Op mosachtige platsen in de duinen. Aut. (Fr. Syst. III. pag. 42; R abenh. p. 297 ; FI. Bat. Tab. 750. 2)

In Herb.: Leiden, $\mathbf{D} z_{\text {. et }} \mathrm{Ml}$ b. ; 's Hage, $\mathrm{O}$ u dem.; Katwijk, W $t \mathrm{w}_{\circ}$; Overveen, Wassenaar, $\mathrm{D}_{z_{\text {}}}$ et $\mathrm{ML}$. Bovendien : Naaldwijk, vo d. Tr. (vo vo et $s_{0}$ )

\section{Trib. Sclerodermacei s}

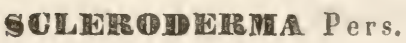

2980. \$. wulgare Fr. In bosschen, op bouw- ch weilanden, enz. (soms aan oude boomironten). Aat. (Fr. Syst. III. p. 46 ; R abenh. p. 295)

In Herb.: Leiden, het Haagsche bosch, Dz. et $\mathrm{II} \mathrm{b}$. Bovendien: Westland, v. d. Tro; Nijmegen (Wychen), A bel. (v. v. s. et ic.)

2851. S. verrucosum P e r s. Op schralen zanderigen boschgrond. Aut. (Fr. Syst. III. p. 49 ; Rabenh. p. 297 ; S. pedunculatum $\mathbf{D} z$. et $I I b$. in Bijdr. V. Tijdschr. Nat. Hist. XI. p. 398)

In Herb.: Leiden, Dz. et $\mathrm{MI}$ b. ; Hilversum, M. D. ; Bij de Bilt en Zeist, het Hagssehe bosch, v. d. S. Lac. (v. v. et s.) Variat:

B. spadiceum Schaeff. Minus, reniforme, saepe sessile。 (Fr. I. I.)

In Herb.: Stadwijk bij Leiden, $\mathbf{D}_{\text {z. }}$ et II $b_{0}$; in hes bosch te Iuiden, v. d. S. Lac. (v. s. et ic.) 


\section{Trib. Cenococcei.}

\section{CENDCDCCEM $\mathrm{Fr}$.}

2882. C. geophillum $\mathrm{Fr}, 0 p$ beschaduwde hei- en zandgronden. Per annum. (Fr. Syst. III. p. 66; Raben h. p. 289)

In Herb.: Voorschoten bij Leiden, $\mathbf{D} z$ et $\mathbf{M}$ b. ; Lisse, 0 udem. (v. vo) Variat.

B. Byssisedum Fr. 1. I. Leiden, met de soort, D z. et M b.

\section{is. Trichodermacei.}

Trib. Trichodermei.

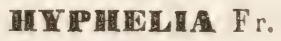

2893. Wl. terrestris $\mathrm{Fr}$. Op vochtige aarde in duisterc bosschen. Vere. Aest. Aut. (Fr. Syst. IlI. p. 213; Rabenh. p. 287)

In Herb.: Leiden, $\mathrm{D}_{\mathrm{z}}$ et $\mathrm{II} \mathrm{b}$. (v. v. et ic.)

2884. 玨. asigrescens $\mathrm{Fr}$. $0 \mathrm{p}$ rottende Esschentronken. Aut. et Vere. (Fr. Syst. III. p. 212; Rabenh.p. 287)

In Herb.: Leiden, $D_{z}$. et $M$ b. (v. v.)

2855. 颉. rosea $\mathrm{Fr} .0$ rottend Populierhout. Vere. (Fr. Syst. III. p. 211; R abenh. p. 288)

In Herb.: Maastricht, Franq.

\section{PILACER F r.}

2886. Pe. Petersii B erk. et Curt. Op roltende schorsen. Hieme.

Stipite breviore candido, capitulo magno, floccis subrectis, sporis minutis sphaericis, vix pallide fuscescentibus. (Rabenh. Fung. Eur. no 268)

In Herb.: Leiden, Dz, et Mb. (v. s.) 


\section{THCHODERTAR Pers.}

3898. T. dubium $\mathrm{Alb}$, et $\mathrm{Sch}$ w. Aan rottende Berkenstammen. Aest. Aut. (F r. Syst. III. pag. 216; R a b e n b. p. 287)

In Herb.: Leiden, $\mathbf{D}$ z. et Mb. (v. v.); Harderwijk, Jungh. in Herb. Dev.

2858. Tr. viride Pers. Aan oude boomtronken en op vuchtig liggend hout. Aut. (Fr. Sysi. III. pag. 215; R abenh. p. 286)

In Herb. : Leiden, D z, et II b.; Zuid-Beveland (Goes), v. d. B.; Maastricht, F r a nq. (v. v. et s。)

2889. T. aeruginosum $\mathrm{Ch}$ ev. Op rottende boomschors. Ant. Vere.

Expansum, sporularum acervis aeruginosum. ( $\mathrm{Ch}$ e $\mathrm{v}$. F l. Par. I. p. 54. Tab. 5. fig. 4. d.)

In Herb.: Maastricht, Franq. (v. s.)

\section{Trib. Aegeritei.}

Aegerita Pers.

285\%. A. candida Pers. Aan rottende boomstammen. Auf. (F r. Syst. III. p. 219; Rabenh. p. 285)

In Herb.: Maastricht, Franq.

\section{My yogteres.}

Trib. Physarei.

\section{HECARPUS Link.}

39DI. T. vernicosus Link, $O_{p}$ stervende grashalmen, doode bladen en takken, in Dennenbosschen. Vere ct autumno, (Rabenh. pag. 284; Diderma vernicosum Pers.; Fr. Syst. III. p. 102)

In Herb.: Leiden (Zuidwijk), B o u r s s e W.; Un. 1850. $\left(v_{0}, v_{0}\right.$, et $\left.s_{0}\right)$ 


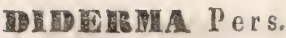

2992. Testaceum Pers. Op Hypnum confertum in holle Wilgen. Aul. (Fr. Syst. IlI. p. 107; Rabenh. p. 282; Desmaz. Pl. Crypt, de Fr. n 706)

In Herb.: Amsterdam, O udem.

2833. D. 6hifforme Summerfo $0 p$ doode bladen en stengels van groene planten. Aut. (Fr. Syst. III. p. 106 ; R a beuh. p. 283)

In Herb.: Zuid-Beveland (Goes), v. d. B. (v. s.)

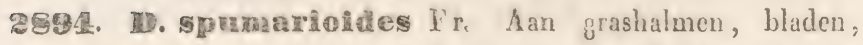
takjes, enz, in bosschen. (Fr. Syst. III. p. 104; Rabenh. p. 283)

In Herb.: Leiden, D zo et $\mathbb{M} \mathbf{b}$. (v. v.)

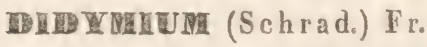

2919. D. chereum (Batsch) Fr. Aan vermold hout in holle Wilgentronken. Aut. (Fr. Syst. III. p. 126 ; R a benh. p. 279)

In Herb.: Zwake, Goes, v. d. B.; Leiden, Dz. et Mi b., 0 ude m. - Bovendien: Westland, v. d.Tr. (v. v.)

2596. He. fratinaceuma Pers. Aan rottend hout, bladen, mossen, enz. Aut. (Fr. Syst. III. p. 119; Ra-. benh. p. 280)

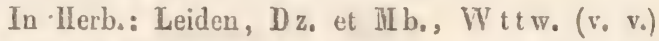

2958. M. Higripes Link. Aan vermolad hout, bladen, enz. - Aut. (Fr. Syst. III.p. 119; R a benh.p. 281) In Herb.: Leiden, $\mathbf{D}$ z et $\mathrm{HI}$ b. (v。 v.)

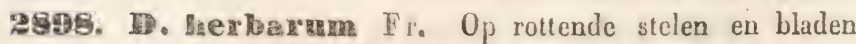
van groene planten. Aest. Aut, (Fr. Syst. III, p. 120 ; Rabenh. p. 281)

In licrb.: Leiden, Dz. et II b.; Amsterdam, v. d.S. Lac. (v. v.) 
2699. praecox $\mathrm{D}$ uhary. $0 p$ rollende bladen van Appel- en andere fruitboomen. Aut. ( $R$ aben h. Fung. Eur. IV. $n^{\circ}, 367$ )

In Herb.: Leiden, $\mathrm{W}_{\mathrm{t}} \mathrm{w}$. ( $\left.\mathrm{v}, \mathrm{s}_{\text {。 }}\right)$

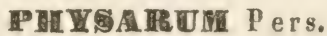

2900. Pr. sinuogum (Weinm.) Fr. Op levende bladen levermossen, Aest. (F r. Syst. III. p. 145; Raben h. p. 273)

In Herb.: Beekbergerwoud, v. d. S. Lac, (v. s.)

2901. Ple. Toryophillam $\mathrm{Fr}$. Aan Hypnum purum en an, dere blad- en levermossen. Aut. (Fr. Syst. III. p. 135; R aben h. p. 275)

In Herbs: Leiden, $\mathrm{D}_{\mathrm{z}}$ e et $\mathrm{Mb}$. ( $\left.\mathrm{v} \nabla_{\text {。 }}\right)$

2902. Ph. nutans Pers. Aan vermolmd hout. Aut, (F r. Syst. III. p. 128; Rabenh. p. 277)

In Herb.: Leiden, $\mathbf{D} z_{\text {。 }}$ et II b. (v。 vo)

2903. Ph. albipes Link. $O_{p}$ vermolmd hout, bladen en rottende planten. Aut. (F r. Syst. III. p. 130; Ph. nutans $\varepsilon$. albipes $R$ abenh. p. 278)

In Herb.: Kampen, R. B o nd. (v. s.) Variat:

B. sessile Dubary. In Herb.: Leiden, $\mathrm{D} z$, et II $\mathrm{b}$. (v. $\nabla$.

\section{CRATERTITrentep.}

2904. C. pedunculatum ' $\mathrm{T}$ rentep. In hosschen op roltende takken en bladen van Eiken en Beuken. Aut. (Fr. Syst. 1II. p, 150; Rabenh. p. 271)

In Ilerb.: Leiden, Dz. et $\mathbf{I I}$ b。; Haarlem, Amsterdam, v. d. S. Lac. (v, vo)

2905. C. minutum Fr. Aan mossen, korsimessen, bla- 
den, takjes, enz. Aut. (Fr. Syst. III. p. 15i; Ra* benh. p. 271)

In Herb.: Leiden, $\mathbf{D}$ z, et $1 \mathrm{Il}$ b. (vov.) Variat:

ค. aureum (Fr. Syst. III. p. 152). Op Hypnum prae. longum. In Herb.: Leiden, W $t \mathrm{tw}$. (v. vo)

2\$06. C. Deucostictum $\mathrm{C}$ hev. $\Lambda$ an cude boomstammen op mossen (Lestiea sericea). Sept. (F r. Syst. III. pag. 152)

Peridio pyriformi erecto candicante, operculo convexo concolore, stipite brevi laevi, sporidiis atris.

Priori perquam afline. Stipes peridio triplo brevior fulvescens; peridium junius plumbeo-lividum dein candicans; margo saepe laceratus. Fr. 1. I.

In Herb.: Zuid-Beveland, v. d. B.; Amsterdam, v. d. S. Lac. (v. s.)

\section{Trib. Stemonitidel.}

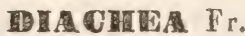

2907. D. elegans $\mathrm{Fr}$. In bosschen en op beschaduwde plaatsen aan dorre stengels en bladen. Aut. (Fr. Syst. 11I. p. 156 ; R abenh. p. 270)

In Herb.: Leiden, Dz. et Ml b. Bovendien: Westland, r. d. Tr. (v. s. et ic.)

\section{STEMIONITES Gled.}

29895. t. fusca $\mathrm{Rolh}$. Aan oude tronken en vermolmde stammen. Aest. Aut. (F r. Syst. III. p. 157; Rabenh. p. 268)

In Herl. : Leiden, D z, ct Ml bo; Zuid-Beveland, vo d. B. Bovendien: Westland (Naaldwijk), v. d. Tr. (v. vo et s.) Variat:

B. Aubuline Fr. 1. 1. Op de bladen van cen Pandanus. In Herb.: Utrecht in den Hort. Acad, , Dz, et $\mathbb{M}$. 
2909. St. Obfusata Fr. Op roltend hout van Esschen. Aut. (Fr. Syst. III. p. 160; R abenh, p, 269)

In Herb.: Leiden, $\mathbf{D}_{z_{0}}$ et $\mathbf{H b}$.

2910. St. typhoides Dec. In hulle Wilyen en an vermolmd hout. Aut. (Fr. Syst. III. p. 158; Rabenh. p. 269)

In Herb.: Zuid-Beveland, $\mathbf{v}, \mathrm{d}, \mathrm{B} .(\mathrm{v}, \mathrm{v}$ )

2911. 5t. ovata Pers. $O_{p}$ vermolmd hout. Aut. (Fr. Syst. III. p. 160; R a be a h. p. 269)

In llerb.: Leiden, Dz. et $M$ b.

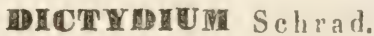

2912. W. umbilleatuma Schrad, In holle Wilgentronken. Sept. Oct. (Fr. Syst. 11l. p. 165; Rabenh. p. 267)

In Herb.: Zuid-Beveland, v. d. B.; Leiden, WV $t$ w. (v. v. et s.)

2913. D. ambigum $\mathrm{Sch}$ rad. $U_{p}$ roltende Dennentronken. Aut. (Fr. Syst. III. p. 165; R a benh. p. 267)

Leiden, $W t t w$. (v. ic.)

2914. ID. Splendens $S \mathrm{chrad}$. Op roltende stammen van Dennen en Beuken, Aest. Aut. (F r. Syst, 11I. p. 166; Rabenh. p. 267)

Leiden, Wuw. (v. ic.)

2915. mierocarpura Sehrad. Op roltend Dennenliout. Aut. (Fr. Syst. III. po 166 ; R a benh. p. 268) Leiden, Wtlw. (vo ic.)

\section{Trib, Trichiaces.}

TUBU⿴囗十⺝木 pers.

2916. T. cylinalrica $\mathrm{Dec}$. Op rollend hout en de selors 
van verschillende boomen. Aest. Aut. (Fro Syst. IIR. p. 195; Rabenh. p. 264)

In llerb.: Leiden, W $\mathrm{ttw}$; Zuid-Beveland (Goes) v. d. B.

2D18. T. Tragiformis Dec. Op rottend hout, in holleWile gen, aan bladen, takjes, mossen, enz. - Aut. (R abenh. p. 264; Licea fragiformis Fr. Syst. III. p. 196)

In Herb.: Nieuwersluis, v.d.S. L a c. (v. s.); Zuid. Beveland, v. d. B.

2918. T. pulvimata Lév. $O p$ de schors van hoomen. Aut.

In Herb.: Leiden, Dz, et Ml b. (v. s.)

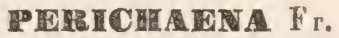

2919. Ptrobllina $\mathrm{Fr}$. Onder de schubben van rottende Pijnkegels, Aut. (Fr. Syst. III. pag. 190; R a ben h. p. 262)

In Herb.: Haarlem, Dzo et Mb. (vo vo et so)

2920, populima Fr. Onder en op de rottende schorg van Populieren. Aut. (Fr. Syst. III. p. 191; R a benh. p. 262)

In Herb.: Leiden, D z. et II b.; Zuid-Beveland (Goes) v. d. B.; Katwijk, W $t \mathbf{t w}$ 。

\section{THE ICHIIA II all.}

2921. Taria Pers, Op run van broeibakken, aan vermolmd hout, enz, - Per totum annum. (Fr. Syst. III. p. 188; Rabenh. p. 259)

In Herb.: Leiden, $\mathrm{D}_{\text {z. et }} \mathbf{M} \mathrm{b}$.; Zuid-Beveland (Goes), v. d. B.; Rotterdam, 0 u dem. (v. v.)

ผ222. T. chrysesperma Dec. Aan vermolmd hout in rottende biadhoopen. Aest. Aut. (Fr. Syst. III, p. 187; Rabenh. p. 259) 


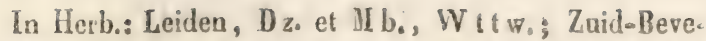
land (Goes), v. d. B. - Bovondien: Westland (Naaldwijk) v. d. Tr, (v. vo et s).

2923. Tr. turoinata With. Aan vermolmd Wilgenhout. Aest. Aut. (Fr. Syst. III. p. 187; Rabenh. p. 260 ; Fl. Bat. Tab. 809. 2)

Naaldwijk, vo d. Tr. (n, vo)

2924. Th. Neesiana Ciord. Aan vermolmd Wilgenhout. Aut. (Cord, Ic. Fung I. p. 23. Tab. VI. กี benh. pag. 260)

In Herb. : Leiden, W $\mathrm{t} t \mathrm{~W}$. ( $\mathrm{v}_{0} \mathrm{~s}_{0}$ )

2925. T. 5eabra Dubary, Op vermolmd hout. Aut. In Herb.: Leiden, Dz。 et M b.; 't Ilaagsehe Boseh , G. B.

2926. T. Trubiformisg Pers. In holle Wilgentronken. Oct. (Fr. Syst. III. p. 183; Rabenh. p. 261). Zuid-Beveland, v. d. B. (v. ic.)

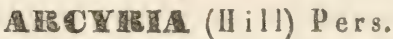

2928. A. nutam Dec. Aan vermolmd Wilgenkout. Aes!. Aut. (Fr. Syst. III. p. 180; Rabeah. p. 257)

In Ilerb.: Zuid-Beveland (Goes), v. d. B. (v. 8.); Leiden, D z. et II b.

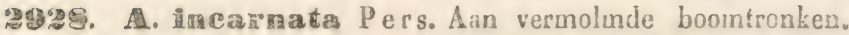
Aug. (Fr. Syst. III. p. 178; Rabenh. p. 258)

In llerb.: Zuic-Beveland, v. d. B. (v. s.)

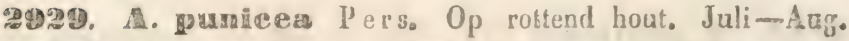
(Fr. Syst. III. paijo 177; Rabenh. p. 258)

In Herb.: Leiden, D zo et II b.; Zuid-Beveland, จ. d. B.; 't Sas van Gend, WVall'a v. (v. s.) 


\section{Trib. Aethalini:}

\section{BUCOEATA Mich.}

2930. H. epidendrum $B \mathrm{axb}$. Aan vermolmde boomtronken, in holle Wilgen, aan oude palen, enz. - Vere. Aut, (Fr. Syst. III. pag, 80 ; R a benh. p. 256; Fl. Bat. Tab. 805. 2)

In Herb.: Leiden, D z. et II b.; Nichtevecht, Utrecht, v. d. S. L a c.; Rentom, B u se; Zuid-Beveland, v. d. B. - Bovendien: Westland (Naaldwijk), จ. d. Tr。 (v. v. et s.)

29931. L. parietimum $\mathrm{Fr}$. Aan vochtig rottend hout en papier (aan oude rochtige bepleisterde muren). Aut, (Fr. Syst. III. pag. 83; Rabenh. pag. 257)

In Herb.: Leiden, $\mathbf{D}$ z, et $M b_{0}$ ( $\left.v_{0}, v_{0}\right)$

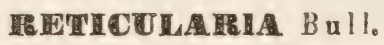

2952. IR. muscorum (A. et S.) Fr. Op mossen, bladen, enz., op vochtige, beschaduwde plaatsen. Aut. (Fr. Syst. III. p. 91 ; $\mathrm{R}$ a be $\mathrm{nh}$. pag. 255)

In Herb.: Leiden, D z. et II b.; Zuid-Beveland, v. d. B. (v. $\left.v_{0}\right)$

2933. Fr. umbrina $\mathrm{F}$ r. Áan oude Dennenpalen, - tronken, eaz. (Fr. Syst. III. p. 87; Rabeñh. p. 255). Aest. Aut.

Westland (Naaldwijk), v. d. Tr. (v. s。 et ic.)

\section{AETHAHUTH L i k}

9934. A. septicum $\mathrm{Fr}_{\mathrm{r}}, \mathrm{O}_{\mathrm{p}}$ de run in broeibakken en oranjeriën. Vere et Aut. (F r. Syst. III. p. 93; R a. benh. p. 253)

In Herb.: Leiden, $\mathbf{D}$ z. et $\mathbf{M b}$. - Bovendien: Westland, v. d. Tr. (v. vo) 


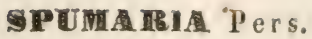

2935. 5. alba Dec. Op weilanden, dan wegen, enz., grashalmen en plantenstengels overtrekkend. Jul.-Sept. (Fr. Syst. III. p. 95; R abenh. p. 253)

In Herb.: Leiden, D z. et II b.; Zuid-Beveland, v. d. B. - Bovendien: Westland, v. d. Tr. (

\section{*** Selerotiaceac.}

\section{Trib. Sclerotiacei.}

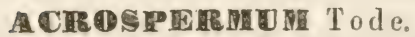

2936. A. compressum Tode. $O p$ doode stengels (vooral op die van Urtica en Carduus). Aut. (Fr. Syst. II. p. 245; Rabenh. p. 244)

In Herb.: Maastricht, Franq. (v. vo et s.)

293\%. 通raminum Lib. Op doode bladen van Grasseu (Poa en Elymus). Aut. (R abenh. p. 244; Lib. PI. cr. Arduenn. $\mathrm{n}^{\circ}$ 33.)

In Herb.: Zuid-Beveland (Goes), จ. d. B. (v. s.)

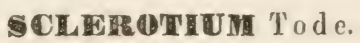

2939. S. Clavus Fr. $\Lambda$ an de aren van verschillende Gramineeën. Aest. (Fr. Syst. II. p. 269; R a b e $n$ h. p. 238). Variat.

a. Secales Rabenh. I. I. In Ilerb.: Geertruydenbery, Verkouteren; A peldoorn, v. d. S. L ac.; Nijmeggen, Abel. (v. so)

f. Tritici W all r. In Herb.: Leiden, Dz, et Mb. (r. r.) Maastricht, Franq.

y. Lolii Wallr. In Herb.: Leiden, Dz. et II b.; $\Lambda$ mo sterdam, O udem. (v. จ. et s.) 
ס. Agropyri West. (In Herb.) Op Agropymm repens.

In Herb.: Leiden, $\mathbf{D}$ z. et $\mathbf{I I}$ b. (vo v. et s.)

ع. Arundinis West. (In Herb.) $0 \mathrm{p}$ Arundo Phragmites.

In Herb.: Zuid-Beveland (Goes), vo d. B.

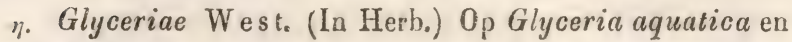
fluitans.

In Herb. : Leiden, D z. et II b. ; Leesten, A b e l. (vo s.)

2989. ฐ. Pustula Dec. $0 p$ doode Eikenbladen. Aut. (F r. Syst. II. pag: 260; R a b e $\mathrm{nh}$. p. 239)

In Herb.: Haarlem, 0 u d e m. (v. s.). Variat :

ค. epicaula West. $O_{p}$ doode Agrdappelstengels.

In Herb.: Westland (Naaldwijk), vo d. Tr.

2940. 5. albidum Rob. $O p$ doode Wijngaardranken. Vere et Aut.

Erumpens, minutum, rotundatum, dein oblongum, confluens, convexum, albidum, sublaeve, siccum depressum, intus concolor. (Rob. in Des uaz. Pl. Crypt. de Fr. Nouv. Edit. $\mathrm{n}^{\circ}$ 567)

In Herb.: Zuid-Beveland, v. d. B. (

2941. 5. Gurum Pers. $O_{p}$ doode stengels van groene planten. Aut. (Fr. Syst. II. p. 259; Rabenh. p. 239) Variat:

a. Op Solanum tuberosum. In Herb.: Leiden, D $z_{0}$ et II b.; Maastricht, Fran q.

b. Op Anethum Foeniculum. In Ilerb。: Goes; v. A. B.; Groningen, Strat.

c. Op Heracleum Sphondylium. In heri.: Leiden, Oud e m.; Utrecht, v. d. S. L a c,

d. Op Convolvulus Sepium. In Herb,: Amsterdan, $\forall$. d. S. $_{\text {. }}$ $\mathbf{L}$ a c. ; Ubbergen bij Nijmegen, $\boldsymbol{A}$ bel.

c. Op Polygonum avicuiare. In Herb.: Zuid-leveland, v. d. B.; Amsterdam, v. d. S. L a c.

7. $\mathrm{O}_{\mathrm{p}}$ Aster Tripolium, In Herb,: Amsterdau ve d. S. Lac. (vo vo et s.) 
2942. \$. Heiodermon $\mathrm{Rob}, \mathrm{Op}_{\mathrm{p}}$ doude stengeis var Polygonsm Persicaria, dikwijls in gezelschap van S. durum. Vere.

Adnatum, laxe gregarium, minulum, rolundatum ovatumque, confluens, nigrum, laeve, nitidulum, intus album: (Rob. in Desmaz. Pl. Crypt. de $F_{\text {r. }}$ Nouv. Ed. $\mathbf{n}^{\circ}$ 568)

In Ilerb.: Leiden, in den Horius academ., Ju ng h.

2943. Tiliacearum $W$ est. $0 p$ de zaadhuisjes en stengels der Liliacceën. Vere.

Sparsum, adnatum, parvum, orale, pallide fuscam, laeve, demum nigrum, rugosum, intus album (S. Tulipae Lib. Pl. Crypt. Arduenn. $\mathrm{n}^{0}$ 36). Variat:

B. Scillae West. In Herb. Cr. Belge. Op Scilla nutans en Allium vineale.

In Ilerb.: Dongen (Noord Braband). (v. so)

r. Tulipae West. Op wilde en gekweekte Tulpen。 Mlaastricht, Franq. (n. v.)

2941. \$. compactum Dec. $O_{p}$ den bloembodem, de vrachten en de zaden van de Cucurbitaceeën en de Helianthideeën. Vere. (Fr. Syst. II. p. 258; R a b e n h. p. 239). Variat :

B. Helianthi $\mathrm{R}$ abenh. I. I. In lierb.: Zuid-Beveland (Goes), v. d. B. - Bovendien: Maastricht, Fran q.

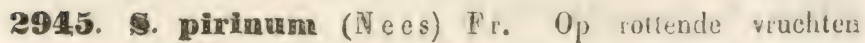
(kweeën, appelen, enz.) Vere. (Fr. Syst. II. p. 258; Rabenh. p. 240)

In Herb.: Utrecht, W $t \mathrm{t}$ w. (v. $\left.s_{0}\right)$

2946. \$. varium Pers. $0 p$ aardappelen, gele wortelem en rapen, in kuilen en kelders. Vere. (Fr. Syst, $\mathbf{I I}_{\text {。 }}$ p. 257; Rabenh. p. 240) Haastricht, Fran q. (u. v.)

2948. S. muscorum Der'sp Aan de wortels van ver- 
schillende mossoorten, op vochtige en beschaduwde plaatsen. Aut. Vere. (Fr. Syst. II. p. 252; R a benh. p. 241)

2945. Torillosum Lib.? $O_{p}$ de run in broeibaklen en oranjeriën. Aut. Vere:

Tectum, magnum, difforme, durum, primo laeve album, demum corrugatum umbrinum, intus stuppeofibrosum, fibrillis ramosis repentibus candidis instruclum. (Lib. Pl. Crypt. Ard, no 139)

In Herb.: Zuid-Beveland (Goes), v. d, B.

2949. Semen Tode. $0 p$ en in doode stengels van Solanum tuberosum en Arctium Lappa, op rottende Populierbladen en afgevallen zaadhuisjes van Fraxinus. Vere. (Fr. Syst. II. p. 249; R abenh. p. 243)

In Herb.: Leiden, $\mathrm{D}_{\mathrm{z}}$, et II b.; Haarlemmerhout, De H a a ; Amsterdam, v. d. S. Lac: - Bovendien: Miastricht, Franq; Westland, v. d. Tr. (v. v. et s.)

2950. ๑. Pezmeforme Schum. $O_{p}$ doode Populierbladen. Aut. Vere. (Fr. Syst. II. p. 248; R a ben h. p. 243)

In Herbs: Utrecht, v. d. S. Lac. (r. s.)

2951. S. scutellatum $A / b$, et $S c h w . ~ O p$ rottende bladen. Vere. (Fr. Syst. II. p. 248; R a benh. p. 243) In Ilerb.: Amsterdam, v. d. S. Lac. (v. s.)

2952. ๑. complanatram 'T'ode. Op rottende Populierbladen. Aut. Vere. (Fr. Syst. II. p. 248; Rabenh. p. 243)

In Herb.: Amsterdam, v. d. S. Lac.; Utrecht, v. d. S. Lac. - Bovendien: Mastricht, Franq.

2953. S. crastuliforme Rob. Tusschen de vezels der bladstelen van verschillende boomen. Vere.

Tectum, minutum, ovatum vel ellipticum, applanatum, siccum, rugulosum, primo pallidum, demum brunneo-castaneum; infus album. (Rob. in Desmaz. 
15 notice sur les pl. erypt. de Fr. in Ann. des Sc. Nat. 3e Série. T. IX. p. 346 ; Desmaz. Pl. Crypt. de Fr. $n^{\circ}$ 2046). Variat:

B. Iridis W est. 8e Not. Cr. inéd. Belg. $n^{\circ} 23$. Tusschen de vezels van rottende Irisbladen. In Herb.: Amsterdam, v. d. S. L a c. (v. s.)

2954. S. medullosum $\mathrm{Rob}$. Op doode Olmtakken. Vere,

Erumpens, subtectum, minutum, ovatam vel ellipticum, primo convexum pallide-griseum, basi tenui byssinae insidens, demum depressum nigrescens, intus medullosum sordide albido-ochroleucum. ( $\mathrm{R}$ o b. in Desmaz. 22e Notice sur les pl, crypt. de Fr. in Ann. des Sc. Nat. 3e Série. T. XX, $\mathrm{n}^{0}$ 33. - Desmaz. PI. Crypt. de Fr. Nouv. Série. no 160)

In Herb.: Amsterdam, v. d. S. La c. (v. s.)

2955. 5. variegatum $\mathrm{W}$ allr.? In de stengels van groote groene planten. Vere.

Pseudo-stromate ovato-subgloboso adnato, dein elongato ruguloso ex albo-nigroque variegato, intus corneoalbo. (W allr. Comp. Fl. Germ. IV. p. 139).

In Herb.: Holland, Kros (v. s.)

2956. S. Sinapispermum West. $0 p$ vochtige ran in broeibakken. Aut. Vere.

Péridium sphérique, d'un demi a un millimètre de diamètre; à l'état frais jaunâtre, plus ou moins orangé, lisse et adhérent par un point; à l'état sec libre, d'un rouge hrun et légèrement chagriné à la surface. Chair blanche et cornée. West. 8e Not. Crypt. inéd. de la Fl. Belg, $\mathrm{n}^{\circ} 24$, pag. 8.

In Herb.: Leiden, $\mathbf{D z}$. et $\mathbb{M}$ b. (vo vo et s.)

\section{DIDUANESDIRA $\mathrm{C}$ ast.}

Spores? ou Sporanges? cylindriques, parallèles, souyent de consistance cornée, arrondies aux deux extré. 
mités ou accompagnés à la base par une ou deux utricules arrondies. (Castagne Cat. Pl. de Mars. p. 206)

295\%. M. Fuphorbiae Cast. Op treurende bladen van sommige soorten van Euphorbia. Aut. (Sclerotium herbarum $\beta_{0}$. Eáphorbiae Kze., Fr. Syst. II. p. 263)

In Herb.: Leiden, Overveen, $\mathrm{D}_{\text {。 }}$ et $\mathbf{I I} \mathrm{b}$.; ZuidBeveland, v.d. B.; Ewijk, A bel. - Bovendien: llaastricht, Franq. (sub nom. Rhytisma Euphorbiae).

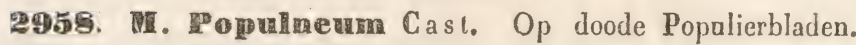
Aut. (Sclerotium Populneum Pers, Fr. Syst. II. p. 262; Perisporium Rabenh. p, 228)

In Herb.: Leiden, D z. et M b., Oudem.; Utrecht, W ttw. - Bovendien: Maastricht, Franq. (v. vo et s.)

2959. W. Salleinum Desmaz, Op treurende Wilgenbladen. Aut. (Sclerotium Salicinum Dec., Fr. Syst. II. p. 262 ; Perisporium Rabenh. p. 228) In Herb.: Leiden, $\mathbf{D} z_{\text {o }}$ et $\mathbf{M}$ b. (v。 vo et 's.)

\section{Trib. Perisporel.}

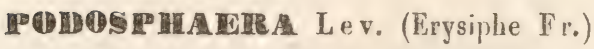

Mycelium effusum arachnoideum plerumque evanidum. Conceptacula sphaerica sporangio unico, subgroboso, octosporo farcta; sporac ovatae. Appendiculae parcae (3-8), dichotomae, fuscae, apice turgidae, hyalinae. (Lév. Org. et Disp. méth. du G. Erysiphe in Ann, des Sc. Nat. 3e Sér. Tom. XV. p. 109)

2960. P. clandestina A. Crataegi Lev. I. I. Op de levende bladen van Crataegus Oxyacantha. Aest. (Erysiphe Oxyacanthae Dec., Rabenh. p. 237; Fr. Syst. III. p. 238).

In llerb.: Zuid-Beveland, v. d. B. (v. v. et s.) 


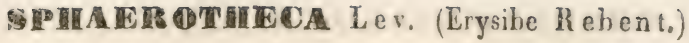

Mycelium arachnoideum floccosum effusum plerumque persistens. Conceptacula glohosa, sporangio unico resiculoso octosporo fareta; sporae ovatae. Appendiculae numerosae floccosac cum mycelio intertextae. (Lev. I. I.)

2961. 5. pannosa $L$ e v. $0 p$ de takken, bladen en bloemkelken der Rozen. Aest. (Erysibe pannosa Link., Fr. Syst. IIl. p. 236; Rabenh. p. 230)

In Herb.: Leiden, Dz. et II b.; Weart bij Nijmegen, A bel.; Zuid-Beveland (Goes), v. d. B.; Rotter* dam, 0 udem.

2962. Castagnei Lev. Op levende bladen van verschillende groene planten. Aest. Variat:

a. Compositarum Lev. Op de bladen van Senecio vulgaris. (Erysibe communis d. Compositarum $\mathrm{R}$ abenh. p. 233; F r. Syst. III. p. 247). In Herb.: Amsterdam, v. d. S, L a c. (v. S.)

b. Veronicarum Lev. I. I. Op de bladen van Veronica longifolia. (Erysibe fuliginea Link.; Rabenh. p. 230 ; Fr. Syst. III. p. 238)

In Herb.: Maastricht. Franq. (v. s.)

c. Plantaginis Lev. 1. 1. Op Plantago lanceolata (Erysibe lamprocarpa $c$. plantaginis Link (partim); R abeuh. p. 232)

In Herb.: Naastricht, Franq. (v. s.)

d. Humuli Lev. 1. I. Op de bladen der Hop (Erysibe macularis a Humuli $\mathrm{Rabenh}$. p. 231; Fr. Syst. III. p. 237)

In Herb. : Leiden, $\mathrm{Dz}$. et $\mathrm{Ml}$ b. ; 's Gravenhage, $\mathrm{O}$ ud e m.; Zuid-Beveland (Goes), v. d. B.; Zwijndrecht , v. d. S. Lac. - Bovendien: Maastricht, Franq. (v, v, et s.) 


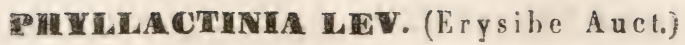

Blycelium amphigenum; conceptacula hemisphaerica demum depressa, receptaculo membranaceo-granuloso persistenti vel evanido insidentia, sporangiis 8 et ultra in pedicellum protractis 2- vel 4-sporis farcta; sporae ovatae. Appendiculae $8-16$ rectae, rigidae, aciculatae, demum retroflexae. (L e v. 1. I.)

9263. - guttata Lev. Op* de levende bladen van ver. schillende kruiden en boomen. Aest. (Erysibe spec. R abenh.). Variat:

a. Aceris West. $O_{p}$ Acer campestris. (Erysiphe marissalii. West. Herb. Cr. Belg. $n^{0} 551$; West. 2e Not. s. qq. Crypt. nouv. fl. Belge in Bull. de l'Acad. Roy. des Sc. de Belg. No. 120. Pl. 1. Fig 4. n. o. p. q.)

In Herb.: Leiden, Dz. et II b. (v. v.)

b. Fraxini L e r. Op Fraxinus excelsior. (Erysibelenticularis a. Fraxini. Rabenh; Fr. Syst. III. p. 246; Rabenh. pag. 234)

In Herb,: Maastricht, Franq. (v. v. et s.)

c. Betulae Lev. Op de bladen van Betula alba. (Erysibe guttata $b$. Betulae Rabenh. p. 234; Fr. Syst。 III. p. 245)

In Herb.: Haastricht, Franq. ( v $^{\left.s_{0}\right)}$

d. Fagi Lev. Op de bladen van Beukenboomen. (Erysibe lenticularis b. Fagi $R$ abenh. p. 234; Fr. Syst. III. p. 246).

In Herb.: Renkom Buse. - Bovendien: Maastricht, Franq.

e. Coryli Le v. $0_{p}$ de bladen des Hazelaars. (Erysibe guttata $a$. Coryli, Link, Rabenh. p. 234; Fr. Syst. III. p. 245)

In Herb.: Leiden, Dz. et Mb.; Zutphen, W $t \mathrm{t}$.; Maastricht, Franq. (v. vo) 
URCHTUR Lev. (Erysibe spec. Rabenh.)

Mycelium epi- vel hypophyllum, floccosum, submembranaceum, evanidum vel persistens. Conceptacula globosa sporangiis 8-16 subpyriformibus, 2-4 sporis ovatis repleta. Appendiculae rigidae, simplices, bifidae vel raro dichotomae apice uncinatae, radiato-patenies, demum sursum flexae. (Lev. 1. 1.)

2961. U. adumea Lev. Op levende bladen van verschillende kraiden en hoomen. (Erysibe adunca $R$ abe $\mathrm{nh}$.) Variat:

n. Artemisiae Lev. Op Artemisia vulgaris. Aest. (Erysibe depressa b. Artemisiae Link.; Rabenh. p. 232; Fr. Syst. III. p. 240)

Haastricht, Fran q. (v. v.)

b. Salicis Lev. $O_{p}$ de bladen van verschillende Wilgen. (Erysibe adunca $a$. Amentacearum bb. Salicum Rabenh. p. 236; Fr. Syst. III. p. 245)

Maastricht Franq. (v. vo)

c. Populi L e v. Op de hladen van verschillende Populieren. (Er. adurica a. Amentacearum. aa. Populorum $\mathrm{R}$ abenh. p. 236.)

In Hlerh.: Maastricht, Franq. (v. v.)

2965. W. Wallrothiif $L \mathrm{ev}, 0_{p}$ de bladen van verschillende: Pruimen, vooral van Pr. spinosa. Aest. (Erysibe adunca c. Rosacearum Wallr,; Rabenh. p. 236; Fr. Syst. III. 2\$5)

Maastricht, Franq.

2966. U. Hornis $L e v . ~ O p$ de bladen van Phylleria ('n verschillende soorten van Acer. Aest. Aut. Variat:

a. Aceris Lev. Op de bladen van Acer campestris, Pseudoplatanus, enz. (Erysibe bicornis (Acerum) Rahenh. p. 235; Fr. Syst. III. p. 244)

In Herb.: Leiden, D z. et II b. ; Rolterdam, 0 a d e m.; Kampen, Bond.; Zuid-Beveland (Goes), v. d. B. Bovendien: Maastricht, Fran . . (v. v.) 
CALDCLATIA Lev. (Erysibe spec. A uet.)

Mycelium arachnoideum, subcontextum, evanidum vel persistens. Conceptacula sporangiis $4-8$ ovatis rostratis 4-8 sporis repleta. Appendiculae rectae dichotomae, ramulis apice turgidis vel filiformibus. (Lev. l. 1.)

2968. C. Ehrenbergii Lev. $O_{p}$ de bladen van Lonicera tatarica. Aest.

IIycelio epiphyllo subcontexto orbiculari persistente. Coneeptaculis gregaricis globosis minutis. Sporangiis 4 ovato-rostratis 8 sporis. Appendiculis circiter 20 conceptaculum aequantibus. (L e v. I. I.)

In Herb.: Leiden, $\mathrm{D} z$, et $\mathrm{II}$ b.

2968. C. penichlata $L e v .0 p$ de bladen van verschillende boomen. Aest. et Aut. (Erysibe spec. Rabenh.) Variat:

a. Viburni Lev. Op de bladen van Viburnum Opulus (Erysibe penicillata $\beta$. Caprifoliacearum Rabenh. p. 236 ; Fr. Syst. III. p. 242).

In Ilerb.: Wassenaar, Dz. et II b. ; Amsterdam, $\mathrm{O}$ udem. (v. s.)

2969. C. Bubyi Lev. $O_{p}$ de bladen van Lonicera $X y$ losteum, Caprifolium, enz. (Er. penicillata $\beta$. Caprifoliacearum pro parte. Rabenh. p. 236).

In Herb.: Zuid-Beveland (Goes), v. d. B. (v. s.)

2980. C. holosericea Lev. $O p$ de bladen van $A s$ tragalus glycyphyllos. Aest. (Erysibe holosericea Astragali, Rabenh. p. 231 ; Fr. Syst. III. p. 240) In Herb.: Ubbergen en Weurt bij Nijmegen, A bel. (v. s.)

2981. C. Berberidis $L$ ev. $0 p$ de levende bladen van Berberis vulgaris. Aest. Aut. (Erysibe penicillata c. Berberidis Link; Rabenh, p. 236; Fr. Syst. III. p. 244) 
In Ilerb.: Leiden, $\mathbf{D}$ z. et $\mathrm{Ml}$ b. ; Rotterdam, O u dem; Ilees bij Nijmegen, A bel.; Zuid-Beveland (Goes), v. d. B. - Bovendien: Maastricht, Franq. (v. vo et 8.)

2982. C. Grossulariae Lev. $O p$ de bladen van Ribes Grossularia. Aest. Aut. (Erysibe penicillata d. Grossulariae Link.; Rabenh. p. 236)

In IIerb.: Amsterdam, v. d. S. L a c.; Zuid-Beveland (Goes), v. d. B. - Bovendien: Maastricht, Fran q. (v. 8.)

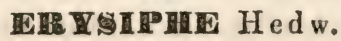

Mycelium arachnoideum saepe contextum, fugax vel persistens. Conceptacula globosa aut hemisphaerica, sporangiis 8-24 polysporis repleta. Appendiculae floccosae nunc simplices nunc vage ramosae cum mycelio intertextae, interdum liberatae (L ev. emend. I. I.)

2983. Hamprocarpa Lev. $O_{p}$ de bladen van verschillende kruiden. Aest. Variat:

a. Cichoraccarum $\mathrm{I}_{4} \mathrm{ev}, \mathrm{O}_{\mathrm{p}}$ de bladen van Scorzonera hispanica en Hieracium sabaudum. (Erysibehorridula b. Cichoracearum Wallr.; Rabenh.p. 235; Fr. Syst. III p. 241)

In Ilerb.: Leiden, Dz. et M b.; Weurt bij Nijmegen, A bel.; Zuid-Beveland (Goes), v. d. B.; Maastricht, Franq. (v. vo et s.)

b. Labiatarum. Le v. $O p$ de bladen van Lamium purpureum en album, Stachys palustris, Ballota nigra, enz. (Erysibe lamprocarpa a. Labiatarum Rabenh. p. 232)

In llerb.: Zuid-Beveland, v. d. B.; Leiden, Dz, et II b.; Kampen, B ond.; Rotterdam, $\mathrm{O}$ udem.

c. Plantaginis Lov. $O p$ de bladen van Plantago major en maritima (Erysibe lamprocarpa c. Plantaginis Link.; Rahenh. p. 232; Fr. Syst. III. p. 239) 
In Herb.: Leiden, 0 udem.; Dordrecht, Amsterdam, v. d. S. Lac.; Maastricht, Franq. (v. vo et s.)

2981. E. graminis Dec. $0 p$ de bladen van Apera Spica venti, Agrostis alba, enz., in de koornvelden. Aest. (Erysibe communis a. Graminearum Link.; Rabenh. p. 232)

In Ilerb.: Rijnsburg, Leiden, $\mathrm{O}$ ude m.; Amsterdam, v. d. S. L a c.

29 schillende kruiden. Aest. Aut. Variat:

a. Cruciferarum Lev. Op Alyssum calycinum (Erysibe communis $p$. Craciferarum $\mathrm{R}$ abe $\mathbf{n h}$. p. 233; $\mathrm{F}_{\text {r }}$ Syst. III. p. 242)

In Hérb.: Weurt bij Nijmegen, A b el. (v. v.)

b. Leguminosarum Lev. $\mathrm{Op}_{\mathrm{p}}$ de bladen van Pisum sativum, Melilotus alba, als ook van Lupinus, $V i$ cia, Trifolium, enz. (Erysibe communis m. Leguminosarum Link; Rabenh. p. 233; Fr. Syst. III. p. 240)

In Herb. : Leiden, Dz. et $\mathrm{Ml}$ b; $0 \mathrm{u}$ de m.; Heerjansdam, v. d. S. L a c.; Rotterdam, O u de m.; Nijmegen, A bel.; Kampen, Bond.; Zuid-Beveland, v. d. B.; Amsterdam, v. d. S. La c.; Maastricht, Franq. (v. v.)

c. Ulmariae Lev. Op de bladen en bladstelen van Spiraea Ulmaria. Aest. Aut. (Erysibe horridula $c$. Spiraeacearum $R$ abenh.?. p. 235)

In Herb.: Zuid-Beveland, Zwake, v. d. B. (v. s.)

d. Umbelliferarnm Lev. Op de bladen van Anthriscus sylvestris, Heracleum Spondylium en andere Uimbelliferae. (Erysibe communis $l$. Umbelliferaram Link; Rabenh. p. 233; Fr. Syst. III p. 242)

In Herh.: Zuid-Beveland, v. d. B.; Maastricht, Franq.; Rotterdam, Oudem. (v. v. et $s_{\text {. }}$ )

c. Urticearum Lev. Op de bladen der Netels, en bijzonder op die van Urtica dioica en urens. Aest. Aut. 
(Erysibe communis b. Urticearum Rabeuti: p. 2333) In Ilerb.: Amsterdam, v. d, S. Lac.

2986. E. Mlontagnei Lev. $O_{p}$ de bladen van kruiden, zoo als Lappa, Cirsium, enz. Aest. Aut. Variat:

a. Bardanae Lev. Op de bladen van Lappa major en tomentosa. (Erysibe depressa a. Bardanae Link: Rabenh. p. 232; Fr. Syst. III. 242.)

In Herb.: Leiden, $\mathbf{D}$ z. et $\mathrm{Ml}$ b.; Dordrecht, $\mathbf{A m}$ sterdam, v. d. S. Lac.; Zuid-Beveland, \& d. B.; Haastricht, Franq. (

2987. tortilis Fr. $O p$ de bladen van Cornus albu en sanguinea. (Erysibe tortilis Corni Link; $\mathrm{Ra}$. benh. p. 231; Fr. Syst. III p. 243)

In Herb.: Rotterdain, in de nicuwe plantaadje, 0 adem.; Maastricht, Franq. (จ. vo et 8.)

2985. Worridula Lev. $0 p$ de bladen van Symphytum officinale, Echium vulgare, Myosotis intermedia, enz. Aest. Aut. (Erysibe horridula $a$. Asperifoliarum R a benh. p. 235 ; Fr. Syst. III. p. 239)

In Herb.: Leiden, Dz. et $\mathrm{Ml}$ bo; O udem.; Amsterdam, v. d. S. La c.; Zaid-Beveland, v, d. B.; Ewijk, A bel. (v. s.)

2989. C. communis $\mathrm{Fr} .0_{p}$ de bladen van verschillende kruiden. Aest. Aut, Variat:

a. Ranunculacearum Duby. $\mathrm{Op}$ de stengels, blatstelen en de bladen van Aquilegia vulgaris, Ranunculus acris, Philonotis, enz. (Erysibe communis $r$. Ranunculacearum Link; R abenh. 1. 233; F r. Syst. III. p. 240)

In Herb.: Leiden, Dz. et HI b.; Zuid-Beveland, v. d. B.; Maastricht, Frauq.; Beuningen bij Rijuegren, $\mathrm{A}$ bel.

b. Polygonearum Eeva Op de bladen van Polygoniai. 
aviculare (Erysibe communis $r$. Polygonearam Link; Rabenh. p. 233; Fr. Syst. p. 240)

In Herb.: Amsterdam, vo d. S. L a c.; St. Anne bij Nijmegen, A b e l. (v. v.)

c. Solanacearum West. $0_{p}$ de bladen van Verbascum Thapsus. Aest. (Erysibe communis $k$. Solanacearum Rabenh. p. 233; Fr. Syst. III. p. 242)

In Herb.: Leiden, $\mathbf{D} z$. et $\mathrm{M}$ b. (v. 8.)

d. Rubiacearum West. $O_{p}$ de bladen van Galium Aparine. Aest. (Erysibe communis do Rubiacearum Rabenh. p. 233)

In Herb.: Leiden, $\mathbf{D}$ z. et $\mathrm{M}$ b.; 0 udem.

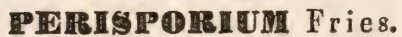

2980. P. vulgare $\mathrm{C}_{0} \mathrm{rd}$. $0 p$ de bladen van Phragmites communis. Aut. Vere. (Fr. Syst. III. p. 250; Rabenh. p. 228; Perisporium arundinis Desmaz. Pl. Crypt. de Fr. $n^{\circ}$. 329)

In Herb.: Zuid-Beveland, v. d. B.

2981. L. Hrassicae $\mathrm{Li}$ ib, $0 \mathrm{p}$ rottende stronken van roode en groene kool. Vere. (Lib. Pl. Crypt. Ard. n . 280; Sclerotium, Coccocystis, Brassicae Pers.; Rabenh. p. 239; Fr. Syst. III. p. 259)

In Herb.: Leiden, W $\mathrm{t} t \mathrm{w}$. - Bovendien: Leiden, Dz. et II b.; Zuid-Beveland, v. d. B.; Maastricht, Franq.

\section{CONHOSPORIUPR Lin.}

2982. C, circinans Fr. Op de bladscheeden van Phragmites communis. Aut. (Fr. Syst. III. p. 257; Desmaz. Pl. Crypt. de Fr. no. 330; Sphaeria circinans Rabenh. p. 174)

In Herb.: Leiden, W ttw.; Amsterdam, v. d. S. L a c.; Zuid-Beveland (Goes), v. d. B. (v。 v. et s.) 
IICIR diticta Desmaz. (Perisporii spec. Fr.)

Perithecium? minutum scutiforme carbonaceum celIulosum superficiale-adnatum, intus nucleo? carnosocelluloso albo ubique fructificante. (Desmaz. Pl. Crypt. de Fr. Nouv. Edit. $\mathrm{n}^{\circ}$. 674)

2953. FI. vagans Des maz. $0_{p}$ de stengels en bladen van verschillende kruiden en heesters (Perisporium Speireum Fr. Syst. III. p. 230) Aut. Vere. Variat:

a. Buxi West. $0 p$ de bladen van den Palmboom. (West. 7e not. Crypt. Belg. $\mathrm{n}^{\circ}, 17 d$. in Bull. acad. roy. Sc. de Belg. 2e Série T. XI)

In Herb.: Zuid-Beveland (Goes), v, d. B. (v, v.)

\section{Trib. piosporel.} CHAETONIU⿴囗十⺝ $\mathrm{K} z$ et $\mathrm{Sch}$.

2984. C. Clatum $\mathrm{Kz}$ e et $\mathrm{S} \mathrm{ch}$. Op rottende grashalmen, riet en bladen. Per totum annum. (Fr. Syst. Ill. p. 254; Rabenh. p. 227)

In Herb.: Leiden, Dz. et II b.; Wilhelminapolder, Zuid-Beveland, v. d. B. (v. s.)

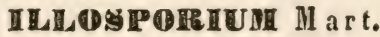

2985. I. carneum $\mathrm{Fr} . \mathrm{Op}$ het thallus van Peltigerasoorten. Aut. Vere. (Fr. Syst. III. po 259; Rabenh. pag. 225).

2956. 耳. coccineum $\mathrm{Fr}$. Op het thallus ran korstmossen. Aut. Vere. (Fr. Syst. III. p. 259; Rabeah. p. 226) Manstricht, Franq. (n. v.) 


\title{
Ord. Pyrenomycetes.
}

\author{
* Ipha eriacei
}

\author{
Trib. Iphaerini.
}

\section{CORDYCERS Fr.}

298\%. C. Dphloglossoldes Fr. In vochtige bosschen, gewoonlijk parasitisch op Elaphomyces muricatus. Aut。 (Fr. Syst. II. p. 324; Rabenh. p. 224)

In Herb.: Renkom, Buse.

2985. C. militaris $\mathrm{Fr} . \quad \mathrm{p}$ doode insectenlarven in bos schen. Aut. Vere. (Fr. Syst. II. pag. 323; R a benh. pag. 224)

Westland (Naaldwijk), v. d. Tr. (v. icon.)

\section{FOHONHA Fries.}

2989. P. pumctata Fr. Op paarden- en ezelsmest in zandige streken. Aut. (Sphaeria Fr. Syst. II. p. 330; Rabenh. p. 223)

In Herb.: Harderwijk, Jungh.; Sloten, v. d. S. Lac. - Bovendien: Westland (Naaldwijk), v. d. Tr. (v. 8, et ic.)

XYLAFIA Schran。

20990. X. Corauta Schrank。 op rottende Elzentronken. 
Aut. Vere. (llyposylon vulgare habenh. p. 222; Sphaeria Hypoxylon Fr. Syst. II. p. 327; D z, et H b. in Bijdr: 1. pag. 19).

In Herb.: Kampen, B ond.; Leiden, O udem.; Wit w.; Ubbergen, A bel.; Sterrebosch (Groningen), Strat.; Swijudrecht, v. d. S. Lac. - Bovendien: Westland (Naaldwijk), vo d. Tr.; Mlaastricht, Franq. (v. v. et s.).

2991. X. Cupressiformis $\mathbf{K} x$. $O_{p}$ rottende boomtronken. Aut. Vere. (Sphaeria Hypoxylon $\beta$. Cupressiformis Fr. Syst. II. pag. 328 ; D z. et $11 \mathrm{~b}$. Bijdr.)

In Herb.: Leiden, Dz. et MI b.; W $九$ w.; Haarlemmerhout, v. d. S. Lac.; Zuid-Beveland, v. d. B. (v. v. et s.) Variat:

a. pedata $\mathrm{W}$ est. in llerb. $\mathrm{O}_{\mathrm{p}}$ rottende boomtronken. (Fr. 1. 1.)

In Herb.: Leiden, D z. et MI b.; Dordrecht, v. d. S. Lac.

2992. X. polymorpha Grev. Aan afgehouwen rottende boomtronken. Hieme. (Sphaeria Pers.; Fr. Syst. II. p. 328; Hypoxylon Rabenh. pag. 222)

In Herb.: Dordrecht, v. d. S. La c.; Sterrebosch bij Groningen, Strat. - Bovendien: Westland (Naaldwijk), v. d. Tr.; Maastricht, Franq. (v. v. et s.)

2903. X. digitata Rabenh. Op doode Elzentronken. Aut. Vere. (Sphaeria Fr. Syst. II. 326; Hypoxylon R a benh. Deutschl. Krypt. Flora I. p. 223; Rabenh. Herb. viv. myc. 2e Ed. $\mathrm{n}^{\circ}$. 46)

In Herb.: Leiden, W $t \mathrm{tw}$. (v. s.)

2994. X. carpophilla West. $0 p$ de napjes der Beuken. Ilieme. Vere. (West. Herb. Crypt. Belg. no. 114; Sphaeria. Fr. Syst. II, p. 328; llypoxylon Rabenh. pag. 223)

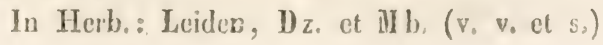


2995. X. coronata West. Op rottende tronken en wortels van boomen. Aut. Vere. (Sph. Guepini Moug.? in herb. Franq.)

Tige allongée, mince, stipitiforme, tortueuse, inégale enbosselée, longue de 35 a 40 mill., s'évasant vers le haut en une tête conique de 4 mill. de longueur, surmontée de 3,4 ou 5 appendices digitiformes d'environ 2 mill. de longueur. Perithèces rares, petits, immergés, placés vers le haut de la tête et devenant saillants. Ostioles papilliformes. Thèques cylindriques, octospores, unisériées, longues de $1 / 10^{\mathrm{e}}$ de mill. et entourées de quelques paraphyses rares, hyalines et simples. Sporidies brunes, ovales, translucides, mesurant $3 / 200^{\text {es }}$ de mill. dans le grand diamètre sur $1 / 100^{e}$ de mill. dans le petit. (W est. $8^{\mathrm{e}}$ not. Crypt. inéd. Belg. pag. 5. no 2. fiğ. 1 a, b, c, d.)

In Herb.: Haastricht. Franq. (v. 8.)

\section{SPPIALUIA II all.}

\section{A. Compositae.}

Sect. 1. Periphaericae. Compositac. Perithecia subdivergentia, in ambitu stromatis collocata, ostiolo aequali, collo destituta.

* Stroma sessile, convexum, subhemisphaericum, immarginatum, peritheciis periphericis. (Pulvinatae Fries)

2996. Iph, concentrica Bolt. Op doode boomtronken. Aut. (F r. Syst. II. p. 331; Rabenh. p. 222)

In Herb.: 't Haagsche Bosch, B isch. (v. s.)

2998. Sph. Fragiformis Pers. $0 p$ de schors ran doode Beuken. Aut. Vere. (Fr. Syst. II. p. 332; Rabenh. p. 222)

In IIerb.: Renkom, Buse; in den Aardenhoul, Wolk. (v, so) 
29g5. Sph. fusea Pers. $O_{p}$ doode takben van Lizen en Beuken. Aut. Vere. (F r. Syst. II. p. 232; R abenh. p. 221; Dz. et M b. in Bijdr. fl. v. Ned. I. p. 19)

In Herb.: Leiden, Stekh.; Dz. et $\mathbf{H l b . ;}$ v. d. S. Lac.; Voorhout en Katwịk, W $t$ tw.; Amsterdam, Utrecht, v. d. S. La c.; Kampen, R. Bond.; ZuidBeveland, v. d. B.; Maastricht, F r a n q.

2999. Sph. argillacea Fr. $O p$ doode takken van IIazelaars en Elzen. Aut. (Fr. Syst. II. p. 333; Rabenh. p. 221; Dz. et II b. Bijdr. I. p. 19)

In Herb.: Leiden, $\mathbf{D}$ z. et $\mathrm{MI}$ b. ; Zuid-Beveland, v. d. B.; Maastricht, Fran q. (v. s.)

BOQ0. Sph. cohaerens Pers. $0 p$ doode boomtronken, bijzonder van Wilgen. llieme et Vere. (Fr. Syst. II. p. 333; Rabenh. p. 221; Dz. et II b. Bijdr. I. p. 19) In Herb. : Leiden, Dz. et $\mathbb{M l}$ b.; 0 udem. (v. s.)

BDO1. Iph. granulosa Pers. $0 p$ de schors van doode tronken van Crataegus Oxyacantha? Aut. Vere. (Fr. Syst. II. p. 335; R abenh. p. 221)

In Herb.: Leiden, W $t \mathrm{t}$ w. ; Bommel, J u n g h. (v. s.)

3002. Sph. multiformis Fr. Op doode Berken- en Elzenstammen. Vere. (Fr. Syst. II. p. 334; Rabenh. p. 221) Variat:

a. junior F r. I. I. In Herb.: Leiden, $\mathrm{W} t \mathrm{tw}$. (v. v. et s.) B. adulta Fr. 1. 1. In Herb.: Utrecht, Wttw. (v. v. et s.)

* * Stroma late effusum, indeterminatum, immarginatum, planum, perithecia cingens l. e. peritheciis connascentibus ortum. Perithecia collo destituta, in superficie immersa 1. prominentia. (Connatae Fr.)

3003. Sph. serpens Pers. In holle Wilgenstammen. Iliemc. Vere. (F r. Syst. II. p. 341 ; Raben h. p. 249) In Ilerh.: Bommel, J u ng h.; Zuid-Beveland, v. d, B. (v. v. et s.) 
3004. Sphr. confluens Tode. In vermolmde Wilgenstammen. Vere. Aut. (Fr. Syst. II, p. 342; R a hen h. p. 218)

In Herb.: Leiden, Dz. et II b.; Ankeveen (N. IIoll.), v. d. S. La c. (v. so)

Sect. 2. 埴yophericae. Compositae. Perithecia verticalia, immersa, stromate tecta, in collum attenuata. *** Determinatae, a matrice discretae. Perithecia immersa, ampla. (Glebosae Fr. 1. 1.)

3005. Th. Meusta $H$ of $\mathrm{fm}$. $0 \mathrm{p}$ doode Wilgentronken. Hieme. (Fr. Syst. pag. 345 ; Rabenh. p. 218)

In Herb.: Leiden, D z. et II b.; Haarlem, B r u g m。; Doornwerth, Buse; 't Soerensche Bosch, vo d. S. Lác. (v. s.)

3006. 7. Nummularia Dece $O_{p}$ doode takken van Eiken, Beuken en andere boomen. Per totum annum. (Fr. Syst. Il. p. 348; Rabenh. p. 217)

Maastricht, Franq. (n. v.)

×*** Determinatae, cum matrice connatae, basi linea nigra circursscriptae. Perithecia demersa, stipata. (Lignosae $\mathrm{Fr}$.)

300\%. W. Bullata Ehrh. $0 p$ doode takken van Wilgen, Elzen en Hazelaars. Vere. (Fr'; Syst, II, p. 349; li abenh. p. 216)

In Herb.: Bommel, Jungh.; Groningen, Strat.; De Heikop, De Il a an; Kampen, R. Bond,; Ubbergen, A bel. - Bovendien: Maastricht, Franq. (v. v. et s.)

300S. S. undulata Pers. $O p$ afgevallen doode takken van Cratnegus en Corylus. Vere. (Fr. Syst. II. 350; Rabenh. p. 216):

In Herb.: Leiden, W $t$ w。; Austerdaw, v. d, S. Lac. (ves.) 
3009. \$. Stigma 11 of $\mathrm{fm}$. Op doode takken van Esschen, Beuken en wilde Pruimen. Vere. (F r. Syst. II. p. 350; Rabenh. p. 216; D z. et MI b. Bịdr. I. pag. 19j

In Herb.: Utrecht, Haarlem, W $\mathrm{t} t \mathrm{w}$; Leiden, $\mathrm{D} z$. et $M l$ b. - Bovendien: Maastricht, F ranq.

3010. S. decorticata Dec. $0_{p}$ oude Beuken en Eiken. Per totum annum. (S. Stiggma $\gamma$ decorticata Tr. Syst. II. p. 350 ; Rabenh. p. 216)

In Herb.: Leiden, $\mathrm{D}_{\mathrm{z}}$ et $\mathbb{M} \mathrm{L} \mathrm{b}_{\mathbf{}}$; $\mathrm{W} \mathrm{tt} \mathrm{w}$; ; Bommel, J ungh.; Maastricht, Franq. (v. s.)

3011. S. diseiformis II o f $\mathrm{m}$. $O p$ doode takken van Beuken en wilde Kastanjes. Vere. (F r. Syst. II. p. 353 ; Rabenh. p. 216).

In Herb.: Leiden, W $\mathrm{ttw}$. - Bovendien: Mlaastricht, Franq.

2012. 5. verruciformis $\mathrm{Ehrh}$. Op takken van Carpiuus betulus, van Beuken en van IIazelaars. Aut. Vere. (Fr. Syst。 II. p. 355; Rabenh. p. 215)

In Herb.: Utrecht, $\mathrm{W}_{\mathrm{t} t \mathrm{w}}$ - Bovendien: Maastricht, Franq. (v. v.)

3013. 5. flavo-virens Pers. Op afgevallen doóde takken: IIieme. Vere. (F r. Syst. II. p. 357; R ab en h. p. 215) In Ilerb.: Utrecht, v. d. S. L a c.

5 * Determinatae, connatae, nulla linea circumscriptae. Perithecia sparsa. (Versatiles Fr.)

3014. S. Scabrosa Dec. $0 p$ doode Eikentronken en takken. Vere. (Fr. Syst. II. p. $360 ;$ R abenh. p. 215).

In Herb.: Mastricht, Fran q.: (v. s.)

34015. Ф. quereina P e rs. Op doode Eikentakken. Ilieme. Vere. (Fr. Syst. II. 362; R abenh. p. 214; Dz. et II b. Bijdr. I. p. 19)

In Herb.: 't Haagssche Bosch, W $t \mathrm{t}$ w.; Leiden, $\mathrm{D} \mathrm{z}_{\mathrm{o}}$ et $\mathrm{MI}$ b.; Utrecht, v. d. S. Lac. - Bovendien: Mlaastricht, Franq. (v. s.) 
3616. S. Laneiformis $\mathrm{Fr}$. $O_{p}$ doode Berkentaken, Lui. Vere. (Fr. Syst. II. p. 362; Rabenh. p. 214)

3018. S. ferruginea Pers. $O p$ doode takken van Hazelaars. Per annum. (Fr. Syst. Il. p. 363; R abenh. p. 211) In Herb.: Maastricht, Franq. (v. s.)

3018. 5. ceratosperma Tod. $O p$ doode Eikentakien. Aut. Vere. (Fr. Syst. II. p. 364; Rabenh. p. 211)

In Ilerb.: Hlarderwijk, J u $\mathrm{ngh.}$ - Bovendien: Maastricht, Franq. (v. v. et s.)

3019. S. Strumella Fr. $0 p$ doode takken van Aalbeziën. Vere. (Fr. Syst. II. p. 365; Rabenh.. p. 214)

In Herb.: Zuid-Beveland, v. d. B. (v. s.)

6 * Effusae, indeterminatae, connatae, haud circumscriptac. (Concrescentes $\mathrm{Fr}$.)

BO20. S. Spiculosa Pers. Op doode takken van Viburnum Opulus. Vere. (Fr. Syst. II. p. 369; Rabenh. p. 213)

In Herb.: Jtrecht, Wttw.

3021. S. lata Pers. $O_{p}$ doode Esschenschors en hout. Vere. (Fr. Syst. II. p. 369; Rabenh. p. 213; Dz. et $\mathrm{II}$ b. Bịjdr. I. p. 20)

In Herb.: Leiden, $\mathrm{D} z$. et $M \mathrm{H}$.; Utrecht, W $\mathrm{t}$ w.; Amsterdam, v. d. S. Lac.; Zuid-Beveland (Goes); v. d. B. - Bovendien : Haastricht, Franq. (v. s.)

302. 5. velutina $W$ all r. $O_{p}$ doode afgevallen takken en hout. Vere. (R a benh. p. 213)

In Herb.: Zuid-Beveland, vo d. B.; Leiden, D z. et Mi b.

3023. 5. salicella $\mathrm{Fr}$. Op doode Wilgentakken. Aut. Vere. (F r. Syst. II. p. 377)

In Herb.: Amsterdam, v. d. S. Lac.; Zuid-Beveland (Goes), v. d. B. (v. s.)

Sect. 3. Amphiphericac. Compositae. Perithceia con- 
vergentia, circinantia, stromale spurio undique pusta, lato-cincta, in collum attenuata,

7 * Perithecia in conceptaculo integro, ab epidermide discreto, collecta. (Circumscriptae Fr.)

3024. \$. gastrina $\mathrm{Fr}, 0 p$ doode tronken van 0 lmen en Haagbeuken. Aut. Vere. (F r. Syst. II. p. 379; Kabenh. p. 210)

In Herb.: Harderwijk, Jungh.

3025. \$. IPunastri Pers. $0 p$ doode Pruimtakken. Aut. Vere. (Fr. Syst. II. p. 380 ; Rabenh. p. 212)

In Herb.: Utrecht, $\mathrm{W} t \mathrm{t}$ w.

3026. \$. stellulata Fr. Op doode Olmtakken. Aut, (Fr. Syst. pag. 380)

In Herb.: Amsterdam, v. d. S. La c.

3028. .. Lim minghiil W e s t. Op doode Olmtakken. Verc.

Perithèces petits, sphériques, noirs, grroupés au nombre de 6 à 15, nichés entre les fibres corticales et surmontés chacun d'un col cylindrique plus ou moins long. Les ostioles de chaque groupe se réunissent en convergeant pour soulever et percer transversalement l'épiderme, sous forme d'un disque arrondi, proéminent, mamelonné, noir et luisant. Thèques en massue presque cylindrique ou fusiforme, sans paraphyses, longues de 12 a $15 / 100$ de mill. Sporidies uni- ou bisériées, fusiformes, hyalines, de $3 / 100$ es de mill. de longueur sur $1 / 100^{\circ}$ de mill. de largeur, et n'offrant à l'intérieur aucune trace de cloison, mais seulement quelques granules peu distinets. (West. 6e not. Crypt. Nouv. de la fl. Belge $n^{0} .26$, in Bull. de l'Acad. Roy. des Sc. de Belgique, $2^{e}$ Série Tom. VII.) Obs. Verschilt grootelijks in de vruchtdeelen van de voorgaande.

In Herb.: Amsterdam, v. d. S. Lac.; Leiden, Ankers m.; Groningen, v. d. S. Lac. (v. v. et s.)

302S. S. enteroleuea Fr. Op dnorle afgevallen Fiken- 
takken. IIieme. Vere. (Fr. Syst. II. p. 381; Rabenh. p. 210)

In Herb.: Harderwijk, Jungh.; De Vriese; Leiden, $\mathrm{Dz}$. et II b. ; 't Haagsche Bosch, v. d. S. L a c. Bovendien: Maastricht, Franq. (v. v. et s.)

3029. Anomia Fr. $O_{p}$ doode takken van Robinin pseudo-Acacia. Aut. Vere. (Fr. Syst. II. p. 381; Rabenh., p. 210)

In Herb.: Harderwijk, Jungh. ( $v$, s.)

3030. S. Aletrusa Fr. Op doode takken van den Berberis. Aut. Vere. (Fr. Syst. II. p. 382; Rabenh. pag. 211)

In Herb.: Leiden, W $t \mathrm{t}$ w. (v. 8.)

3031. . Carpini Pers. $O p$ doode takken van Carpinus Betulus. Aut. (Fr. Syst. II. p. 384 ; R a ben h.p. 210) In Herb.: 's Hertogenbosch, W e st.

8 * Perithecia in conceptaculo dimidiato et superne epidermide adnata inclusa, disco erumpente. (Incusae Fr.)

3032. S. mivea II of $\mathrm{fm}$. Op jonge doode Populier-en Wilgentakken. Per totum annum. (Fr. Syst. II. p. 386; R abenh. p. 209)

In Herb.: Amsterdam, 0 u dem. ex. herb. Bragm. (v. s.)

3033. 5. leucostoma $\mathrm{Fr}$. Op doode Pruimtakken. Per annum. (Fr. Syst. II. p. 387; Rabenh. p. 209) Variat:

a. polypora Fr. l. I. In Herb.: Bommel, J ungh. (v. s.)

3031. S. controversa Desmaz. $0 p$ doode takjes van Cornus sanguinea, Acer Negundo, Fraxinus. Hieme. Aut.

Circumscripta, stromate corticali nig̣ro. Peritheciis immersis, ostiolis erumpentibus prominulis. Ascis snho 
clavatis; sporidiis biseriatis, hyalinis, ovato-oblongis; sporulis 4, globosis. (Desmaz. 9e Not. pl. crypt, de Fr. no. 18 in Ann. des Sc. Nat. Févr. 1842). Variat:

a. corni West. Herb. Crypt. Belge, no. 910.

In Herb.: Leiden, v. d. B. ( $\left.\mathbf{v}: \nabla_{0}\right)$

* 9. Perithecia absque conceptaculo proprio in pustula corticali ostiolaque in discum collecta (Obval. latae $\mathrm{F} \mathrm{r}_{\text {o) }}$

3035. Ф. coromate $\mathrm{H}$ offm. Op schors van Beukentakken. Aut. Vere. (Fr. Syst. II. par. 395; R a be $\mathrm{n}$ b. p. 204)

In Ilerb.: Utrecht, W $t \mathrm{tw}$.

3036. 5. decorticams $\mathrm{Fr}$. Op afuestorven schors ran Fagus sylvatica en Sorbus aucuparia. Hieme et Vere. (Fr. Syst. II. p. 396; R a benh. p. 207)

Mlaastricht, Fran q. (n, v.)

3938. 5. teurgida Pers. Op doode Beukentakken. Aut. Vere. (Fr. Syst. II. p. 400; Rabenh. p. 206) In Herb.: Maastricht, Franq. (v. s.)

3035. פ. Salieina Pers. $O p$ doode Wilgentakken. Aut. (Fr. Syst. II. p. 401; Rabenh. p. 206)

In Herb: : Rotterdam, $\mathrm{O}$ udem.; Leiden, W $\mathrm{t} t$ w.; Zuid-Beveland (Goes), r. d. B. (v. v. et s.)

3039. פ. stilbostoma Fr. $0 p$ doode takken ran verschillende boomen. Aut. (Fr. Syst, II. p. 403; Rabenh. p. 205). Varia: :

a. umbiliceta Fr. 1.I. Op doode takken van Acer pseudo-platanus.

In Herb.: Amsterdam, v. d. S. La c. (v, s.) 
* 10. Perithecia in orbem disposita, libera absque conceptaculo et disco (Circinatæ F r.)

3040. פ. pullchella Pers. Op afgestorven Pruimenschors. Hieme et Vere. (Fr. Syst. II. pag. 406; Rabenh. p. 204)

In Herb.: Maastricht, Franq. (v. vo et s.)

3011. Conjuncta $\mathrm{N}$ e es. Op doode stengels van Braambeziën.Aut. Vere. (F r. Syst. II. p. 408; R a b e n h. p. 204) In Herb.: 't Haagsche Bosch, Dz. et II b. (v. s.)

3442. quaternata Pers.? Op oude Berken- en Beukenschors. Aut. Vere. (Fr. Syst. II. p. 409; R abenh. p. 203)

In Herb.: Walcheren (duinkant), v. d. B. (v. s.)

Sect. 4. Epiphericae. Subcompositae. Perithecia horizontalia, supra stroma (spurium saepius) nuda collocata, sed primo a matrice tecta, collo nullo.

* 11. Perithecia caespitosa, superficialia, supra stroma subinnatum erumpens collocata (Caespitosae Fr.)

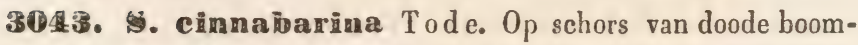
stammen. Hieme. Vere. (F r. Syst. II. p. 412; Rabenh. p. 202; Dz. et II b. Bịjdr. I. p. 20.)

In Herb.: Leiden, $\mathrm{D} z$. et $M b_{\text {., }}$ de $\mathrm{Ha} \mathrm{a}, \mathrm{W} t \mathrm{tw}$; Kampen, R. Bond.; Zuid-Beveland, v. d. B.; Groo ningen, Stekh., Strat.; Amsterdam, v.d.S. L ac.; Maastricht, Franq. (v. v. et s.)

394. 9. coceinea Pers. Op doode Pruimen-en Elzenstammen. Ilieme (F r. Syst. II.p. 412; R a be n h. p. 202; Dz. et M b. Bijdr. I. p. 20.)

In Ilerb.: Zuid-Beveland, v. d. B.; Amsterdam, v. d. S. La c. - Bovendien: Maastricht, Franq. (v.v. et v.). Variat: 
a. sanguinella Fr. I. I. In Herb.: Leiden, $\mathbf{D} z$, et Mb. (v, s.)

3045. T. ribis Tode. $0 p$ doode Aalbezietakken. Aut. Vere. (Fr. Syst. II. p. 413; Rabenh, p. 202; Dz. et II b. Bijdr. I, p. 20)

In Herb.: Leiden, Dz, et Ml b.; Haastricht, $\mathrm{Fr}$ a nq; ; Zuid-Beveland, v. d. B.

3046. \$. laburni Pers. 0 p doode takken van Cytisus laburnum. Aut. Vere. (Fr. Syst. II. p. 413; R aben h. p. 202; Dz. et 11 b. Bijdr. II. p. 17)

In llerb.: Leiden, $\mathrm{Dz}$. et $\mathrm{Hl}$ b. (v. s )

3048. 9. acervalis II o u g. $0 p$ doode Esschen- en Wilgentakken. Aut. (F r. Elench. II. p. 83; R a ben h.p. 202)

In Herb.: Amsterdam, v. d. S. L a c. - Bovendien: Maastricht, Franq. (v. s.)

3048. 5. cyanogena Desma $z, 0$ p roltende Koolstronken. Aest. Aut.

Peritheciis minutissimis, numerosis, conglomeratis, raro solitariis, globosis, rugosis, atris, opacis; ostiolo papillæformi; nucleo albido; ascis clavatis; sporidiis hyalinis, oblongis, subfusiformibus, curvulis, vix obtusis, 3 septatis. (Desmaz. 15e Not. crypt. de Fr. no. 18 in Ann. Sc. nat. $3^{\mathrm{e}}$ Série. Tom. IX p. 352 ; Desmaz. Pl. crypt. de Fr. no. 2060)

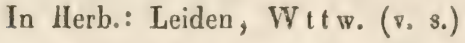

3049. T. berberidis $\mathrm{P}$ ers. $\mathrm{Op}$ doode takken van Berberis vulgaris. Per annum. (Fr. Syst. II. p. 415; Rabenh. p. 201; D z. et II b. Bijdr. III. p. 8)

In Herb.: Leiden, de $\mathrm{H}$ a an, $\mathrm{D} z$. et $\mathrm{II} b$., W $\mathrm{t} t \mathrm{w}$; Utrecht, Amsterdam, v. d. S. La c. (v. v. et s.)

3050. ฐ. varia Pers, Op afgestorven Pruimenschors (Fr. Syst. II. p. 416; Rabenh. p. 201)

Maastricht, Franq. (n, v.) 
3051. 5. cupularis I'ers. $0 p$ drooge Pruimen- en Vliertakken, en die van andere boomen. Hieme. Vere. (Fr. Syst II. p. 416)

In Herb.:Leiden, W $\mathrm{t} t \mathrm{w}$; Zuid-Beveland (Goes), r.d.B.

3052. 5. pulicaris $\mathrm{Fr}$ ? $0 p$ afgestorven Vliertakken. Aut. (Fr. Syst. II. p. 417; Rabenh. p. 200; Dz. et II b. Bijdr. I. p. 20)

In Herb.: Leiden, $\mathrm{D} z$, et $\mathrm{II} b$.

3053. 9. agregata $\mathrm{L}$ as ch, 0 p doode stengels van $E u$ phrasia odontites.

Peritheciis erumpentibus subrotundis nigris nitidis, seriatim aggregatis; nucleo albo; ascis subclavatis octosporis; sporis cylindraceis monoblastis aut unisepta= tis (Lasch in Rabenh. Herb. viv. myc. Ed. II. $\left.n^{\circ} .541\right)$

In Herb.: Zuid-Beveland, v. d. B. (vo s.)

* 12. Perithecia primo immerso-innata, demum emersa, confluentia (Confluentes $\mathrm{F}_{\text {r. }}$ )

3054. \$. Clongata Fr. Op doode takken van Robinia pseudo-acacia. Aut. Vere. (Fr. Syst. II. p. 422; Rabenh. p. 199)

In Herb.: Leiden, W $t \mathrm{tw}$. (

3055. F. mucosa Pers. Op rottende vruchten van Cucurbita pepo. Vere. (Fr. Syst. II. p. 425; R a ben h. p. 198; Dr. et $H \mathrm{l} b . \mathrm{Bijdr}$. I. p. 20)

In Herb. : Leiden. D p et $M / \mathrm{b}$.

3056. Ф. dothidea II o g. Op doode Esschentronken en takken. Aest. Aut. (Fr. Syst. II. p. 423; Rabenh. p. 200). Variat:

a. Rosae Fr. Elench. II. p. 86, 0p levende Rozentakken. In Herb.: Hulst, West. (v. v.)

395\%. 9. Spartii Necs, $O p$ doode tronken en takken van 
Sarothamnus vulgaris (Fr. Syst. II. p. 424; Rabenh. p. 199)

In Herb.. Axel, W est.

* 13. Perithecia in series parallelas disposita (Seriatae $\mathrm{Fr}$.)

3058. T. rimosa Alb. et Schw. $0 p$ drooge halmen van Phragmites communis. Aut. Hieme. (Fr. Syst. II. p. 427; R abenh. p. $190 ; \mathrm{D}$ z. et II b. Bijdr. I. p. 20)

In Ilerb.: Leiden, Dz. et MIb.; Voorschoten, d e $\mathrm{H}$ a a ; Utrecht, W $\mathrm{ttw}_{\mathrm{w}}$ - Bovendien: Maastricht, Franq. (

3059. 5. fillicina Fr. Aan de bladstelen van Varens. Vere. (F r. Syst. II. p. 427; R abe $\mathrm{nh}$. p. 190)

In Herb.: Utrecht, J u n g h.; Haastricht, F r a n q. (v. s.)

3060. \$. junei Fr. Op drooge halmen van Juncus glaucus. Aut. (Fr. Syst. II. p. 428; R aben h. p. 191)

In Herb.: Leiden, $\mathbf{D}_{z}$, et $\mathrm{M}$ b.; Zuid-Beveland, v. d. B.; Utrecht, W ttw. (

3061. 5. lirrella Houg. $O_{p}$ doode stengels van Spiraea ulmaria. Aut. (Fr. Elench. II. p. 105; Rabenh. p. 192; D r. et II b. Bijdr. II. p. 17)

In Herb.: Blauwkapel, จ. d. S. Lac.

3062. S. arundinacea $\mathrm{S} o w . \quad \mathrm{p}$ drooge halmen van Phragmites communis. Vere. (Fr. Syst. II. p. 429; R abenh. p. 193; Dz. et II b. Bijdr. I. p. 20)

Subtecta, linearis, stromate vix distincto. Peritheciis minutis, globosis, uniserialibus, subastomis, nigro farctis. Ascis nullis; sporidiis elongatis, subfusiformibus; sporulis 4-6, semiopacis (Desmaz, emend. 12e Not, crypt. de Fr. in Ann. des Sc. nat. Janv. 1846.p. 49) In Herb.: Leiden, Stekh., W $t \mathrm{t}$.

3063. \$. godini Desmaz. Als de voorgaande. Vere. Subtecta, oblonga, minuta, griseo-nigra, stromate 
vix distincto. Peritheciis minutissimis, stipatis, compressiusculis, 2-3 serialibus, subastomis albo farctis. Ascis clavatis; sporidiis oblongis, 3 septatis. (D e s maz. Loc. cit.)

In Herb.: Leiden, Stekh., W t tw.; Amsterdam, v. d. S. L a c.

3064. \$. mebullosa Pers. Op doode stengels van groene gewassen en bijzonder van Aardappelen. Hieme. (F r. Syst. II. p. 430 ; Rabenh. p. 191 ; S. nebularis $\mathrm{Dz}$. et $\mathrm{Ml}$ b. Bijdr. III. p. 8)

In Herb.: Leiden, v. d. S. L a c. - Bovendien: Maastricht, Franq, (v. s.)

3065. Ф. polygramma $\mathrm{F}$ r. Op doode stengels van Angelica sylvestris. Vere. (Fr. Syst. II. p. 432; Rabenh. p. 192)

In Herb.: Leiden, W $t \mathrm{t}$ w. - Bovendien: Milastricht, Franq. (v. s.)

* 14. Perithecia aggregata, foliis innata (Confertae $\mathrm{Fr}$.)

3066. \$. graminis Pers. Op doode bladen van verschillende grassoorten. Aut. Vere. (F r. Syst. II. p. 434; Rabenh. p. 189; Dz. et II b. Bijdr. I. p. 20)

In Herb.: Leiden, $\mathbf{D} z$, et $\mathrm{Ml}$ b。 - Bovendien: Maastricht, Fran q.

306\%. ๑. ซrifolii Pers, Op de bladen van Trifolium repens. Aat. (Fr. Syst. II. p. 435; R a benh. p. 188)

In Herb.: Leiden, Dz. et II b., O udem.; Zwake, v. d. B.; Beuningen bij Nijmegen, A bel.

3068. 5. allicina Fr.? Op stervende bladen van Allium Porrum. Aat. Hieme. (F r. Syst. II. p. 437; D z. et MI b. Bijdr. I. p. 20)

In Herb.: Leiden, $\mathbf{D} z$, et $\mathbb{1} \mathrm{b}$.

3069. $\$$, bifrons Schmidt et Kzce Op verdroogde Ei- 
kenbladen. Vere. (Fr. Syst. II. p. 438; R a benh. p. 189)

In Herb.: Leiden, in den Hortas Academicus, $\mathrm{D}_{z}$. et $\mathrm{II} b ., \mathrm{W} t \mathrm{tw}$.

3080. \$. ceuthocarpa $\mathrm{Fr} .0 p$ verdroogde bladen van Populus tremula. Aut. Vere. (Fr. Syst.p. 439 ; R abenh. p. 189)

In Herb.: Zuid-Beveland, v. d. B.; Wassenaar, W ttw. - Bovendien: Mlaastricht, Franq. (v. vo et s.)

\section{H. I im I ices.}

Sect. V. Superficiales. Simplices. Perithecia bicorticata supra stroma effusum villosum I, matricem plane superficialia, libera, primo velata.

* 15. Perithecia per se glabra, insidentia subiculo tomentoso (Byssisedae Fr.)

30\%1. 5. aurantia Pers, Op rottende kurkachtige $\mathrm{P}_{0}$ lypori. Per annum. (Fr. Syst. II, p. 440; Rabenh. p. 198)

Maastricht, Fran q. (n. v.)

308\%. \$. aquila Fr. $O_{p}$ doode en rottende boomtakken. Hieme et Vere (Fr. Syst. II. p. 442 ; Raben h. p. 197) In Herb.: Utrecht, vo d. S. La c.; Maastricht, Franq. (v. s.)

3083. F. Byssiseda Tode. Op rottende Wilgentakken. Vere (Fr. Syst. II. p. 442; Rabenh. p. 197) Maastricht, Franq. (n, v.)

3084. 5. tristis Tode, Op rottend hout van Acer pseudo-platanus. Vere. (Fr. Syst, II. p. 444; R a benh. p. 197)

In Herb, : Utrecht, Wttw. (v, 8.) 
* 16. Perithecia villo persistente tecta (Villosae Fr.)

30\%5. \$. ovina Pers. $0 p$ roltend Wilgenhout in holle stammen. Hieme. (F r. Syst. II. p. 446; R a b e n h. p.196)

In Herb.: Willemspolder, $\mathbf{D}$ z. et $\mathbb{M}$ b. (v. 8.).

3086. Wirsuta Pers. Op rottend hout. Aut. Hieme. (Fr. Syst. II. p. 449; R a b enh. p. 194)

In Herb.: Zuid-Beveland (Goes), v. d. B. (v. v. et s.)

* 17. Subicalum nullum. Perithecia glabra, basi rotundata, sublibera, ostiolo persistente (Denudatae Fr.)

308\%. Ф. peziza Tode. $0 p$ rottend hout. Aut. Vere. (Fr. Syst. II. p. 452; Rabenh. p. 190)

In Herb.: Amsterdam, vo d. S. L a c. - Bovendien: Maastricht, Franq? (v. s.) Variat:

a. villifera Fr. 1. 1. (Sph. peziza $\beta$. miniata Rabenh.1.1.) Maastricht, Fran q. (n. v.)

30\%8. ฐ. sanguinea $\mathrm{Sibth}$. Op verdroogd Elzenhout. Aut. Vere. (Fr. Syst: II. p. 453; Rabenh. p. 174)

In Herb.: Rotterdam, $\mathrm{O}$ u dem. (v. s.)

3089. \$. episphaeria Tode. $0 p$ de puisten van Sphaeria quercina, stigma en anderen. Hieme. Vere. (Fr. Syst. II. p. 454; Rabenh. p. 190).

In Herb.: de bosschen van Ameliswaard bij Utrecht, v. d. S. L a c. (v. v.)

3080. \$. mammaeformis Pers. Op weggeworpen rottend hout. Hieme et Vere. (Fr. Syst. II. p. 455; R abenh. p. 176)

In Herb.: Zwake, จ. d. B. - Bovendien: Maas: tricht, Franq. (v. s.)

3081. ฐ. stercorarla Sow. Op paardenmest. Hieme. (F r. Syst. II. p. 455; Rabenh. p. $174 ; \mathrm{D}$ z。 et $\mathrm{Ml}$. Bijdr. I. p. 20)

In Herb: : Harderwijk, J ungh.; Leiden, $\mathrm{Dz}$, et II b. ( $v_{i}$ s. $)$ 
3082. Ф. obducens $a$. minor $F_{r}$. $O_{p}$ doode takken van Fraxinus excelsior. Aut. Vere. (Fr. Syst. p. 456; Rabenh. p. 176)

In Herb.: Utrecht, W $t \mathrm{tw}$ ( (v. s.)

3093. 5. bombarda Batsch. Op rottende boomstammen. Aut. Vere. (Fr. Syst. II. p. 456 ; R abeah.p. 177) Maastricht, Fran q. (n. v.)

3084. \$. spermoides Fr. Op rottende Eiken- en Wilgenstammen. Ilieme. Vere. (Fr. Syst. II. p. 457; Rabenh. p. 176)

In Herb.: Utrecht, Dz. et II b.; Zuid-Beveland, v. d. B.; Amsterdam, v, d. S. L a c. - Bovendien: Maastricht, Franq. (v. vo et s.)

3055. 5. moriformis $\mathrm{T}$ ode. In rottende holle Wilgentronken, alsook op rottende stammen van Prunus mahaleb. Per annum. (Fr. Syst. II. p, 458; R a ben h. p. $176 ; \mathrm{Dz}$. et $\mathrm{MI}$ b. Bijdr. II. p. 17)

In Herb.: Zuid-Beveland (Goes), v. d. B.; Leiden, W $t \mathrm{tw}$; Kampen, R. B ond. (v. v. et s.)

3086. 9. erubescens $\mathrm{R} o \mathrm{~b}$. $\mathrm{O}_{\mathrm{p}}$ doode bladen van Ilex aquifolium. Aut.

Sparsa vel subgregaria, superficialis, basi villosa alha radiata. Peritheciis minutis, globosis, mollibus, glabris, testaceis dein badio-rubris coilabescendo concavis. Ostiolo papillaeformi. Ascis clavatis; sporidiis oblongis subacutis, subcurvulis, hyalinis, 3 septatis (Rob. in Desmaz, 13e Not. erypt. de Fr. pag. 72)

In Herb.: Zuid-Beveland, v. d. B. (vos.)

308\%. pulvis pyrius $\mathrm{P}$ ers. 0 p vochtig rottend hout. Vere. (Fr. Syst. II. p. 458; Rabenh. p. 176; D z. et $M$ b. Bijdr. I. p. 20)

In Herb.: Leiden, Dz. et II b.; Rijnsburgerweg, de $\mathrm{H}$ a an; Leiden en Utrecht, Wttw., v. d. S. Lac.; Muyden, Uyleupas, Socrensche bosch, v, d. S. Lac; 
Zuid-Beveland (Goes), v. d. B. - Bovendien: Mĩastricht, Franq. (v. vo et s.)

3085. ฐ. pulveracea $\mathrm{Ehrh}, 0 p$ dood en rottend hout. Hieme ct Vere. (F r. Syst. II. p. 459 ; R a b e n h. p. 174) In Herb.: Amsterdam, v. d. S. La c: (v. v. et. s.)

* 18. Perithecia glabra, basi applanata subinnata pertusa (Pertusae Fr.)

3089. 5. mastoidea Fr. Op doode Wilgen- en Esschentakjes. Per annum. (Fr. Syst. II. p. $463 ; \mathrm{D}$ z, et IIb. Bijdr. I. p, 20)

In Herb.: Leiden, Dz, et II b.; de Bild, v. d. S. Lac. ; Haarlem (v. s.)

3090. 5. pertusa Pers. $0 p$ rottend hout. Vere. (Fr. Syst. II. p. 464; R a benh. p. 178; D z. et $\mathbb{I I}$ b., Bijdr. I. p. 20)

In Herb.: Leiden, Dz. et II b.; Zuid-Beveland, v. d. B. (v. s.)

Sect. VI. Subimmersae. Simplices. Perithecia immersa, saepe erumpentia, ostiolo insignni dilatato 1. in collum elongato.

* 19. Ostiolum latissimum, compressum, rima longitudinali dehiscens (Platystomae F r.)

30D1. ๑. excipuliformis $\mathrm{Fr}$. $\mathrm{O}_{\mathrm{p}}$ oude Eikenschors. Per annum. (Fr. Syst. II. p. 469; R a benh. p. 184)

In Herb.: Leiden, Dz, et Hb; Utrecht, Wtt.w.; Zaid-Beveland, v. d. B. (v. s.)

3092. \$. macrostoma Tode. $O_{p}$ afgevallen takken, dood hout en oude schors van boomen. Aut. Verę. (Fr. Syst. II. p. 469; R a benh, p. 184)

In Herb.: op den Rijnsburgerweg; de $\mathrm{H}$ a an (v. 8.) 
* 20. Ostiolum elongatum, cylindricum, liberum, perithecio longius (Ceratostomae Fr.)

3093. 9. rostrata $\mathrm{Fr}, \mathrm{Op}_{\mathrm{p}}$ doode en roltende Beuken- en Berkentakken. Per annum. (Fr. Syst. II. p. 473; Rabenh. p. 186)

In Herb.: Amsterdam, v. d. S. La c. (v. s.)

3094. 5. rostellata $\mathrm{Fr}$. $0 \mathrm{p}$ doode stengels van verschillende Rubus-soorten. Aut. Vere. (Fr. Syst. II. p. 476; Rabenh. p. 186)

Haastricht, Fran q. (v: v.)

* 21. Ostiolum in collum matrici immersum elongatum (Obtectae Fr.)

3095. ๑. eutypa Fr. $0 p$ het hout van doode boomtronken. Per annum (Fr. Syst. II. p. 478; Rabenh. p. 183; Dz. et M b., Bijdr. I. p. 20)

In Herb.: Leiden, $\mathbf{D} z$. et $\mathbb{M}$ b. (v. s.)

3D96. .. opercullata $A$. et $S_{\text {; }}$ Op rottend Dennen- en Elzenhout. Hieme et Vere. (Fr. Syst. II. p. $479 ; \mathrm{D} z$. et $\mathrm{II}$ b., Bijdr. I. p. 20)

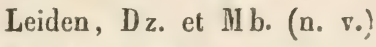

3098. S. corticis Fr. $0 p$ doode Populierschors. Aut. et Vere. (Fr. Syst. II. p. 481; Rabenh. p. 182) Maastricht, Franq. (n. v.)

3098. Ф. đitopa Fr. Op doode Elzentakken. Aat. et Hieme. (Fr. Syst. II. p. 481; Rabenh. p. 182)

In Herb.: Warmond, de Haan (v. s.)

30D9. \$. tillae Fr. Op doode en afgevallen Lindentakiten. Vere. (Fr. Syst. II. p. 485; Rabenh. p. 181)

In Herb.: Leiden, A n kers.; Haarlem (vo s。)

3100. Truinosa Fr. $0 p$ de schors van doode Esschentakken. Aut, Vere. (Fr. Syst. II. p. 486; R a= benh.p. 182) 
In Herb.: Leiden, Ju ngh. - Bovendien: Maastricht, Fran q.

3101. 5. xylostei Pers. Op doode takken van Lonicer'a xylosteum. Vere. (Fr. Syst. II. p. 487; Raben h. p. 181)

In Herb.: Utrecht, W $t$ tw. (v. s.)

Sect. VII. Subinnatae. Simplices. Perithecia epidermidi matricis subinnata velo nullo, gelatina diu farcta.

* 22. Perithecia mox erumpentia, liberata, ostiolo simplici (Obturatae Fr.)

3102. ธ. sapinea $\mathrm{Fr}$ ? $O_{p}$ schors en takken van Dennen en Pijnboomen. Vere. (Fr. Syst, II. p. 491)

In Herb.: Harderwijk, J a ngh.

3103. \$. Syringae $\mathrm{Fr} .0 p$ doode takken van Syringa vulgaris. Vere. (Fr. Syst. II. p. 492 ; R a be n L. p. 179; Dz. et II b. Bịjdr. III. p. 8)

In Herb.: Zuid-Beveland (Goes), v. d. B. (v. s.)

3101. S. Ionicerae $S o w . ~ O p$ de doode stammen van Lonicera periclymenum. Vere. (Fr. Syst. II. 492; Rabenh. p. 179)

In Herb.: Utrecht, W $t \mathrm{tw}$. (v. s.)

3105. \$. Strobilina II oll, et Schm. $0 p$ de schubben van rottende Pijnkegels. Hieme. Vere. (F r. Syst. II. p. $495 ;$ D z. et M b. Bijdr. I. p. 20)

Leiden, $\mathrm{D} z$, et $\mathrm{MI}$ b. (n. $\nabla$.)

3106. 5. epieymatia $\mathrm{Wall}$. $0 \mathrm{p}$ de scutella van $\boldsymbol{P a}$. tellaria subfusca en parietina. Vere. Aest. (Rabenh. p. 180; Sph. Lichenicola Dz. et Mlb. Bijdr. II. p. 17)

In Herb.: Utrecht, v. d. S. Lac.; Zuid-Beveland (Goes), v. d. B. (v, ss) 
* 23. Perithecia primo innata, cum matrice concreta, demum supra denudata, ostiolo simplici (Subtectae $\mathrm{Fr}$.)

3110\%. epidermidis Fr. Op de talien van Berberis vulgaris. Per annum. (Fr. Syst. 1I. p. 499). Variat:

a. microscopica Desmaz. Op de zaadhuisjes van den Esch. Vere. (Desmaz. Pl. Crypt. de Fr. no. 975)

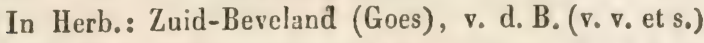

3108. 5. taxi Sow. $0 p$ de bladen van Taxus baccata. Vere. Aut. (Fr. Syst: II. p. 500, Rabenh. p. 171)

In Herb.: Zuid-Beveland, vo d. B. (vos.)

3109. \$. macularis Fr. Op stervende bladen van Acer en Populus. Aut. (Fr. Syst. II. p. 502; R abenh. p. 187)

In Herb.: Groningen, Strat. (ve s.)

\$110. T. illeis Fr. $0 p$ doode bladen van Ilex aquifolium. Aut. Vere. (F r. Syst. II. p. $501 ; \mathrm{D}$ z. et $\mathrm{MI}$ b. Bijdr. I. p. 20)

In Herb.: Leiden, $\mathrm{D}_{\mathrm{z}}$ et $\mathrm{li} \mathrm{b}$.

* 24. Perithecia primo tecta, demum epidermide annua discrela secedente nuda (Caulicolae Fr.)

3111. pellita Fr. $0 p$ doode stengels vas Papaver en andere kruiden. Aut. Hieme. (Fr. Syst. II. p. 505)

In Herb.: Warmond, de $\mathrm{Haan}$ (v. s.)

3112. S. rubella Pers. $0_{p}$ doode stengels van kruiden. Hieme. Vere. (Fr. Syst. II. p. 50 ; $\mathrm{R}$ a b $\in \mathrm{n}$ h. p. 179 ; Dz. et $M$ b. Bijdr. I. p. 20)

In Herb.: Leiden, Dz. et II b.; Amsterdam, v: d. S. Lac.; Haarlem. - Bovendien: Haastricht, Franq. (v. v. et s.)

3113. Haematites Desmaz, Op doode stengels van Clematis vitalba. Vere. 
Confertiu sparsa, minuta, macula sanguinea insidens. Peritheciis orbiculatis, primum pallescentibus, dein nigrris, humectatis convexis, siccis pezizoideo-collapsis, epidermide translucenti semper tectis. Ostiolo punctiformi. Ascis cylindrico.clavatis; sporidiis non observatis (Desmaz, $19^{\mathrm{e}}$ Not. pl. crypt. de Fr. $\mathrm{n}^{\circ} .24$ in Ann. des Sc. nat. 2e Série Tom. XVI.)

In Herb.: Zuid-Beveland, v. d. B.

3114. S. acuta II $\mathrm{f} f \mathrm{~m}$. Op doode stengels van Brandnetels (Urtica urens et dioica). Hieme. Vere. (Fr. Syst. II. p. 507; Rabenh. p. 175; Dz. et IIl b. Bijdr. III. p.8) In Herb.: Leiden, Dz. et II b.; Utrecht, v. d. S. I ac.; Amsterdam, v. d. S. Lac.; Leiden, W ttw.; Zuid-Beveland (Goes), v. d. B.; Warmond, de $\mathrm{H}$ a an; Maastricht, Fran q.

3115. 5. modesta Desmaz. $0 p$ doode stengels van groote kruiden; Aut. Vere.

Sparsa. Peritheciis globoso-depressis, minutis, epidermide primo tectis, dein nudis, nigris, laevibus, nitidis. Ostiolo papillato obtuso. Nucleo albo. Ascis amplis, subcylindricis; sporidiis fusiformibus, obtusiusculis, curvulis 4-6 septatis; sporulis minutissimis, globosis hyalinis. (Desmaz, $14 \mathrm{e}$ Not. crypt. nouv.

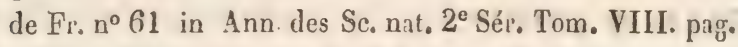
173. - Sph. complanata Lib. (non Tode)!)

In Herb.: Zuid-Beveland (Goes), ₹. d. B. (

3116. 5. coniformis $\mathrm{Fr}$. Op de doode stengels van groote kruiden, vooral van Brandnetels en Umbelliferae. Vere. (Fr. Syst. II. p. 508. - Sph. herbarum c. conica Rabenh. p. 175)

In Herb.: Leiden, $\mathbf{D} z$. et $M b_{\text {. }}, \mathrm{W} \mathrm{tt}$ w. Utrecht, Amsterdam, v. d. S. L a c.

Zuid-Beveland (Goes), v. d. B. (v. v. et s.)

311\%. ๑oldolum pers, $\mathrm{Op}_{\mathrm{p}}$ doode stengels van Umbel. 
liferae en voonal van Angelica sylvestris. Vere. (Fr. Syst. II. p. 509; Rabenh. p. 173; Dz. et II b., Bijdr. I. p. 21)

In Herb.: Leiden, Dz. et II b., W $t \mathrm{tw}$; Blauw. kapel, v. d. S. Lac.

Bovendien: Maastricht, Franq. (vo v. et s.)

3118. 5. pisi $\mathrm{S}$ ow. $\mathrm{O}_{\mathrm{p}}$ doode stengels van Pisum sativum. Ilieme. Vere. (F r. Syst. II. p. 509; R a b e n h. p. 173) In Herb.: Zuid-Beveland, v. d. B. (v. vo et s.)

3119. 5. caulium $\mathrm{Fr}, \mathrm{O}_{\mathrm{P}}$ uitgerukte stengुels van Umbelliferae. Vere. (F r. Syst. II. p. 509) Maastricht, Franq. (n, vo)

3120. \$. culmifraga $\mathrm{Fr} .0 p$ doode Grashalmen. Iliemc. Vere. (Fr. Syst. II. p. 510; R a benh. p. 180). Varial: b. linearis $\mathrm{Fr}$. I. I. $0 \mathrm{p}$ doode koornhalmen. In Herb.: Zuid-Beveland, v. d. B. (v. s.)

3121. . scarpicola Dec. Fr. Op doode bladen van Scirpussoorten. Hieme. Vere. (Fr. Syst. II. p.510). Variat: b. typharum Desmaz. Op doode bladen van Typha latifolia. Vere. (Desmaz. Pl. Crypt. de Fr. no 1778) In Herb.: Zuid-Beveland, v. d. B. (v. s.)

312:. \$. herbarum Fr. $0 p$ doode stengels van groote kruiden. Hieme. Vere. (Fr. Syst. II. p. 511; R abenh. p. 174; Dz. et $\mathbb{H}$ b. Bijdr. I. p. 21). Variat: a. $0 \mathrm{p}$ Aster tripolium. In Herb.: Zuid-Beveland, v. d. B. (v. s.)

b. Op Solanum tuberosum. In Ilerb.: Zuid-Beveland, v. d. B. ; Leiden, W tt w.

c. Op Allium porrum. In Ilerb.: Maastricht, Franq. (v. s.)

d. Op Convolvulus tricolor. In Herb.: Zuid-Beveland, v. d. B. (v. s.)

e. Op Koolstronken. In Herb,: Amsterdam, v. d. S. Lac. ( $\nabla$. s.) 
f. Op Rumex. In Herb.: Amsterdam, v. d. S. L a c. (v. s.)

g. Op Centaurea en Carduus. In Herb.: Leiden, $\mathrm{W} t \mathrm{t}$ w. (v. s.)

h. Op Eryngium naritimum. In Herb.. Katwijk, $\mathrm{W} t \mathrm{tw}$. (v. v. et s.)

\$123. \$. Dardanae Wallr. Op doode stengels van Lappa tomentosa. Hieme. (W allr. Comp. fl. Germ. IV. pag. 806; R a ben h. p. 179)

In Herb.; Utrecht, v. d. S. Lac. (

31211. G. carduorum Wallr. $O p$ doode stengels van Carduussoorten. Hieme.

Pyreniis gregariis hypophloeodeo-latitantibus, basi arcte insculptis depresso-grlobosis, e vertice convexius. culo mox deplanato collum breve, apicem versus incrassatum obconicum truncatum pertusum, summam epidermidem penetrans informantibus. (Wallr. Comp. fl. Germ. IV. p. 805, excl. Syn. Sow. Fr.) In Ilerb.: Rijnsburg, de $\mathrm{Haan}$. (v. v. et s.)

3125. ฐ. armeriae $\mathrm{Corda}$. Aan affestorven stelen van Slatice globularifolia. Vere. (Corda' Ic. fung. IV. p. 41. Tab. VIII. fig. 119; R a ben h. p. 175)

In Herb.: Leiden, in den Hortus Academicus, $\mathrm{W} t \mathrm{tw}$. (v. s.)

3126, 9. peqforans Rob. Op doode bladen van Psamma arenaria. Vere.

Epiphylla, sparsa. Peritheeiis immersis, minutis, nigris ellipsoideis; ostiolis perforantibus, superficialibus, convexis, dein collabescendo subconcavis, poro dilatato apertis. Sporidiis ellipticis, hyalinis, bilocularibus. (Desmaz. Nouvelle notice crypt. inéd. de Fr. mars 1843. p. $41 \mathrm{n}^{\circ} .30$; Rob. in Desmaz. Pl. crypt. de $\mathrm{Fr}^{\mathrm{r}} \cdot \mathrm{n}^{\circ}$. 1288)

In IIerb.: Oreral in de Ilollandsche duinen. 
312\%. Ф. Merkeleyi Desmaz. $0 p$ doode stengels van Umbelliferae en vooral van IIeracleum spondylium. Hieme et Vere. (Desmaz. Pl. crypt. de Fr. no 1769 , sine descr.; Sph. Angelicae Berk. in Mag. of Zool. and Bot. vol. I. p. 48. Tab. 3. f. 7)

In Herb.: Zuid-Beveland, v. d. B. (v. vo et s.)

3128. Sph. nigrella $\mathrm{Fr}$. 0 p doode stengels van kruidgewassen. Vere. Aest. (Fr. Syst. II. p. 512; Rabenh. Herb. viv. myc. $\mathbf{n}^{\circ}$. 1936; Dz. et Mlb. Bijdr. III. p. 8)

In Herb.: Warmond, van Hall. - Bovendien: Maastricht, Franq. (v. s.)

3129. Iph. migrans $R o b .0 p$ doode halmen van Dactylis glomerata. Vere.

Ilinuta, sparsa, epidermide nigricante tecta. Peritheciis globosis vel subellipticis, depressis, albo farctis, basi villis brunneis vestitis. Ostiolo erumpente papillaeformi. Ascis clavalis; spuridiis fusiformibas curvulis, subhyalinis, 5 septatis (Des maz. 13e Not. pl. crypt. de Fr. 2e Série T. VI. p. 79. - Rob. in Desmaz. Pl, crypt. de Fr. $\mathrm{n}^{\circ} \cdot 1774$ )

In Herb.: Boxmeer, v. d. B. (v. s.)

3130. Sph. Iugubris $\mathrm{Rob}$. Op doode en rottende bladen van Psamma arenaria. Hieme. Veré. Aestate.

Haculis epiphyllis, piceis, minutis, ovalibus, sparsis. Peritheciis subsolitariis, immersis, nigris, globosis, demum depressis. Ostiolo brevi erumpente subconico. Ascis magnis cylindricis. Sporidiis 8, uniserialibus, ellipsoideis, subacuminatis, brunneis, semiopacis (Desmaz. 14e Not. pl. crypt. de Fr. no. 60. in Ann. des Sc. nat. 2 éér. Tom. VIII, p. 174; Rob. in Desmaz. Pl. crypt. de Fr. n०. 1792)

In Herb.: Overal in de Hollandsche duinen. ( $\vee$. v. et $s_{\text {s) }}$ 
Aanm. Deze soort zal eigenlijk wel in de hieronder volgende afdeeling der Foliicolae te huis behooren.

* 25. Perithecia cum matrice conereta, tecta, nulla macula exarida cincta (Foliicolae $\mathrm{Fr}$.)

3131. Siph. tubaeformis Tode. $0 p$ doode en afgevallen Elzenbladen. Vere. (Fr. Syst. II. p. 516; Rabenh. p. $172 ; D z$. et $M$ b. Bijdr. I. p. 21)

In Herb.: Warmond, de Ha a $\mathrm{n}$; Leiden, Voorschoten, Dz, et II b., Wttw., Ankers.; de Bildt, v. d. S. Lac. (v. vo et s.)

3132. Sph. gnomon $\mathrm{T}$ ode. $\mathrm{Op}$ afgevallen bladen van Corylus avellana. Hieme. Vere. (F r. Syst. II. p. 517; Rabenh. p. 172)

Maastricht, Franq.

3133. Sph. Saligna Ehrh. Op doode bladen van Salix capraea. Hieme. Vere. (Sphaeria capraea Dec.; Fr. Syst. II. p. 517; Rabenh. p. 172 ; Phoma saligna Fr. Syst. II. pag. 546; Rabenh. p. 143)

In Herb.: Naastricht, Franq. (v. s.)

3131. Sph. setacea Pers. Op doode en afgevallen bladen van verscheiden boomen. Hieme et Vere. (Fr. Syst. II. p. 518; Rabenh. p. 173). Variat:

1. petiolicola West. $\mathrm{O}_{\mathrm{P}}$ de rottende bladstelen van Acer pseudo-platanus.

In Herb.: Amsterdam, v. d. S. L a c. (v. v. et s.)

3135. Sph. aquilina Fr.? $0 p$ de bladen van Pteris aquilina. Ilieme. (F r. Syst. II. p, 522; R abenh. p. 169)

In Herb.: Utrecht, Jungh. (v, s.)

3136. Eph. polypodili Rabenh. $O_{p}$ de bladen van Polypodium vulgare. Aest. (Sph.aquilina Fr. in Dz. et II b. Bïjdr. III. p. 8)

Peritheciis sparsis, depresso-globosis, opacis, subumbonatis; ascis cylindraceo-clavatis octonis oblongis 1/420"' crassis 3-4 plo longioribus hyalinis proto- 
plasmate turbatis dense farctis. (R a be $\mathrm{h}$ h. Herb, vir. myc. 2e Edit. n. 533)

In Herb.: Leiden, D za et II b.; Zuid-Beveland, v. d. B. (v. s.)

3138. Gph, carpinea Fr. $0 p$ doode blacien van Carpinus betulus. Hieme et Vere. (Fr. Syst. II. p. 523; Dz. et 1 iI b. Bïjdr. III. p. 8)

In Herb.: Utrecbt, v. d. S. Lac. (v. s.)

3135. פph. myriadea Dec. $O_{p}$ doode en afgevalion Eikenbladen. Vere. (Fr. Syst. II. p. 524; Rabenh. p. 188)

In Herb.: Zwake, v. d. B. (v. s.)

3139, 5ph. brunneola $\mathrm{Fr}, O_{p}$ kwịnnende of afgestorven bladen van Convallaria majalis. Aut. Hieme. (Fr. Syst. II. P. 526; Rabenh. p. 580)

Haastricht, Franq.

3140. Sph. maculaeformis Pers. Op doode Eiken- en Kastanjebladen, Ilieme et Vere. (Fr. Syst. II. p. 524; Rabenh. p. 168; Dz. et II b. Bijdr. I. p. 21)

In Herb.: Leiden, Dz. et M b.; Utrecht, Dz. et H b.; Kampen, R. B ond.; Maastricht, Fran q. (v. s.)

3141. Sph. aucupariae Lasch. $O_{p}$ levende blacen van Sorbus aucuparia. Acst. (Raben h. pag. 170 ; Sph. conglomerata (Sorhi) Wallr. Comp. Fl. Germ. IV. p. 814)

In Herb.: Domburg, v. d. B.; Mastricht, Franq. 3142. Sph. punetiformis Tode. Op doode Eikenbladen. Hieme et Vere. (Fr. Syst. II. p. 525; R a benh. p. $168 ;$ Dz, et II h. Bijdr. I. p. 21)

In Herb.: Leiden, $D_{z}$. et $M \mathrm{~b}$.; Zwake, v. d. B. (v. v. et s.)

3143. Sph. eryngii $\mathrm{Fr} . \quad O_{p}$ donde bladen en hladstelen van Eryngiun maritimum. Vere. (Fr. in litl. Duby Bot. Gall. II. p. 710 ; Rabenh. p. 171) Va:iat: 
a. petiolicola West. In Herb.: Katwijk, de Il a an, $\mathrm{W} t \mathrm{t}$.

3144. Sph. cruciferarum Fr.' $0 p$ de stengels, stelen en zaadhuisjes van Erysimum officinale. Aut. Hieme. (Fr. Syst. II. p. 525)

In Herb.: Zuid-Beveland (Goes), v. d. B. (v. s.)

3145. Sph. ostruthii Fr. Op de stervende bladen van Angelica sylvestris. Aut. (Fr. Syst. II. p. 526; Depazea Ostruthii Rabenh. p. 139)

In Herb.: Zwijndrecht, v. d. S. L a c. (v. v. et s)

3146. Sph. atomus $D$ esmaz. $O p$ doode Beukenbladen. Aut.

Epiphylla; maculis rofis, minutis, suborbiculatis; peritheciis minutissimis, numerosis, brunneis, innatoprominulis, collabescendo umbilicatis (Desmaz. $8^{\mathrm{e}}$ Not. crypt. nouv. de France $n^{\circ} .19$, in Ann, des Sc. nat. IIars 1841; Desmaz. Pl. cr. de Fr. $n^{\circ}$. 1293)

In Herb.: Leiden, Dz. et MI b.; Zuid-Beveland, v. d. B. (v. v. et s.)

3148. Sph. melanoplaca Desmaz. Op treurende bladen van Geum urbanum. Aut.

Amphigena. Peritheciis minutissimis, numerosis innato-prominulis, nigris, subnitidis, poro simplici pertusis, in macula magna fuliginea gregariis. Nucleo albido. Ascis brevibus cylindricis, crassis, absque paraphysibus; sporidiis glancis vix distinctis (Desmaz. 20 Not. crypt. nouv. de Fr. $n^{\circ} \cdot 10$, in Ann. des Sc. nat. 2⿺̀ Sér. Tom. XVIII. - Desmaz. PI. crypt. de Fr. $n^{\circ}$. 2097)

In Herb.: Maastricht, Franq. (v: v. et s.)

3145. Sph. gangrena $\mathrm{Fr}$. $0 \mathrm{p}$ treurende bladen van verschillende Grassoorten. Aut.

Tectá inaequalis aterrima receptaculis astomis pro- 
minulis demum depressis tuberculosa (Fr. in Duby Bot. Gall. II. p. 695).

Pour compléter cette description, nous dirons que les perithèces sont hypophylles, d'abord convexes, ensuite affaisés et concaves, munis d'un ostiole ponctiforme. Les thèques sont claviformes grosses, remplies de sporidies oblongues, obtuses, 3 à 4 fois plus longues que larges, contenant trois à quatre sporules globuleuses et hyalines (Des maz. Pl. crypt. de Fr. $\left.n^{\circ} .1267\right)$

In Herb.: Boxmeer, v. d. B. (v. s.)

\section{Trib, Dothidini.}

POLYפTIGMA Per 8. in Ho ug.

3149. P. fulvum Pers. $0 p$ de levende bladen vats Prunus spinosa. Aut. (Rabenh: I. p. 166; Dothidea fulva Fr. Syst. II. p. 554; D z. et M b. Bijdr. I. p. 22)

In Herb.: In 't Haagsche Bosch, Dz. et $\mathrm{II} b$. ( $v$, v. et 3.). Variat:

a. aurantiacum West. $\mathrm{Op}$ Perenbladen. Aest. (Polyst. aurantiacum West. Ilerb. crypt. Belgge $\left.n^{\circ} .825\right)$

In Herb.: liotterdam, O u dem.; Kampen, R. B on d. ; Zuid-Beveland (Goes), vi d. B. (v. v. et s.)

3150. P. rubrum Dec. Op levende bladen van Prunus spinosa. Aut. (Rabenh. I. p. 166; Dothidea rubra Fr. Syst. II. p. 553; Dz; et II b. Bijdr. I. p. 22)

In Herb.: Amsterdam, D z. et $\mathrm{Ml}$ b. - Bovendien: Maastricht, Franq. (v. v. et s.)

3151. P. typhinum $\mathrm{D}$ ec, $0 \mathrm{p}$ de halmen van Agrostis en andere Grasscorten. Aut. (Rabenh. I. p. 167; Do- 
thidea typhina Fr. Syst. II, p. 553; Dz. et Mb. Bijdr. I. p. 21)

In Herb.: Amsterdam, v. d. S. La c.; Haarlemmerhout, M. Doornik; Zuid-Beveland, v. d B. - Bovendien: Maastricht, Franq. (vo vo et s.)

\section{DOTHIDER Fries.}

3152. D. ribesia Fr. Op doode Aalbezietronken en -takken. Aut. et Vere. (Fr. Syst. IL. p. 550; Rabenh. p. 164)

In Ilerb.: Zuid-Beveland (Goes), v, d. B. - Bo* vendien: Mlastricht, Franq. (v. v. et s.)

3153. H. sambuci Fr. Op doode Vliertakken. Hieme et Vere. (Fr. Syst. II. p. 151; R ab enh. p. 164)

In Herb.: Leiden, Dz, et If b. (Sub nomine Sph. pulicaris). Muastricht, Franq. (v. s.)

3耳直. puceinioldes $\mathrm{Fr}$. Op doode takken van Buxus sempervirens. Hieme et Vere. (Fr. Syst. II. p. 551; Rabenh. p. 164)

In Herb.: Zuid-Beveland (Goes), vo d. B. (v. so)

3155. ID. \$pharoides Fr.? Op de schors van doode Esschelakken. Hieme et Vere. (Fr. Syst. II. p. 552; Rabenh. p. 165)

In Herb.: Amsterdam, v. d. S. Lac. (v. s.)

3156. Dernicosa $\mathrm{Fr}$. $\mathrm{O} p$ doode stengels ran Anthriscus sylvestris. Aut, Hieme. (Fr. Syst. Il. p. 559; Rabenh. p. 165)

IIIastricht, Franq. (n. vo)

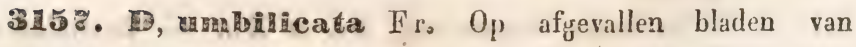
Crataegus oxyacantia. Aut. Hieme. (Fr. Syst. Orb. veg. I. p. 363 ; R a benh. P, 165)

Naastricht, Franq. (n. v.) 
3158. alnea Fr. Op stervende Elzenbladen. Aut. (F r. Syst. II. p. $564 ; \mathrm{Dz}$. et $\mathrm{M}$ b. Bijdr. II. p. 17 ; Sphaeria alnea Link; Rabenh. p. 170)

In Ilerb.: Oosterhoutsch bosch te Ubbergen, A bel.; Leiden, Dz. et $\mathbf{M l b}$. - Bovendien: Maastricht, Fran q. (v. v. et s.)

3159. Potentillae $\mathrm{Fr} .0_{\mathrm{p}}$ levende bladen van $\boldsymbol{P}_{0}$. tentilla anserina. Aut. (Fr. Syst. II. p. 563; R abenh.p. 165; Dz. et II b. Bijdr. IlI. p. 8)

Maastricht, Franq. (n. v.)

3160. ID. Drassicae Desmaz, $O p$ treurende Koolbladen. Aut.

Amphigena; maculis orbiculatis, tandem confluentibus, pallide viridibus vel griseis subzonatis, centro rufescentibus subexaridis; peritheciis minutissimis, numerosis, prominulis, nigris, aggregatis, subconcentricis; ascis fixis, subclavatis; sporidiis oblongis; sporulis 2-4, globosis, hyalinis (Desmaz, 9e Not. crypt. nouv. de Fr. in Ann. des Sc. nat. Févr. 1842. Desmaz. Pl. crypt. de Fr. Nouv. Edit. no ${ }^{0}$ 95)

In Herb.: Leiden, $\mathrm{D}_{\mathrm{z}}$. et $\mathrm{H}$ b.; Utrecht, $\mathrm{W} t \mathrm{t} \mathrm{w}_{\text {。 }}$; Zuid-Beveland, v. d. B. (v. v. et s.)

3161. H. chaetomium $\mathrm{Kz}$ e. Op treurende bladen van verschillende Rubus-soorten. Aut. (Fr. Syst. II. p. 565; Status vetustis $=$ Phacidium rubi Fr. Syst. II. p. 578; Rabenh. p. 158)

In Herb.: Zuid-Beveland, v. d. B. (v. s.)

3162. D. decolorans $\mathrm{Fr}$. $0 p$ doode lakken van Morus alba. Hieme et Vere. (Fr. Elench. II. p. 122; Desmaz. Pl. crypt. de Fr. Nouv. Ed. no. 90)

In Herb.: Zuid-Beveland, v. d. B. (

3163. ID. fimbriata $\mathrm{Kickx}, \mathrm{Op}_{\mathrm{p}}$ levende bladen van Carpinus betulus. Aut. (K ickx Fl, crypt. Louv. p. 
112. Sphæria fimbriata Pers.; Fr. Syst. II. p. 436; Rabenh. p. 189)

In Herbs: Rotterdam, 0 u dem. (v. v. et s.) - Bovendien: Maastricht, Fran q.

3164. ID, fraxini $\mathrm{Fr}_{0}, 0 p$ de bladen van Fraxinus excelsior. Aut. ( $\mathrm{Fr}$. Syst. II. p. 561; D z. et II b. Bijdr. I. p. 22)

In Herb.: Leiden, Dz. et M b.

3165. D. geographica Fr. $0 p$ doode en afgevallen Appelbladen. Hieme. Vere. (F r. Syst. II. p. 560; Sphaeria geographica Wallr.; Rabenh. p. 188)

In Herb.: Amsterdam, v. d. S. Lac. (v. v. et s.)

3166. D. graminis $\mathrm{Ch}$ ev. Op de bladen van Deschampsia caespitosa en andere Grassoorten. Aut. Hieme.

Subrotunda prominula nigra ragulosa striata, stromate atro, nucleis minutis albo-farctis seriatisque. (Chev. Fl. Par. I p. 457. Non Sphaeria graminis Pers.)

In Herb.: Leiden, A nkersm., Dz. et II b.; Mlaas. tricht, Fr anq.; Zuid-Beveland, v. d. B.

316\%. D. depazoldes $\mathrm{Desmaz}$. Op levende bladen van Buxus sempervirens. Per annum.

Epiphylla. Maculis eburneis, nigro-cinctis. Pustulis atris, orbiculatis, convexiusculis, sparsis, dein confluens angulato-difformis. Nucleis 3-20, confertis, minutissimis, albis, ascis clavatis brevibus. Sporidiis oblongis, hyalinis. Sporulis 4, globosis. (Desmaz. Crypt. inéd. de la Fl. de Fr. n. 20 in Mém. Soc. Roy. des Sc. de Lille 1839. $1^{\text {re }}$ partie pag. 85 )

In Herbs: Maastricht, Franq. (v. s.)

3169. D. iridis Desmaz. $0 p$ de stengels en bladen van Iris pseudo-acorus. Aut.

Maculis rufis rel brunneo-castaneis, Peritheciis 
minutissimis, sparsis, nigris, nitidis, innato-prominulis, convexis, intus albidis, poro pertusis, demum depressis. Ascis majusculis subcylindricis; sporidiis oblongis, utrinque obtusis, hyalinis 2, 3 septatis (Desmaz. $14^{\mathrm{e}}$ Not. crypt. inéd. de Fr. $\mathrm{n}^{0} .65$, in Ann. des Sc. nat. $2^{\text {e }}$ Sér. T. VIII. p. 176)

In Herb.: Leiden, $\mathrm{Dz}$. et $\mathrm{M}$ b. (v. v. et 8. )

3169. D. robertiani Fr. Op levende bladen van Geranium Robertianum. Aut. (Fr. Syst. II. p. 564; Sphaeria robertiani Kabenh. p. $169 ; \mathrm{Dz}$. et $\mathrm{Ml}$ b. Bijdr. I. p. 22)

In Herb.: Amsterdam, $\mathrm{O}$ udem. - Bovendien: Maastricht, Franq. (v。 vo et s.)

3180. ID. ulmi Fr, $O_{p}$ levende en treurende Olmbladen. Aut. (Fr. Syst. II. p. 555; Polystigma ulmi Link.; R abenh. p. 167; Dz. et MIb. Bijdr. I. p. 22)

In llerb.: Zuid-Beveland, v. d. B.; Ubbergen bij Nijmegen, A b e l.; Kampen, R. B o n d. - Bovendien : Maastricht, Franq.

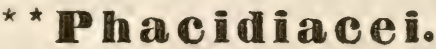

\section{Trib. Stegillacei.}

\section{EUST时 F F .}

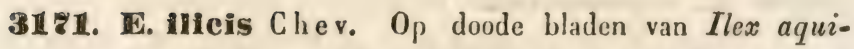
folium. Hieme. Vere. (Che v. Fl. Par. I. p. $443 ; \mathrm{Fr}$. Elench. II. p. 112; Stegilla ilicis Rabenh.p. 163; Dz. et $\mathrm{Ml}$ b. Bijdr. I. p. 19)

In Herb.: Leiden, Dz. et II b. 


\section{Trib. Hysteriacei.}

\section{IR IIITHSMI.}

3182. HB. urticae Fr. Aan doode stengels van Urtica dioica. Hieme. Vere. (F ғ. Syst. II. p. 570; R abenh. p. 161)

In Herb.: Haastricht, Franq. (v. s.)

3183. He. punctatum Fr. $O_{p}$ levende bladen van Acer pseudo-platanus. Aut. (Fr. Sysi. II. p. 569; Ra. benh. p. 161; Dz. et $\mathrm{Ml}$ b. Bijdr. I. p. 19)

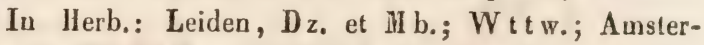
dam, v. d. S. Lac. (v. v. et s.)

3181. Mt. acerinum Fr. $O_{p}$ dezelfde bladen als de voorgaande. Aut. (Fr. Syst. II. p. 569; Rabenh. p. 161 ; Dz. et II b. Bijdr. I. p. 19)

In Herb.: Leiden, Dz. et $\mathrm{Nl}$ b.; Rotterdam, O udem.; Ubbergen bij Nijmegen, Abel.; Zwolle, R. Bond.; Zuid-Beveland, v. d. B. - Bovendien: Maastricht, Franq. (v. v. et s.)

3185. AR. Salicinum Fr. Op levende bladen van Salix capraea. Aut. (F r. Syst. II. p. 568 excl. var. $\beta$.; Rabenh. p. 162; Dz. et II b. Bijdr. I. p. 18)

In Herbı: Leiden, D z. et H b.; Valkenswaard, de II a a $\mathbf{n}$; Zuid-Beveland, v. d. B. - Bovendien: Haastricht, Franq. (v. v. et s.)

3186. PR. umbonatum Desinaz. Op levende bladen van Salix monandra. Aut. (Desmaz. Pl. crypt. de Fr. $n^{\circ}$. 1736 ; Rabenh. p. 162; Rh. salicinum $\beta$. minus Fr. Syst. II. p. 568; Dz, et M b. Bijdr. I. p. 18)

In Herb.: Leiden, Dz, et $\mathrm{Hl}$ b. (v. v. et s.)

318\%. HR. Bistortae $\mathrm{L}$ ib. $\mathrm{Op}_{\mathrm{p}}$ stervende bladen van $\boldsymbol{P}_{\mathrm{O}}$ lygonum bistorte. Aut. (Lib. Crypt. Ard, n 68 ; 
Ectostroma Fr. Syst. II. p. 602; Polystigma Liuk; Rabenh. p. 167)

Maastricht: Franq. (n. v.)

3188. He. rubiae Mont. $O_{p}$ de bladen van Rubia tinctorum. Aut.

Iunatum, hypophyllum, gुregarium confluensque, punctiforme, convexum, cupulari-depressum, fuscoatrnm, opacum, intus fuscum, frustulatim secedens: ascis e fundo erectis clavatis sporas 8 oblongas altero fine altenuatas quadrinucleolatas foventibus (ll on t. Syl. gen. spec. Crypt. p. 194)

In Herb.: Zuid-Beveland, v. d. B. (v. s.)

\section{HPCHBIU⿴囗十 $\mathrm{Fr}$.}

3199. P. coronatum Fr. $\mathrm{O}_{p}$ rottende Eikenbladen in vochtige bosschen. Hieme et Vere. (Tir. Syst. II. p. 577; Rabenh. p. 158)

Milaastricht, Franq. (n. v.)

3180. Mentatum $\mathrm{S} c \mathrm{~h}$. O O $\mathrm{O}_{\mathrm{p}}$ doode Eikenbladen. Hieme et Vere. (Fr. Syst. II. p. 577; Rabenh. p. 158)

Iu Herb.: Leiden, Dz. et li b.; Haastricht, Franq. (v. v. et s.)

3181. Pini Fr. Op doode en afgevallen takken van Pijnen en Denneu. Aut. Vere. (F r. Syst. II. p. 573; R abenh. p. 159)

In Ilerb.: Zuid-Beveland, v. d. B. (v. v. et s.)

3182. Hicis Lib. Op doode en afgevallen bladen vau Ilex aquifolium. Hieme et Vere. (Phacidium multivalve (ex parte) 11 oug. et Nestl.; Fr. Syst. 1I. p. 576; Ph. aquilolii habenh.p. 159; Ph. mullivalve Sch m.; Dz. et II b. Bijdr. I. p. 19)

$$
\text { In lierb.: Leiden, Dz, et II b. (v. v. et 8.) }
$$

3183. Ratella Pers, $O_{p}$ doode stengels van groote 
kruidgewassen. Aut. Vere. (Fr. Elench. II. p. 134 ; Rabenh. p. 160; Sphaeria patella Fr. Syst. II. p. 511). Variat:

a. campestre Fr. 1. 1. $0_{\mathrm{p}}$ de, stengels van Daucus carota.

In Herb.: Leiden, W $\mathbf{t}$ w.; Rijnsburg, d e II a a n; Maastricht, Fran q.; Utrecht, v. d. S. Lac.; ZuidBeveland, v. d. B. (v. vo et so)

B. alpestre Fr. I. I. $\mathrm{Op}$ de stengels van Angelica sylvestris.

Maastricht, Franq. (n. v.)

3151. P. lauro-cerasi $D$ esmaz, $O p$ doode bladen van Prunus lauro-cerasus. Hieme et Vere. (Fr. Elench. II. p. 136; D z. et II b. Bijdr. II. p. 19)

In Herb.: Zuid-Beveland, v. d. B. (v. s.)

3155. P. ranunculi $\mathrm{Fr} \cdot \mathrm{O}_{p}$ stervende bladen van $R a$ nunculus acris en repens. Aut. (Lib. Pl. cr. Ard. $\mathrm{n}^{\circ}$. 69 ; D z. et MI h. Bijdr. II. p. 16; Dothidea ranunculi Fr. Syst. II. p. 562; Excipula rananculi $\mathrm{Ra}$ benh. p. 153)

In Herb.: Naaldwijk, vo d. Tr.; Zuid-Beveland, v. d. B. (v. s.)

3186. Pr. litigiosum $\mathrm{D}$ es maz. Op treurende bladen van Ranunculus acris. Aest. et Aut.

HIaculis amphigenis, irregularibus, luteolis. Peritheciis hypophyllis, gregariis, minutis, rufo-brunneis. Disco fulro-luteo, plano vel convexiusculo, margine sinuoso. Ascis clavatis sporidiis oblongis subpyriformibus. (Desmaz. 14e Not. crypt. nouv. de Fr. $n^{\circ} .74$ in Ann. des Sc. nat. $2^{\circ}$ Série, T. VIII, p. 181 ; Desmaz. Pl. crypt. de Fr. $n^{\circ} .1639$ )

In Herb.: Zuid-Beveland, v. d. B. (v. s.)

318\%. abietis $R$ abenh. Op de doode schors en takken van Pinus abies. Aut. (Rabenh. p. 161) In Ilerb.: Leiden, W $t \mathrm{t}$ w. ex herb. Jungh. (, $\left.\mathrm{S}_{\text {. }}\right)$ 
IIYSTERIUMI ( $\mathrm{T}$ ode) $\mathrm{F} r$.

A. enudata Fr. (III sterium Dec.)

3188. IL. pulicare Pers. Op roltend hout en schors van oude Populieren en Eiken. Per annum. (Fr. Syst. II. p. 579; R abenh. p. 153; Dz. et II b. Bijdr. III.p. 7)

In Herb.: Harderwijk, Jungh., W $t \mathrm{t} w . ;$ 's Gravenhage, Stekh.; Leiden, W $t \mathrm{tw}$. - Bovendien: Maastricht, Franq. (v. v. et s.)

3159. H. grammodes de Not. Op rottend hout en oude Eikenplanken en -palen. Hieme et Vere.

Nigro-piceum nitidum, sparsum, sporis latioribus. (de Not. Dispos. in Giorn. bot. Ital. T. JI. $a^{\circ} 2^{\circ} \mathrm{p}$. 21. - Hyst. Rousselii $\beta$. Grammodes Duby Mlém. sur la tribu des Hystérinées p. $28 \mathrm{n}^{\circ}$. 13)

In Herb.: Utrecht, v. d. S. Lac. (v. s.)

3199. H. Lineare Fr. Op rottend hout in holle Wilgen. Hieme et Vere. (Fr. Syst. II. p; 583; Rabenh. p. 154.)

In Herb.: Willemsdorp, Goes, $\mathbf{0}$ d. B, (v. 8.)

\section{酯. r m mentia.}

3191. 11. fraxini Pers. Op schors en takken van Esschen. Aut. Vere. (Fr. Syst. II. p. 585; Rabenh. p. 155; Dz. et $\mathrm{Ml}$ b., Bijdr. I. p. 19)

In Herb.: Leiden, Stekh., Dz. et Hb., $O$ udem., W ttw. ex herb. Ankers.; Utrecht, v. d. S. Lac.; Maastricht, Franq. (v. v. et s.)

3192. H. rocheanum Duby. Op een onbekenden bast.

Receptaculis erumpentibus superficiali-adnatis crustaceo-aggregatis sed non confluentibus ovalo-globosis ovoideisve atro-nitidis, labiis tumidis convexis cstriatis rimam angustan deınum paulisper dilatalam relinquen- 
tibus, thecis clavatis sessilibus sporas diametro subduplo longiores ellipticas tenuissime lineolatas diaphanas maturitate pallide luteolas nacleis 4 merenchymaticis faetas foventibus, paraphysibus filiformibus apice ramosis et interdum coadunatis breviorihus. (D u b y Mém. sur la trib. des IIyst, pag. 31. $\mathrm{n}^{\circ}$. 23. Tab. I. fig. 11)

Leiden, in den Hortus academicus, de la Roche in IIerb. Dec. (n. v.)

3193. HII. rugosum Fr. Op levende boomschors. Per annum. (Fr. Elench. II. p. 141). Variat:

a. faginea Fr. I. 1. p. 14́3. Op Beukenschors. (Opegrapha conglomerata Pers.; Rabenh. p. 155)

b. quercinum Fr. I. I. $O_{p}$ de schors van jonge Eiken. (Opegrapha macularis Ach.)

In llerb.: Twello, R. Bond., Top (v. v. et s.)

3194. H. conigenum Moug. et Nestl. Op rottende kegelschubhen van Pinus sylvestris. Hieme et Vere. (Fr. Syst. II. p. 586; Rabenh. p. 155)

In Herb.: Leiden, Stekh., W $t \mathrm{tw}$. Bovendien: Maastricht, Franq. (v. s)

\section{Subtecta Fr.}

31D5. HI. rubi Pers. Op afogestorven ranken van Rubussoorten. Aut. Vere. (Fr. Syst. II. p. 587; Rabenh. p. 155)

In llerb.: Utrecht, Witw. ex herb. Jungh ; Maastricht, Franq. (v. v.)

3196. II. pinastri Schrad. $O_{p}$ afgevallen en rottende Pịn- en Dennenaalden. Hieme et Vere. (Fr. Syst. II. p. 587; Rabenh. p. 156; Dz. et II b. Bijdr. I. p. 19)

In Herb.: Harderwijk, Jungh.; Leiden, Stekh.; Dz. et II b. (v. vo et 3.). Variat: 
a. major West. Op de naalden van Pinus maritima. (West. 6e Not. crypt. inéd, de la Belg. $\mathrm{n}^{\circ} .68$ in Bull. Acad. Roy. des Sc. de Belg. 2e Sér. T. VII)

In Herb.: Bij Zutphen, West. ex herb. G. $\Lambda$ ubert (v: v.)

b. minor West. I. I. Op de naalden van Pinus Zembra.

In Herb.: Bị Zutphen, West. ex herb. G. A ubert (v. s.)

c. juniperinum $\mathrm{Fr}$. $0 p$ de naalden van Juniperus sabina en communis (Fr. Syst. II. p. $588 ; \mathrm{D}$ z. et M b. Bịdr. I. p. 19)

In Herb.: Leiren, Dz. et Il b.; bij de Bildt, v. d. S. L a c. (v. v. et s.)

319\%. III. Iauri Fr. $O_{p}$ doode en stervende bladen van Laurus nobilis. Per annum (R a benh. I. p. 156. IIypoderma lauri Duby. Mém. de la trib. des Ilyst. p. 43)

In Herb.: Leiden in den. Horlus academicus, Witw. (v. s.)

\section{E. $\mathrm{y} 1 \mathrm{om} \mathrm{mr}$.}

3198. H. scirpinum $\mathrm{Fr} . \quad 0 p$ rottende halmen van Scirpus lacustris, Aut. llieme. (F r. -Syst. II. p. 590; R abenh. p. 157)

In llerb.: Leiden, Wttw, ex herb. Jungh. (v. v. et s.)

3199. HI. a rundinaceum $\mathrm{Sch} r$ ad. $O p$ doode halmen van Phragmites communis. Ilieme et Vere. (Fr. Syst. II. p. 590; Rabenh. p. 157)

In Herb, : Leiden, Dz, et MIbo, vo d. B.; Utrecht, v. d. S. Lac, W $\mathrm{ttw}$, ex herb. Jangh,; Zuid-Beveland, v, d. B. (v, v. et s.) 
320๑. It. culmigenum $\mathrm{Fr} .0_{p}$ doode halmen van verschillende Grassoorten. Hieme. (Fr. Syst. II. p. 591; Rabenh. p. 157). Variat:

a. gramineum Fr. I. l. $0_{p}$ de halmen van Koorn, enz.

In Herb.: Zuid-Beveland (Goes), v. d. B. (v. s.)

b. abbreviatum Desmaz. Op doode bladen van Psamma arundinacea (Desmaz. 14e Not. in Ann. des Sc. nat. 2 e Série T. VIII. p. 179; Desmaz. Pl. crypt. de Fr. Nouv. Ed. n. 171)

In Herb.: Ilollandsche Duinen, O adem. (v. vo et s.)

3201. If. foliicolum $\mathrm{Fr}$. $\mathrm{O}_{\mathrm{p}}$ de doode bladen van $\mathrm{Cra}$. taegus oxyacantha. Aut. Ilieme. (Fr. Syst. II. p. 592 ; Rabenh. p. 157)

In Herb.: Nymegen, A bel. (v. v. et s.)

3202. III. hederae Mart. $O p$ de doode bladen van Hedera helix. Aut. Vere. (Fr. Syst. II. p. 593; Rabenh. p. 156)

In Herb.: Leiden, Stekh. (v. v. et 8.)

3203. II. punctiforme $\mathrm{Fr}$. $O p$ stervende Eikenbladen. Aut. Hieme. (Fr. Syst. II. p. 593; R abenh. p. 157; Hyster. virgultorum v. quercinum West. Herb. crypt. Belge $n^{\circ}$. 1119)

In Herb.: Haarlem, v. d. B. (v. s.)

\section{A YLDERAPHUTI Lib.}

Perithecium elongatum, dimidiatum, innatum simplex ramosumve, rima longitudinali dehiscens, nucleo discifero pellucido albido lineari persistente; asci minuti fixi paraphysibus immixti; sporidiis simplicibus. (Lib. Pl. crypt. Ard, nn. 272)

3204. A. vagans Desmaz. Op de bladen van Ilex aquifolium, Hedera helix, etc. Hieme.

Peritheciis innato-superficialibus, amphigenis, sparsis 
ovato-oblongis, simplicibus vel confluente furcatis, atris; labiis subcristatis. Ascis ellipsoideis; sporidiis oblongis, obtusiusculis; sporulis 4, globosis, opacis. (Desmaz. Not. sur Cr. inéd. de la Fr. in Mém. de la Soc. Roy. des Sc. de Lille, Mar's 1843. $n^{\circ} .39$; A. Hederae Lib. 1. I. $n^{\circ}, 272$ ).

In Herb.: Busloo bij Zutphen, West, ex herb. G. Aubert. (v. v. et s.)

\section{COL HIII $\mathrm{W}$ a $1 \mathrm{r}$.}

Receptaculum simplex coriaceo-spongiosum flexuosum ellipticum aut lineare basi applanata adnatum primo clausum dein rima longitudinali excurrente bilabiatodehiscens, tandem labiis divergentibus omnino apertum, senio collabescens canaliculatum. Nucleus gelatiniformis albescens. Thecae elongato-clavatae e sporis 8 filiformihus pallide hyalino-lutescentibus formatis quae apice disjunguntur, inflectuntur, et basi fixae speciem paraphysium filiformium induunt. (Wallr. char, reform. e el. Duby. mém. sar la tribu des Hyst.p. 50)

3205. C. quercinum Wallro $0 p$ doode tahken en takjes van Eiken. Aut. Vere. (Cenangium quercinum Fr. Syst. 11. p. 189 ; Dz. et Mb. Bijdr. II.p. 16; Hysterium quercinum Pers.; Rabenh. p. 155; exel. Syn. Fr. El.) In Herh.: Leiden, $\mathbf{D} z$, et $\mathbb{M}$ b.; bij den Uilenpas, II. J.; Haastricht, Franq. (vo vo et s.)

\section{EXCRULA $\mathrm{Fr}$.}

3206. E. Heraclei $R$ abenh. Op treurende bladen van Heracleum spondylium. Aut. (R abenh. p. 152; Dothidea heraclei Fr. Syst. II. p. 556). In Herb.: Amsterdam, v. d. S. La c. (v. s.)

3208. E. rubi Fr. Op afgestorven ranken vais verschilw 
lende Rubus-soorten. Aut. Vere. (F r. Syst. II. pag 190; Rabenh. p. 152).

In Herb.: Zuid-Beveland, v. d. B. (v. vo et s.)

\section{** Sphaeronemei.}

\section{Trib. Cytisporei.}

\section{HIEN DEIBSONHA Berk.}

Perithecium corneum, superficiale innatum, vel immersum, vertice poro perforatum vel irregulariter apertum, vel ostiolatum; ostiolo punctiformi, plus minusve cylindrico. Asci nulli; basidia filiformia vel stipitiformia plus minusve elongata aut brevissima. Sporidiis terminalibus, polymorphis, dein liberis; sporulis plus minusve numerosis, globosis, cylindricis vel discoideis, achromaticis aut coloratis. (Berk. emend. Desmaz. 17 e Not. crypt. nouv. de $\mathrm{Fr}$ in 'Ann: des Sc. nat. 2. Série Tom. XI, Mlai et Juin 1849, p. 15)

3208. 职. phragmitidis $\mathrm{D}$ es $\mathrm{maz}, \mathrm{O}_{\mathrm{p}}$ de rottende bladscheeden van Arundo phragmites. Hieme et Vere.

Peritheciis innatis, minutissimis, numerosis, sparsis, globosis, nigris, epidermide tectis. Ostiolis erumpentibus papillatis halone brunnea cinctis, demum deciduis. Sporidiis ovoideo-oblongis, sporulis 4, compressis, fuscis. (Desmaz. 22 Not. crypt. nouv, de Fr. in Ann. des Sc. nat. $2^{e}$ Sér. Tom. XX, n 19).

In Herb.: Amsterdam, v. d. S. Lac. (v. s.)

3209. II. mespili West. Op stervende bladen van Mespilus germanica. Aut.

Taches épiphylles, éparses ou confluentes, arrondies ou irrégulières, brunes, blanchissant au centre avce l'âze. Périthèces raves, très, petits, noirs, eparpillés 
irrégulièrement sur toute la tache. Sporidies hyalines, ovale-pyriformes, à trois cloisons et mesuránt $3 / 200^{\circ}$ de mill. de longueur sur $1 / 150_{e}$ de mill. de larreur. (W es f́. 5e Not. crypt. inéd. de la fl. Belg. in Bull. de l'Acad. Roy. des Sc. de Belg. 2e Série T. II, parg. 16, n 14 ; West. Herb. Cr. B. $n^{\circ}$ 1225)

In Herb.: Utrecht, v. d. S. Lac. (v. s.)

3210. HI. Fiedleri West. Op afgestorven takken en takjes van Cornus sanguinea en alba. Aut. Vere.,

Sporis ellipticis, oblongis, triseptatis; septis laete castaneis. (Klotzsch in Rabenh. Herb, viv. myc. $1^{2}$ ed. $n^{\circ} 882 ; 2^{a}$ ed. $\left.n^{\circ} 71\right)$

In Herb.: Utrecht, vo d. S. La c. (v. v. et s.)

3211. H. sarmentorum West. $O_{p}$ doode ranken van Vitis vinifera. Hieme.

Périthèces immergés, aplatis, d'un brun foncé, cam chés sous l'épiderme du sarment qui se déchire en plusieurs lambeaux, pour faire voir un ostiole poriforme. Basidies simples, continues, transparentes, restant attachés aux sporidies pendant leur irruption. Sporidies brunâtres, pyriformes, à trois cloisons: (West. 2e Not. crypt. inéd. de la Belg, in Bull. de l'Acad. Roy. des Sc. de Belgique, T. VIII, p. 14, $\mathrm{n}^{\circ} 60$ fig. 2).

In Herb.: Leiden, Stekh. (

3212. 1I. subseriata Desmaz. $O p$ doode halmen van grassoorten en hijzonder van Enodium caeruleum. Vere.

Erumpens, peritheciis nigris, subseriatis, minutissimis globosis vel oblongis. Nucleo albido, dein griseo. Sporidiis fusiformibus, $3-6$ septatis, sporulis $4-7$ glohosis, hyalinis. (Desmaz. 13e Not. erypt: inéd, de Fr. pag. 69. $\mathrm{n}^{\circ} 13$; Pl. crypt. de Fr, $\mathrm{n}^{\circ}$ 1893).

In IIerb.: Zuid-Beveland, v. d. B. (v. v. el so) 


\section{PESTARTL Denot.}

Sporidia pedicellata plariseptata, articulo supremo in setas divergentes soluto, infero mutico, vel uni-bi-se10so, stromate gelatinoso juncta, ex epidermide erumpentia, demum effusa. (D enot. Micromyc. Ital. Dec. 11. pag. 80).

3:15. H. Grepini Desmaz, $0_{p}$ siervende en doode bladen van Camellia Japonica, in de oranjeriën. Hieme Vere.

Amphigena, atra, sparsa, approximata; sporidiis fusiformibus pedicellatis, utrinque hyalinis, $3-4$ septatis; articulo supremo appendicibus filiformibus coronato; filis 3, 4 tenuissimis, simplicibus, hyalinis, elongatis, divergentibus. (D es maz. Not. sur crypt. inéd. de la Fr. in Mém. de la Soc. Roy. des Sc. de Lille; Desnı a. Ann. des Sc. nat. Tom. XIII, année 1840 p. 183). In Herb.: Amsterdam, v. d. S. L a c. (v. v. et. s)

3214. rosae West. $O_{p}$ doode Rozentakjes. Ilieme. Périthèces épicaules, très-petits, bruns, épars et immergés, ostiole soulevant et déchirant irrégulièrement l'épiderme. Sporidies fusiformes à 3 cloisons transversales, formant $4 \operatorname{loges}$, dont la supérieure, surmontée de 2 cu 3 cils divergents, est hyaline et les 3 autres pâle brunâtres. La sporidie mesure de $1 / 50^{e}$ à $1 / 40^{\circ}$ de mill, de longueur sur $1 / 100^{\circ}$ de mill, de laryeur; les cils égalent la sporidie en longueur. (West. 6 e Not. cr. inéd. fl. B. in Bull. de l'Acad. Roy. des sc. de Brux. 2 Sér. T. VII. no. 70)

In Ilerb.: Amsterdam; v. d. S. Lac. (v. vo et s.)

THELOUHA $\mathrm{Fr}$.

Derithecium corneum, superficiale innatum vel imsersum, vertice poro perforatum vel irregulariter 
apertum, vel ostiolatum; ostiolo putictiformi, plus minusve cylindrico. Asci nulli; basidia filiformia vel stipitiformia, plus minusve elongata aut brevissima. Sporidiis terminalibus, ovoideis vel eliipsoideis, dein liberis; sporulis binis coloratis vel hyalinis, compressu. truncatis, hemi-ellipsoideis. (Fr. Emend. Desmaz. 17e Not. Gr. inéd, de la Fr. in Ann. des Sc, nat. $2^{e}$ Série, T. XI. Mai et Juin 1849. nº 14)

3215. H. inquinans West. $O_{p}$ doode Elzentronken en takken. Vere. (Sphaeria inquinans Tode; Fr. Syst. II. p. 486 ; R a be $\mathrm{nh}$. pag. 181)

In Herb.: Leiden, $\mathrm{W} t \mathrm{tw}$. ( $\mathrm{v}$. vo et $\mathrm{s}_{\text {.) }}$

3216. Wisci $\mathrm{Kx}$. Op doode takken, bladstelen en bladen van Viscum album. Vere. (K x. Rech. Cr. des Fl. 1e Cent. p. 21 ; Sphaeria atro-virens $a$ visci $\mathrm{A} / \mathrm{b}$. et Schw.; Fr. Syst. II. p. 501 excl. var. $\beta$.; Sph. visci Dec.; Rabenh. I. p. 171)

In Herb.: Bommel, W $t \mathrm{tw}$, ex herb, Jungh: (v. v, et s.)

321 \%. van Crataegus oxyacantha. Aut. Vere.

Périthèces noirs, globuleux, immergés, formant souvent des lignes paralelles et longitudinales, restant recouverts par l'épiderme déchiré en longueur. Ostiole papilliforme. Sporidies ovales, brunes, à une cloison. (West. $5^{\circ}$ Not. in Bull. de l'Acad. Roy. des Sc. de Br. 2e Sér. T. II. pag. 17; W est. Ilerb. Cr. Belge, $\left.\mathrm{n}^{0} . \mathrm{J128}\right)$

In Herb.: Amsterdam, v. d. S. Lac. (vi v. et s.)

3215. ID. cornil West. $O p$ doode takken van Cornus sanguinea. Aut. Vere.

Périthèces épars, noirs, immergés, restant reconverts par l'épiderne déculuré, qui dusiue sculement 
passage à l'ostiole papilliforme. Sporidies brunes, ovam les, biloculaires. (W est. loc. cit. p. 16)

In Ilerb. : Zuid-Beveland, v. d. B. (v. v. et s.)

3219. D. pinea $W$ est. $O p$ doode schors van Pinus sylvestris. Hieme.

Atra, erumpens; peritheciis ovoideis in seriem sinuosam dispositis, raro gregariis; ostiolo conico demum deciduo; sporidiis magnis, ovato ellipticis, brunneis, semiopacis, quandoque didymis aut uniseptatis. (Sphaeria pinea Desmaz. 9e Not. Cr. inéd. de Fr. in Ann. des Sc. nat. Févr. 1842. p. 14; Desmaz. Pl. crypt. de Fi. $n^{0} \cdot 1277$ )

In Herb.: Warmond, van Hall. (v. s.)

32R0. D. acerina $L_{e} v_{0} .0 p$ doode takken van Acer platanoides en Platanus orientalis. Vere.

Conceptaculis globosis innatis nigris in seriebus linearibus ordinatis epidermide fissa tectis, ostiolis obsoletis. (Lev. Champ, du Mius, de Paris in Ann. des Sc. nat. 2e Sér. T. V. p. 290)

In Ilerb.: Zuid-Beveland, v. d. B. (v. v. et s.)

32R1. T. rosarum Fr. Op stervende takken van Rosa cinnamomea. Hieme. Vere.

Périthèces épicaules, épars, noirs, sphériques, à ostioles papilliformes, déchirant l'épiderme noirci par transparence, pour le passage de la matière sporidifère. Sporidies ovales, biloculaires, étranglées à l'endroit de la cloison, et mesurant $1 / 40^{\circ}$ de mill. de longueur sur $1 / 100^{\circ}$ de mill. de largeur. (West. $6^{\circ}$ Nor. Cr. inéd. de la Fl. Belge in Bull. de l'Acad. Roy. des Sc. de Belg. 2 e Sér. T. VII. p. 21. no 75; West. H. cr. Belge $n^{\circ}$. 1227; Sphaeria spurca Wallr.? R abenh. p. 199)

In Herb.: Zuid-Beveland (Goes), จ.d. B. (v. vo et s.)

3a2. . . Verc. 
Pustules grosses, irrégulières, conlluentes, alteiruant jusqu'à 4 à 5 centim. de longueur sur une largeur de 4 à 5 millim., noires, saillantes et cntourées par les débris rélevés de l'épiderme. Périthèces globuleux, réunis par groupes dans les pustules. Sporidies ovales, brunes, biloculaires, mesurant $7 / 200^{\mathrm{e}}$ de mill. de longyueur sur $3 / 200^{\circ}$ de mill. de largeur. (West. 7 e Not. in Bull. de l'Acad. Roy. des Sc. de Brux. 2e Sér. T. XI. p. 6. $\mathrm{n}^{0}$. 2; West. H. cr. Belge $\mathrm{n}^{\circ}$. 1372; Sphaeria scabrosae affinis Rabenh. Herb. viv. myc. Ed. nov. $n^{\circ} .537$ ?)

In Herb.: Harderwijk, Jungh. (v. v. et s.)

3223. D. ligustri West. $O p$ doode takjes van Ligustrum vulgare. Hieme. Vere.

Périthèces très petits, globuleux, noirs, placés souvent par séries linéaires, recouverts par l'épiderme qui se déchire irrégulièrement pour montrer a nu un ostiole noir et papilliforme. Sporidies ovales, brunes, biloculaires. (W e st. $8^{\circ}$ Not. cr. inéd. A. B. pag. 7.)

In Herb.: Amsterdam, v. d. S. L a c. (v. v. et s.)

3224. Morl West. Op doode takken van Morus alba. Aut. Vere.

Périthèces très-petits, isolés, nombreux, noirs, recouverts par l'épiderme, qui se déchire irrégulièrement pour mettre a nu un ostiole papilliforme. Sporidies ovales, brunes, biloculaires. (W e s t. loc. cit. pag. 6.)

In Herb.: Austerdam, v. d. S. Lac. (v. v. et s.)

3225. D. conigena Desmaz. $O p$ de schubben van afgevallen kegels van Pinus maritima. Hieme. Vere.

Peritheciis immersis dein erumpentibus, subrotundatis gregariis subconfluentibus, atris, astomis. Nucleo nigro; sporidiis oblongis, utrinque obtusis, fuscis, unilocularibus. (Desmaz, 13e Not. in Ann. des Se. nat. p. 09. 11\% 12; Hhoma pinastri Lev.; Hacron 
plodia conigena West. Herb, cr. Belge $\mathrm{n}^{\circ}$. 1230 \}

In Herb.: Ilarderwijk, Jungh. (vo vo et s.) $^{\text {) }}$

3226. ID. Salicis West. $O_{p}$ doode takken van Salix alba en andere. Aut, Vere.

Périthèces membraneux, sphériques, d'environ $2 / 3$ de mill. de diamètre, s'affaissant par la sécheresse, noirs, épars, recouverts par l'épiderme noirci par transparence. Ostiole papilliforme. Sporidies hyalines, fusiformes, de $3 / 200^{\mathrm{e}}$ de mill. de longueur sur $1 / 300^{\mathrm{e}}$ de mill, de largeur, à une cloison au milieu. (Diplodina salicis West. 5e Not. pag. 19. no. 28, fig. 6 ; W es t. Herb. crypt. B. $n^{\circ}$. 1229)

In Herb.: Utrecht, v. d. S. Lac; Rijnsburger weg,

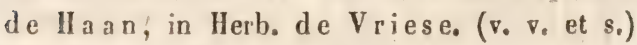

\section{DISCDSIA Lib.}

Perithecium innatum scutiforme ostiolo perforatum obtegens ascidia fusiformia utrinque in productionem filiformem protensa, sporidiis globosis. (Lib. Pl. crypt. Ard. $\left.n^{0} \cdot 345\right)$

3228, ID. artocreas Lib. Op doode Beukenbladen. Hieme. Vere. (Lib. 1. 1. $n^{\circ}$. 345; Sphaeria artocreas Tode; Fr. Syst. II. p. 523; Rabenh. p. 170)

In Herbs: Utrecht, vo d. S. Lac。 - Bovendien: Maastricht, Franq. (v. vo et s.)

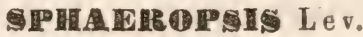

Perithecium sphaericum, immersum, subinnatum, astomum, demum (epidermide secedente) circumscis. sum 1. irregulariter rumpens. Sporae simplices absque ascis. (Lev. in Demid. Voy.)

3zage, 5ph. aepressa Lev. Op doode takjes ran $S_{y-}$ ringa vulgaris. Hieme. Vere. 
Conceptaculis gregariis innatis hemisphaericis niwris demum collapsis epidermide rupta tectis, ostiolis papillatis pertusis, sporis cylindrico-ovatis obtusis. (Lev. Champ. du llus, de Par, in Ann. des Sc, nat. $2^{e}$ Sér. T. V. p. 295)

In Herb.: Harderwịk , Jungh. (v. s.)

3229. ฐph. fimicola West. $O p$ verdroogde kocmest. Aut. Vere.

Périthèces très-petits, ponctiformes, sphériques, noirs, luisants, d'abord immergés, puis devenant superficiels. Ostiole papilliforme. Sporidies grandes, hyalines, ovales, uniloculaires, de 2 à $3 / 100^{e}$ de mill. de longueur sur $1 / 75^{\mathrm{e}}$ de mill, de largeur. (West. $5^{\mathrm{e}}$ Not. crypt. inéd. de Belg. pag. 20. $\mathrm{n}^{\circ}$. 31. fig., 9)

In Herb.: Zuid-Beveland (Goes), vo d. B. (v. v. et s.)

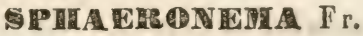

32319. Sple. cylindricum F r. $O_{p}$ rottend hout, hijzonder van Wilgen en Eiken. Aut. Vere. (F r. Syst. II. p. 538 ; Rabenh. p. 149)

Maastricht, Franq. (n. v.)

3231. Sph. conicum Fr. Op' doode stengels van groote kruidgewassen. Aut; Vere. (Fr. Syst. II. p. 538; R aben h. p. $149 ; \mathrm{Dz}$. et II b. Bijdr. I. p. 22)

Leiden, Dz. et $\mathrm{M} \mathrm{b}$. (n, v.)

3232. Sph. truncatum $\mathrm{Fr}$ : $\mathrm{Op}$ rotlend hout van Hasten Pịnboomen. Aut. Vere. (F r. Syst. II. p. 539; R abenh. p. 149 ; Dz. et MI b. Bijdr. I. p. 22)

In Herb.: Leiden, $\mathbf{D}$ z. et $\mathrm{H}$ b. (v. s.)

3233. Sph. anemones $L_{i}$ b. Op de bladen, stelen en bloemen van Anemone nemorosa. Vere. (Lib. Pl. crypt. Ard. no. 167; Sphacria anemones Fr. Syst. II, p. 563 ; Rabenh. p. 189)

Maastricht. Franq. (n, v.) 


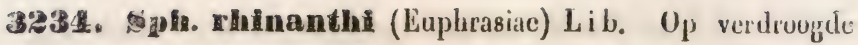
zaadhuisjes van Euphrasia odontites. Aut. Hieme.

Peritheciis sparsis, rotundis plano depressis, poro simplici pertusis, nigris, globulo sporophoro minutu albo. (L i b. Pl. crypt. Ard. nº. 263)

Ii llerb.: Zuid-Beveland, v. d. B. (v. v. et s.)

\section{SPORONERI Desmaz.}

Perithecium innatum, epidermide tectum dein crum。 pens, sessile, subimmersum ab initio clausum demuus dehiscens a centro versus ambitum in lacinias plures. Nucleus discoideus tremelloso-gelatinosus, basidiophorus; basidia filiformia, simplicia vel dichotoma, 1-poJyspora. Sporae hyalinae, rectae, ovoideae vel cylindricac. (Des maz. 19e Not. crypt. inéd. de Fr. p. 24)

3235. Sp. ramealis Desmaz, $O_{p}$ doode takken vau Sambucus nigra. Hieme et Vere.

Numerosa, dense sparsa, minutissima, rotundata vel oblonga in lacinias (3.4) inaequales dehiscens. Disco albido rel rufo, convexo. Sporidiis hyalinis, rectis, oblongo-cylindricis, obtusis; sporulis 2-3, globosis quandoque subopacis. (Des in az. 1. 1. p. 25)

In Herb.: Zuid-Beveland (Goes), vo d. B. (v. s.)

3236. Sp. Strobilina Desmaz. $O_{p}$ de schubben vau rottende kegels van Pinus abies. Hieme.

Peritheciis minutis subglobosis, tenuissimis, atris, gregariis quandoque confluentibus in lacivias plures inaequales irregulariter dehiscentibus. Disco convexo, gelalinoso, griseo, subopalino. Sporophoris simplicibus, monosporis; sporis numerosis, hyalinis, fusiformibus. (Desmaz. 20 Not. Crypt. inéd. de Fr. po 14. $\left.\mathrm{n}^{\circ} \cdot 16\right)$

In Herb.: IIarderwijk, W $l$ w. ex herb. Jungh. (v, so) 


\section{PHLHEIU⿴囗十⺝ $\mathrm{Kze}$.}

Perilhecium innatum, hemisphaericum, rimis plurio bus a centro ruptum, sporidiis fusiformibus refertum. (K z c. Hyc. Hefte H. p. 92)

323\%. IP. carbonaceum $\mathrm{Kze}$. Op doode Wilgentakken. Vere. (K z e. Mlyc. IIefte II. p. 92. Phacidium carhonaceum Fr. Syst. II. p. 574; Rabenh. p. 160)

In Herb.: Leiden, $\mathrm{W}_{\mathrm{t} t} \mathrm{w}$. (v. s.)

\section{CEU THIPIIEA $\mathrm{Fr}$.}

3235. C. phacidioldes Grev. Op stervende en doode bladen van Ilex aquifolium. Aut. (F r. Syst. II. p. 576; Rabenh. p. 144)

In Herb.: Leiden in den Hortus academicus, W 1 w.

3239. C. umbonata $\mathrm{W}$ allr. $0 p$ de bladen van Cochlearia anglica. Aut. ( $\mathrm{R}$ a be $\mathrm{n}$. p. 144; W allr. Comp. Fl. Germ. IV. p. 746)

In Herb.: Aan het IJ te Amsterdam, v. d. S. La c.

\section{CHIEILATIR L i b.}

Perithecia difformia, rotundata, rima dehiscentia; asci gelatina juncti subcirrhose rejecti; sporidia globosa. (L ib. Pl. crypt. Ard. Cent. 1. pag. 8)

32110. Ch. agrostidis Lib. Op de bladen van Agrostis vulgaris en andere grassoorten. Aut.

Maculis fusco-albescentibus aut nullis. Peridiis immersis, utrinque prominulis, ovatis elongatisve atris, rima longitudinali dehiscentibus; cirrhis albis; ascis fusiformibus; sporidiis 2 pellucidis. (Lib. Pl. crypt. Ard. $\mathrm{n}^{\circ}$ 63)

In Ifcrb.: Zuid-Berolaud, vo d, B. (v. so) 


\section{MHLATMA $L \mathrm{ev}$.}

Conceptacula membranacea apice dehiscentia tumio dula demum depressa rugosa receptaculo tenui effuso maculaeformi innata. Sporae minutae sublineares continuae obtusae pellucidae in globulum gelatinosum erumpentes. (Lev. Champ. du Mlus, de Par. in Ann. des Sc. nat. 2e Série T. V. p. 276)

321. I. F. acerina L ev. Op levende en treurende bladen van Acer pseudoplatanus. Aut.

Receptaculis epiphyllis sparsis vel confluentibus maculas nigras suborbiculares pustulosas zona discolore cinc. tas efficientibus. (L e v. I. I.)

In Herb.: Haamstede, vo d. B. (v. vo et s.)

Obs. Ne pas confondre avec le Rhytisma punctatum, dont il a plusieurs traits de ressemblance.

\section{THEMA $\mathrm{Fr}$.}

3219. Ph. pustula Fr. Op doode Likenbladen. Vere. (Fr. Syst. II. p. 547; Rabeih. p. 143; Sphaeria pustula Pers.)

In Herb.: Leiden, Stek h. (v. s.)

3243. Fh. Hongissima W est. Op doode stengels van verschillende soorten van Chenopodium. Hieme. Vere.

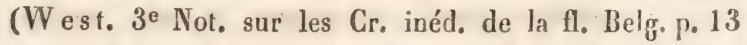
$n^{\circ} 45$; Sphaeria longissima Pers.; Fr. Syst. 11. p. 430; Rabenh. p. 191)

In Herb.: Utrecht, v. d. S. Lac.; Zuid-Beveland, v. d. B. (v. v. et s.)

3214. Ph. albicans $\mathrm{D}$ esmaz, $0 p$ de bloemstelen van Hypochoeris glabra. Aut.

Peritheciis minutis, numerosis, inordinatis, intus sordidis, epidermide tectis, humidis gुlobosis prominentibus; ostiolis nudis, minutis, papillatis; sporidie oblon- 
gis, sublinearibus. (D esmaz. 170 Not. Crypts inéd. Fr。 pag. 12. $n^{0} 20$; Desmaz. Pl. Cr. de Fr. $n^{\circ} 1865$ )

In Ulerb.: Berg en daal bị Nijmegen, A bel. (v. v. et s.)

3245. Ph. atriplicinum W est. $O_{p}$ doode stengels ran Atriplex hortensis. Aut. Hieme.

Périthèces membraneux, ovale-allongés, noirs, réconverts par l'épiderme noirci. Ostiole papilliforme. Sporidies ovales, hyalines, de $1 / 100^{\mathrm{e}}$ de mill. de longueur sur $1 / 200^{\mathrm{e}}$ mill. de largeur et contenant deux sporules globuleuses aux extrémilés. (W est. 5e Not. Crypt. inéd. de la Belg. p. 20. no 35; West. Herb. Cr. B. no 1233)

In Herb.: Mlaastricht, Franq. (v. v. et s.)

3216. IPh. Complamata Desmaz. Op doode stengels van Umbelliferae en bijzonder van Heracleum en Angelica. Aut. Vere. (D esmaz. 19e Not. Cr. nouv. de Fr. p. 4; Sphaeria herbarum var. complanatum Pers.; var。 major Rabenh. p. 175; Sph. complanata Tode; Fr. Syst. II. p. 508; Dz. et IIb. Bijdr. I. p. 21)

In Herb.: Rijnsburg, de II a an; Leiden, Utrecht, $\mathrm{W} t \mathrm{tw}$; B Bauwkapel, v. d. S. Lac. (v. v. et s.)

324.8. Ph. coromillae West. Op doode takjes van $C_{0}$ ronilla emerus en varia. Aut. Hieme. (West. Herb. Cr. B. $\mathrm{n}^{\circ}$ 966; Spaeria coronillac Desmaz. PI. Cr. de Fr. $n^{\circ} 1279$; Desmaz. Not. in Ann. des Sc. nat. 2 Sér. Tom. XIII. p. 188)

Sparsa, approximata, subgregaria; peritheciis immersis, tectis, minutissimis, subgylobosis, albido farctis; ostiolo simplici pertusis. Sporidiis liberis, oblongis, 1/100 millemetro longis; sporulis 2, globosis. (Desmaz. I. l.)

In llerb.: Zutphen, W $t$ tw. (v. v. et s.)

3215. Ph. equiseti Lev. (") doode stengels van Equisetum palustre. Aut. 
Conceptaculis innatis sparsis, glob sis minutis intus nigris epidermide obtectis, ostiolis obsoletis, sporis ovatis pellucidis simplicibus. (Lev. Champ. du Mus. de Par. in Ann. des Sc. nat. 2e Sér. Tom. V. p. 282; West, Herb. Cr. B. $n^{\circ}$ 632)

In Ilerb.: Zuid-Beveland, v. d. B. (v. v. et s.)

3249. Ph. herbarum West. Op doode stengels van Urtica dioica en Cirsium lanceolatum. Vere.

Périthèces membraneux, translucides, très-petits, ovales ou arrondis, bruns, d'abord recouverts par l'épiderme, qui se détruit et laisse le perithèce à nu. Ostiole poriforme bien apparent. Nucleus blane, sortant par l'humidité sous forme de cirrhe et se resolvant en un très grand nombre de sporidies ovalaires, transparentes, très-petites, contenant 1 ou 2 sporules aux extrémités. (West. 3e Not. Crypt. nouv. Fl. B. pag̣. 15 $n^{\circ}$ 51; West. Herb. Cr. B. $n^{\circ}$ 965)

In Herb.: Leiden, W $t \mathrm{t}$ w.; Utrecht, vod. S. La c.; Amsterdam, v. d. S. La c.; Katwijk, Zuid-Beveland, v. d. B. (v. v. et s.)

3250. IPh. Striaeformis Dur. et Il ont. Op doode takken van Kerria Japonica. Aut. Vere. (Hont. Syll. gen. spec. crypt. p. 270; Desmaz. Pl. Crypt. de Fr. nouv. Ed. $\mathrm{n}^{\circ} 59$; Sphaeria striaeformis Fr.? Syst. 11. p. 428; Rabenh. p. 191)

In Herb.: Amsterdam, v. d. S. L a c. (v. v. et s.)

3251. Ph. lineolatum Desmaz. Op de schubben van rottende kegels van Pinus larix en pinaster. Hieme et Vere.

Subtectum, erumpens, nigrrum. Peritheciis membranaceis tenuissimis, seriatis, subconnatis, globosis, stromate albido inmersis, intus albis, poro pertusis. Cirthis tenerrimis tortis, albis. Sporidiis hyalinis, ovoideooblongis. (Desmaz, 19e Nol. Cr. inedd, de la fl. Fr. 
p. 3; Desmaz. Pl. Crypt. de Fr. nonv. Fd. no 5s)

In Ilerb.: Deventer, W $t \mathrm{t}$ w.

3252. Ph. podagrariae West. Op de levende en treurende bladen van Aegopodium podagraria. Aut. (Dothidea podagrariae Fr. Syst. II. p. 556; Dz. ct $\mathrm{Ml}$ b. Bijdr. I. p. 22; Sphaeria podagrariae R ot h.; R a be n h. p. 188; Phoma West. Herb. Cr. B. $n^{\circ}$ 722)

In Herb.: Sassenheim, Oudem.; Utrecht, v. d. S. Lac. (v. v. et s.)

3253. Ph. ruborum West. $0 p$ doode ranken van verschillende Rubus-soorten. Hieme. Vere.

Périthèces épars, immergés, arrondies ou ovales, assez grands, atteignant jusqu'à 2 mill. dans leu gorand diamêtre, saillants, bruns et luisants. Ostiole nul. Sporidies hyalines, cylindriques, a extrémités arrondies, droites ou légèrement courbées, mesurant $3 / 400$ e de mill. de longueur sur $1 / 400$ e de mill. de largeur. (W e s t. 6e Not. Cr. inéd. fl. Belg. p. 22. no 79; West. Herb. Cr. Belg. ${ }^{\circ}$ 1234)

In Herb.: Zuid-Beveland, v. d. B. (v. v. et s.)

3254. Ph, samarorum Desmaz. Op de zaadhuisjes van Esschen. Hieme. Vere.

Tubercules noirs, orbiculaires et convexes, s'ouvrant chacun par un orifice arrondi, ensuite un peu allongé et à bords blanchâtres. Les sporules sont oblongues et n'ont pas plus de ${ }^{1 / 150^{e}}$ de mill. de longuear. (Desmaz. Observ. crypt. in Mlém. de la Soc. Roy. des Se. de Lille, année 1827-28. p. 452; Desmaz. PI. Cr. do Fr. no 349 ; Dz. et Ml b. Bijdr. II, p. 17)

In Herb.: Zuid-Beveland (Goes), v. d. B. (v. s.)

3255. Ph. siliquastrum Desmaz. $0 p$ de zaadhuisjes van Brassica. Aut. Hieme.

Maculis oblongis, olivaceo-brunneis; peritheciis numerosis, mainutissimis, approximatissimis, fusco-mizris, 
poro apertis; sporidiis peresilibus, oblongis; sporulis 2 globosis, opacis. (Desmaz. 17e Not. Cr., nouv. de Fr. p. 8 n $^{\circ} 12$; Desmaz. Pl. Cr. de Fr. n० 1874)

In Herb.: Zuid-Beveland (Goes), v. d. B. (v. vo)

\section{PPIILLETTETA Pers. Ch. Comest.}

Perithecia exilissima, innata, poro aperta, maculas decoloratas in foliis procreantia. Nucleus gelatinosus; ascis nullis; sporidiis ovoideis, vel oblongis rectis, minutissimis, subcirrhose rejectis. (Desmaz. 14e Not. crypt. nouv, de Fr. p. 28)

3256. Hh. atriplicis $W$ est. $O p$ treurende bladen van Atriplex angustifolia en patula. Aut. (Sphaeria (Depazea) vagaans $d$ atriplicicola Fr. Syst. II. p. 532; Rabenh. p. 139; Dz. et $\mathrm{Ml}$ b. Bijdr. I. p. 21)

In Herb.: Leiden, Dz. et II b. - Bovendien : Mlaastricht, Franq. (v. v. et s.)

325\%. DP. berberdis West. $O p$ levende bladen van Berberis vulgaris. Aut.

Taches très petites ( 1 ̀̀ 3 mill.), anğ ches, entourées d'une ligne rougeâtre, éparses ou confluentes. Périthèces noirs, ponctiformes, au nombre de 2 à 3 sur chaque tâche. Ostiole poriforme; sporidies ovales, hyalines, de $1 / 100^{\mathrm{e}}$ de mill. de longueur sur $1 / 200^{e}$ de mill. de largeur, contenant deux sporules globuleuses. (West. 5e Not. Crypt. inéd. de la fl. Belgo p. 24. $n^{\circ}$. 54; W est. Herb. Crypt. Belge ${ }^{0}$. 1375)

In Herb.: Kampen, Bond. (v. v. et s.)

æฆ5ร. Th. Trassicae We s t. Op stervende Koolbladen. Aut. (W est. 2e Not. crypt. inéd. de la fl. Belg. p. 20, no 92; Septoria brassicae W est. Herb. Crypt. Belg. $\mathrm{n}^{0}$. 294; Depazea brassicaecola Duby; Kabenh. p. 138)

Maastricht, Franq. (n. v.) 
325פ. Fh. camellace West. $O p$ treurencie bladen vat Camellia japonica in de oranjeriën. Ilieme. (W est. 20 Not. Crypt. inéd. de la Belg. p. 21. nº 96)

Taches arrondies, assez grandes ( 2 à 3 centim.), se développant indifféremment sur les bords ou le centre de la feuille, brunâtres, devenant blanchâtres avec l'âge, entourées d'une bordure étroite, épaissie, brun pourprée. Perithèces immergés, noirs, noircissant quelquefois l'épiderme par transparence, épars sur toute la tache. Cirrhe blanchâtre. Sporidies ovales, très petites transparentes, contenant aux extrémités 2 sporules globuleuses. (W e st. 1. 1.)

In IIerb.: Amsterdam, v. d. S. La c. (v. v. et s.)

3260. Illa. coryli $W$ est. $O p$ de treurende bladen van Corylus Avellana. Aut. (W est. 3e Not. Cr. inéd. fl. B. pag. 18 no. 76 ; West. Herb. Cr. B. no. 731.) Taches hypophylles, arrondies, brunes, assez grandes, de 5 à 15 mill. de diamètre. Perithèces épars sur toute la tache, brun-noirâtres, petits, percés d'un pore simple, Cirrhe blanchâtre. Sporidies ovales, contenant 2 sporules hyalines placés aux extrémités.

In Ilerb.: Utrecht, v. d. S. Lac. (v. v. et s.)

3261. Ph. cruemta Kx. Op treurende bladen van Convallaria multiflora en polygonatum. Aut. (K x. Rech. Crypt. des Fland. 4e Cent. p. 22; Sphaeria (Depazea) cruenta Fr. Syst. II. p. 531; D z. et II b. Bijdr. I. p. 21; Depazea cruenta Kze; Rabenh. p. 138)

In IIerb.: 's llage, D z. et $\mathbb{I I}$ b.; Wassenaar, J u ng h.; Leiden, $\mathbf{D} z$. et $M b$. ( $\boldsymbol{v}_{\text {. }}$ v. et $s$ )

3262. Ph. destuctiva $\mathrm{D}$ esmaz. Op stervende bladen van verschillende Malva-soorten. Aut. (Ph. destructiva B. malvacearum Desmaz. 14e Not. Crypt. de Fr. p. 29 ; Sphaeria (Depazea) vagans d. malvaecola $\mathrm{Fr}$. Syst. II. p. 532; Rabenh. p. 139). 
In Herb.: .... Lantz. Ben. - Bóvendien: Naastricht $\mathrm{F}$ ran $\mathrm{q}$.

3263. Hh. fragariaecola $\mathrm{R} o \mathrm{~b}, 0 \mathrm{p}$ stervende en treurende bladen van Fragaria vesca. Aut. (Rob. in Desmaz. Pl. Crypt. de Fr. nouv. Ed. nº 686; Depazea fragariaccola Wallr.; Rabenh. p. 138; Spbaeria ragans $\gamma$. fragariaecola $\mathrm{D} z$. et $M \mathrm{~b}$. Bijdr. I. p. 21)

In Herb.: Leiden, $\mathbf{D}$ z. et $\mathbf{M}$ b. (v. s.) - Bovendien: Maastricht, F ranq.

3264. Ph. merearialis Desmaz. Op treurende bladen van Mercurialis annua. Aut.

Naculis albis, exaridis, minutis, indeterminatis, interdam confluentibus. Peritheciis amphigenis, innatis, pallide succineis, dein fusco-nigricantibus, poro apertis. Sporidiis hyalinis, rectis, oblongis, atrinque obtusis, 1-septatis, vel 3,4 guttulis repletis. Desmaz. 17 e Not. Cr. de Fr. p. 25 nº 35 ; Desmaz. Pl. Cr. de Fr. $n^{\circ} .1857$ )

In Herb.: Hees bij Nymegen, A bel (v. v. et s.)

3265. Hh. Thodondendri West. $O p$ treurende bladen van Rhododendron arboreuin in de oranjeriën. Hieme,

Taches rousses ou brun rougeâtres, assez grandes (3 a 5 centim.), indéterminées, attaquant ordinairement les bords ou le sommet de la feuille. Périthèces immergés, saillants, noirs à ostiole poriforme assez grand. Cirrhe incarnat. Sporidies cylindrique-ovales, contenant 3 ou 4 sporules, globuleuses. (West. $2^{e}$ Not. Crypt. inéd. de la Belgo. pag. 22 n. 100)

In Herb.: Leiden, Dz. et II b., 0 udem. (v. s.)

3266. Ph. rumicis Pers. $O_{p}$ treurende bladen van $R u$ mex acetosa etc. Aut. (Pers. Ch. comest: p. 147. Sphaeria vagans v. rumicicola Fr. Syst. II. p. 532; D. et $M b . B i j d r, I, p, 21$ ) 
In Ilerb.: Leiden, $\mathbf{D}$ z。 et $\mathbf{M} b$; Zuid-Beveland, v. d. B. (v. s.)

3268. Ph. sambucl Desmaz. Op treurende bladen van Sambucus nigra. Aut.

Epiphylla. Maculis candidis, solitariis vel confluentibus in series dispositis. Peritheciis curvato-prominulis, minutissimis, paucis fusco-nigris, poro apertis. Nucleus albidus; sporidiis perexiguis, ovoideo-oblongis, bimaculatis. (Desmaz. 14e Not. Grypt, de Fr. pago 34. $\left.\mathrm{n}^{\circ} .51\right)$

In Herb.: Broek in Waterland, Kra a m.; Kampen, R. Bond. (v. vo. et s.)

3265. Ph. ulmi West. $O_{p}$ treurende en stervende bladen van Ulmus campestris. Aest. Aut.

Taches amphigènes, arrondies, éparses ou confluentes, d'un blane-grisâtre sale, sans bordure ni aréole et souvent stériles. Perithèces rares, excessivement petits, éparpillés sans ordre sur toute la tache. Ostiole poriforme. Sporidies ovales, hyalines, de $1 / 100^{\mathrm{e}}$ de mill. de longueur sur $1 / 200^{\circ}$ de mill. de largeur. (West. 5. Not. Crypt, inéd. de la Belg. p. 27. no 69 ; W est. llerb. Crypt. B. $\mathrm{n}^{\circ}$. 1143)

In Herb.: Amsterdam, v. d. S. L a c. (v, 8.)

3269. Ph. Wiolae Desmaz. Op treurende bladen van Viola odorata. Aut.

Amphigena. Maculis albidis, rotundatis, sparsis rel confluentibus. Peritheciis innatis, numerosis, microscopicis, fuscis. Cirrhis albis. Sporidiis minutissimis, rectis, subeylindricis. (Desmaz. 140 Not. Crypt. de Fr. p. 29. $\mathrm{n}^{\circ}$. 41)

In llerb.: In den kruidtuin van Nymegen. $\mathrm{A}$ be $\mathrm{I}$. 
CVTIS POIE (Ehrenb.) Fr:

3280. C. Chrysosperma Fr. Op doode takken van Po: pulus tremula. Per annum. (F r. Syst. II. p. 542; Rabenh. p. 146)

Maastricht, Fran q. (n. v.)

3281. C, corni West. $0 p$ doode takken van Cornus sanguinea. Aut.-Vere.

Périthèces noirs, membraneax, comprimés, très petils, réunis par groupes de 3 ou 4 , recouverts par l'épiderme soulevé et bruni; les ostioles de chaque groupe sc réunissent pour percer l'épiderme au centre de la partie tachée, sous forme de disque ponctiforme, noir et luisant. Cirrhe d'un blanc jaunâtre, formé par l'aggromération de sporidies excessivement petites, cylindriques, droites ou légèrement courbées, de $1 / 200^{\mathrm{e}}$ de mill. de longueur sur $1 / 800^{\circ}$ de mill. de largeur. (W es t. 5e Not. Crypt. inéd. de la Belg. pag. 28. no 76; West. Herb. Cr. Belg. $n^{\circ}$. 1129)

In Herb. : Utrecht, จ. d. S. L ac.; Zuid-Beveland, v. d. B. (v. v. et s.)

3282. C. fugax $\mathrm{Fr}$. Op doode Wilgentakken. Per annum. (Fr. Syst. II. p. 544; Rabenh. p. 146)

In Herb.: Warmond, Rhijnsburg, de II a a $\mathbf{n}$, in Herb. de Vriese; Leiden, Ankersm.; Utrecht, W ttw.; Amsterdam, v. d. S. Lac.; Goes, v. d. B.; Maastricht, Franq. (v. v. et s.)

3283. C. Incarnata $\mathrm{Fr}$. $\mathrm{Op}$ doode takken ran Robinia inermis en pseudo-acacia. (Fr. Syst. II. p. 542; Rabenh. p. 148),

In Herb.: Leiden, $D_{z}$, et $M$ b. (vo vo et $s_{\text {s }}$ )

3284. C. Icucosperma Fr. Op doode Populiertakken. Per annum. (Fr. Syst. II. p. 543; R abenh. p, 147; Dz, et..ll b., Bijdr. t. I. p. 22) 
In Herb.: Utrecht, v. d. S. Lac.; Amsterdam, 0 udem.; v. d. S. Lac. (v. vo et s.)

3285. C. macillenta Desmaz. $O p$ doode takken van Cornus sanguinea. Aut.-Vere.

Pustulis minutis, punctiformibus, brunneis ve nigris, numerosis, inordinatis, irregulariter rotundatis, ovatis vel reniformibus, convexis, epidermide tectis dein rupta applicatis; cellulis $5-7$ tenuibus, circinantibus; nucleo pulposo; cirrho crasso, abbreviato, albido-lutescente, Sporidiis oblongis, rectis, obtusiusculis, hyalinis; sporulis 2-4, globosis, subopacis. (Desmaz. 17e Not. Crypt. de Fr. p. 26. no. 37; Desmaz. Pl. Crypt. de Fr. no ${ }^{0}$ 1898)

In Herb.: Leiden, Dz. et $\mathbf{I l}$ b.; Utrecht, v, d. S. L a c. (v。 vo et s.)

\section{BHCEDIDER L E V .}

Conceptacula innata membranacea ovato-elongata vertice dehiscentia. Sporae lineares continuae curvatae cum gelatina inmixtae in massam erumpentes. (L é v. Champ. du mus. de Par. in Ann. des Sc, nat. $2^{\circ}$ Série Tom. V. p. 283)

3276. NI. Arupacearum Lév. $O_{p}$ doode schors van Krieken en Pruimen. Per annum. (Lév. I. l.)

Conceptaculis pustulatis innatis subeylindricis basi coadunatis farinaceis transversim erumpentibus. ostiolis albis, sporis apice curvatis. (Lév. 1.1.; Cenangium cerasi var, junior Fr. Syst. myc. II p. 180).

In Herb. : Amsterdam, v. d. S. L a c. (v. vo et s.)

Obs. Cette espèce, serait d'après Mr. Tulasne, l'état spermogonique du Cenangium Cerasi Fr.

SERTIIEA Fr. (Ascochgta Lib.)

38: 8. S. acgopodii Desmaz, Op treurende bladen van 
Aeggopodiam podagraria Aut. (Des maz. Pl. Crypt de Fr. $n^{\circ}$ 616; Sphaeria aegopodii Pers.; Dz. et Mlb. Bijdr. I p. 21 ; Fr. Syst. II. p. 526; R abenh. p. 580). In Herb.: Leiden, Dz. et Il b.; Utrecht, W t tw.; Rotterdam, 0 u dem.; Naaldwijk, v. d. Tr.; Zwake, v. d. B. - Bovendien: Maastricht, Franq. (v. v. et s.)

3285. S. aesculicolla West. Op stervende bladen van Aesculus hippocastanum. Aut. (W e s t. 2e Not. Crypt. inéd. Belg. p. 17. $\mathrm{n}^{\circ} 80$; Sphaeria (Depazea) aesculicola Fr. Syst. II. p. 530; Rabenh. p. 137; Dz. et 11 b. Bijdr. I. p. 21).

In Herb.: Leiden, $\mathbf{D} z$. et $\mathbf{K} \mathrm{b}$. (v. v. et s.)

3289. \$. allorum iVest. $O p$ de bladen van Allium porrum. Aest. Aut.

Taches irrégulières, éparses ou confluentes, non limitées, verdâtres, devenant d'un blanc de lait au centre, dépassant rarement un centim. de diamètre. Perithèces nombreux, sémi-immergés, éparpillés sans ordre sur toute la tache, d'un roux-brunâtre, à ostiole poriforme. Sporidics flexueuses; cylindriques, obtuses aux extrémités, contenant de 4 à 6 sporales globuleuses. (W est. 2 Not. Crypt. inéd. de la Belg. pag. $19 \mathrm{n}^{\circ}$ 86.)

In Herb.: Amsterdam, v. d. S. Lac. (v. s.)

3280. \$. arî Desmaz. Op treurende bladen san Arum maculatum. Aest. Aut.

Maculis amphigenis, parvis, albido-exaridis vel virio dulis suborbiculatis, fusco-cinctis. Peritheciis epiphyllis, paucis, minutis, brunneis, innato-prominulis, poro apertis dein collabescendo concavis. Sporidiis rectis, linearibus; sporulis globosis numerosis. (Desmaz. $14^{\mathrm{e}}$ Not. Crypt. de la Fr. pag. $\left.18, \mathrm{n}^{\circ} 19\right)$.

In Herb, : Utrecht, $\mathbf{D}$ z。 et $\mathrm{M} b$. (v. v. et s.)

3251. 5. Betae West. Op treurende bladen van Bete vulgaris. Aest. Aut. (Sphacria (Depazea) ragans $h$. 
befaccola Dec.; Fr. Syst. 11. p. 532; Rabenh. p? 139; West. 2e Not. Crypt. inéd. Belgु. p. 16, $\mathrm{n}^{\circ} 77$ ). Maastricht, Franq. (n. v.)

2:89. 5. cheiranthi Rob. $0 p$ treurende bladen van Cheiranthus cheiri. Aest.

Maculis amphigenis, helvolis, rotundatis vel irregularibus. Peritheciis epiphyllis, paucis, nigrris, innatoprominulis, poro pertusis, dein collabescendo concavis. Cirrhis eburneo-subcarneis. Sporidiis tenuissimis, curvulis, sporulis 6-8. (Desmaz. 14e Not.p. 20, n²5).

In Herb.: Deventer ? (

3253. \$. chelidonil Desmaz. Op treurende bladen van Chelidonium majus. Aest. Aut. (Desmaz. Pl. Crypt. de Fr. $n^{\circ}$ 1176; Sphaeria lichenoides var. Chelidonicola Dec.; Ascochyta Lib.; Raben h. p. 146).

Maastricht Franq. (n. v.)

3254. $\$$. chenopodil West. $0 p$ treurende bladen van Chenopodium viride en anderen. Aest. Aut.

Taches arrondies, éparses ou confluentes, atteignant rarement 6 millim. de diamètre, d'un blanc verdâtre, devenant avec l'âge d'un jaune sale, entourées de zones jaunes ou brunâtres. Perithèces innombrables, noirs, sémi-immergés, très petits, éparpillés sur toute la tache et lui donnant une teinte plombée: Ostiole poriforme. Cirrhe blanchâtre. Sporidies droites ou flexueuses, cylindriques ou fusiformes obtuses aux extrémités et contenant de 6 à 10 sporules globuleuses. (W est. $2 \mathrm{e}$ Not. Crypt. inéd. de la Belg. p. 19, n 88; West. Herb. Cr. B. ${ }^{\circ}$ 643).

In Herb.: Tjepma ... ? (v. v. et s.)

3285. S. convolvuli Desmaz. Op treurende bladen van Convolvulus arvensis en sepium. Aest. Aat. (Desmaz. Pl. Crypt. de Fr. no 1172; Sphaeria (Depazea) Gentianaccola of Convelsulicula Dec, Fr. Syst: 
11. p. 531 ; Depazea Concolvulicola Rabenh. p. 138).

In Herb.: Bij Nijmegen, A b el. - Borendien: Haastricht, Franq. (v. v. et s.)

3286. Cornicola Desmaz. Op bladen van Cornus sanguinea. Aest. Aut. (Des maz. Pl. Crypt. de Fr. $n^{\circ} 342$; Sphaeria (Depazea) cornicola Dec。; Fr. Syst。 II. p. 530; Depazea cornicola R a benh. p. 137).

In Herb.: Leiden, Dzo et $\mathbf{M I}$ b. - Borendien: Maastricht, Franq. (vo v. et $s_{0}$ )

3288. D. Uianthil Desmaz. Op treurende bladen vau Dianthus barbatus. Aest. Aut. (Desmaz. Pl. Crypt。

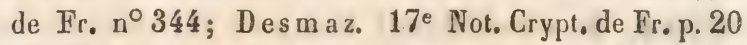
n० 27; Sphaeria (Depazea) Dianthi Fr. Syst. II. p. 531; Depazea dianthi Rabenh. p. 137; Dz, et II b. Bijar. I. p. 21)

In Herb.: Scheveningen, $\mathrm{D}_{\mathrm{z}}$ et $\mathrm{Mb}$; ; Bommel, Jungh. in Herb. W ttw. (v. v. et s.). Variat:

a. Saponariae Desmaz. I.1. Op bladen van Saponaria officinalis.

In Herb.: Katwijk, W $t$ tw, ex herb. Ankersm. (v. v. et s.) - Bovendien: Maastricht, Franq.

3285 \$. Lcariae Desmaz, $O_{p}$ treurende bladen van $R a$ nunculus ficaria. Aest. Aut.

Amphigena; maculis parvis, orbiculatis, fuscis, demum cinereis exaridis, in ambitu fuscis, irregularibus vel confluentibus; peritheciis innatis, minutissimis, nigris, subnitidis, convexis, demum planiusculis; cirrhis albis; sporidiis linearibus tenerrimis, rectis vel subcurvatis. (Desmaz. 8e Not. Crypt. nouv, de Fr. p. 7; Desmaz。 Pl. Crypt. de Fr. n ${ }^{\circ}$ 1087).

In Herb.: Rolterdam: O udem. (v. vo et $s_{\text {。 }}$ ) - Bo. vendien: Maastricht, Franq.

3259. S. fragariae Desmaz, $\mathrm{O}_{\mathrm{p}}$ kwijuende bluden van Fragaria vesca. Aes!. Aut. 
Epiphylla, maculis suborbiculatis, fuscis, in ambilu brunneo-rubris. Peritheciis minutissimis, innato-promiuulis, fusco-fuligineis, ore orbiculari late apertis. Cirrhis albidis; sporidiis cylindricis, obtusis, curvatis vel rectis; sporulis 4 , oblongis hyalinis. (Desmaz. Not. sur 16 esps du genre Septoria, in Mém. Soc. Roy. des Sc. de Lille, $1842, \mathrm{n}^{\circ} 15$, p. 11 ; Depazea fragariaecola Wallr., Rabenh..p. 138).

In Herb.: Leiden, Dz. et $\mathbf{I I}$ b. (v. s.)

3290. 5. Gei Rob. Op treurende bladen van Geum ur. banum. Acst. Aut. (Rob, in Desmaz. Nouv, Not. Crypt, inéd. de Fr. in Ilém. Soc, Roy, des Sc. de Lille Mars 1843. p. 25. $\mathrm{n}^{\circ} 12$; Sphaeria (Depazea) vagans b. Geicola Fr. Syst. II. p. 532; Rabenh. p. 139).

In Ilerb.: Zuid-Beveland, vo d. B. (v. vo et s.) Bovendien: Maastricht, Franq.

3291. 5. Hederae Desmaz. 0 p de bladen van Hedera helix. Aut, Vere. (Desmaz, Nouv. Not. Crypt. inéd. de Fr. in Mém. Soc. Roy. Sc. de Lille. Mars 1843, no 9 pag. 24; Sphaeria (Depazea) hederaecola Fr. Syst. II. p. 528; Rabenh. p. 136; Dz. et II b. Bijdr. \%. p. 21).

In Herb.: Leiden, Dz, et $\mathrm{Nl}$ b. ; Maastricht, Fran q. (v. s.)

3292. F. Heterochroa Desmaz. Op treurende bladen van Malva-, Lamium-, Antirrhinum- en Plantagosoorten. Aest. Aut.

Maculis amphigenis, sparsis, minutis, suborbiculatis, griseo-brunneis dein albis evanidis, saepe brunneo cinc1is. Peritheciis parvis, epiphyllis, rarius hypophyllis, exilibus, fulvis, brunneis demum nigris, poro apertis, siccis concavis. Sporidiis linearibus tenuissimis, rectis vel curvulis. (Desmaz. 14 Not. Crypt. nouv, de Fr. p. 21. no 27), Variat: 
a. Planlaginis Desmaz. Op de hladen van Plantago major. Aut. (Desmaz. Pl. Crypt. de Fr. n 2172; Desmaz. $21^{\mathrm{e}}$ Not. Crypt. de Fr. p. 2. $\mathrm{n}^{0} 3$ ).

In Herb.: Kampen, R. B ond. (v. s.)

3293. \$. hydrocotyle Desmaz. $\quad 0_{p}$ treurende bladen van Hydrocotyle vulgaris. Aest. Aut.

Epiphylla; maculis irregularibus, rufis vel fuliggineis, dein exaridis; peritheciis minutissimis innatis, ore orbiculari apertis; cirrhis albidis; sporidiis linearibus, curvatis; sporulis 8-10 globosis, opacis. (Desmaz. Pl. Crypt. de Fr. no 1175; Desmaz. 9e Not. Crypt. inéd. de Fr. pag̣, 19).

In Herb.: Zuid-Beveland, v. d. B. (

3294. Incondita $R o b, O_{p}$ treurende bladen van Acer-, Quercus en Castanea-soorten. Aut.

Maculis amphigenis, irregularibus, rufis aut brunneoterreis, non limitatis, dein confluentibus. Peritheciis hypophyllis, minulissimis, numerosis, rufo-olivaceis, dein intense-brunneis, innato-prominulis, poro apertis, dein pauciusculis. Cirrhis albidis aut dilute carneis; sporidiis elongatis, tenuissimis, rectis vel curvatis, saepe obtusis, sporulis 4, cylindricis. (Desmaz. $21^{\mathrm{e}}$ Not. Sept. nouv, de la Fr. p. 11. no 20.) Variat:

a. Quercicola Desmaz. Il. et Pl. Crypt. de Fr. ${ }^{\circ}$. 2192. Op Eikenbladen.

In Herb : Leiden, v. d. B. (v. v. et s.)

3295. ฐ. leguminam Desmaz. Op de peulkleppen van Boonen en Erwten. Aut.

Peritheciis innato-prominulis, fulvo-brunneis, minulissimis numerosis, dense sparsis, vel concentricis, quandoque maculaeformibus poro apertis. Cirrhis carneis; sporidiis oblongis, obtusis; sporulis $2-4$ perexiguis, globosis, hyalinis. (Desmaz. Nouv. Not. 
Crypt. inéd. de Fr. in Mém. Soe. Roy. des Sc. de Lille, Mars 1843. p. 28. $n^{\circ}$. 15) Variat:

a. Pisorum Desmaz. II. $0 p$ de peulkleppen van Erwten (Pisum sativum.) Aut. (Ascochyta Pisi Lib.; Rabe n h. p. 146)

Maastricht, Franq. (n. v.)

3296. \$. mebulosa Desmaz. Op de stengels van Foeniculum vulgare Aut.

Erumpens. Maculis griseis, effusis vel elongatis. Peritheciis minutissimis, nigris, numerosissimis, dense sparsis, vel in series longissimas parallelas aggregatis; ostiolo simplici pertusis. Cirrhis tenellis albis; sporidiis linearibus, rectis vel carvulis; sporulis 10-15, perexilibus, opacis. (D esmaz. Nouv. Not. Crypt. inéd. de. Fr. in Mém. Soc. Roy. des Sc. de Lille, Mars 1843. p. 24. $n^{\circ}$. 10; Desmaz. Pl. Crypt. de Fr. no. 1331) In Herb.: Warmond, de Il a a n in Herb. de Vr. (v. v. et s.)

3298. 5. venotherae $W$ est. Op de bladen van Oenothera biennis. Aest. Aut. (West. Ilerb. Cr. B. $n^{\circ}$. 1152; West. 5e Not. Crypt. nouv. de la Belg. p. 32. $\mathrm{n}^{\circ} .96$; Depazea Oenotherae Lasch. in Raben h. p. 138 ?)

In Herb.: Amsterdam, v. d. S. Lac. (v. v. et S.)

3298. 5. orchidearum W est. Op de bladen van Orchis maculata en latifolia. Aest.

Taches d'un pâle brunâtre, arrondies, non limitées par des lignes plus foncées, de 5 à 10 millim. de diamètre, offrant au centre un grand nombre de périthèces très petits, d'abord d'un jaune d'ambre, devenant ensuite d'un brun foncé. Ostiole simple, poriforme. Cirrhe blanchâtre. Sporidies linéaires, atténuées anx extrémités droites ou légèrement flexueuses, contenant de 6 à 8 sporules globuleuses et transparanies, très difliciles à apercevoir. (West, $2^{e}$ Noto 
Crypt. nour. de la Belg. p.16. u. 24; West. Ilerb. Cr. B. $n^{\circ}$. 638)

In Herb.: Leiden, $\mathbf{D} z$, et $\mathrm{H}$ b. (v. v. et $\mathrm{s}$.)

3299. 5. oryacanthae $\mathrm{Kze}$ et $\mathrm{Sch} \mathrm{m}$. Op de bladen van Crataegus oxyacantha. Aut. (Kze. Myc. Hefte II. p. 109 ; D z. et II b., Bijdr. I. p. 30; Phlaeospora oxyacanthae Wallr. Comp. Fl. Germ. IV. p. 177; F r. Elench. Fung. II. p. 119)

In Herb.: Maastricht, Fran q.; Zuid-Beveland, v. d. B. (v. v. et s.)

3300. \$. populi Desmaz. $\mathrm{O}_{\mathrm{P}}$ treurende bladen van $\boldsymbol{P}_{0}$ pulus fastigiata. Aut. (Desmaz. Pi. Crypt. de Fr. $\mathrm{n}^{\circ}$. 1731; Sphaeria (Depazea) frondicola $\beta$ maculis minoribus Fr. Syst. Il. p. $529 ; \mathrm{D}$ z. et $\mathrm{II}$ b. Bijdr. I. p. 21)

In Ilerb.: Leiden, D z. et MI b.; Maastricht, Franq. (v. s.)

3301. 5. psevdoplatani $\mathrm{R}$ ob. $O p$ levende bladen van Acer pseudo-platanus. Aut.

Haculis amphigenis, minutissimis, sparsis, suborbiculatis, rufis, in ambitu luteolis. Peritheciis epiphyllis, paucis, conglomeratis, brunneis, convexis, dein collabescendo concavis. Cirrhis albidis. sporidiis linea:ibus, tenuissimis, curvulis. Sporalis 4 ? (Rob. in

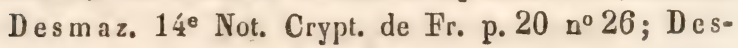
maz. Pl. Crypt. de Fr. $\mathrm{n}^{\circ}$ 1722).

In Herb.: Kampen, R. Bond. (v. v. et s.)

3302, \$. pyyi West. Op levende bladen van Pyrus communis. Aest. Aut.

Taches épiphylles, petites, éparses, arrondies ou anguleuses, brun-bistrées, circonscrites par une ligne plus foncée, formée par les nervures de la feuille. Au centre de chaque tache on remarque une autre plus petite, blanche ou jaune brunâtre, sur laquelle on voit les périthèces 
au nombre de 3 à 10 , très petits, épars, noirâtres, et perforés d'une ouverture simple, assez grande; cirrhe hlanc verdâtre. Sporidies allongées, linéaires, amincies aux extrémités, plus ou moins flexueuses et contenant de 8 a 12 sporules globuleuses, transparentes. (W e st. Ilerb. Cr. Belge $\mathrm{n}^{\circ} 136$; Depazea pyricola Desmaz.) In Herb.: Goes, v. d. B. (v. vo et s.)

3303. \$. quercina Desmaz, 0 p stervende Eikenbladen: (Desmaz. 14e Not. Crypt. de Fr. pag. 25, no 35; Depazea quercicola Wallr.; Rabenh. I. p. 137).

In Herb. : Leiden, D z. et $\mathrm{HI}$ b.; Amsterdam, v. d. S. Lac. ( . v. et s.) - Bovendien: Maastricht, Franq.

3304. Tosae Desmaz. Op treurende Rozenbladen. Aest. Aut.

Taches d'abord purpurines, puis brunes, arrondies, plus ou moins grandes, quelquefois entourées d'une aréole jaunâtre ou pourprée. Périthèces innés, bruns, épars, s'ouvrant au sommet par un pore arrondi. Cirrhe blanc. Sporidies linéaires, plus ou moins courbées contenant 8 à 12 sporales, globuleuses, opaques. (W est.

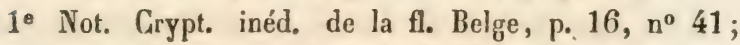
Desmaz. Pl. Crypt. de Fr. $n^{\circ}$ 535; Ascochyta rosaram Lib.; Rahenh. p. 145).

In Ilerb.: Zuid-Beveland, v. d. B. (v. v. et s.)

3305. 5. Tubl West. Op kwijnende Rubusbladen. Aut. (West. Ilerb. Cr. Belge $\mathrm{n}^{\circ}$ 938; Sphaeria rubi Duby. Bot. Gall. II. p. 712?; Depazea rubi West. et v. H a es. Cat. Crypt. no 80).

In Herb.: Maastricht, Franq. (v. vo et s.)

3306. \$. salicariae $W$ est. Op treurende bladen van Lythrum salicaria. Aut. (Ascochyta salicariae Franq. in litt.)

Ascis fusiformibus, tenuissimis, pellucidis. (Fran q. 1. I.)

Maastricht, Franq. (n. v.) 
3308. 5. Scabiosaceola Desmaz: $0 p$ levende bladen van Scabiosa succisa. Aest. Aut. (D esmaz. PI. Crypt. de Fr. $n^{\circ} 722$; Sphaeria (Depazea) vagans f. scabiosaecola Dec.; Fr. Syst. II. p. 532; R abenh. p. 139 ; Dz. et II. Bijdr. II. p. 17).

In Herb.: Utrecht, v. d. S. Lac. (v. s.) - Boven. dien: Maastricht, Franq.

3305. S. stachydis Rob. $O_{p}$ bladen van Slachys sylvatica. Aest. Aut.

Maculis amphigenis, subolivaceis, dein pallide brunneis sub-exaridis, irregularibus, venulis circtis, sparsis vel confluentibus. Peritheciis epiphyllis, minutissimis, brunneo-nigris, poro apertis. Sporidiis linearibus, tenuissimis, curvulis vel flexuosis; sporulis vix distinctis. (Desmaz. 14e Not. Crypt. de Fr. p. 19. n²2).

In Herb.: Utrecht, v. d. S. Lac. (v. v. et s.)

3309. S. diliae West. 0 p levende bladen van Tilia europaea. Aut.

Taches amphigènes, très petites (1 à 2 millim.), arrondies, éparses, d'un brun foncé presque noir, palissant un peu au centre, ou se trouvent éparpillés quelques périthèces rares, fort petits et noirs. Sporidies cylindriques, droites, souvent un peu plus grosses d'un coté, de 1 a $2 / 100^{\text {es }}$ de millim. de longuear, sur $1 / 400$ de millim. de largeur et offrant de 3 à 5 cloisons. (West. 5e Not. Crypt. de Belg. pag. $34 \mathrm{n}^{\circ} 108$; West. Herb. Cr. Belg. $\mathrm{n}^{\circ}$ 956).

Maastricht, Fran q. (n. v.)

Obs. Cette plante, a l'état stérile ou de vétusté est l'Ectostroma Tiliae des auteurs.

æ310. S. tomentillae Rob. Op kwijnende bladen van Potentilla-soorten. Aest. Aut.

Maculis amphigenis, minutis, ovalibus vel irregularibus, albidis exaridis, saepe roseo cinctis. Peritheciis epiphyllis, minutissimis, paucis, fuscis, poro anertis, 
sporidiis tenuissinis, flexuosis. (Desmaz. 14e Not. Crypt: de Fr. p. $22 \mathrm{n}^{\circ}$ 29). Variat:

a. Potentillae West. Op de bladen van Potentilla reptans. Aut. (West. $3^{e}$ Not. Crypt. inéd. de la FI. B. p. 17. $\left.n^{0} \cdot 67\right)$

In Herb.: 'Tjepma. (vos.)

3311. T. tussilaginis West. $O p$ kwijnende bladen van Tussilago farfara. Aut.

Taches nombreuses, éparses on confluentes, arrondies, irrégulières ou anguleuses, dépassant rarement 4 millim. de diamètre, d'abord brunes, puis devenant blanchâtres ou grisâtres, limitées par un bord épaissi et entourées d'une aréole pourprée. Périthèces tıès petits, immergés, éparpillés sur toute la tâche, noirâtres, à ostiole poriforme. Sporidies petites, flexueuses, cylindriques, tronquées aux estrémités, contenant de 8 a 12 sporules globuleuses et transparantes. (West. 2e Not. Crypt. inéd. de la Fl. B. p, 18. $\mathrm{n}^{\circ}$. 83)

In Herb.: Zuid-Beveland, v. d. B. (v. v. et s.)

3312. ๑. ulmi Fr. Op stervende bladen van Ulmus campestris. Aut.-Vere. (Fr. Nov. Fl. Suec. V. p. 78; Rabenh. p. 43; Dz. et Ml b. Bijdr. I. p. 30)

In Herb.: Zuid-Beveland, vo d. B.; Kampen, R. Bond.-Bovendien: Maastricht, Franq. (v. v. et s.)

\section{HHLTCRENA Desmaz.}

Perithecium spurium, convexum, ab epidermide nigुrifacta formatum, poro pertusum. Nucleus gelatinosus. Asci nulli. Basidia brevissima. Sporidiis curvatis elongatis vel fusiformibus dein ejectis. (Desmaz. $14 \mathrm{e}$ Not. Crypt. de Fr. p. 16)

3313. Ph. vagabunda $\mathrm{Desmaz}$. $0 p$ doode stengels van kruidgewassen. Vere, Aest. 
Iflaculis nullis vel minutissimis, brunneis, saepe fio hrillosis. Pseudoperitheciis numerosis, sparsis, exilissimis. Sporidiis hyalinis, curvatis, elongatis, linearibus, utrinque subobtusis. Sporulis 7-9, vix distinctis. (Desmaz. II. p. 16. no. 16; Ascochyta caulium. Lib. Pl. Crypt. Arủ. no. 248)

$$
\text { In IIerb.: Ilaastricht, Franq. (v. s.) }
$$

\section{WERMCULARA $\mathrm{Fr}$.}

Perithecium superficiali-adnatum, pilis setosis erectis vestitum, astomum, demum basi secedens. Sporidia fusiformia septata (Fr. Summa Verg. Scandin. p. 419)

\section{* Ca alicolac.}

3314. v. chemoporioi West. Op doode stengels van Chenopodium album. Hieme-Vere.

Périthèces d'un noir brunâtre, saillants, arrondis, très petits; épars, innés, couverts de poils rares, courts, très fragiles, brunâtres. Ostiole irrégulier. Sporidies hyalines, fusiformes, légèrement courbées en croissant, de $1 / 100^{\circ}$ de mill. de longueur sur $1 / 400^{\circ}$ de mill. de largeur et offrant souvent une cloison au milieu. (West. 5e Not. Crypt. inéd. de la FI. B. p. 23. no. 48; West. Herb. Cr. Belge $n^{\circ}$. 1233)

In Herb.: Amsterdam, v. d. S. Lac. (v. v. et s.)

3315. W. dematium $\mathrm{Fr}$. Of doode stengels van groote kruidgewassen. Vere. (Fr. Summ. Veg. Scand. p. 420 ; Sphaeria dematium Pers.; Rabenh. I. p. 195; Dz. et $\mathrm{Ml}$ b. B̈̈jdr. I. p. 20)

In Herb.: Leiden, Dz. et $\mathbb{M} b$; W $\mathrm{t} t \mathrm{w} ;$; Utrecht ; v. d. S. Lac. - Bovendien: Maastricht, Franq.

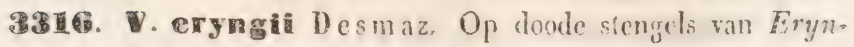


gium campestre. Hieme. Vere. (Desmaz. Pl. Crypt. de Fr. $\mathrm{n}^{\circ}$. 542; Exosporium eryngii Pers.; Helminthosporium eryngii Fr. Syst. III. p. 361).

In Herb.: Noordwijk, D z. et II b.; Harderwijk, Jungh. in Ilerb. de Vr.; Kampen, R. Bond.; ZuidBeveland, v. d. B. (vo vo et s.)

331 8 . Werbarum $W$ est. Op doode stengels van kleine kruidgewassen. Vere.

Périthèces innés, épars, très petits, arrondis ou ovalaires, surmontés d'un faisceau de poils bruns (plus pâles et transparents vers le sommet), raides et acerés, et s'ouvrant au sommet par une déchirure inégale. Sporidies allongées, atténuées et courbées aux extrémités, ayant $1 / 50^{e}$ à $1 / 60^{\circ}$ de mill. de longueur, contenant de 3 à 5 sporules globuleuses et transparentes. (West. 2e Not. Cr. inéd. de la B. p. 29. no. 146) Variat :

a. Dianthi West. 1. I. Op doode stengels van Dianthus superbus. In de hoven.

In Herb.: Zuid-Beveland (Goes), v. d. B. (v. vo et s.)

b. Sedi West. Op doode stengels van Sedum telephium. Vere. (West. 4e Not. Cr. inéd. Belgg. p. 12. nº 49)

In Herbr: Zuid-Beveland (Goes), v. d. B. (v. v. et s.)

3315. V. liliacearum West. $O p$ doode bloemstengels van Ornithogalum, Scilla en Allium-soorten. Hieme. Vere.

Périthèces très petits, noirs, luisants, arrondis ou ovalaires, convexes, épars', surmontés d'un faisceau de poils assez fins, d'un noir brunâtre, variant pour sa longueur entre 3 et $9 / 100^{\mathrm{e}}$ de mill. et caducs dans un âge avancé. Ostiole poriforme ou irrégulier. Sporidies hyalines, cylindriques, atténuées aux extrémités, plus ou moins courbées en forme de croissant, longues de $1 / 50^{\mathrm{e}}$ de mill. sur $1 / 200^{\mathrm{e}}$ de mill. de largear et of- 
frant souvent une eloison an milien. (West, 5e Not. Cr. inéd. de la Fl. B. p. 23, no. 47)

In Herb.: Amsterdam, v. d. S. L a c. (v. s.)

3319. V. maculans Desmaz. Op doode Aardappelstruiken. Ilieme. Vere. (Desmaz. P1. Crypt. de Fr. no. 339; Fr. Summ. Veg. Scand. p. 420; Exosporium maculans Link.)

In Herb.: Naaldwijk, v. d. Tr. (v. v. et s.)

3320. V. minutum Desmaz, $O_{p}$ doode stengels van kruidgewassen. Hieme, Vere. (Desmaz. Pl. Crypt. de Fr. no. 231 ; Sphaeria dematium $b$. minor Fr. Syst. II. p. 505 ; ? Sph. minuta Rabenh. I. p. 196)

In Herb.: Leiden, $\mathrm{D} x_{\text {. }}$ et II b.; Utrecht, Splitg. in Herb. de Vr. (v. v. et s.) Variat:

a. Hederae West. in Herb. Op stervende bladen van Hedera helix. (Exosporium minutum V Ilederae Desmaz. Pl. Cr. de Fr. n ${ }^{\circ}$ 1848)

In Herb.: Leiden, v. d. B. (v. v. et s.)

\section{* *}

3321. V. trichella $\mathrm{Fr}$. $0 \mathrm{p}$ doode bladen van Hedera helix. Hieme. Vere. (F r. Sumın. Veg. Scand. p. 420; Sphaeria trichella Fr. Syst. II. p. 515; Rabenh. p. 195)

In Ilerb.: Zuid-Beveland (Goes), v. d. B.; Utreeht, v. d. S. Lac. (vo vo et s.)

3322. W. hispidula West. Op bladen, halmen en aren van afgesneden grasgewassen. Aut. Hieme. (Exosporium hispidulum Link.; Rabenh. p. 33)

Maastricht, Fran q. (n. v.)

3a23. $v$. depazoldes $W$ e $3 t$. in Herb. Op treurende bladen van de Vlier. Aut. (Exosporium depazoides Desmaz. 17" Not. Cr. de Fr. p. 38. $n^{\circ} .56$ ) 
Amphigenum, numerosum, maculae albae insidens. Stromate punctiformi. Setis brevibus, fusco-nigris, subdivergentibus. Sporidiis rarissimis, clavatis, septatis , semihyalinis. (Desmaz. 1. l.)

In Herb.: Kampen, R. Bond. (v. s.)

\section{Trib. Sporophorac.}

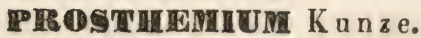

3321. PPr. betulinum $K$ unze. $O p$ de schors van aigestorven takken van Betula alba. Hieme. Vere. (Fr. Syst. III. p. 484; Rabenh. p. 142)

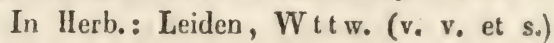

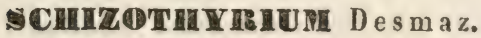

Perithecium sessile, simplex, subcorneum, rolundatum vel ovatum, applanatum vel convexiusculum, minutum, punctiforme, rima lonģitadinali dehiscens; nucleo gelatinoso, albido. Asci fisi, erecti. Sporidiis ovoideis simplicibus vel compositis. (Desmaz. 17e Not. Cr. de la Fr. p. 34)

3325. \$. ptarmicae Desmaz. Op levende stengels en bladen van Achillea ptarmica in de lusthoven. Aest. Aut. (Desmaz. I. I. p. 35. $\mathrm{n}^{\circ} .50$; Labrella ptarmicae Desmaz. Pl. Cr. de Fr. no. 189; Dz. et Mb. Bijdr. I. p. 22; Fr. Elench. fung. II. p. 149)

$$
\text { In Herb.: Leiden, Dz. et M b. (v. v. et s.) }
$$

\section{ACTINOTHEYR HUT $\mathrm{K} z \mathrm{e}$.}

3326. Araminis $\mathrm{K} z \mathrm{e} . \mathrm{O}_{\mathrm{p}}$ doode grashalmen. Aut. Vere. (Fr. Syst. II. p. 597; R abenh. p. 142) In Herb.: Rotterdam, v. d. B. (v. v, et : s。) 


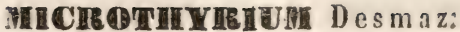

Perithecium simplex, superficiale, membranaceum, adpressum, scutiforme, centro perforatum, obtegens ascos fixos subclavatos. (D esmaz. 8e Not. Crypt. de Fr. p. 9. PI. XIV. fig. 1)

3328. MI. microscopicum Desmaz. Op doode en afgevallen bladen van Ilex aquifolium. Aut.

Epipbyllum. Maculis magnis, irregularibus, fuscocinerascentibus; peritheciis sparsis, minutissimis, tenuissimis, nigris, subuitidis, papillatis; ascis clavatis; sporidiis septatis, oblongis, subfusiformibus. (Desmaz. 1. 1. p. 10; Desmaz. Pl. Crypt. de Fr. n. 1092)

In Herb.: Leiden, $\mathbf{D}$ z. et $\mathbb{I}$ b. (v. v. et 8 .)

Obs. Cette espèce est souvent mêlé sur les mêmes feuilles avec le Phacidium multivalve et l'Eustegia ilicis.

CETPSTHEMA Fries.

3328. L. Scirpi $R$ abenh. $O_{p}$ doode halmen van Scir. pus lacustris. Hieme. Vere. (R a benh. I. p. 140; Dz. et II b. Bijdr. II. p. 17)

In Herb.: Zuid-Beveland, vo d. B. (v. v. et s.)

3329. W. juncinum $\mathrm{Fr} . \mathrm{O}_{\mathrm{p}}$ doode halmen van Juncrs conglomeratus. Hieme. Vere. (Fr. Syst. II. p. 598; Rabenh. p. $140 ; D z$. et II b. Bijdr. II. p. 17)

In Ilerb.: Zuid-Beveland (Goes), v. d. B. (v. v. et s)

3330. L. filicinum Fr. $O_{p}$ doode loofstelen van Pteris nquilina. Aut. (F r. Syst. Il. p. 599; R a ben h. p. 141; Dz. et $\mathrm{Mb}$. Bijdr. II. p. 18)

In Herh.: Haarlem, Dz. et II ho; Haastricht, Franrq. (v, v. et s.)

3331. L. Litigiosam Desmaz, Op doode loofstelen van Pteris aquilina. Aut. Hieme. 
Perithecium subrotundum, minutissinum, punetiforme, sparsum vel conglomeratum, brunneo-nigrum, demum totum secedens. (Desmaz. Ilém. de la Soc. Roy. des Sc. de Lille. MIars 1843. pag. 21. no. 5; Desmaz. Pl. Cr. de Fr. no ${ }^{0}$ 1327)

In Haarlem, $\mathbf{D}$ z. et $\mathrm{MI}$ b. (v. v. et s.)

3332. I. vulgare Fr. $0 p$ doode stengels van verschillende kruidgewassen. Aut. Hieme. (F r. Syst, II. p. 599; Rabenh. p. 141)

In Herb.: bij Zutphen, West. ex herb. G. A u b. (v. v. et s.) Variat:

a. Verbenae R abenh. (West. Ilerb. Cr. Belg. $\mathrm{n}^{\circ}$. 1246). $\mathrm{O}_{\mathrm{p}}$ doode stengels van Verbena offic.

In Herb.: Zuid-Beveland, v. d. B. (v. vo et s.)

3333. I. seirpinum Fr. Op doode halmen van Scirpus lacustris. Aut. Hieme. (F r. Syst. II. p. 598; R abenh. p. 140)

In Herb.: Hulst, West. (v. v. et s.)

Obs. Ne pas confondre avec le $L$. scirpi qui croit sur les mêmes plantes.

3331. L. ocellatum Pers. $0 p$ doode bladen van Betula alba. Hieme. (Pers. in Herb.; Dz. et Il b. Bijdr. I. p. 22)

In Herb.: Leiden, Dz, et $\mathbf{I I}$ b. (v. s.) An Leptothyrium betulae Lib.?

\section{LEFTOTIIIIRUNI $\mathrm{Kun} z$.}

3335. T. juglandis Lib. $O_{p}$ treurende en stervende bladen van Juglans regia. Aut. (Lib. Pl. Crypt. Ard. n. 164; Sphaeria (Depazea) jugrlandicola Fr. Elench. fung. II. p. 110; Depazea juglandina $\mathbf{h a -}$ ben h. p. 137)

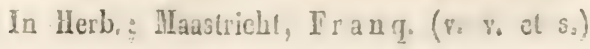


3336. L. populi Lib. Op stervende bladen van Populus nigra en fastigiata. Aut.

Epiphyllum. Maculis primo orbiculatis, subradiatis, demum irregularibus confluentibus fuscis; peritheciis planiusculis, pallidis, basi circumscissis; pulpa alba; ascellis subclavatis; sporidiis 3.4 pellacidis. (Lib. PI. Cr. Ard. $n^{0} .257$ )

In Ilerb.: Busloo bij Zutphen, West, ex herb. G. A ub. (v. v. et s.)

333\%. H. ribis $L i b . O_{p}$ treurende bladen van roode Aalbeziën. Aut.

Epiphyllum. Mlaculis orbiculatis, minutis, confluentibus, fuscis; peritheciis planiusculis rufo-gilvis, basi circumscissis; pulpa alba; ascellis ovatis, curvatis; sporidiis 3 pellucidis. (Lib. Pl. Crypt. Ard. no. 258)

In Herb.: Kampen, R. B ond.; Leiden, D z. et II b. ; Zuid-Beveland (Goes), v, d. B. (v. v. et s.)

\section{Trib. Asporae.}

\section{ATEm DM Dec.}

3338. A. atratum $\mathrm{Chev}, 0 p$ de bladen van Scabiosa. Aut.

Epiphyllum anguloso-subrotundum aterrimum confluens late effusum, peritheciis minimis confertis. (Chev. Fil. Par. I. p. 449)

Maastricht, Franq. (n. r.)

3339. A. betulae Rob. $0 p$ rottende bladen van Betula alba. Hieme. Vere.

Amphigena, maculaeformis. Fibrillis innatis, brunneis, ramosis e centro radiantibus; ramis numerosis, apice dirergentibus. Peritheciis erumpentibus, sparsis, minutissimis, nigris. (Rob. in Desmaz. Mém. Sore 
Ihoy. des Sc. de Lille. Mars 1843 p. 36. 10 22; Desmaz. PI. Cr. de Fr. no 1346.

$$
\text { In Herb.: Hulst, W est. (v. v. et s.) }
$$

3340. A. delicatulum Desmaz. $0_{p}$ de zadhuisjes van Lunularia rediviva. Ant.

Maculis minutis, orbiculatis, quandoque coufluentibus, humidis subolivaceis, siccis griseo-nigris. Fibrillis innatis tenerrimis flexuosis, c centro radiantibus. Ramis paucis, elongatis subdichotomis, articulatis; articulis diametro aequalibus. Peritheciis minutissimis, sparsis, globosis. (Desmaz. 19e Not. Crypt. de Fr. p. 8; Desmaz. Pl. Cr. de fr. 1993.)

In Herb.: Maastricht, Fran q. (v. s.)

3341. A. graminis West. Op rottende halmen van verschillende grassoorten. Aut. Vere.

Rosettes très petites, arrondies ou allongées, d'abord éparses puis confluentes, d'un gris olivâtre, formées par des fibrilles superficielles, divergentes, rameuses, très déliées et fort nombreuses. Périthèces excessivements petits, noirs, luisants, éparpillés sans ordre sur toute la rosette. Sporidies inconnues. (West. $5^{\circ}$ Not. Crypt. inéd. de la fl. B. p. 27, $\mathrm{n}^{\circ}$ 73.) Variat:

a. Ammophilae West. I. I. $0 p$ de halmen en bladen van Ammophila arenaria. (W est. Herb. Cr. Belge $n^{\circ}$ 1051.)

In Ilerb.: de Hollandsche duinen, 0 udem. (v. v. et s.)

3313. A. popali Rob. Op rottende Populierbladen. Hieme. Epiphylla. Maculis nullis vel albidis, minutis, suborbiculatis, numerosis, saepe confluentibus, fibrillis tenuissimis, brevibus, densis, ramosis fusco-nigris e centro radiantibus. Peritheciis iggnotis. (Desmaz. 14 Nuf, Crypt. de Fr. p. $\left.35, n^{\circ} 54.\right)$

In Herb.: Utrecht, vo d. S. Lac. (v, s.) 
3343. M. rosae Lib. $O p$ kwijnende Rozenbladen. Aut. (Lib. Ann. Soc. L. Par. V.p. 405; Erysiphe radiosum Fr. Syst. IlI. p. 247; Sporotrichum maculare Link.; Ast. radiosum Fr. El. II. p. 151; Rabenh. p. 139.)

In Herb.: Leiden, Dz. et II b.; Amsterdam, vo d. S. La c.; Goes, v. d. B.; Maastricht, Franq. (v.v. et s.)

3341. A. ulmi $C$ h e v. $O_{p}$ stervende Olmbladen. Aut. Hieme.

Epiphyllum. Subrotundum fibrillis, centro tandem opaco, divaricatis. Peritheciis parum conspicuis innatis. (Chev. fl. Par. I. p. 448; Dothidea ulmi Grev。?)

In Ilerb, : Zalk, R. Bond. (v. s.)

\section{ECTOSTTERA $\mathrm{Fr}$.}

3345. E. lixiodendri $\mathrm{Fr}$. Op treurende bladen van $\boldsymbol{L} y$ riodendron tulipiferum. Aut. (F 1 . Syst. 1I. p. 602; Dz. et II b. Bịdr. I. p. 23; Lep!ostroma lyriodendri Link.; Rabenh. p. 141.)

In Herb.: Leiden, in den Hortus Academicus, Dz. et $M I$ b. (v. vo et s.)

3346. F. sedli $\mathrm{Fr}$. Op stervende stengels en bladen van Sedum telephium. Aut. (F r. Syst. II. p. $602 ; \mathrm{D}$ z. et II b. Bijdr. I. p. 22; Leptostroma sedi Link; Rabenh. p. 142.)

In Herb. : Leiden, $\mathbf{D}_{\text {z. et }} \mathrm{M}$ b。; $\mathbf{O} \mathrm{udem}$. (v. vo et s.)

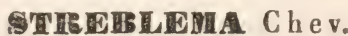

Fragile expansum submembranaceum totum nigrum intra ligni putridi fibras retroflexas efficiens. ( $C h \in \mathbf{v}$. ก. Par. I. p. 508).

334 8. 5. entogenum $\mathrm{G} h \mathrm{e}$, Tusschen de vezels van rottend hout, in holle Wilgenstammen, enz. Per annum.

Sinubus nigris crassiusculis late vageque serpentibus interdum ligno resecto duplicatis, passim ramosis anostomosantibus. (Che v. Il。 p. 508.)

In Herb.: Zuid-Beveland, v. d. B. (v. s.) 


\section{Drd. IInplieomyceles.}

* II ucorini.

Trib. Pilobolei Corda.

PPILOBELUS Tode:

3345. Cristallinus Tode. $0_{p}$ r koemest, bij vochtig weder. Per annum. (Fr. Syst. III. 312; Rabenh. p. 135.)

In Herb.: Zuid-Beveland, v. d. B. - Bovendien; Naaldwijk, v. d. Tr. (v. vo et Icon.)

\section{Trib. Illucoroideac.}

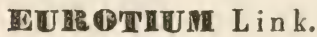

3349. E. Herbariorum Link. Op slecht gectroogde planten in Herbaria: Per annum. (Fr. Syst. III. p. 332; R abenh. p. 133; Dz. et II b. Bijdr. I. p. 28.)

In Herb.: Leiden, Oudem. (v. vo et s.) Variat: a. epixylon $\mathrm{K} z$ e et $\mathrm{Sch}$. Op vochtig hout in de kelders. (R a benh. p. 133; Dz. et Hil b. Bijdr. II. p. 19) In Herb.: Leiden, Oudem.; Dz: et II b, ; ZuidBeveland, $v, d$, B. ( $v$, v. et s.) 
IHUCUIC Mieh.

3350. H. mucedo Linn. $O_{p}$ bedorsen spijzen, brood ete. Per annum. (Fr. Syst. III. p. 320; R abenh. p. 130; Dz. et $\mathrm{Hl}$ b. Bijdr. Il. p. 19)

In Herb.: Leiden, $\mathrm{D} z$. et $\mathrm{M}$ b.; Zuid-Beveland, v. d. B. (v. s.)

3351. MI. caninus Pers, $O_{p}$ hondsdrek op vochtige plaatsen, in kelders, enz. Per annum. (R a ben h. p. 130.)

In Herb.: Leiden, Dz. et II b. (v. v. et s.)

3352. Fn. macrocarpus Corda. $O_{p}$ den hoed en den vruchtdrager van Mycena galopus, Aut. (Corda Ic. fung. 1I. p. 21. T. XII. f. 84 ; R abenh. p. 131; D z. et $\mathrm{Il}$ b. Bijdr. II. p. 19)

In Herb.: 's Hage, G. B.

A.COPHOHA Tode.

3353. A. chalybaca $D_{2}$, et $I l b . O_{p}$ de lamellen van Agaricus rimosus. Aug. (Dz. et M b. Tijdsch. v. Nat. Gesch. en Physiol. XII. p. 283. T. VI. f. 4-10; Bijdr. II. p. 19)

In Herb.: Leiden, $\mathrm{Dz}_{\text {z. et }} \mathrm{II} \mathrm{b}$. (v. v. et Ic.)

3354. A. Iructicola Corda. Op roltende pruimen, aalbessen en andere vruchten, na regenachtig weder. Acst. (Corda Ic. fung. II. p. 20. T. XI. f. 82; Rabenh. p. $130 ; \mathrm{D}$ z. et MI b. Bijdr. II. p. 19)

In Herb.: Leiden, Dz. et M b.; Zuid-Beveland, v. d. B. (v. s.)

Trib. Igariae.

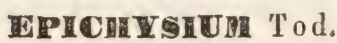

3355, E. argenteum Tode. Op rottenden koemest. Vere. Aest. (Fr. Syst. III. p. 293; Rabenh, p. 125; Tode Fung. Mleck. II. p. 1. T. VIII. f. 60.)

In Herb.: Zuid-Beveland (Gocs), $v_{0} d_{0}, B .(v, v, c t s$. 


\section{Trib. Stibini.}

STILBED Tode.

3356. 5. erythrocephalum Ditm. $O_{p}$ drek vill kunijnen, hazen, enz., bij vochtig weder. $\Lambda$ ut. (F r. Syst. III. p. 303 ; Rabenh. p. 122.)

In Herb.: Rotterdam, 0 udem. (v. v. et s.)

335\%. S. rigidum Pers. $\mathbf{O}_{\mathrm{p}}$ rottend hout. Per annum. (Fr. Syst. III. p. 303; R abenh. p. 122; Dz. et II b. Bijdr. III. p. 10)

Naaldwijk. v. d. Tr. (n. v.)

\section{PERTCDNIA Pers.}

3358. atra $\mathrm{C}$ orda. $\mathrm{O}_{\mathrm{p}}$ verdroogde stengels van Paconia officinalis. Aut. (Corda Ic. fung. I. p. 19. T. V. f. 258 ; Rabenh. p. 118.)

In Ilerb.: Leiden (Studentenpad), Dz. et II b. (v. v. et s.)

Trib. IRacodiei.

IBIBACUICLADIUM Corda.

3359. IB. penicillatum $\mathrm{C}$ orda. $\mathrm{O}_{\mathrm{p}}$ doode stengels van Papaver somniferum. Aut. (Cord. Ic. fung. II. P. 14. T. X. fig. 63 ; R a benh. p. 117)

In Herb.: Leiden, Dz. et MI b. (vo vo et s.)

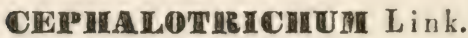

3360, C. Stemonitis $\mathrm{N}$ ees. Op rottend hout, alsook op rottende halmen van Phragmites communis. Aut. Vere. (F r. Syst. III. p. 280 ; Periconia stemonitis Per's. Rabenh. p. 118; Dz. et Mlb. Bijdr. I. p. 27)

In Herb.: Leiden, Dz. et II b. (v, s.) 


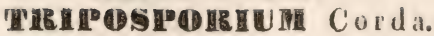

33361. T. elegans $\mathrm{C}_{\mathrm{o}} \mathrm{rda}$. Op rottende Berkentakken en -spanen. Aut. Vere. (Corda Ic, fung. I, p. 16. T. IV. fig. 220; Rabenh. p. 116)

In Ilerb.: Zuid-Beveland (Goes), v. d. B. (v. vo et s.)

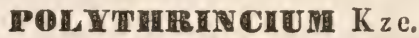

3362. EP. trifolii $\mathrm{Kz}$ e. $\mathrm{O}_{\mathrm{p}}$ treurende bladen van versehillende Klaversoorten. Aut. (Fr. Syst. III. p. 338; Rabenh. p. 115; Dz. et 11 b. Bijdr. I. p. 28)

In Ilerb.: Leiden, D z. et II b., O u dem.; ZuidBeveland, Zwake, v. d. B.; Zwijndrecht, v. d. S. L a c. (v. v. et s.)

Bovendien: Maastricht, Franq.

\section{CLATOSPOHEUTI L in}

3363. Cl. herbarum Link. $O p$ afgestorven stengels van kruidgewassen. Aut. Hieme. (F r. Syst. III. p. 370; Rabenh. p. 113; Dz. et Mlb. Bijdr. I. p. 28)

In Herb.: Leiden, D z. et II b.; Kampen, R. Bond. (v. s.)

3364. Cl. fuscum $\mathrm{N}$ ees. Op levende bladen van Artemisia vulgaris. Aut. (F r. Syst. III. p. 370 ; Rabenh. p. 113)

In Ilerb.: Ilaastricht, Franq.

3365. Cl. fumago Link. $O_{p}$ levende bladen van verschillende boomen en heesters. Aest. Aut. (F r. Syst. III. p. 370; Rabenh. p. 113; Dz. et II b. Bijdr. I. p. 28; Fumago vagans Per s.)

In Herb.:

c. Op Acer, Leiden, Dz. et $\mathbb{I I L}$.

b. Op Tilia, Lciden, 0 adem. 
c. Op Quercus, Leiden, $\mathbf{D}$ z. et II b:

d. Op Viburnum tinus, Zaid-Beveland (Goes), v. d. B. e. Op Pyrus malus, Goes, v. d. B.

f. Op Ribes nigrum, Leiden, $\mathrm{Dz}$. et $\mathrm{II}$ b. (v. v. et s.)

3366. Cl. epiphyllum Link. Op afgestorven Populierbladen. Aut. (Fr. Syst. III. p. 370; Rabenh. p. 113; Dz. et MI b. Bïjdr. III. p. 20)

In Herb.: Leiden, Dz. et $\mathrm{MI}$ b. ; Kampen, R. B on d. ; Zuid-Beveland, v. d. B. (v. v. et 8.)

Bovendien: Maastricht, Franq. (n. v.)

3368. C1. dendridicum $\mathrm{W}$ all $\mathrm{r}$. Op levende bladen van Appelen en Peren. Aut. (Rabenh. p. 113) Variat:

a. Pyri Desmaz. Pl. Cr. de Fr. ${ }^{\circ}$. 2137.

In Herb.: Maastricht, Franq. (n. v.)

3368. Cl. graminum Link. $O_{p}$ kwijnende bladen en halmen van verschillende Grassen. Aut, (R abenh. p. 113)

Mlaastricht, Franq. (n. vo)

3369. Cl. fascicullare $\mathrm{Fr}$. Op stervende stengels van kruidgewassen, alsook op de vruchten van Esschen. Aut. Hieme. (F r. Syst. III. p. 370; R a be nh. p. 113; Dz. et II b. Bijdr. III. p. 20)

In Herb.: Leiden, Dzo et II b.; Kampen, R. B ond.; Zuid-Beveland, v. d. B. (v. s.)

3380. Cl. fasciculatum Corda. $O p$ rottende halmen van Scirpus lacustris. Aut. (Corda Ic. fung. I. p. 15. T. IV. fig. 216; R a benh. p. 115)

In Herb.: Maastricht, Franq. (v. s.)

33z1. Cl. typharum $D$ esmaz. 0 p afgestorven bladen van Typha angustifolia. Aut. (Desmaz. Pl. Cr. de Fr. $n^{\circ}$. 304. Sine deser.)

In Ilerb. : Amsterdam, v. d. S. L a c. (v. v. et s.) 


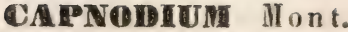

Peridium carnosum, varium, elavatum, lageniforme ant ceranoideun, apice irregulariter rumpens, e strato duplici formatum, exteriori scilicet colorato celluloso, cellulis inaequaliter penta-hexagonis, in ostiolo parallelogrammis linearibusque, interiori mucilagineo byalino fere anhisto. Nucleus gelatinosus bibulus. Asci late obovoideo-clavaeformes, mox diffluentes, sporas subsenas, oblongas, transversim septatas, tandem septis longitudinalibus accedentibus cellulosas, fuscescentes, includentes. Thallus nigrescens superficialis, lihere evolutus, e floccis brevibus contortis aut moniliformibus, ramosis articulatis fuscis dense intricatis compositus. (II on t. Ann. des Sc. Nat. 3e Sér. T. XI.p. 233; Mo nt. Syll. Gen. Spec. Crypt. p. 256.)

3382. C. Sphaeroideum Lacr. Op schors van Wilgentakken. Hieme. Vere. (Lacr. in Desmaz. Pl. Cr. de Fr. 2e Sér. n 769. Sine descr.; Rabenh. Fung. Eur. $\mathrm{n}^{\circ} 352$ )

In Hlerb.: Studentenpad bij Leiden, de II a a n in Herb. de Vr. (v. s.)

PASSALDRA IIont. et Fr.

Fibrae tenellae, flexunsae, subramosae. Sporidia longe stipitata, stipite articulo discreto, ceterum simplicia et primitus coneatenata. (II o n t. Ann. des Sc, nat. 2e Série T. VI. p. 30. T. 12 f. 4.)

3383. I. bacilligera II ont. et $\mathrm{Fr}$. $0 p$ levende Elzenbladen. Aut.

Hypophylla; fibris tenellis subramosis flexuosis, apice incrassato obtusis, olivaceis septatis intricatis, maculas minutas fuliginosas efformantibus; sporidiis simplicibas longe stipitatis; stipite articulo discreto, primitus con- 
catenatis, (II on t. I.I. pag. 31. T. XII. fig. 5; Mont. Syll. Gen. Spec. Crypt. p. 305)

In Herb.: Leiden, $\mathbf{D} z$. et $\mathrm{II} b$. (v. v. et s.)

\section{HELMINTHIOS PORIUPI $\mathrm{L}$ in $\mathrm{k}$.}

3384. Hit. velatinum $L i n k$. $O p$ doode takken van verschillende boomen en heestergewassen. Per annum. (Fr. Syst. III. p. 359; R a ben h. p. 108; Dz. et Mil b. Bijdr. III. p. 10)

In Herb.: Leiden, Dz, et II b. (v. v. et s.)

3385. 耳I. tenuigsimum $\mathrm{Nees}$. $O p$ rottende bladen van een Pancratium. Hieme. ( $\mathrm{R}$ abenh. p. 109; Macrosporium tenuissimum Fr. Syst. III. p. 374.)

Maastricht, Fran q. (n. vo)

3376. III. arundinaceum $\mathrm{C}$ orda. $\mathrm{Op}_{\mathrm{p}}$ stervende bladen van Phragmites communis. Aut. (C o r d a Ic. fung. III. p. 10. T. II. f. 25 ; R a benh. p. 109.)

In Herb: Zuid-Beveland, v. d. B.; Amsterdam, v. d. S. Lac. (v. v. et s.)

338\%. III. folliculatum $\mathrm{C}$ ord a, Op rottend hout, bijzonder van Corylus avellana, op rochtige plaatsen. Hieme. (Corda Ic. I. fung. p. 12. T. III. f. 180 ; $R$ a benh. p. 110.) Variat:

a. brevipilum Corda I.I. II. pag. 13. T. X. f. 60 ; R abenh. 1.I.

In Herb.: Zuid-Beveland (Goes), v. d. B. (v. s.)

3378. III. gracile $R$ abenh. $0 p$ sterrende bladen van Iris, Gladiolus, enz. (R a benh. p. 110.)

In Ilerb.: Rotterdam, $\mathrm{Oudem}$. (v. v. et s.)

3379. II. macrocarpum Grev. $O p$ afrestorven Elzentakken. Aut. Hieme. (Cord. Ic. fung. I. p. 12. T. II. f. 177: Rabenh. p. 110 ; Fr. Syst, III. pag. 356 ; Dz. et M h. Bịdr. III. p. 10) 
In Herb.: Haren bij Groningen, de II a a n; Leiden; Dz. et Ml b. ; Maastricht, Franq. (v. v. et s.)

3330. Hil. appendiculatum Corda. $0 p$ doode en afgevallen Esschentakken. Aut. Vere. (Cord. Ic. fung. : p. 12. T. III. f. 178 ; $R$ abenh. p. 111.)

In Ilerb.: Zuid-Beveland, v. d. B.; 't Haagsche Bosch en Blauwkappel, v. d. S. Lac. (v. s.)

3351. HI. scolicoides $\mathrm{C}$ orda. Op rottende koolstronken. Vere. (Corda Ic. fung. I. p. 12. T. III. fo, 179; Rabenh.p. 111.)

In Herb.: Leiden, W $t \mathrm{tw}$. (v. s.)

3352. 11. betulinum $\mathrm{C}$ orda. $O_{p}$ rottende schors van Betula alba. Aut. Vere. (Corda Ic. fung. I. p. 12. T. III. f. 184; R abenh.p. 111.)

In Herb.: Ilarderwijk, J ungh. in Ilerb. de Vr. (v, s.)

3393. 顺. Longipilum $\mathrm{C}$ orda. $\mathrm{O}_{\mathrm{p}}$ afgestorven Berkenschors. Per annum. (Corda Ic. fung. T. I. p. 14. III. f. 197; Rabenh. p. 112)

In Herb.: Harderwijk, Jungh. (vo. s.)

3384t. DI. Tasciculare $\mathrm{C}$ orda. Op rottend hout op wochtige plaatsen. Ilieme. (Gorda Ic. fung. I. p. 12. T. II. fig. 198; R a benh. p. 112)

In Ilerb.: Zuid-Beveland (Goes), vo d. B. (v, s.)

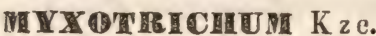

3355. ㅍ. chartarum $K z$ e. et $S c h m$. Op rottend grauw papier op vochtige plaatsen. Per annum. (Fr. Syst. III. p. 349 pro parte; Rabenh. p. 105; Dz. et HIb. Bijdr. I. p. 28; Oidium chartarum Link.)

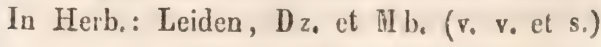




\section{AETHR日NUVI ( $K$ unze) Link.}

3356. A. Caricicola $\mathrm{Kunze}$ et $\mathrm{Sehm} . O_{p}$ stervende bladen van Carex riparia en andere Carex-soorten. Ant. (Fr. Syst. III. p. 377; Rabenh. p. 104)

In Herb.: Mniden, v. d. S. L a c.; Leiden, D z. et Mb. (v. v. et s.)

\section{CAPHPTOUNI $\mathrm{L}$ in $k$.}

3388. C. curvatum L k. 0 p bladen van groote Carex-soorten. Hieme-Vere. (F r. Syst. III. p. 377 ; R a ben h. p. 103) In Ilerb.: Amsterdam, v. d. S. Lac. (v. v, et s.)

\section{* II acedinei.}

\section{Trib. DHeedinei genuini.}

\section{VERTCILHUDI $N$ es.}

3359. V. tenerum Nees. $O_{p}$ rottend hout op vochlige plaatsen. Per annum. (F r. Syst. III. p. 403; R a be $n$ h. p. 100; Botrytis tenera $\mathbf{D}$ z. et $\mathrm{II}$ b. Bijdr. I. p. 29).

Iu Ilerb.: Leiden, Dz. et Mb. (v. s.)

3359. . ochro-rubrum Desinaz. $O_{p}$ rottend hout. Per annum.

Hyphasmate obsoleto; floceis sporidiferis agrogratis ochro-rubris; ramis patentibus, ternis, superioribus oppositis, brevibus; sporidiis minutissimis, glohosis, concoloribus. (Desmaz. Ann. des Sc. Nat. 1834. T. II. p. 71. T. II. f. 5, 5a.)

Ilolland. Splitg. (v. ic.)

\section{ACRTSTACATUS Corda.}

3330. A. cinnabarinus Corda. Op rottende stengels, bedorven aardappelen, rottend hout, enz, op vochtige 
plaatsen. Hieme. (Corda Ic. fung. II. p. 15. T. X. f. 66 ; Rabenh. p. 99 ; Dz. et II b. Bijdr. III. p. 11). In Herb.: Leiden, $\mathbf{D} z_{\text {. }}$ et $M$ b. ( $\mathbf{v}_{\text {. }}$ v. et. s.) $^{\circ}$

BOTHETIS (Mich.) Link.

3391. IB. cinerea Pers, $O_{p}$ oude puisten van Sclerotium durum; alsook op slecht gedroogde kruidgewassen op vochtige plaatsen. Aest.-Vere. (F r. Syst. III. p. 396; Rabenh. p. 96 ; Dz. et MI b. Bijdr. I. p. 29)

In Herb.: Leiden, D z. et $\mathrm{Ml}$ b. (v. s.). Bovendien: Goes, v. d. B.

3392. ㅁ. vulgaris $\mathrm{Fr}$. $O_{p}$ rottende appelen, alsook op rottende vrachten van Cucurbita pepo. Hieme-Vere. (Fr. Syst. III. p. 398; Rabenh. p. 97; Polyactis vulgaris Link; Dz. et II b. Bïdr. III. p. 11).

In Herb.: Leiden, Dz. et $\mathrm{M}$ b. (v. v. et s.)

3393. gels en vruchten. Per annum. (Fr. Syst. III. p. 397 ; Rabenh. p. 97 ; Polyactis cana Dz. et Mlb. Bijdr. III. p. 11).

Mlaastricht, Franq. (n. v.)

3394. IB. parasitica Pers. $0 p$ levende stengels en bladen van Odontites vulgaris, Schoberia maritima en vooral van Capsella bursa-pastoris; parasitisch op de puisten van Cystopus candidus. Aut. (F r. Syst. IlI. p. 403; Rabenh. p. 98; Dz. et II b. Bijdr. I. p. 29)

In Herb.: Hulst, West.; (v. v. et s.); Kattendijke, Zuid-Beveland, v. d. B. Bovendien: Maastricht, Fra n q.

3395. Farinosa $\mathrm{Fr}$. $0 p$ treurende bladen van Rubia tinctorum. Aut. (Fr. Syst. III. p. 404; D z. et II b. Bijdr. I. p. 29)

In IIerb.: Zuid-Beveland, v. d. B. (४. s.) 
3396. violacea liév. Op bloemen van Anthemis cotula. Aut.

Pedicellis erectis continuis dichotome ramosis, sporis ovatis glabris violaceis. (Lév. Ch. du llus. de Par. in Ann. des Sc. nat. $2^{e}$ Série T. V. p. 298; West. Herb. Cr. B. $\mathrm{n}^{\circ} .1199$; Botrytis tenera $\mathrm{D}$ z. et $\mathrm{Il} \mathrm{b}$. Bijdr. 1. p. 22. p. p.)

In Herb.: Zwake (Zuid-Beveland) v. d. B. (v. s.)

\section{NEIIATGONTUMI $\mathrm{D}$ e $\mathrm{s} \mathrm{m}$ a .}

Flocei erecti aut decumbentes, simplices vel subra. mosi, septati et articulali; articulis remotis inflatis. Sporidia vage inspersa, nuda (absque appendiculo), simplicia (non septata), thallo non adglutinata. (Des* maz. Ann. des Sc. nat. 1834. II. 2. p. 69)

3397. N. simplex Desmaz. Op de lamellen van een Agaricus. Aut, (Bonord. Handb. der Hycol. p. 117. T. IX. f. 192)

Leiden, $\mathbf{D}$ z, et $\mathbf{M} \mathbf{b},(\mathrm{v}$, icon.)

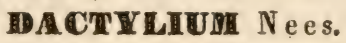

3395. D. dendroides F r. $O p$ een Agaricus trivialis. Aest. (Fr. Syst. III. p. 414; Rabenh. p. 96 ; I)z. et Mb. Bijdr. II. p. 20).

In Herb.: Leiden, Dz. et II b. ( $\mathbf{v} . \mathrm{s}_{\text {。 }}$ )

ASPERGILUS Mich.

3399. A. dubius Corda. $O_{p}$ korsten van oude kaas op vochtige plaatsen. Per annum. (Corda Ic. fung. II. p. 18. T. II. f. 77; R a be n h. p. 94; D z. et Ml b. Bijdr. II. p. 20). In Herb.: Leiden, Dz. en $\mathrm{Ml}$ b.; Zuid-Beveland, v. d. B. (v. s.)

3406. A. glaveus $L i n k .0 p$ slecht gedroogde kruidgewassen. Per annum. (Fr. Syst. III. p. 385; R ahenh. p. 9.3 ; Dz. et M b. Bịdr. I. p. 29) 
In Ilerb.: Leiden, Dz, et Ml.,; Zuid-Beveland? (Goes), v. d. B. (v. 8.)

3401. A. candidus Link. $O_{p}$, doode stengels van groote kruidgewassen. Aut. Hieme. (F r. Syst. III. p. 385; Rabenh. p. $94 ;$ Dz. et MI b. Bijdr. III. p. 10)

Maastricht, Franq. (n. v.)

\section{COHE EMUE $\mathrm{L}$ in $\mathrm{k}$,}

3102. C. vulgare Corda. Op rottende appelen. Per annum. (Corda Prachifl. T. XXV; R aben h. p. 93 ; Coremium glaucum et candidum $\mathbf{D} z$. et $\mathrm{MI}$ b. Bijdr. III. p. 10).

In Herb.: Leiden, Dz. et M b. (v. s.)

\section{TIANUS Corda.}

3403. St. monilloides $\mathrm{C}$ orda. In rottende holle Wilgentronken. Vere. Aut. (R a b e n h. p. 92 ; D z. et Ml b. Bijdr. III. p. 11; Isaria monilioïdes F r. Syst. III. p. 276) In Herb.: Leiden, $\mathbf{D} z$, et $\mathrm{Ml}$ b. (vo s.)

\section{TENICHLLIUTL L ink:}

3404. IP. glaucum $\mathrm{L}$ in $\mathrm{k}, O_{\mathrm{p}}$ bedorven spijzen, alsook up slecht gedroogde planten. Vere. Autumno. (R aben h: p. 91. Penicillium crustaceum Fr. Syst. 11I. p. 407; D z. et $\mathrm{Ml}$ b. Bïjdr. I. p. 29)

In Herb.: Leiden, Dz, et M b. (v. s.)

3405. P. candidum Link. $O_{p}$ een rottenden Agaricus velutipes. Per annum. (Fr. Syst. III. p. 409; Rabenh. p. 91; Dz. et II b. Bijdr. III. p. 10).

In Ilerb.: Leiden, W $t \mathrm{t} w .(\mathrm{v} . \mathrm{s}$ )

3406. De bicolor Fr. $O_{p}$ rollende vruchten, zoo als appelen, peren, enz. - Aut. (Fr. Syst. III. p. 408)

In Herb.: Leiden, D z. et II b. (v, s.) 


\section{IPERONOSTOIEA Corda.}

310\%. IP. densa Rabenh. Op treurende bladen van Rhinanthus crista-galli en Odontites vulgaris.

Caespitibus densissime lanatis, effusis, albidis; stipite ramoso hyalino intricato; ramis dichotomis, ramulis abbreviatis, furcatis; sporis sphaericis, seu ovoideis, seu quadratis, utrinque rotundatis, hyalinis, glabris. (Rabenh. Herb. viv, myc. Edit. $1^{\mathrm{a}} \mathrm{n}^{\circ}$. 1572; l)esmaz. 24e Not. Cr. de Fr. p. 4, no. 7)

In Herb.: Zuid-Beveland (Goes), v. d. B. (v. v. et s.)

3408. P. effusa Desmaz. Op kwijnende bladen van Amarantus blitum, Chenopodium album, Atriplex latifolia, enz. Aut.

Thallo effuso, griseo purpureo pallido. Floceis byalinis densissime intricatis, ramoso dichotomis apice furcatis acutissimis. Sporulis magnis, oviformibus, griseo fulvis. (Desmaz. Pl. Crypt. de Fr. Nouv. Edit. nº 258; Botrytis effusa Grev.; Desmaz. Ann. des Sc. nat. Sér. II. T. VIII. p. 4. T. 1 f. $1 ; \mathrm{D}$ z, et $\mathrm{Mb}$. Bijdr. I. p. 29 p. p.)

In Herb.: Leiden, D z. et $\mathbf{I l}$ b.; v. d. T r.; Blauwkapel, v. d. S. Lac.; Zuid-Beveland, v. d. B. (v. v. et s.)

3109. IP. ganglioniformis Berk. $0 \mathrm{p} \mathrm{kwijnende} \mathrm{bladeu}$

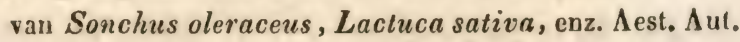

Caespitibus tenuissimis, effusis, albis. Floceis fertilibus parce dichotomis, apice $3-5$ fidis stellatis. Sporulis majusculis, subovoideis, albis. (Botrytis stellata Desmaz, 13 Not. Cr, de Fr. p. 65. no. 4; Peronospora ganglioniformis A. Braun in Rabent. lierb. viv. myc. Edit. nov. $\mathrm{n}^{0}$. 168; Butrylis farinosa 1) z. et Hib. Bijdr. 1. p. 29)

In IIerb.: Zuid-Beveland, v, d, B. (v. vo et s.) 


\section{ACHETHAMNIU⿴囗十⺝ N e es.}

3410. violaceum $\mathrm{Nees}, \mathrm{Op}$ hoomwortels tusschen Ilypnum praelongum. Per annum. (R a ben h. p. 88) Maastricht, Franq. (n. v.)

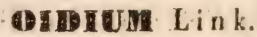

3411. D. aureum Link. Op rollend hout. Aut. Vere. (Fr. Syst. III. p. 429; Dz. et II b. Bijdr. II. p. 20; Torula aurea Corda; Rabenh. p. 37)

In Ilerb.: Zuid-Beveland, v. d. B.; Leiden, Dz. et IIb. ; Harderwijk, Jungh. (v. s.)

3412. fusisporiofdes $\mathrm{F} r$. $0 p$ bladen van verschillende kruidgewassen. Aut. (Fr. Syst. III. p. 431; R a beni. p. 88). Variat:

a. Lapsanae Desmaz. Op bladen van Lapsana communis. (Desmaz. Pl. Cr. de Fr. no. 2134)

In Herb.: Leiden, Dz. et M b; ; Zaid-Beveland, v. d. B. (v. v. et s.)

3413. D. monilioides $\mathrm{L}$ in $\mathrm{k}$. $O_{p}$ bladen van verschillende grassoorten, en bijzonder van Triticum repens. Aut. (Fr. Syst. III. p. 431 ; Rabenh. p. 88; Dz. et M b. Bijdr. I, p. 29)

In Herb.: Leiden, D z. et M b.; Zuid-Beveland (Goes); v. d. B. (v, v. et s.)

3414, 10 . crysiphoides $\mathrm{Fr}, 0_{\mathrm{p}}$, bladen van verschillende kruidgewassen, Aut. (Fr. Syst, Ill. p. 432). Varial:

a. Cucurbitacearum Rabenh. Op bladen van Cucumis sativa (Rabenh. Fung. Eur. exsicc. $n^{\circ}$. 87)

In Herb.: Steeg bij Nijmegren, A bel. (v. v. et s.)

3415. D. fructigenum $\mathrm{K} z \mathrm{e}, 0_{\mathrm{p}}$ rollende vruchten, vonial op pruimen en kersen. Aut. Vere. (Fr. Syst. III. p. $430 ; 0$ c. et II b. Bijdr. II. p. 20; Torula frucligena pers.; Rabenh. p. 37) 
In Herb.: Leiden, Dz. et Il b.; Zuid-Beveland, v. d. B. (v. v. et s.). - Buvendien: Ilaastricht, Fran q.

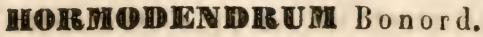

Baumförmig verästelte Hyphen tragen an den Enden der Zweige lange Ketten runder oder ovaler Sporen. (B on or d. Handb. der Mycol. p. 76)

3416. Hi. farinaceum $R$ abenh. $O p$ bladen van $S y m$ phylum officinale. Aut.

Sporis ovatis simplicibus haud septatis, ramis primariis rudimentariis, secundariis articulatis, articulis oblonfyis bipunctatis. (R a benh. Fung. Eur. exsicc. no n $^{0}$ 173)

In Herb.: Kampen, R. B ond. (v. v. et s.)

TORELA Pers.

3418. T. graminicola Corda, Op levende halmen van Holcus lanatus. Aut. (Corda in Sturm. Myc. Ilefte 11I. T. 42 ; Rabenh. p. 35)

Diepenveen, Cop in Herb. Dav. (n. v.)

3418. T. pinophila $\mathrm{Ch}$ e v. $\mathbf{O}_{p}$ takken en bladen van $\boldsymbol{P}_{i}$ nus picea. Aest. Aut. (Fr. Syst. III. p. 231; Rabenh. p. 35)

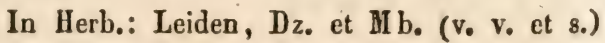

3119. T. Chartarum Corda. Op graauw papier van vochtige plaatsen. Aut. Vere. (Corda Ic. fung. IV. p. 24. T. VI. f. 78; Rabenh. p. 35)

In Herb.: Leiden, $\mathbf{D}$ z. et $M$ b. (v. v. et s.)

34:20. T. herbarum $L$ in $k$. 0 p rottende stengels van kruidgewassen. Hieme. Vere. (F r. Syst. III. p. 501; Ra b e n h. p. 35)

In Ilerb.: Leiden, W tt w.; Amsterdam, v. d. S. Lac. (v, v, el s.) 
3421. T. rhimophila Corda. Op de opgegraven wortels van verschilfende grassoorten. Hieme-Vere. (Cord. Ic. fung. I. p. 8. T. II. f. 127; R a benh. p. 36). Variat:

a. arundinacea West. Op rottende wortels van Phrag-

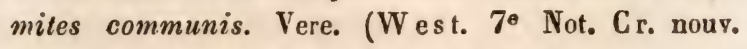
fl. B. $n^{0} .73 ;$ Herb. C r. Belg. $n^{0}$. 1197)

In Herb.: Leiderdorp, $\mathbf{D}$ z. et $\mathrm{M}$ b. (v. v. et s.)

\section{IFISPORA Corda.}

34.22. H. monilioides Corda. Op oude afgehakte boomstammen, palen en houten schultingen. Hieme-Vere. (Rabenh. p. 39; Torula antennata Pers., Fr. Syst. Myc. III. p. $501 ; \mathrm{Dz}$. et $\mathrm{Ml}$ b. Bijdr. I. p. 31)

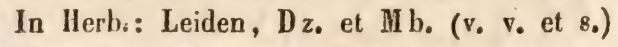

Bovendien: Maastricht, Franq.

\section{Trib. פporotrichei.}

\section{TIR ICHOTHECIUVI Link.}

3423. T. Togenm Link. Op de schors van afyestorven boomtronken, takken, enz., op vochtige plaatsen. Hieme. Vere. (R a benh. p. 86; Fr. Syst. III. p. 427; Dz. et $\mathrm{M}$ b. Bijdr. I. p. 29)

In Herb.: Leiden, Dz. et II b.; Zuid-Beveland, v. d. B. (v. v. et s.)

\section{FUSISFDTRUM Link.}

31218. F. aurantiacum Link. Op rottende IJpenschors, alsook op rottende vruchten van Cucurbita pepo. Hieme-Vere. (F r. Syst. III.p. 445; R a benh. p. 83)

In Herb.: Amsterdam, v. d. S. La c.

3825. F. Thizophilum $W$ est. $O_{p}$ rottende aardappelen en Dahlia-knollen, alsuok op beetwortels in kuilen en kelders. Ilieme-Vere. 
Acervis tuberculifurmibus, convexis, incarnatis, tremellinis, demun effusis, floccis candidis, ramosis, tenerrimis, evanescentibus; sporidiis copiosis, fusiformilus, rectis vel curvatis, hyalinis, $\mathbf{3} \mathbf{- 5}$ septatis. (Desmaz. Mém. Soc. Roy. des Sc. de Lille. Vol. 1827-28. p. 453.). Variat:

a. Dahliae West. Op de rottende knollen van Dahlia. (W es t. 2e Not. Cr. inéd. fl. B. p. 36. no 194 )

In Herb. Leiden, $\mathbf{D}$. et $\mathrm{M}$ b. (v. v. et s.)

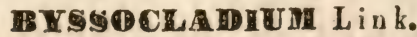

3426. IB. fenestrale $L$ ink. $O_{p}$ vensterglazen in vochtige vertrekken, zoo als kelders, stallingen, enz. Aut. Verc. (Rabenh. p. 82)

In Herb.: Hulst, West. (v. v. et s.)

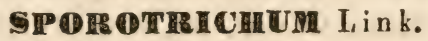

3428. F. candidum Link. Aan cene schutting on op cenc verrotte blaas in een kelder. Hieme. (Rabenh. p. 78; Dz. et II b. Bijdr. III. p. 11)

In Herb.: Leiderdorp, de II aan. (v. s.)

3428. \$. laetum Link. $O p$ doode takken in vochtige kelders. Hieme. (F r. Syst. III. p. 423 ; R a be $\mathbf{n h}$. pag. 80)

In Herb.: Amsterdam, v. d. S. L ac. (v. vo et s.)

3429. 5. flavicans $\mathrm{Fr}$. $\mathrm{O}_{\mathrm{p}}$ doode takken en hout van Pijnboomen. Aut. Vere. (Fr. Syst. III. p. 119; Dz. et II b. Bïdr. 1. p. 29)

In Herb.: Leiden, Dz. et Mil b.; Harderwijk, Jungh.

3430. G. aureum Link. (non Pers.) Op rotlend hout en schors van Elzen. Hieme. (Rabenh. p. 80; Sporotrichum aurantiacum Fr. Syst. III. p. 423)

Naastricht, Franq. (n. v.) 
3431. 5. fusco-album Link. $O_{p}$ rottend hout. Hiemc. (R a benh. Deutschl. II. I. p. 81; Dz. et Mb. Bijdr. III. p. 11)

In Herb.: Zuid-Beveland (Goes), v. d. B. (v. 8.)

3432. \$. olivaceum Fr. Op rottend hout en rottenden bast. Ilieme. (R abenh. p. 82)
In Ilerb.: Ansterdam, v. d. S. Lac. (v. 8.)

3433. S. cinereo-virens $\mathrm{Fr}$. $\mathrm{Op}$ rottende stengels van Umbelliferae. Hieme. (Fr. Syst. III. p. $416 ; \mathrm{D} z$. et II b. Bïdr. I. p. 29)

In Herb.: Leiden, Dz. et II b. (v. s.)

3434. 5. bryophilum Pers. $O_{p}$ bemoste Wilgentronken. Aut.

Laxum crassiusculum, floccis intricatis, sporulis conglomeratis globosis demum fuscescentibus. (Pers. Myc. Eur. I. p. 78; Dz. et II b. Bijdr. IH. p. 11)

In Herb.: Leiden, Dz. et $\mathrm{Ml}$ b. (v. s )

\section{GEOTRICHUM $\mathrm{L}$ in $\mathrm{k}$.}

3435. Cr. candidum Link. Op vochtige turfaarde in de bosschen. Per annum. (Rabenh. p. 77)

Maastricht, Franq. (n. v.)

\section{Trib. Sepedonei.}

\section{C聠LORIDIU⿴囗十⺝ L ink.}

3436. Ch. festucae Corda. Op bladen van grassen in vochlige bosschen. Aut. (Corda. Ic. fung. III. p. 7. Tab.1. f. 19 ; Rabenh. p. 75) Amsterdam, O udem. (v. i.)

SEPEDONHURI Link.

.3438. 5. mycophilum Link, $0_{p}$ stervende en rottende 
Fungi, an bijzonder op Bolelus-soorteu. Aut. (R abeuh. p. 71; Sepedoniam chrysospermum Fr. Syst. III. pag. 438 ; Dz. et 11 b. Bijdr. I. p. 29)

In Herb.: Leiden, Dz. et II b.; Zuid-Beveland, v. d. B. (v. v. et 8.). Bovendien: Maastricht, Franq.

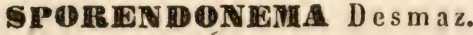

3438. Sp. casei Desmaz. $O_{p}$ korsten van oude kaas. Aut. Vere. (Fr. Syst. III. p. 435; D z. et Il b. Bijdr. 111. p. 11; Sepedonium caseosum, Link; Rabenh. P. 7I)

In Herb.: Utrecht, Dz. et Ml b. (v. vo et s.)

\section{RSILONIA Fr.}

3439. P. gilva $\mathrm{Fr}, \mathrm{O}_{\mathrm{p}}$ doode Brandnetelstengels. Hience Vere. (Fr. Syst. 111. p. 451, Kabenh. p. 73; Cono. plea gilva Pers.)

In Herb.: Leiden, Dz, et II b. (v. v. et so)

\section{COLLAREM Link.}

3410. C. nigrospermum Link. Op rottende of verdroogde lijm. Aut. Vere. (Rabenh. p. 70; Fr. Syst. III. p. 441 ; Sporotrichum collac Dz. et II b. Bijdr. 11I. p. 11)

In Herb.: Leiden, $\mathbf{D}$ z. et $\mathrm{Ml}$ b. (v. s.)

*\# \# ysacei.

Trib. Phylleriacel.

TAPITINA Fr.

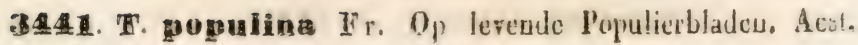


Aut. (Fr. Syst. 111. p. 520; R a be n l. p. $6 \dot{4}$; Erineuna aureum Pers.; Dz. et M h. Bijdr. 1. p. 39)

In Herb.: Leiden, Dz. et $M$ b.; te Beeklust in de Ooi en te Heumen, A bel.; Zuid-Beveland, vo d. B. (v. v. et s.)

Bovendien: Maastricht, Franq.

\section{CIRENUII Pers.}

3412. Woseum Schulı. $O_{p}$ levende bladen van $B c$ tula alba. Aut. (Rabenh. p. 65; Dz. et Mb. Bijdr. I. p. 38)

In llerb.: Beek, A bel.; Amsterdam, M. Doorn. (v. v. et s.)

3143. He. betulinum Pers. Op levende hladen van $B c$ tula alba. Aul. (Rabenh. p. 65 ; Fr. Obs. I. p. 221)

In llerb.: Bloemendaal, 0 u de m.; Leiden, D z. et II b. (v. vo et s.)

3444. Platanoideum Fr. $O_{p}$, bladen van Acer platanoides. Aut. (Fr. Obs. 1. P. 224; Rabenh. p. 65 ; 1) z. et H b. Bijdr. 1. p. 38)

In Ilerbo: Leiden, Dz。 et HI b. (vo vo et s.)

Bovendien: Maastricht, Franq.

3445. Dopulinum Pers. $O_{p}$ levende Populierbladen, $\Lambda$ ut. (Fr. Obs. 1. p. 223; Kabenh. p. 65; Dz. et II b. Bïjr. 1. p. 38j

In Herb.: Zuid-Beveland, v. d. B. (v. v. et s.)

Bovendien: Maastricht, Franq.

3146. E. alneum Pers. Op levende bladen van Alnus glutinosa. Aul. (F r. Obs. I. p. 223; Rabenh. pag. $65 ; \mathrm{D}$ z. et $\mathrm{Ml}$ b. Bïjdr. I. p. 38)

In Herb: Leiden, Dz. et $11 \mathrm{~b}$.; Haarlem, Splitg; Kampen, R. Bond; Nijmegen, A bel. (v. v. et s.)

buveudieu: Maastricht, Franq. 
3448. Eagineum Pers. Op Beukenbladen. Aut. (Pers. Obs. II. p. 102; Rabenh. p. 66; Dz. et $\mathrm{Ml}$ b. Bijdr. I. p. 38 )

In Herb.: Haarlem, Splitg. (v. vo et s.) Variat:

a pallidum Link. (F ée. Mém. sur les Phyll, p. 55)

In Herb.: Leiden, Dz. et II b. (v. v. et s.)

b. purpureum Dec. (Fée. I. I. p. 54; Dz. et $\mathrm{Hb}$ b. Bijdr. I. p. 38)

In Herb.: Leiden, $\mathrm{Dz}$. et $\mathrm{Mb}$. (v. v. et s.)

34418. D. padi Duv. et Roeh!. Op bladen van Prunus padus. Aut. (Rabenh. I. p. $66 ; \mathrm{D} z$. et $\mathrm{H}$ b. Bijdr.

I. p. 39.)

In Herb.: 't Ilaaggche Bosch, Dz. et II b. (v. v. et s.)

3419. E. oxyacandhae Pers. Op bladen van Crataegus oxyacantha. Aut. (R a benh. p. 66; Er. clandestinum, Grev.; Dz. et $\mathrm{Ml}$ b. Bijdr. I. p. 39).

In Ilerb.: Leiden, Dz. et Il bo; Zuid-Beveland, v. d. B. (v. v. et s.)

Bovendien: Mlastricht, Fran q.

3450. E. purpurascens Gaertn. et Roehl. Op bladen van Acer campestre en platanoides. Aut. (R abenh. p. 66; L. acerinum Schum.; Dz. et IIb. Bijdr. I p. 38).

In Herb.: Leiden, $\mathbf{D}$ z. et $\mathrm{Ml}$ b.; Beek bij Nijmegen, A bel. (v. v. et s.)

Bovendien: Maastricht, Franq.

\section{PHYLLEISERT Fries.}

3151. Ph. tiliaceum Pers. $0 p$ levende Lindebladen. Aut. (P e r s. Syst. p. 700 ; Cord. Ic. fung. V. p. 48. T. 1. f. 1; Erineum tiliaceum Pers, D z et $\mathrm{Mb}$. Bijdr. I. p. 38), 
In Herb: Ilaarlem, $\mathrm{S}_{p}$ lit gr ; Leiden; $\mathrm{D}_{z}$. et il b.; Utrecht, v. d. S. L a c.; Ubbergen, A bel.

Bovendien, Maastricht, F r a n q.

3452. Ph. juglandis Schleich. in Dec. Op levende bladen van Juglans regia. Aut. (R abenh. p. 67; Er. joglandinum Fries; Dz. et Ill b. Bijdr. I. p. 38).

In Herb.. Leiden, Dz. et $\mathrm{Mb}$.; Haarlem, S plitg.; Weurt bij Nijmegen, A b e l.; Amsterdam, v. d. S. L a c. ; Kampen, R. Bond,; Groningen, Strat. (v. v. et s.) Bovendien: Maastricht, Franq.

3453. Ph. axillare $F$ ée. $0 p$ bladen van Alnus glutinosa. Aut. (Fée Mérn. sur ies Phyll, p. 33)

In Herb.: Rotterdam, $\mathrm{O}$ udem. (v. s.)

3454. Ph. pyrinum Fr. Op bladen van Pyrus malus. Aut. (Rabenh. p. 67; Erineum pyrinum Per8. Syn. p. 700 ; Dz. et. $\mathrm{H}$ b. Bïjdr. I. p. 38).

In Herb.: Leiden, Dz. et $M b$. (v. v. et s.)

Bovendien : Maastricht, Franq.

3455. Ph. rubi Fr. $O_{p}$ bladen van verschillende Rubussoorten. Aut. (Fée. Mérn. sur les Phyll. p. 43; Erineum rubi Pers.; West. Herb. Cr. B. $\mathrm{n}^{0} .590$; D z. et $M$ b. Bịjdr. II. p. 22).

In Herb.: Nlaastricht, Franq. (v, v. et s.)

3456. Ph. bifrong Lepell. $O p$ levende Lindebladen. Aut. (Fée Mém. sur les Phyll. p. 41)

In Herb.: Leiden, O udem. (v. s.)

345\%. Ph. pseudoplatani $\mathrm{Kz}$. $0 \mathrm{p}$ bladen van Acer pseudoplatanus. Aut. (Raben h. p. 67; Er. pseudoplatani Kze; Dz. et M b. Bijdr. I. p. 38).

In Herb.: Leiden, Dz. et Ml b.; Kampen, R. Bond.; Ubbergen, A bel. (v. v. et s.)

3458. vitis Fr. Op hladen van Vitis vinifera. Aut. (Rabenh. p. 67; Erineum vitis Dec; Diz. et Mb. Bịjls. I. p. 38). 
In Herb.: Leiden, Dz. et $\mathbf{I l}$ b.; Zuid-Beveland, จ. d. B.; Haarlem, Splitg.; Dordrecht, Utrecht, v. d. S. L a c.; Nijmegen, A b e l.; Amsterdam, II. Doorn.; Kampen, R. Bond. (v. v. et s.)

Bovendien: Maastricht, Franq.

3459. Ph. alnigenum $R$ abenh. Op bladen van Alnus glutinosa. Aut. (Rabenh. p. 67; Erineum alnigenum $\mathrm{K} z \mathrm{e})$

Maastricht, Franq.

\section{Trib. Destructorel.}

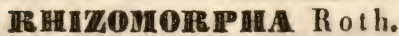

3460. IB. subcorticalis Pers. Tusschen het hout en den bast van stervende boomtronken, alsook op run in broeibakken. Per annum. (R a benh. p. 63 ; D z. et II b. Bijdr. I. p. 28)

In Herb.: Leiden, Dz. et Mb.; Rijnsburg, R. Bond.; Amsterdam, v. d. S. Lac.; Paterwolde, Zwijndrecht, v. d. S. Lac.; Werkendam, v. d. S. Lac.

Bovendien: Zuid-Beveland, v. d. B.; Maastricht, Franq. (v. v. et s.) Variat:

a. latissima $\mathrm{Kx}$. Rech. fl. crypt. Fland. 5 Cent p. 24) In Herb.: Utrecht, v. d. S. Lac.

b. tendo Pers. Tusschen het hout en de schors van Pijnboomen. (Pers. Myc. Eur.; Rh. subcorticalis flaccida Chev. fl. Par. I. p. 507)

In Herb.: Harderwijk, Jungh. (v. s.)

3461. Mh. divergens Grev. Tusschen het hout en de schors van Dennen- en Hastboomen. Per annum. (Rabenh. p. 64; Dz. et Mb. Bijdr. I. p. 28)

In Herb.: Harderwijk, Jungb. (v. s.)

3162. Ith. parallela Rob. in Desmaz. Op en in doode Vliertakken. Aut. Hieme. (Rh. sambuci Chev.) 
Nigyra laevigata, caulihus filiformibus longrissimis aequar. libus parallelis nee intricatis nec ramosis. (Chev. 11. Par. I. p. 508)

In Herb.: Amsterdam, v. d. S. Lac. (v. v. et s.)

3163. Hh. setiformis Roth. $O_{p}$ Sphagnum en andere mossen in vochtige bosschen. Per annum. (Roth. Cat. 1. p. 235; West. Herb. Crypt. B. nº.548. Ceratonema hippotrichodes Pers.)

In Herb.: Breda, vo d. S. Lac.; Nieuw Loosdrecht, v. d. S. Lace (v. vo et s.)

3161. Ith. intestinails Pers. Tusschen de vezels van rottend Olmenhout. Hieme. (D z. et II b. Bijdr. I. p. 28) In Herb.: Leiden, Dz. et II b. (v. s.)

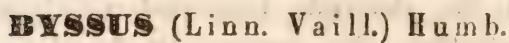

3165, H. floccosa Schreb. Op muren en balken in vochtige kelders, Per annum. (R abenh. p. 62; liypha bombicina Pers.; Dz. et Ml b. Bijdr. II. p. 20) In Herb.: Leiden, $\mathbf{D z}$. et II b.; Zuid-Beveland (Goes) v. d. B. (v. s.)

\section{OLONIU⿴囗十⺝ (P e rs.) Link.}

3166. D.-auricomum Link. $O p$ rottend hout op zeer vochtige plaatsen. Per annum. (R a benh. p. $60 ; 0 z$. fulvuin Pers.; Dz. et II b. Bijdr. I. p. 28)

In Herb.: Amsterdam, v. d. S. La c.; Zuid-Beveland, v. d. B. (v. v. et $s_{0}$ )

316\%. 9. parietinum Link. $0 p$ muren in vochtige kelders. Hieme. (R a benh. p. 61)

In Herb.: Ouwendijk hij 's Gravesande.

3168. 6. castaneum $W$ all r. $O_{p}$ rottend Eikenhout. Hieme. (Rabenh. p. 61) 
In IIerb. Nijmegen, A bel.; Luid-Beveland, v. d. B. (vos.)

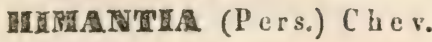

Flocei decumbentes fasciculati intertexti, apice radiantes; sporulac passim golohosae. (Chev. fl. Par. I. pag. 80)

319D. 顷. camelida Pers. $O_{p}$ afgevallen rottende bladen. Hieme. (Pers. Syn. p. 703; Ozoniun candidum IIart.; Rabenh. p. 61)

In Herb.: Leiden, Dz. et Mb.; Zwake, v. d. B. (v. vo et s.) - Bovendien: Maastricht, Franq.; Naaldwijk, v. d. Tr.

3480. II. radians Pers. Op afgevallen bladen in de bosschen. Hieme.

Alba, folia agglutinans, filis in fasciculum confertis apice divergentibus. (P ers. Syn. p. 703)

In Herb.: Leiden, $\mathbf{D} z$. et $\mathbb{M}$ b. (v. s.)

3481. 14. rufipes $C h e v, O p$ rottende vaten in vochtige kelders en oranjeriên. Hieme. Vere.

Filis divergentibus implexis albidis, ad basin contextissimis compactis, subtomentoso-spongiosis rufescentibus. (C h e v. fl. Par. I. p. 81)

In Herb.: Leiden, in den Hortus Academicus. W $t \mathrm{t}$. (v. v. et s.)

\section{Trib. Heteorici.}

\section{X}

342. X. giganteums 'índe. 0p rottend hout. Aut. Vere. (Tode. Tung. IIeckl. sel. I. p. 36. T. VI. f. 51; $\mathrm{X}$, Corium Rabenh。p.60) 
In Ilerbs: Leiden, in 's Rijks plantentuin. de II a an. (v. v. et s.)

\section{ATHWHA Pers.}

Tilamenta intertexta radiantia medio in pelliculam fenucm laevem depressorapplanalam fibrillis sporulis intermistis obductam condensata, radio libera. (Duby, Bot. Gall. II. p. 932.)

31.8. A. Cpiplaylla Pers. Op rottende mossen en bladen op den grond in de bosschen. Aut. Vere. (Pers. Champ. Com。 j. 67; Thelephora epiphylla Fr. Elcnch. I p. 226).

In Ilerb: : Zuid-Beveland (Goes) $\nabla_{0}$ d. B. 


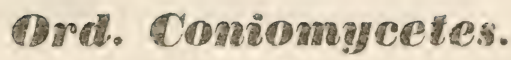

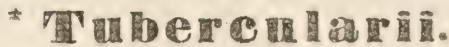

\author{
Trib. Tribercularini.
}

EIPICCEUPR Link.

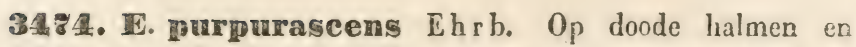
bladen van Zea mais. Aut. Hieme. (Corda Ic. fung. I. p. 5. T. 1. fig. 90; E. versicolor b. purpurascens R a benh. p. 58)

In Herb。: Leiden, in 's Rijks plantentuin. Jungh. in Herb. $d e r_{0}\left(v_{0}, s_{0}\right)$

\section{PETIOHA Fries。}

3185. T. tomentosa $\mathrm{Fr} .0 \mathrm{p}$ roftende aardappelen in vochtige kelders. Hieme. (F r. Syst. II. p. 521; Rabenh. p. 56)

In Herb.: Dongen in Noord-Braband (v. v. et s.)

\section{CHIA}

39. Ch. Buxi Corda. Op doode bladen van Buxus sempervirens. Verc. Aut. (Corda Ic. fung, II. p. 30. T. XIII. f. 107; Fusisporium Buxi Fr. Syst. III. p. 447; Fusidium Buxi Sehm.; R abenh. p. 42) In Herb, Bommel, Jungh。 in Ilerb.W $\mathbf{t}$ w: 


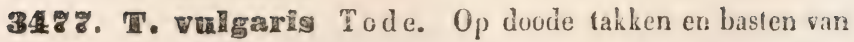
hoomen. Vere. Aut. (Fr. Syst. III. p, $464 ;$ Raben h. p. $53 ; \mathrm{D}$ z. et $\mathbb{M}$ b. Bijdr. I. p. 30)

In Herb.: Heikop, de Ilaan; Leiden, Oudem。, Wt tw.; Willemsdorp, Zuid-Beveland, จ. d. B.; Gro= ningen, de Наan。 (

348. T. Thertoarum Fr. Op roticnùc koolstronken. Ilieme. (Fr. Syst. 111. p. 465; Rabenh. p. 54)

In llerb.: Amsterdam, v. d. S. Lac. (v. s.)

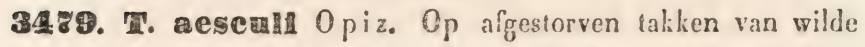
Kastanjes (Aesculus hippocastanum). Vere. Aut. (Corda Ic. fung. I. p. \&. T. 1. f. 77; T. vulgaris o. Aesculi Rabenh. p. 54)

In Herb.: Leiden, $\mathrm{D}_{\text {z }}$ et $\mathbb{M} \mathrm{b}$; Weikenburg bij Utrecht, W $t \mathrm{tw}$. ( $\nabla_{0} \nabla_{0}$ et $\mathrm{s}_{\circ}$ )

3480. T. Conllitems Pers. Op afgehouwen tronken van Acer, Crataegus; enz. Hieme. (Corda 1. I. fig. 74; Rabenh. p. 54; Dz, et MIb. Bijdr. I. p. 30)

In Ilerb.: Leiden, $\mathrm{D} z$. et $\mathrm{M}$ b.; Kampen, R. Bond.; Eekta (Groningen) Strat.; Sas van Gendt, Walr.; Valkenburg bij Leiden, W $₫$ w.; Maastricht, Franq. (v. v. et s.)

348耳. T. metabilis Necs. Cp doode takken van Kerria japonica, in de lusthoven. Aut. Vere. (Corda I. I. fig. 73; Rabenh. p. 55; West. Herb. Crypt. Belg. $\left.\mathrm{n}^{\circ} \cdot 1191\right)$

In Herb.: Amsterdan, v. d. S. La c. (v. vo et s.)

3482. 冝. 进oceosa Link. Op doode schors ran Aluus, Betula, enz. Ilieme. ( $\mathrm{R}$ abenh. p. $55 ; \mathrm{D}$ z, et $\mathrm{Ml}$. Bịdr. III, p. 11; T. floccipes Corda.)

In Herh.: 'Aan de Delftsche vaart, de ll a a $n,(v, \delta)$ 


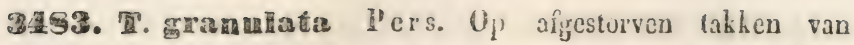
Cornus mascula, Pyrus malus, enz, Hieme. Vere. (Corda 1. 1. f. 75; Rabenh. p. 55)

In Ilerbs: Leiden, W $\mathrm{ttw}_{\mathrm{w}}$ ( (v. s.)

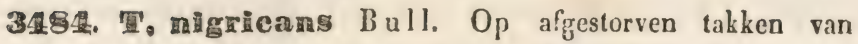
pijn- en mastboomen. Hieme. Vere. (R abenh. p. 55) In Herb.: Zuid-Beveland, v. d. B. (v. vo et s.)

330. T. expallems Fr. ap. Desmaz. Aan cene schutting. Ilieme. (Fr. ap. Desmaz. Pl. Cr. de Fr. $n^{0}$. 171; We st. Herb. Crypt. B. ${ }^{\circ}$. 396)

In IIerb.: Utrecht, v. d. S. L a c. (v. s.)

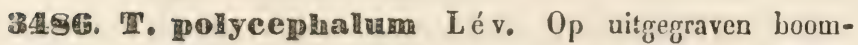
wortels. Ilieme. Vere. (Lév. ap, Dz. et Mi b. in lierb. Un. $\left.n^{\circ} .243\right)$

In IIerbo: Leiden, D zo et II b. (vo s.)

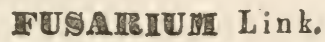

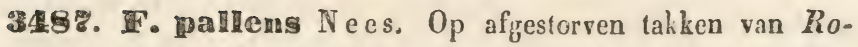
binia Pseudacacia. llieme. (Rabenh. p. 50; Volutella pallens Fr. Syst. III. p. 468)

Maastricht, Franq.

3488. Foseum Link. Op rotiende stengels van Malva sylvestris, alsook op rottende koolstronken. Hieme. (Rabenh. p. 50; Corda Ic. fung. I. p. 3. T. I. f. 55)

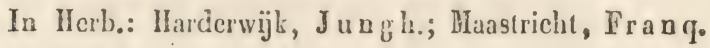
v. v. et s.)

34189. Fi Theterosporium $\mathbb{N}$ ecs. In de zaden van stervende grasplanten, zooals van Lolium perenne, Glyceria fluitans, enz. Aut. (F r. Syst. III. p. 372; Rabenh. p. 51 ; D z. et $\mathbb{M}$ b. Bijdr. I. p. 30)

In Ilent): Leiden, Dz. et Mib.; Maastricht, Franq. ( $\because$. v. et is) 


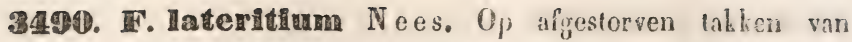
Mlorus alba. Hieme. Vere. (Fr. Syst. III. p. 470 ; Rabenh. p. 51)

In Herb.: Mlaastricht, Fran q. (vo vo et s.)

3401. F. sulphuream $S \operatorname{chl} l d l$. Op rottende aardappelen in vochtige kelders. Hieme. (F r. Syst. III. p. 471; Dz. et II b. Bijdr. II. p. 20; Fusidium sulphureum Link; Rabenh. p. 42)

In Herb.: Leiden, Dz. et $\mathrm{Ml}$ b. (v. s.)

3192. F. pezizoides Desmaz. Op stervende en doode bladen van Hedera helix, dikwïls gemengd met Peziza hederae Lib. Aut. Vere.

Erumpens, amphigenum, subiminersum, minufum, numerosum, sparsum, rotundatum, pallide rufidulum, siccum concarum, humidum convexum disciforme, gelatinosum, opalinum, subbyalinum. Sporophoris monosporulis, simplicibus, pedicelliformibus; sporulis ovoideis, hyalinis. (Desmaz. $20^{\circ}$ Not. Crypt. de Fr. p. 19. no. 21; Desmaz. Pl. Cr. de Fr. Exs.no. 2176)

In Herb.: Leiden, Zuid-Beveland, vo d. B.; Amsterdam, v. d. S. Lac. (v: v, et s.)

3903. F. equisetorum Desmaz, Op stervende stengels van Equisetum limosum. Aut.

IIInutulum, punctiforme, erumpens, sparsum, rotundum, convexum, carnoso-tremellosum, rufo-ochraceum. Basidiis filiformibus, continuis; sporulis acrogenis, minutissimis ovoideis, hyalinis. (Desmaz. $17^{\mathrm{e}}$ Not. Crypt. Nouv. de Fr. p. 37. no. 55; Desmaz. Pl. Cr. de Fr. no. 1846)

In Ilerb.: Dongen in Noord-Braband. (v. v. et s.)

GHOLDG

Perithecium nullum. Sporae ex ovoideo-pyriformes aut subeylindricae, subcontinuae, hyalinae, pedicello (sporophoro) e parenchymate folii exorto suffultae, mos 
libcrac et sub epidermide collectac, tandom, hac rupta, ope mucilaginis subcirrose rejectae, eirris achromaticis。 (II o n t. Syll. Gen. Spec, Crypt. p. 195)

\$1919. CHI. acerinum West. Op kwijnende bladen van Acer campestre. Aut.

Taches hypophylles, pâle-jaunâtres, grandes, irréguulières, attaquant de préférence les bords de la feuille. Réceptacles rouge-brunâtres, ponctiformes, nombreux, éparpillés sur le pourtour de la tache, d'abord reconverts par l'épiderme, puis nus par la déstruction de celle-ci. Sporidies ovale-allongées ou presque cylindriques, hyalines, mesurant $1 / 50^{\mathrm{e}}$ à $1 / 100^{\mathrm{e}}$ de mill. de longueur sur $1 / 400^{\circ}$ de mill. de largeur. (W est. $4^{\circ}$ Not. C r. Nouv. fl. Belge. p. 7. no. 17; W est. I.C.B. n०. 979)

In Herb.: Leiden, Dz. et $\mathbf{I I} b_{\text {: }}$ (v。 vo et s.)

3195. GH. almeum West. Op treurende bladen van Alnus glutinosa. Aut.

Taches hypophylles, rousses, arrondies, à bords anguleux et irréguliers, de 5 à 7 mill. de diamêtre, éparses ou rarement confluentes. Le centre des taches, qui devient d'une teinte brun-grisâtre, est couvert d'un grand nombre de petits points bruns, bulleux, emettant, après la rupture de l'épiderme, des sporidies hyalines, ovales, de $1 / 200^{\circ}$ de mill. de longueur sur $1 / 400^{\circ}$ de mill. de largeur. (West. l. 1. p. 7. no. 18; West. Ilerb. C. B. no. 977)

In Ierb。: Leiden, $\mathrm{Dz}_{\text {。 }}$ et III b. (v. vo et s,)

3496. GH. quereingum Wes: Op kwijnende Eilenbladen. Aut。

Taches hypophylles, inun-verdâtres, éparses ou conlluentes, couvertes d'un grand nomlie de réceptacles fonctiformes, bulleux, rousces ou rougendurunàtres, épara pillés de préférence sur le pourtour de la tache. Après 
la déstruction de l'épiderme, on voit de très petits gulobules orangés, translucides comme de la cire, formés par un mucilage contenant des sporidies ovale-allongées, hyalines, de 4 a $5 / 100^{\text {es }}$ de mill. de longueur $\operatorname{sur}^{1} / 200^{\mathrm{e}}$ de mill, de largeur. (W est. 1.1. p. 8. $\mathrm{n}^{\circ} .21$; Westo Herb. Cr. B. $n^{\circ}$. 981)

In Ilerb.: Leiden, $v$, $d, B$, ( $v$, v, et s.)

319\%. GH. Betaliae We st. Op kwijnende bladen van Betula alba. Aut.

Taches hypophylles, rousses, arrondies, irrégulières, de grandeur variable, couvertes d'un grand nombre d'autres petites taches bulleuses d'un roux plus clair, formées par l'épiderme soulevé. A la maturité celle-ci 8e rompt irrégulièrement pour laisser a nu la masse sporidifère, également rousse, formée d'une grande quantité de sporidies hyalines, ovoïdes, de 2 à $3 / 100^{\text {es }}$ de mill. de longueur sur $1 / 400^{\circ}$ de mill. de largeur. (West. 1. 1. p. 7. $n^{\circ} .20$; West. Herb. Cr. B. n'. 978)

In Herb.: Leiden, $\mathrm{Dz}_{\text {c }}$ et $\mathrm{MI} \mathrm{b}$. (v. v. et s.)

3198. GH. sallicis W est. $O p$ treurende Wilgenbladen. Aut. Taches épiphylles, brunes ou brun-rougeâtres, petites, éparses ou confluentes, arrondies ou irrégulières. Réceptacles plus pâles, porictiformes, nombreux, bnlleux, ćmettant, après la rupture de l'épiderme, des sporidies hyalines, ovales, mesurant à peine $1 / 200^{\mathrm{e}}$ de mill. dans le grand diamètre. (W est. 7 e Not. C r. inéd. fl. B. p. 12. $\mathrm{n}^{\circ}$. 44 ; West. IIerb. Cr. Belg. $\mathrm{n}^{\circ}$. 1269)

In Herb.: Zuid-Beveland, v. d. B. (v. v. et s.)

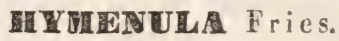

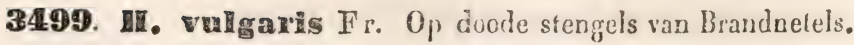
Hieme. Vere. (F r. Syst. II. p. 234; R a benh.p.50) In Ilero. I Irastricht, Fran q-; Zuid-Beveland, v. d. B. $(\mathrm{r}, \mathrm{s})$ 


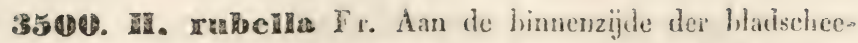
den van Typha latifolia. Ilieme. Vere. (F r. Elench. 1I. p. 38 ; R abenh. p. 50)

In Ilerb.: Zuid-Beveland, v. d. B.; Mlaastricht, Franq. ( v. v. et s.)

B501. II. arum ahinis F r. Op afyevallen halmen van Phragmites vulgaris. Aut. Vere. (Fr. Syst. II. p. 234; F r. Elench. II. p. 37; R abenh. p. 50)

ilaastricht, Franq.

\section{Trib. Stilloosporel.}

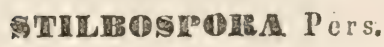

3542. פo macrosperma Pers. Op afgestorven basten en takken van verschillende boomen. Aut. Vere. (Fr. Syst. III. p. 485; Rabenh. p. 47)

In, Ilerb.: Zuid-Beveland, v. d. B. (v. v. et s.)

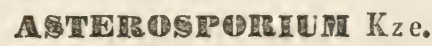

Sporidia coacervata, stellata, septata, stromati heterogeneo fluccoso-granuloso incumbentia. (Kze. Bot. Zeit. 1819. 1. 1. p. 225)

35๑3. A. hofrmanni $\mathrm{K} z \mathrm{e}, \mathrm{O}_{\mathrm{p}}$ doode taken van Berken en Beuken. Aut. Vere. (Kze. I. I.; Stilbospora asterosperma Pers.; Rabenh. p. 47; Fr. Syst. 1II. p. 484) In Herb.: Zuid-Beveland, v. d. B. (v. v. et s.)

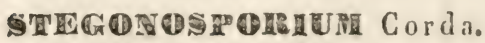

Perithecium immersum, membranaceo-flocesum, tenuissimum, dein supra irregulariter apertum. Stroma columellaeforme vel nullum. Pulpa sporarum atra, dein crumpens, Basidia filiformia, paraphysibus mixla. 
Sporæ acrogenae; episporio contimus unilueulari; nucles transverse septato vel celluloso. (C orda Ic. fung. III. p. 22)

3501. St. pyriforme Corda. Op afgestorven takken on schors van Acer campestre. Aut. Vere.

Gregarium. Peritheciis immersis, semper tectis, coriaceis atris; basidiis filiformibus albis; sporis obovatis vel pyriformibus; episporio duplici, pallido; nucleo celluloso-fusco; cellulis plerumque transverse stratosis. (Corda. I. I. III. p. 23. T. IV. f. 61; West. Herb.: Cr. Belge $n^{\circ} .577$ )

In Herb.: Zuid-Beveland, v. d. B. (v. v. et s.)

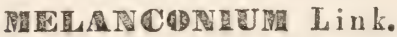

3505. HI. sphacrospermum Link. Op doode en afgevallen halmen van Pliragmites communis. Hieme. Vere. (Fr. Syst. III. p. 489; Raben h. 1) 44; Dz. et M b. Bijdr. I, p, 30).

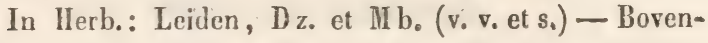
dien: Naastricht Franq.; Naaldwijk, v. d. Tr.

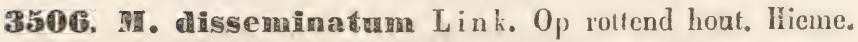
(Fr. Syst. III. p. 489 ; Rabenh. p. 44; Dz. et Hi b. Bijdr. I. p. 30)

Leiden, $\mathbf{D} z$. et $\mathbb{I l} \mathrm{b}$.

350\%. MI. ovatum Link. Op schors van omgehakte boomen. Hieme. Vere. (Rabenh. p. 44; Stilbospora ovata Pers.; Fr. Syst. III. p. 485; Dz, et Mb. Bijdr. 1. p. 30)

In llerb.: Langs de Delfisehe vaart, de $\mathrm{H}$ a a $\mathrm{n}$ in Hlerb. De V r. - Bovendien: Maastricht, Franq.

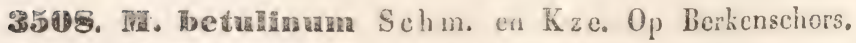
Hieme. (R $a b \in n$ b. p. 45)

In Herb: Hastricht, Franc. 


\section{IBLENNDER $\mathrm{F}$.}

3509. Waxi Fr. Op doode hladen van Buxus sempervirens. Aut. (Fr. Syst. III. p. 472; Rabenh. p. 43) In Herb.: Bommel, W $t \mathrm{t}$ w. ex herb. Jungh (v. v. et s).

\section{FUG}

3510. T. candildum $\mathrm{Link}, 0 \mathrm{p}$ afgestorven Beukenschors. Ilieme. Vere. (R aben h, p. 42)

In Herb.: Dongen in Noord-Braband. (v. s.)

35耳. F. hysteriaeforme West. $O_{p}$ overrijpe meloenen en pompoenen. Aut. Vere.

Pustules irrégulières, allongées, éparses ou confluentes, blanchâtres, luisantes, bulleuses ou boursouflées; l'épiderme du fruit qui. les recouvre se fend longitudinalement, s'écarte et montre des rainures remplies d'une poussière farineuse, très blanche, formée de sporules nombreuses, hyalines, ovales, ovale-allongées ou presque cylindriques, parfois offrant une cloison au milieu et mesurant $1 / 200^{\mathrm{e}}$ à $1 / 50^{\mathrm{e}}$ de mill. de longueur sur $1 / 300^{\mathrm{e}}$ à $\mathbf{1}_{200^{\mathrm{e}}}$ de mill. de largeur. (W est. 4e Not. Cr. inéd. 11. B. p. 14. no.70; West. II.C.B. $\left.\mathrm{n}^{\circ} \cdot 1290\right)$

In Herb.: Zuid-Beveland (Goes), v. d. B. (v. v. et s.)

3512. F. parasidicum W est. Op het stroma van Xylaric cornuta, dat hij doet treuren en verdroogen. Hieme.

Couche d'abord gélatineuse, puis se concrétant en une poudre farineuse, très blanche, formée par des sporules excessivement petites, nombreuses, cylindriques, fusiformes, transparentes, n'ayant qu'environ ${ }^{1} / 50^{\circ}$ de mill. de longueur sur une largeur moyenne de ${ }^{1 / 200^{e}}$ de mill. (West. 3 e Not. Crypt. , inéd. fl. B. p. 35. no. 189; WV est. H.C.B, no $n^{\circ}$ 593)

In Herb.: Grotingen, Strat. (v. vo et s.) 


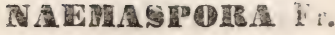

35.13. IN. errocea Pers, Op rottende schors ran boomen。 Aut. Vere. (Fr. Syst. III. p. 479; Rabenh. p. 41 ; Dz. et II b. Bijdr. 1. p. 30); Hyxosporium croceum Corda.

Mlaastricht, Franq.

3351 $\mathbb{1}$. microspora Desmaz, Op doode Populiertronken. Hieme. (F r. Syst. HI. p. 479 )

In ILerb.: Zuid-Beveland (Goes), vo d. B.; Maastricht, Franq. (v. v. et s.)

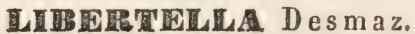

Receptaculum nullum. Sporidia fusiformia, reeta aut magis minusve curva massa gelatinosa juncta, sub epidermide plantarum mortuarum effusa, in cirros prodientia. (Desmaz. Monoggr. du G. Naem. in Mém. Soc. Roy. des Sc. de Lille 1829-30. p. 191)

3515. W. Detulima Desmaz. Op schors van afgestoryen tronken van Betula alba. Hieme.

Sporidiis aurantiaco-rubris, fusiformibas, subreclis, circiter 1/70 mill. lonģis. (Desmaz. l. l. p. 192. Tab. VI. f. 4 )

In Herb.: Zuid-Beveland, v. d. B. (v. v. et s.)

3516. L. faginea Desmaz. Op afirestorven Beukentronken en takken. llieme. Vere.

Sporidiis aurantiacis, fusiformibus curvissimis tenuissimis, circiter $1 / 60^{\circ}$ mill. longis, (Desmaz. I. I. p. 192. Tab. 6. fig. 5; Desmaz. Pl. Cr. de Fr. n $^{\circ}$ 707)

In Herb.: Amsterdam, v. de S. Late. (v. v. et so)

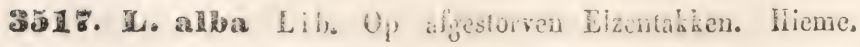
Vere.

Acervi rotundi; palpa atias in cimos erassus 
concolores erumpente; ascellis fusiformibus curvatis; sporidiis globosis pellucidis. Lib. Pl. Cr. Arduenn. no. 364)

In Ilerb.: Utrecht, Wttw. (v. vo et s.)

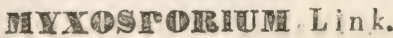

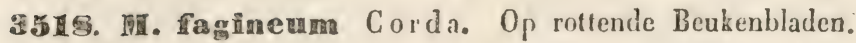
Ilieme. (R abenh. p. 41 ; Illosporium fagineum $L \mathrm{i}$. PI. Cr. Ard. no. 185)

Maastricht, Fran q.

$$
\text { * Ere of in el. }
$$

\section{Triำ.}

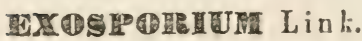

3519. W. thas Link. $0 p$ doode Lindetakken. Hieme. Vere. (Rabenh. p. 33; Ilelminthosporium tiliae Fr. Syst. III. p. 360)

In Herb.: Leiden, 0 u dem. (v. s.)

3520. W. Haypoderminm Link. Op doole stengels van groote kruidgewassen. Hieme. Vere. (R a be n h. p. 33) Maastricht, Franc.

3521. E. Hi⿱sppidula Link. Op bladen, halmen en aren van verschillende grassoorten. Aut. Hieme. (Rabenh. p. 33 ; Conoplea hispidula Lirk. non Pers.)

Maastricht, Franq.

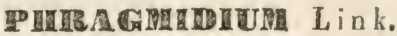

3522. IPhr, incrassatum $\mathrm{Link}, 0 p$ levende bladen van verschillende Rubus-soorten. $\Lambda$ ut. (R abenh. p. 32; Aregoma Phragmidium Tr。; Dz. et Ml b. Bijdr. I.p. 31; Puceinia mueronata l'ers.). Variat: 
a. Rosarum Wa Irs Op baden van eenige Rozen:

In Herb.: Amsterdam, v, d. S. Lac. - Bovendien: Mhaastricht, Franq. (v. v. et s.)

b. Ruborum Wallr. Puccinia rubi Schum.

In Ilerb.: Amsterdam, v. d. S. Lac. - Bovendien: Maastricht, Franq. (v. vo et 8. )

3523. Eher。 ohtusum Schm. et. Kze. Op bladen en bladstelen van cenige Potentilla-soorten. Aut. (Rabenh. p. 32)

In lierb.: Dongen in Noord-Braband. (v. v. et s.)

3524. Phr. apiculatum $\mathrm{R}$ abenh. Op bladen van $P_{o}$ terium-en Sanguisorba-soorten. Aut. (R a be n h. p. 32) Haastricht; Franq.

3525. F⿸耳又, asperum W a ll r. Op bladen van Rubus fruticosus. Aut. (R a benh. p. 32)

In Ilerb.: Amsterdam, v. d. S. L a c. (v. v. et s.)

3526. IPh. bullatum W est. Op stervende wilde Rozentakken in bosschen. Aut.

Taches épicaules, gris-jaunâtres, de 1 à 4 ou 5 cent. de longueur, limitées par une aréole pourprée, couvertes de boursouflures ou de petites ampoules inégales, produites par le soulèvement de l'épiderme. A la maturité, celle-ci se déchire irrégulièrement et laisse à nu, en larges coussinets noirs, des sporidies brun-rougeâtres, cylindriques, à 5,6 ou 7 cloisons et terminées par une petite pointe. Pédicelle hyalin, filiforme, epaissi vers le bas. (West. 4e Not. Cr. inéd. fl. B. p. 11. $n^{0}$. 44; W e 8 t. Herb. Crypt. B. $\left.\mathrm{n}^{0} .1069\right)$

In Herb.: Amsterdam, v. d. S. La c. (v. v. et s.)

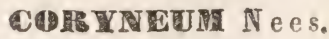

3528. Q. Uนmbonatum $\mathbb{N}$ es. Op doode Olmtakken. Hieme. 


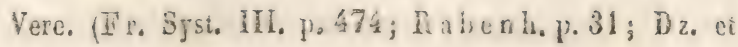
II b. Bijdr. III. p. 12).

In Herb.: Leiden, $D_{z_{0}}$ et $\mathrm{II} \mathrm{b}$; 0 ude m. (v. s.)

3529. C. pulluinatuan $\mathrm{Kz}$ e et $\mathrm{Schm}$. Op doode Lindetaklen. Ilieme. Vere. (Fr。 Syst. III. p. $474 ; \mathrm{R}$ a benh. p. 31)

In Herb.: Leiden, $D_{z}$. et $\mathbb{H} b_{\text {. }} ; 0$ adem.; W $t \mathrm{tw}$; Utrecht, Amsterdam, v. d. S. Lac. - Bovendien: Haastricht, Franq. (v. vo et s.)

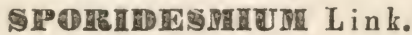

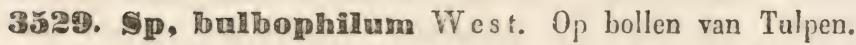
Aut. Hieme. (W est. Se Not. Crypt. inéd. fl. Belge pag. 11. $\left.n^{\circ} .35\right)$

Taches étalées, noires, indéterminées. Spores solitaires ou agrégése diaphanes, brunâtres, oblongs ou pyriformes, à trois cloisons et mesurant $3 / 100^{\text {es }}$ de mill. de longueur sur $1 / 100^{\circ}$ de mill. de largeur. Pédicclles Irès courts ou nuls.

In Herb.: Utrecht, Haarlem, Dzo et Mi b. (vov. et s.)

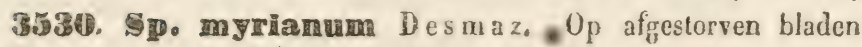
van Psamma arenaria. Aut. li ieme.

Cauli- vel foliicolum. Acervulis crumpentibus, minu* tissimis, innumerosis, approximatis, punctiformibus. Stromate tenui, convexiusculo, nigro; sporidiis congestis, fuscis semiopacis, polymorphis, ellipsoideis, obovatis vel pyriformibus, sessilibus aut brevissime pedicellatis, tri-vel tetradymis cellulosis. (Des maz.20e Not. Cr. de Fr。 p. 3. no 4 ; Desmaz, PI. Cr. de Fr. Nouv. Série $n^{\circ}$. 2)

In Herb.: llollandsche duinen.

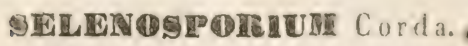

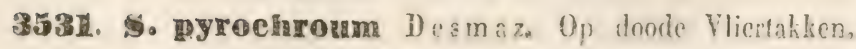
Ilieme. Vere. . 
Erumpens, sparsum, minutum, rolundatum, oblongum vel polymorphum (non caespitosum) ochroleucum, siccum flammeum. Stromate pallido vesiculoso. Sporidiis fusiformibus, acutis, curvatis, hyalinis, 3 septatis. Septulis aegre conspicuis. (Desmaz. 18e Not. Cr. de Fr. p. 5 ; Desmaz. Pl. Cr. de Fr. no. 1847)

In Herb.: Amsterdam, v. d. S. Lac. (v. s.)

3532. S. sarcochroum Desinaz. Op doode takjes van Cytisus laburnum. Hieme. Vere.

Erumpens, sparsum, minutum, tuberculariaeforme, ochroleucum, siccum carneum. Stromate convexo pallido; sporidiis majusculis, fusiformibus, acutis, plerumque curvatis, hyalinis, 5 raro 3 septatis; septis distinctissimis. (Desmaz. 18 e Not. Cr. de Fr. p. 6)

In Herb.: Leiden in 's Rijks Plantentuin, W $t \mathrm{tw}$. (v. v. et s.)

3533. asperifollorum West. Op kwijnende bladen van Symphytum officinale. Aut.

Taches très petites, 2 mill. au plus, arrondies, blanchâtres ou brunâtres. Strome très mince, blanc, étalé sur le centre de la tache. Sporidies hyalines, dressćes, filiformes, à extrémilés effilées; droites ou courbes, courtement pédicellées, les plus grandes incsurant $1 / 25^{\circ}$ de mill. de longueur sur $1 / 600^{\mathrm{e}}$ de mill. de lar-

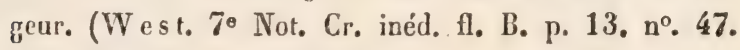
T. 1. fig. 2)

In Herb.: Kampen, R. Bond. (v. vo et s. $_{\text {) }}$

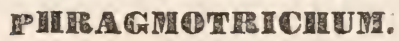

35318. Fhr. bullaria $\mathrm{C}$ or da. Op doode stengels van Angelica sylvestris. Aut. (R a ben h. p. 40; Didymosporium bullatum Fr. Syst. III. p. 487)

In Ilerb.: Kampen, R. B ond. (v. vo et s.) Bovendien: Maasiricht, $\mathrm{Franç}$, 


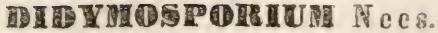

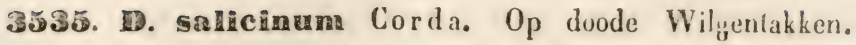
Ilieme. Vere. (Cord. Ic. fung. I. p. 5. T. II. f.108; Rabenh. p. 27)

In Herb.: Leiderdorp, (v. vo et s.)

3536. Deeris Desmaz. Op levende bladen van Acer campestre. Aest. Aut. (Desmaz. Pl. Crypt. de Fr. n。. 777; Cheilaria aceris Lib. Pl. Crypt. Ard. no. 255)

Maculis orbiculatis griseo-fuscis; peritheciis? minutis, rotundato-oblongis, nigris, rima dehiscentibus; pulpa fusca, ascis oblongis; sporidiis 2 pellucidis. (Lib. I. I.)

In Herb.: Haastricht, Franq. (v. vo et s.)

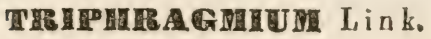

353\%, Tr. ulmariae Link. Op levende bladen van Spiraea ulmaria. Aut. (R a benh. p. 28)

In Herb.: Zuid-Beveland, $\nabla$. d. B. ( $\left.\nabla_{\text {. }} \nabla_{\text {。 et }} s\right)$.

\section{TUCCINIA Per8.}

3535. Traminis Pers. Op bladen en halmen van verschillende grassoorten. Aut. (Rabenh. p. 21; Dz. et MI b. Bijdr. I, p. 31), Variat:

a. epicaula West. $\mathrm{O}_{\mathrm{p}}$ doode grashalmen.

In Herb.: Amsterdam, v. d. S. Lac. (v. v. et s.)

b. epiphylla West. Op bladen van Phragmites communis.

In Ilerb.: Kampen, R. B on d.; Amsterdam, v. d. S. L a c. (v. v. et s.) - Bovendien: Maastricht, F r a $\mathrm{c}$.

3539. IP. arundinacea $H \mathrm{ed}$ w. f il. $0_{p}$ bladen en hal men van Phragmites communis. Aest. Aut. ( $R$ a b e $n$ h. p. 22; P. Striola Dz. et MI b. Bijdr. I, p. 31?) 
a. epiphylla Wallr. Comp. fl. Germ. IV. 225, Op de bladen.

In Herb.: Ubbergen, A bel. (v. vo et s.)

b. epicaula Wallr. I. l. Op de halmen.

In Herb.: Ubbergen, A bel. (vo vo et s.) - Bovendien: Maastricht, Franq.

3550. IP. caricis Dec. Op bladen van Carex laevigata en andere Carex-soorten. Aest. Aut. (R abenh. p. 22. Pucc. striola Link)

In Herb.: Zuid-Beveland, vo d. B. (v. vo et s.)

3541. IP. Scirpi Link. $0 p$ halmen van Scirpus lacustris. Aut. (Rabenh. p. 22)

Maastricht, Fran q:

3542. IP. allorum $\mathrm{Corda} 0 p$ de stengels van Allium vineale. Aut.

Caespitulis grregariis epidermide semper tectis, bullatis, fuscis; sporis oblongis, rarius ovoideis, fuscis, supra rotundatis vel obtusis, cellulis subaequalibus; episporio tenui amoene fusco; nucleo pallido; stipite filiformi, cavo, longo. (Cord a Ic. fung. IV. p. 12. T. III. f. 31)

In Herb.: Amsterdam, v. d. S. Lac. (v. v. et s.)

3543. ㄹ. polygonoram Schlech t. Op stengels en bladen van verschillende Polygonum-soorten. Aut. (Rabenh. p. 22; Dz. et II b. Bijdr. I, p. 31). Variat:

a. Bistortae Rabenh. 1. I. Op bladen van Polygonum, bistorta.

b. Amphibii Raben h. 1. 1. Op Polygonum amphibium.

c. Convolvuli Rabenh. I. 1. Op Polygonum convolvulus.

Maastricht, Franq.; Twello, Cop in herb。 Dav.

354 1. If. glechomatis Dec. Op bladen van Glechoma hederacea. Aut. (R a b e n h. p. 23; D z. et II b. Bijdr. I, p. 32)

In Herb.: Zwijndrecht, v. d. S. Lac. (vo vo et s.) 


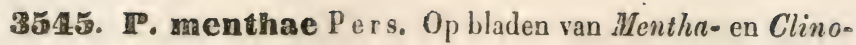
podium-soorten. Aut. (Rabenh. p. 23; D z, et $\mathbb{I}$ b. Bijdr. I, p. 32)

Naastricht, Fran q.

3546. P. compositarum Schltdl. Op bladen van Sonchus oleraceus. Aut. (Rabenh. p. 24; Dz. et M b. Bijdr. I, p. 32)

Maastricht, Fran q.

3548. discoidean $\mathrm{Link}$. $O_{p}$ bladen van Tanacetum vulgare Aut. (Rabenh. p. 24; Dz. et II b. Bijdr. I, p. 32) Maastricht, Franq.

354S. P. conglomerata $S \mathrm{chm}$. et $\mathrm{Kze}$. Op bladen van Centaurea scabiosa. Aut. (Rabenh. p. 24) Maastricht, Fran q.

3549. Paillantiae Pers. Op bladen van Vaillantia cruciata. Aut. (Rabenb. p. 25)

Mlaastricht, Fran q.

3550. TP. galiorum Link. Op bladen en stengels van Galium-soorten. Aut. (Rabenh. p. 21; Dz. et Hib. Bijdr. II, p. 21) Maastricht, Fran q.

3551. Pambelliferarum Dec. Op bladen van Apium graveolens en andere Umbelliferae. Aut. ( $\mathrm{R}$ abenh. p. 25; Dz. et $M$ b. Bijdr. I, p. 32)

Mlaastricht, Fran q.

3552. IP. aegopodia $L$ ink. Op bladen en bladstelen van Aegopodium podagraria. Aut. (R a be n h. p. $25 ; \mathrm{Dz}$. et II b. Bijdr. I, p. 32)

In Herb.: Amsterdam, v. d. S. La c. (v. v. et s.) Bovendien: Maastricht, Fran q.

3553. pimpinellae Link. $0_{p}$ bladen van Pimpinella saxifraga. Aut. (kabenh. p. 25)

Maastricht, Franq. 
3554. IP. aloxac Dec. Op bladen on bladstelen van Adoxa moschatellina. Aest. (R a b e n h. p. 25 ; P. saxifragarum $\mathrm{Dz}$. et $\mathrm{MI}$ b. Bijdr. III, p. 12)

Maastricht, Franq.

3555. P. saxifragarum Schltdl. Op bladen van Saxifraga-soorten. Aut. (Rabenh. p. 25)

Maastricht, Franq.

355. H. prunorum Link. Op Pruimenbladen. Aut. (R abenh. p. 26; Dz. et Ml b. Bijdr. I, p. 32)

Maastricht, Fr an q.

355\%. Pr. anemones Pers. Op bladen van Anemone nemorosa. Aest. Aut. ( $\mathrm{R}$ abenh. p. $26 ; \mathrm{D}$ z. et $\mathrm{M} \mathrm{b}$. Bijdr. I, p. 32)

In lierb.: Ubbergen bij Nijmegen, $\mathrm{A}$ bel (v. v. et s.)

3ร5ร8. FP. Stellariae Rabenh. Op bladen van Stellaria media en trinervia. Aut. (R a benh. Herb. viv. myc. edit. 2a $\mathrm{n}^{0} 86$; Pucc. lychnidearum $\mathrm{R}$ a benh. Deutschl. fl. I. p. 26; Dz. et $\mathrm{NIb}$ b. Bïjr. I, p. 32)

. In Herb.: Zwijndrecht, v. d. S. La c. (v. vo et 8.) Bovendien: Mlastricht, Franq.

35559. EP. dianthi Dec. Op bladen van Dianthus barbatus. Aut. (Pucc. lychnidearum pr. part. Rabenh. 1. I. p. 26; Dz. et $\mathrm{II}$ b. Bijdr. I, p. 32)

In Herb.: Zuid-Beveland, v. d. B. (v. v. et s.)

35010. Pabae Link. 0 p stengels van Vicia faba. Aut. (Rabenh. p. 27)

Maastricht, Franq.

3561. P. cirsill Las $\mathrm{ch}$. Op bladen van Cirsium arvense. Aut. (Lasch, in Rabenh. Fung. Eur. ${ }^{\circ} 89$ )

In Herb.: Fort Sterrenschans hij Nijmegen, $\mathrm{A}$ bel. (v. v, et $s_{0}$ )

:362. P. sonchin Rob. Op drooge stengrels van Sonchus arvensis on oleraceus. Ant. 
ITaculis fusco-rubescentibus vel nullis; acervulis h̀ypurarius epiphyllis vel caulinis, epidermide tectis, numerosis, rotundatis vel oblongis, nigrris, subnitidis, rugulosis, approximatis vel circulariter positis, saepe confluentibas et crustaceo effusis; sporidiis fuscis, oblongis, medio contractis, pediccllo subaequali. ( $\mathrm{Rob}$. in $\mathrm{Des-}$ maz. 170 not. Cr. de fr. p. 2. no 3 ; Desmaz. P!. Cr. de fr. $n^{0}$ 1831)

In Herb.: Zuid-Beveland (Goes) v. d. B. (v. v. et 8.)

NB. Praeter species hic memoratas indigenae enu= merantur in Dz. et II b. Bijdr. I, p. 31 et $32: P$. Calthae Lk., P. Gentianae Lk.; $\boldsymbol{P}$. Aethusae Lk., P. Violarum Lk., P. Circaeae P.; P. Liliacearum Dc., $P$. Allii Desm:

\section{Trib. Uredimeil genuini.}

\section{PEIRIDERMIUM L in $\mathrm{k}$.}

3563. IP. pini $\mathrm{W}$ all r. Op doode bladen van verschillende Pijoboomen. Aest. Aut. (R a ben h. p. 21; $\mathrm{D}_{z_{\text {s }}}$ et $\mathrm{Ml}$ b. Bijdr. II, p. 21).

In Herb.: Zuid-Beveland, v. d, B. ( $v$, vo et s.)

\section{IROERTELIA $\mathrm{Rabenh}$.}

35G1. R, camcellata ( $\mathrm{Linn}$.) $\mathrm{R}$ a benh. Op bladen van Appelen en Peeren. Aut. (Rabenh. p. 20; Caeoma roestelites $\mathbf{D} z$, et $\mathrm{M} \mathrm{b}$. Bijdr. I, p. 37).

In Herb.: Zuid-Beveland (Goes), v. d. B. (v, v. et s.) Bovendien: Maastricht, Fra $\mathrm{n}$.

3565. H. Hacerada Mer. Op bladeu van Crataegus monogyna. Aut. (Aecidium cornutum a oxyacanthae Rabenh. p. 20; Caeoma cylindrites Dz. et Ml b. I, 1. 37)

In Herb.: Utrecht, vo d. S. Lac. (v. vo et s.) Bovendien: Blaastricht, Franq. Variat: 
6. Mespili $\mathrm{K}$. $U_{\mathrm{p}}$ bladen van Mespilus germanica. Aut. (Aecid. cornulum e. mespili R a be n h. l.1.)

In Herb.:? - Bovendien: Ilaastricht, Franq.

CIDNARTMR (Kunze) Fries。

3516. Cr. asclepiadeum $\mathrm{Fr}$. $O_{p}$ bladen van Cynanchum vincetoxicum. Aest. Aut. (R abenh. p. 20)

a. Paeoniae Fr. Op bladen van Paeonia officinalis. Aut. (Sphaeria flaccida. Alb. et Schw.; Fr. Syst. Il. p. 516; Rabenh. p. 172)

In Herb.: Naaldwijk, v.d. Tr.; Rotterdam, 0 a de m. (v. v, et ss)

\section{A ECIDEUPR Pers.}

356\%. Aec. adozae Grav. Op bladen en bladstelen van Adoxa moschatellina. Aest.

Caulinum bifronsque, maculis nullis, pseudoperi* diis sparsis laxis pallide luteis urceolato-cupulatis, margine crecto aut subpatulo lacinulato deciduo, sporidiis globosis pallide luteis. (Grav. in Duby Bot. Gall. II. p. 908; Caeoma Adoxae Dz. et Mlb. Bijdr. III, p. 12). Maastricht, Franq.

3568. Aec. tussillaginis $\mathrm{W}$ all r. Op bladen van Tussilago farfara. Aest. Aut. (Aec. compositarum b. Tussilaginis Pers., Rabenh. p. 15; Caeoma Compositarum $D z$. et $\mathbb{I l}$ b. Bijdr. I, p. 36 , p. p.).

Maastricht, Franq.

3569. Aec. cichoracearum Dec. Op bladen van Scorzonera en Tragopogon-soorten. Aest. Aut. (Rabenh. p. 16; Cacoma Tragopogonatum $\mathrm{D}$ z. et $\mathbf{I l}$ b. Bijdr. I, p. 36 , p. p.).

Maastricht, Fran q.

3580. Aec. asperifolii Pers. Op bladen van Cynoglos. 
sum offieinale. Aut. (R a beni. P. 16; Cacoma Burraginatum $\mathrm{D} z$. et $\mathbf{M} \mathrm{b}$. Bijdr. I, p. 36). Maastricht, Fr an q.

3581. Acc. urticae $\mathrm{Sehum}$. $0 \mathrm{p}$ bladen van Brandnetels. Aut. (Rabenh. p. 16; Caeoma Urticatum D z. et ill b. Bijdr. I, p, 37).

Maastricht, Franq.

3582. Aec. grossulariae Dec. $0_{p}$. bladen van Ribessoorten. Aut. (R a benh. p. 16; Caeoma Grossulariatum Dz. et Ml b. Bijdr. I, p. 37)。

Maastrioht, Franq.

3583. Aec. violae $\mathrm{S} \mathrm{chum}$. Op bladen en bladstelen van Viola-soorten. Aut. (R abenh. p. 17; Caeoma Viola* tum Dz. et II b. Bïdr. I, p. 37).

Maastricht, Fran q.

3584. Aec. glancis $\mathrm{D} z$, et $\mathrm{II} b$. Op bladen van Glaux maritima. Aest. ( $\mathrm{D}_{\text {z }}$ et $\mathrm{Ml} \mathrm{b}$. Nov. fung. spec. in Tijdschr. v. nat. gesch. T. XII. p. 16 T. V. f. 1, 2, 3 et Bijdr. II, p. 21).

Zuid-Beveland, v. d. B.

3585. Aec. Heucospermum Dec. Op bladen van Anemone nemorosa. Aest. (R abenh. p. 18; C'aeoma leucospermatum Dz. et $\mathrm{II}$ b. Bijdr. I, p. 36).

In Herb.: Ubbergen bij Nijmegen, A b e l. (vo vo et s.) Bovendien: Maastricht: Fran q.

3586. Aec. ranunculacearum Dec. $O_{p}$ bladen en bladstelen van verschillende Ranunculaceeẽn. Aest. Aut. (R abenh. p. 18; Caeoma Ranunculaceatum Dz. et $\mathrm{II}$ b. Bijdr. I, p. 36). Variat:

a. Ranunculi Pers. Op bladen van Ranunculus-soorten. (R a benh. 1. I.)

Maastricht, Fran q.

b. Ficariae Pers. Op bladen van Ficaria ranunculoides. (R a ben h, I. I.)

Haastricht, Franq. 
c. Aquilegiac Pers. Op bladen van Aquilegia vuigaris. (R abenh. p. 19)

Maastricht, Franq.

d. Clematidis Dec. Op bladen van Clematis vilalba.

Maastricht, Fran q.

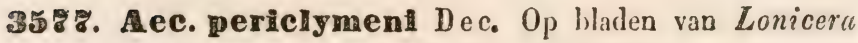
periclymenum. Aut. (Raben l. p. 19)

Mlaastricht, Fr anq.

3588. Aec. falcariae Dec. Op bladen van Sium falcaria. Aut. (Rabenh. p. 19)

IJaastricht, Franq.

35 verschillende Leguminosae. Aut, ( $\mathrm{R}$ abenh. p. 10) Variat:

a. Trifolii Rabenh, l. l. Op Klaverbladen. Mlaastricht, Fran q.

3550. Aec. Thamni Pers. Op bladen, bladstelen en jonge takjes van Rhammus frangula. Aut. (Acc. elongatum a. Rhamni Rabenh. p. 19; Caeoma crassatum et Rhamnatum $\mathrm{D} z$. et $\mathrm{II}$ b. Bijdr. I, p. 37).

Maastricht, Fran q.

3581. Aec. berberidis Pers. Op bladen van Berberis vulgaris. Aut. (Aec, elongatum $d$. berberidis $\mathrm{R} a$ benh.p. 20; Cacoma Berberidatum Dz. et II. Bijdr. I. p. 37).

IIaastricht, Fran q.

3582. Aec. phaseolorwm $\mathrm{W}$ allr. Op bladen van Phaseolus vulgaris. Aut. ( $\mathrm{R}$ a ben h. p. 20)

In Herb.: Beuningen, A bel. (v. v. et s.)

3583. Acc. convallarlae $\mathrm{Sch}$ u $\mathrm{m}$. $O p$ bladen van verschillende soorten van Convallaria. Aut. (Rabenh. p. 15; Cacoma Convallariatum $\mathrm{D} z$, et $3 \mathrm{I} b, \mathrm{Bijdr}$. I, 3. 36). 
In Herb.: Groningen, Strat.; Ubbergen bij Nijo meggen, A bel. (v. v. et s.)

3581. Ace. myrtilli Franq. $0 p$ de onderzijde der bladen van Vaccinium myrtillus: Aut.

Sporen gelb; Sporidien klein in Kreisen lange verschlossen, endlich ausbrechend; Saum kaum zurïckgeschlagen. (Fran q. Hss, )

Haastricht, Franq.

3585. Ace. pyri Franq. Op Peerenbladen Aut.

Sporen orange; Sporidien klein, etwas eingesenkt, in unregelmässigen Flecken disponirt anf der Oberfläche des Blattes, gleichfarbig, spät zerrisscn mit zurückireschlagenem Saume. (Franq. Mss,; non Roestelia cancellata nec Aec. cornutum)

Haastricht, Franq.

3596. Aec, seneciomis Desmaz, Op bladen van $S e$ necio aquatica en Jacobaea. Aut.

Maculis flavescentibus. Peridiis hypogenis, subconcentricis aut coufertis. Ore dentato, primum contracto, demum patulo et reflexo. Sporulis aurantiacis. (Desmaz. Pl. Cr. de fr. no 677; Desmaz. Hém. Soc. Roy. des Sc. de Lille 1834. p. 14)

In Herb.: ? . . ( (v. v. et s.)

Speciebus enumeratis accedunt: Caeoma Alliatum Lk., C. rubellatım Lk., C. Melampyratum Kze., C. Buniatum L k., C. quadrifidum L k., C. Epilobiatum Lk., C. Euphorbiatum L k., C. Salicorniatum $\mathrm{Lk}$., $C$. Ari $\mathrm{Dz}$. et $\mathrm{M}$ b., C. IIeraclei $\mathrm{Dz}$. et $\mathrm{Ml} \mathrm{b}$. (in Dz. et $\mathrm{HI} b . \mathrm{Bijdr} . \mathrm{I}, \mathrm{II}, \mathrm{III}$ ).

\section{CON IOTHECIU⿴囗十⺝ Corda.}

358\%. C. amentacearum Corda. Op afirestorven takjes van Wilgen in de duinen. Aut. llieme. (Corda Ie fung. 1. p. 2. T. 1. fir 26 ; R a benh. P. 15) 
In Ilcrb.: Leiden, Wtiw.; Amsterdam, v. d. S. L a c. (v. v. et s.)

3585. C. conglutinatum $\mathrm{C}$ orda. $0 p$ ontschorste doode takken. (Corda Ic. fung. I. p. 2. T. 1. fig. 20; Rabenh. p. 14)

In Herb.: Leiderdorp. (v. s.)

3559. C. effusum Corda. $O_{p}$ vochtig rottend hout. Hieme. Vere. (Corda Ic. fung. I. p. 2. Tab. 1. f. 21; Rabenh. p. 14)

In Herb.: Leiden, $0 \mathrm{ade}$ m. (v. s.)

3596. C. phyllophillum Desmaz. Op verdroogde Likenbladen. Hieme.

Hypophyllum, atrum, minutum, tenue maculaeforme. Sporulis conglutinatis, minutissimis, globosis, fuseis, semihyalinis. (Desmaz. 11e Not. Gr. de fr. p. 358; Desmaz. PI. Cr. de fr. $n^{\circ}$ 1427)

In Herb.: Ansterdam, 0 u de m. (v. s.)

3591. C. betulinum $\mathrm{C}$ orda. $0 p$ doode takken en takjes van Betula alba. Aut. (Corda Ic fung. I. p. 2. T. 1.f. 25) In Herb.: Achttienhoven, v. d. S. Lac. (v. v. et s.)

CONISPOHEUTI L in $\mathrm{k}$.

3592. C. buxi West. $O p$ doode afgevallen takken van Buxus sempervirens. Vere.

Effusum, fusco-nigrum. Sporis ovoideis vel pyriformibus, pedicellatis, rufo-fuscis, impellucidis, longis $1 / 100$ ad $4 / 100$ mill, - Pedicello brevissimo, hyalino. (W e s t. $8^{\mathrm{e}}$ not. Crypt. inéd. fl. B. pag. 11. $\mathrm{n}^{\circ} 36$ fig. $7,0, p_{0}$ )

In Herb.: Utrecht, W $t \mathrm{tw}$. (v. v. et s.)

UUE

* Urédinnées sans Cystides.

Réceptacle caché sous l'épiderme; spores s'échappant 
sous furme de poussière. (Lév. Dispos. méth. des Uréd. in Ann. des Sc. nat. 3 Sér. T. VIII)

$1^{0}$ Réceptacle composé d'un tissu à mailles irrégu. lières, à peine distinctes, formant un petit coussinet, de la surface duquel naiszent les spores; spores simples, gुlobuleuses, pédicellćes, entrainant avec elles leurs jédicelles ou s'en détachant. (Uromyces Lév. I. 1.)

\section{A. Spores jaunes ou orangées.}

3593. U. Tubigo-vera $\mathrm{Dec}$. $\mathrm{Op}_{\mathrm{p}}$ bladen en halmen vau granen en andere grassoorten. Aest. (Rabenh. p. 13; Caeoma Rubigo Dz. et II b. Bijdr. I, p. 33).

In Herb.: Utrecht, W $t \mathrm{tw}$. (v. v. et s.)

3594. U. Thinanthacearum $\mathrm{D}$ e c. 0 p bladen van Rhinanthus, Melampyrum, enz. Aest. (Raben h. p. 10; Caeoma Rhinanthacearnm Dz. et Mb. Bijdr. I, p. 33), Variat:

a. Euphrasiae R aben h. I. 1. Op bladen van Euphrasia odontites.

In Ilerb.: Amsterdam, v. d. S. Lac. (v. v. et s.)

b. Melampyri Rabenh. Op bladen van Melampyrum arvense.

In Herb. Utrecht, W $t \mathrm{tw}$. (v. s.)

3595. U. alchemillae Pers. Op bladen van Alchemilla vulgaris. Aest. (Rabenh. p. 10)

Hlaastricht, Franq.

B. Spores rousses, brunes ou noires.

3596. U. leguminosarum Desmaz. Op bladen van verschillende soorten van Leguminosae. Aest. Aut. (Rabenh. p. 7; Caeoma Trifolii, C. appendiculatum et C. Legguminosarum D z et $\mathbb{B}$ b, Bijdr. I, p. 35 et II. p. 21). 
๑. Ficiarum Rabenh. I. I. Op Vicia sepium. Mlaastricht, Franq.

b. Trifoliorum R aben h. I. I. Op Klaverbladen. (U. apiculosa v. Trifolii Berk.)

In Ilerb.: Zuid-Beveland, v. d. B.; Leiden, D z. et $1 I$ b. (v. v. et S.) - Bovendien: Maastricht, Franq.

c. Fabarum Raben h. 1. I. Op bladen van Vicia faba. (Uredo Viciae fabae Dec.)

In Herb.: Leiden, $\mathrm{W} t \mathrm{t}$ w. (v. vi et 8.)

d. Phaseolorum Rabenh. 1. I. Op bladen van Phaseolus vulgaris, in moeslanden. (U. Phaseolorum $\mathrm{Dec}$.)

In Ilerb.: Amsterdain, v. d. S. L a c.; Kampen, R. Bond. (v. v. et s.)

359\%. ש. Heguminum Desmaz. Op de peulen van Phascolus en Vicia faba. Aut.

Acervis rotundis, solitariis, maximis, epidermide rupta cinctis. Sporulis ovoideis, pedicellatis, fuscis. (Desmaz. Pl. Cr. de Fr. no. 934; Desmaz. Ilém. Soc. Roy. des Sc. de Lille $18391^{\circ}$ partie p. 78. $n^{\circ} .5$ )

In Herb.: Steeg bij Nijmegen, Abel. (v. v. et s.)

3598. ש. apiculata Strauss: $0 p$ bladen van Cytisus laburnum: Aut. (R a b e n h. p. 4; Ur. Cytisi laburni Dec.; Caeoma apiculatum $\mathrm{D} z$. et $\mathrm{II}$ b. Bijdr. I, p. 35).

Leiden, Dz. et $\mathrm{II} \mathrm{b}$.

3599. U. polygonorum Dec. Op bladen van Polygonum aviculare. Aut. (R a benh. p. 6 ; Caeoma Polygonorum $\mathrm{Dz}$. et $\mathrm{HI} b$. Bïdr. I, p. 33).

In Herb.: Zuid-Beveland (Goes), v. d. B. (v. v. et s.) Bovendien: Maastricht, Franq.

3600. ש. epilotii Dec. Op bladen van Epilobium-soorten. Aest. Aut. (Rabenh. p. 8; Cacoma Epilobii D z, et II b. Bijdr. II, [1. 21). 


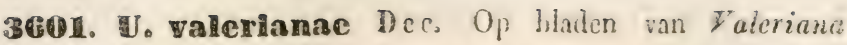
officinalis. Mest. Aat. (Rabenh. p. 9; Cacoma Valerianæ $\mathrm{D}$ z. et $\mathrm{MI}$ b. Bijdr. I, p. 35).

In Herb.: Leiderdorp. (v. s.) - Bovendien:' IIaastricht, Franq.

3602. U. ambigua Dec. Op bladen van Allium scorndoprasum. Aest. (Rabenh. p. 6)

Maastricht, Franq.

3603. U. gentianae $\mathrm{Dec}$ e $0 \mathrm{p}$ bladen van Gentiana pneumonanthe. Aest. (Rabenh. p. 5)

In Ilerb.: Vinkeveen, v. d. S. Lac. (v, vo et s.)

20. Réceptacles composés de très petites cellules irrégुulières, formant une sorte de plateau couvert de vésicules eylindriques, terminćes par plusicurs spores disposées en ehapelet; spores sphériques ou cubiques. (Cystopus Lév. I. I.)

3604. U. candida Pers. Op bladen, stengels en zaadhaisjes van verschillende kruidgewassen: Aest. Auf? (Rabenh. p. 13; Caeoma candidum Dz. et II b. Bijdr. I, p. 35), Variat:

a. Cruciferarum $\mathrm{R}$ abenh. $\mathrm{O}_{\mathrm{p}}$ Capsella bursa pastoris en Erysimum tenuifolium.

In Ilerb.: Amsterdam, 0 udem. (v. v. et s.) Bovendien: IIaastricht, Franq.

b. Compositarum Rabenh. 1. I. Op bladen van Seorzonera hispanica. Aest, Aut.

In Herb.: Zutphen. W $t \mathrm{tw}$. (v. v. et s.)

3605. U. portulacae $\mathrm{Dekin}$. $\mathrm{O}_{\mathrm{p}}$ bladen van Portulaca oleracea. Aest. (Dekin. fl. Brux.; U. candida c. Portulacearum Rahenh. p. 13; Cacoma candidum B. grossum Dz. et $1 I \mathrm{~b}$. Bijdr. I, p. 35).

In Herb.: Zutphen, W $t t w$. (v, v. et s.) - Bovendi n: Iaastricht, Fran q. 
3\%. Réceptacle composé de très petites cellules irrégulières, formant une sorte de plateau dont la surface est couverte de plusieurs assises de cellules inégales, renfermant chacune une spore; spores simples, toujours dépourvues d'appendices. (Uredo Pers.; L év. l. l.)

A. Spores jaunes ou orangées.

3606. U. symphyti Dec. $O_{p}$ de bladen van Symphytum officinale. Aest. Aut. (R abenh. p. 11; Caeoma Symphyti Dz. et II b. Bijdr. I, p. 35).

In Herb.: Culenborg, Leiden, W $t \mathrm{tw}$. ex herb. Arkenh. (v. vo et s.) - Bovendien: Maastricht, Franq.

3608. U. pustulata Pers. Op bladen van Cerastium en Stellaria-soorten. Aest. (U. Caryophyllacearum Rabenh. p. 11; Caeoma Caryophyllacearum Dz. et II b. Bijdr. I, p. 34). Variat:

a. Caryophyllacearum Pers. 0 p Cerastium vulgatum.

In Herb.: Leiden, W $t \mathrm{t}$ w. ex herb. A r k e $\mathrm{n}$ h. (v.s.)

b. Epilobiorum Pers. Op bladen van Epilobium spicatum.

In Herb.: Voordorp bij Leiderdorp. (v. vo et $s_{s}$ )

3609. U. potentillarum Dec. $0 p$ bladen van Potentilla en Agrimonia-soorten: Aest. Aut. (R abenh. p. 9; Cąoma Potentillarum et polymorpha $\mathbf{D} z$. et $\mathbb{M}$ b. Bijdr. I, p. 34).

Maastricht, Franq.

3609. U. padi $\mathrm{Kz}$ e et $\mathrm{S} \mathrm{chm}$. $\mathrm{Op}_{\mathrm{p}}$ bladen van Prunus padus. Aut. (R abenh. p. 12)

Maastricht, Franq.

3610. U. sonchi Pers. Op bladen van Sonchus-soorten. Aest. Aut. (U. fulva a. Sonchorum Rabenh. p. 12 ; Caeoma Compransor Dz. et M b. Bijdr. I, p. 34).

In Herb.: Leiden, Wttw. ex herb. Arkenh. (v. si) - Bovendien; Haastricht, Franq. 
3611. U. Cacallae Dec. Op haden van Cacalia alpina. Aest. Aut. (Rabenh. p. 12; Caeoma Comprausor Dz. et II b. Bijdr. I, p. 34 , p. p.).

Maastricht, Franq.

3912. U. linearis Pers. $0 p$ bladen en halmen van granen en andere grassoorten. Aest. Aut. (R abenh. p. 13; Caeoma lineare Dz. et II b. Bịjdr. I, p. 33).

In Herb.: Leiden, W $t \mathrm{tw}$. ex herb. Arkenh. (v. v. et s.) - Bovendien : Maastricht, Franq.

3613. U. senecionis Schum. Op bladen van Senecio jacobaea. Aut. (Rabenh. p. 12; Cacoma Senecionis D z. et Mlb. I, p. 34).

In Herb.: Leiden, Wttw. ex herb. Arkenh: (v. v. et s.) - Bovendien: Maastricht, Franq.

3614. U. sempervivi $A$ lb. et Schw. $0 p$ bladen van Sempervivum tectorum. Aut. (R a ben h. p. 9 ; Cacoma Sempervivi Dz. et II b. Bijdr. II, p. 21). Haastricht, Franq.

3615. ש. fallens Desmaz. Op Klaverbladen. Aest.

Haculis obliteratis. Acervulis hypo- rarius epiphyllis, numerosis, sparsis, subrotundis, rufis, epidermide rupta cinctis. Sporulis subovatis, pedicello albo, brevi instructis, episporio tenuissime verrucoso. (Desmaz. $11^{\mathrm{e}}$ Not. Cr. Nouv, de la fr. p. $357 \mathrm{n}^{\circ} 1$; Desmaz. Pl. Cr. de Fr. $n^{\circ}$ 1325)

In IIerb.: Leiden, langs het Marendijkje, 0 ude m. (v. v. et s.)

3616. Uetae Pers. Op bladen van Beta vulgaris. Aest. Aut. (Rabenh. p. 6; Caeoma Betarum Dz. et II b. I, p. 33).

Maastricht, F ranq.

3618. U. preoniarum Desmaz. Op haden van Paconia officinalis, dikwijls gemengd met Cronartiuin asclepiadeum a, paeonine. Aut. 
Maculis amphigenis, irregularibus, hunneo-violaceis. Acervulis hypophyllinis, numerosis, orbicularibus ovalibusve dein confluentibus, pallide aurantiacis epidermide bullata tectis, Sporulis ovoideis vel pyriformibus. Episporio duplici subhyalino, tenuissime verrueso, nuclco brunneo granuloso. (Des maz. 14e Not. Cr. inéd. de la Fr. p. 11. no. 4; Desmaz. Pl. Cr. de Fr. $n^{\circ}$. 1664)

In Ilerb.: Rotterdam in den Ilortus Botanicus; O ud e m. (v. v. et s.)

B. Spores faures, brunes ou noires.

3618. ש. staveoleas Pers. Op bladen van Serratula arvensis. Aut. (Rabenh. p. 6; Caeoma suaveolens D z. et II b. Bijdr. I, p. 34).

In Herb.: Utrecht, W $t \mathrm{tw}_{0}$ - Bovendien: Haastricht, Franq.

3619. U. seirpil West. Op stengels van Scirpus lacustris. Aut.

Groupes d'un brun canelle, allongées, elliptiques ou linéaires, épars, recouverts par l'épiderme, qui se rompt longyitudinalement suivant les fibres du support. Sporidies brunes, globuleuses, pyriformes ou ovoides. (West. 7e Not. Cr. de la fl. B. p. 12. no 38 ; West. Herb. Cr. B. $\mathrm{n}^{\circ}$, 1266)

In llerb.: Amsterdam, vo d. S. L a c. (v. v. et s.)

3620. U, rumicum Dec. Op bladen van Rumex acetosa. Aest. (R a benh. p. 6; Caeoma Rumicum $\mathrm{D} z$, et $\mathbb{I I}$ b. Bịdr. I, p. 33).

Maastricht, Fran q.

3621. U. muricella $W$ allr. Op bladen van verschillende soorten van Umbelliferae. Aut. (Rabenh. p. 6; Cateoma Umbellatarum Dz, et Mb. Bijldr. I, p. 34). Variat: 
a. Cynapii Dec.; Rabenh. 1. 1. Op bladen van Aethusa cynapium.

Maastricht, Franq.

b. Conii Strauss, Op bladen van Conium maculatum. Maastricht, Fran q.

362. soorten van Labiatae. Aut. (Rabenh. p. 10). Variat:

a. Menthae Pers. Op Mentha-soorten.

Maastricht, Fran q.

b. Clinopodii Rabenh. Op bladen van Clinopodium vulgare.

Maastricht, Fran q.

3823. U. Alogeulosorum Al b; et $\mathrm{Sch}$ w. Op bladen van Apargia autumnalis. Aut. (Rabenh. p. 6; Ureảo apargiae Schleich.)

In Herb.: Utrecht, Dz, et Mb. - Bovendien: Maastricht, Franq.

3698. U. Dullatum West. Op stengels en somtijds op bladen van Dianthus prolifer. Aut.

Tâches épicaules, grisâtres, de 1 a 2 cent. de longueur, couvertes de boursoufflures inégales, produites par le soulèvement de l'épiderme; à la maturité celle ci se déchire longitudinalement et laisse à nu des coussinets orangés. Ces conssinets sont formés par des sporidies globuleuses, arrondies ou pyriformes, quelquefois pédicellées et mesurant environ $1 / 40$ de mill. de diamètre. (W e st. $8^{e}$ Not. Crypt. inéd. fl. B. pag. 9. no. 27)

In Herb.: Weurt bij Nịmegen, Gev. Deyn.? (v. vo et $s_{0}$ )

3685. U. epllobll Dec. Op bladen van Epilobium hirsutum. Aut. ( $\mathrm{R}$ a ben h. p. 8)

In Herb.: Leiden, W $t$ w. ex herb. Arkenh。 (v. v. et s.) - Bovendien: Maastricht, Fran q. 
4\%. Réceptacle composé de filaments rameax; ramifications supportant à leur extrémité un sporange formé de cellales polygonales, imitant un réseau vésiculeux autour d'une spore centrale; spores globuleuses ou ovales. (PPolyçstís Lé v.; Uroeystis Rabenh.)

3626. U. anemones Pers. Op bladen en bladstelen van Anemone nemorosa Aest. Aut. ( $\mathrm{R}$ a ben h. p. 4)

In Herb.: Leiden, $\mathbf{D} z_{\text {。 }}$ et $M b_{\text {。 }}$ (v, vo et s.)

3628. U. Colehici Link. Op bladen van Colchicum autum. nale. Ant. (Rabenh. p. 4. Sporisoriam colchici Lib.)

In Ilerb.: Kampen, R. Bond. (v. v. et s.)

3629. U. pompholygodes Schltdl. Op bladen van Helleborus viridis en Ranunculus bulbosus. Aest. Aut. (R a benh. p. 4; Ustilago pompholygodes Dzo et II b. Bijdr. II, p. 22).

Zuid-Beveland (Goes), v. d. B.; Maastricht, Franq. 3699. ש. ficariae $\mathrm{A} / \mathrm{b}$. et $\mathrm{Sch}$ w. Op bladen van Ficaria ranunculoides. Aest. (Rabenh. p. 5; Caeoma Ficariac Dz. et $I 1$ b. Bijdr. II, p. 21)

Maastricht, Franq.

5. Réceptacle composé de filaments rameus; ramifications terminées par une spore; spores simples. (Gilletia Tul.; Lé v. I. I.)

3930. W. caries Dec. In Tarwekorrels. Aest. (Uredo sitcphila Ditm.; Rabenh.p. 3; Cacoma sitophilum Dz。 et II b. Bijdr. I, p. 33)

In Herb.: Zutphen, W $\mathbf{t}$ w。 - Bovendien: Leiden, Dz, et Mb.

6. Réceptacle composé de filaments rameux; ramificaticns renflées au sommet, et supportant $8-20$ spores.

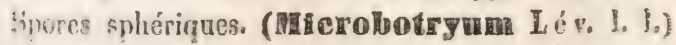


3631. U. violacea Pers. Op de helmknoppen van hialcchium aquaticum en Lychnis vespertina. Aest. Aut. (Rabenh. p. 3; Uredo antherarum Dec.; Ustilago antherarum Dz. et $\mathbf{M}$ b. Bijdr. I, p. 38)

Voorschoten, Dz, et $\mathbb{I} b_{0}$; Zuid-Beveland, vo d. B.

3632. U. zeceptaculorm $\mathrm{Dec}$. In den vruchtbodem van Scorzonera- en Tragopogon-soorten. Aest. (Rabenh. p. 4; Ustilago receptaculorum Dz. et II b. Bijdr. I, p. 37) Leiden, $\mathbf{D} z$, et $I I \mathrm{~b}$.

7. Réceptacle composé de cellules très petites, ir* régulières, donnant naissance à plusieurs couches dc cellules monospores (sporanges); spores globuleuses. (Ustilago Bauh.; Lé v. 1. 1.)

3633. U. segetum Pers. In de bloemstengels van Haver, Gerst en Tarwe. Aest. (R a benh. p. 4; Ustilaggo seģetum Dz. et $M$ b. Bijdr. I, p, 38). Variat:

a. Averae Pers. Op Ilaver en Arrhenatherum avenaceum.

In Herb.: Utrecht, W $\mathbf{t} \mathbf{t w}$ - Bovendien: Leiden, Dz. et $\mathrm{Mb}$.; v. HaIl. in Herb, De Vr. (v, vo et s.) b. Hordei Pers. Op Gerst.

In Herb.: Utrecht, W $\mathrm{t} t \mathrm{w}$. - Bovendien: ZuidBeveland, v. d. B. (v. v. et s.)

c. Tritici Pers. Op Tarwe.

In Herb.: Utrecht, W $t \mathrm{tw}$. (v. s.) - Bovendien: Leiden, Dz. et II b.; Mlaastricht, Franq.

3634. U. urceolorum Dec. Op kafjes en vruchtblaasjes van Cares acuta en arenaria. Aest. (U. caricis Pers.; Rabenh. p. 3; Caeoma caricis $\mathbf{D} z$. et M b. Bijdr. I, p. 33)

Bij Haarlem, Dz. et II b.

36ื5. ש. Oliwacea Dec. In den ruchtbodem van Carer areqaria. Acst. (Rahenh. p. 3)

Noordwịkerhout, Mb. 
3686. U. atriculosa D aby. In zaden van Polygonum Braunii en hydropiper. Aut. (R a be n h. p. 3 ; Caeoma utriculosum $\mathbf{D} z$, et $\mathrm{MI}$ b. Bijdr. I, p. 33)

Utrecht. v. d. S. Lac.; Maastricht, Fra aq.

363ร. บ. Hongisstma Sow. Op bladen van Glyceria aquatica. Aest. Aut. (Rabenh. p. 4; Caeoma longissimum Dz, et $\mathbf{l l}$ b. Bijdr. I, p. 33)

In Ilerb.: Utrecht, W $t \mathbf{t w}$. (v. s.) - Bovendien: Leiden, Dz. et II b.; de $\mathrm{H}_{\text {a a n }}$; Zuid-Beveland, v.d. B.

3638. T. hypodytes Rabenh. $O_{p}$ halmen van Elymus arenarius. Aest. Aat. (Rabenh. p. 4; Ustilago hypodytes Dz. et M b. Bijdr. I, p. 37)

Katwijk, Zaudvoort en Scheveningen, Dz. et M b. Variat :

a. Tritici Desunaz. Op halmen van Triticum repens.

In Herb.: Kampen, R. Bond. - Bovendien: ZuidBeveland, จ. d. B.

3639. đ. salveil Berk. et Broom. Op bladen van Dactylis glomerata. Aest. Aut.

Acervulis elongatis. linearibus, parallelis, saturate branneis; sporulis subovoideis, verruculosis, semiopacis, $1 / 100^{\mathrm{am}}$ inter et $15 / 1000^{\text {as }}$ mill. diametro variantibus. (Ustilago salveii Berk. et Broom.; Desmaz. 22。 Not. Crypt. inéd. de la Fr. p. 1. $n^{\circ}$ 1)

In Herb. : Leiden, D z. et II b.

8. Réceptacle composé de très petites cellules irrégulières, formant une sorte de petit plateau, dont la surface est couverte de vésicules allongées, superposées (sporanges), renfermant 3 ou 4 spores disposées en série linéaire; spores simples. (Coleosporlum Lév. 1. 1.)

3640. U. campanullarum Pers. Op bladen van verschillende Campanula-soorteu. Aest. Aut. (Raben h. 
p. 10; Caeoma campanularum I) z. ct Hi b. Bijdr. I, p. 34) Maastricht, Franq.

3641. U. tusgllaginis Pers. Op bladen van Tussilago farfara en Petasites officinalis. Aest. Aut. (U. fulva c. Tussilaginum Wallr.; Rabenh. p. 12; Caeoma Compransor Dz. et II b. Bijdr. I, p. 34, p. p.) In Herb.: Zutphen, W $t \mathrm{tw}$. (v. v. et s.)

3642. ฮ. ฉareum West. Op bladen van Sonchus arvensis en oleraceus. Aest. Aat. (Coleosporium aureum Bonord.; U. fulva $a$, sonchorum $R$ abenh. p. part. p. 12; Caeoma Compransor Dz, et 11 b. Bijdr. I, p. 34, p. p.)

In Herb.: Spoel bij Culenborg, O u dem. (v. s.)

\section{* Urédinces pourvaes đie Cystides.}

Mycélium ou réceptacle caché sons l'épiderme; spores s'échappant sous forme de poussière. (Lé v. l. l.)

9. Réceptacle composé de très petites cellules formant une sorte de coussinet arrondi, entouré ou parsemé de cystides en forme de massue on de matras, et recouvert de sporanges pédicellés monospores; spores simples conservant quelquefois leurs pédicelles: (Lecythea Lév. 1. 1.)

3643. U. raborum Dec. $O_{p}$ bladen van Rubus caesius en andere Rubus-soorten. Aut. (R a be nh. p. 11; Caeoma Ruborum Dz. et II b. Bijdr. 1, p. 35)

In Herb.: Zutphen, W $t$ w. ex herb. Arkenh. (v. v. et s.)

3644. U. rosae Pers. Op Rozenbladen. Aest. Aut. (Rabenh. p. 11; Caeoma Rosacearum Dz. et II b. Bijdr. I, p. 34)

In Herb.: Leiden, Utrecht, $\mathbf{W} t 1$ w. (v. v. et 8.) Bovendien: Maastricht, Franq. 


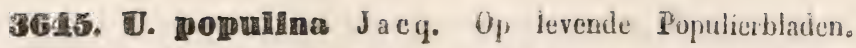
Aest. Aut. (Rabenh. p. 9. Ur. longicapsula $D_{\text {c c }}$; Caeoma cylindricum Dz. et Mb. Bijdr. I, p. 35, p. p.)

In Herb.: Utrecht, W $t$ tw. (จ. v. et s.) - Bovendien: Maastricht, Franq.

3646. U. betulina $W$ ahlen b. $O p$ bladen van Betula alba. Aut. (Ur. populina $b$. Betulae R abenh. p. 9; Caeoma cylindricnm $\mathbf{D} z$. et $\mathbf{l l}$ b. Bijdr. I, p. 35, p. p.)

In Herb.: Utrecht, W $t \mathrm{t}$ w. ( $\nabla$. v. et 8.) - Bovendien: Maastricht, Franq.

3619\%. ש. epitea Kunze. Op bladen van Salix triandra en viminalis. Aut. ( $\mathrm{R}$ abenh. p. 8; Caeoma epiteum Dz. et $\mathrm{II}$ b. Bijdr. I, p. 36)

Naastricht, Franq.

3648. U. poteril Spreng. $0 \mathrm{p}$ bladen van Poterium sanguisorba. Aest. Aut. (R a ben h. p. 8)

In Herb.: Leiderdorp, Dz. et II b. (v. v. et s.)

3649. U. euphorbiae Pers. Op bladen van Euphorbia peplus. Aest. (Rabenh. p. 8; Caeoma euphorbiacearum Dz. et $\mathrm{Mb}$. Bijdr. I, p. 35)

Maastricht, Franq.; Deventer, Cop in herb. Dav.

3650. đ. gyrosa $\mathrm{Rabenh}$. Op bladen van Rubus corylifolius. Aut: (R a benh. p. 9; Caemo gyrosum $\mathbf{D} z$. et Mb. Bijdr. I, p. 35)

In Herb, : Utrecht, W $t \mathrm{tw}$. (v. v. et s.)

10. Réceptacle composé de très petites cellules irrégulières, formant des coussinets charnus, dont la surface est recouverte de cystides pédicellées renflées au sommet, et de cellules cylindriques qui supportent plasieurs spores disposées en chapclet; spores simples isolées, ou encore soudées bout à bout. (Fodlospar rium Lév, 1, 1,)

3651. U. eapracarara bec, Op bladea val Sulia caprata 
un aurita. Aut. (Kaben h. p. 8 ; Cacoma Gaprearum Dz. et $\mathrm{M}$ b. Bijdr. I, p. 36)

In Ilerb.: Utrecht, v. d. S. Lac. (v. v. et $\left.s_{0}\right)-$ Bovendicn: Hlaastricht, Fra nq.

3652. U. accidioldes Dec. Op bladen van Populus alba. Ant. (Rabenh. p. 9; Caeoma aegirinum Dz. et Ml. Bijdr. I, p. 36)

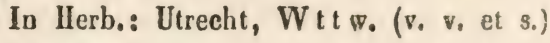

3653. U. Iin $\mathrm{Dec}, \mathrm{Op}_{\mathrm{p}}$ stengels en bladen van Linum catharticum. Aest. (Rabenh. p. 8; Cacoma Lini D z. et II b. Bijdr. I, p. 35)

In Herb.: Zutphen, Wttwo (v. v. et s.) - Borendien: Maastricht, Fran q.

N. B. Speciebus enumeratis accedunt: Caeoma (Uredo) Alliorum Lk., C. Orchidum Lk., C. Artemisiae Lk., C. epigallicum Schlecht., C. Calthae Lk., C. spar.

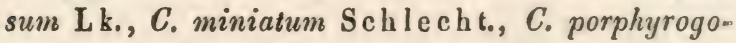
neta Lk., C. mixtum Lk., C. Bypericorum Lk, C. Pseudocyperi Lk., C. Pyrolae Lk. (in $\mathbf{D}$ z. et MIb. Bijdr. I, II, III).

\section{HEOTOMYCES Unger.}

3654. Ix. endogenus Ung. $O p$ stengels van Galium mollugo. Aest. Aut. (Protomyces galii Rabenh. p. 2) In Herb.: Leiden, $\mathbf{D}$ z. et $\mathbf{M}$ bo (v. vo et s.)

\section{UIREIINAIRIA Chev.}

3655. U. vagang $C h e v ; ~ O p$ schors van jonge Elzen. Per annum. (Chov. fl. Par. I. p. 412; Nosophlaca alnea Fr.; Dz. et Mb. Bijdr. I. p. 30)

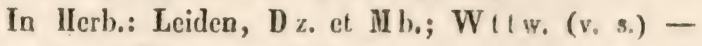
Bovendien: Baastricht, Trauq. 



\section{IN D E X}

Ai) FUNGORUM ENUMERATIONIS PARTEM PRIOREM.

\begin{tabular}{|c|c|c|c|c|c|c|c|c|}
\hline & A. & & & & & & & Pag. \\
\hline & & & & Pag. & Cylindrocolla & : . & . & . .393 \\
\hline Agaricini. & . & . & . . & .303 & Cyphella . . & . . & . & . .307 \\
\hline Agaricus . & 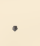 & . & . . & .303 & & & & \\
\hline Amanita. . & . & . & . . & .303 & & 10. & & \\
\hline Armillaria . & . & . & . . & .308 & & & & \\
\hline Ascobolus. & . & - & . . & .396 & Dacrymyces. & . . & . & . . 392 \\
\hline Auricularia & . & - & . . & .391 & Daedalea. . & & . & . .380 \\
\hline & & & & & Discomycetes & . . & . & . . 394 \\
\hline & B. & & & & & & & \\
\hline & & & & & & E. & & \\
\hline Bolbitius. & . & - & . . & .350 & & & & \\
\hline Boletus. & . & - & . . & . 367 & Entoloma & . · & . & . . 331 \\
\hline Bulgaria & . & . & . . & .395 & Exidia . & . . & . & . . 391 \\
\hline & C. & & & & & F. & & \\
\hline Calocera. . & . & . & . . & .392 & Fistulina. & . . & . &.$\quad .382$ \\
\hline Cantharellus & . & . & . . & .362 & & & & \\
\hline Cenangium . & . & . & . . & .405 & & G. & & \\
\hline Clavaria . & . & . & . . & .387 & & & & \\
\hline Clavariei . & . & . & . . & .387 & Galera. . & . . & . & . . 337 \\
\hline Clitocybe & . & , & . . & .313 & Geoglossum. & . . & . & . . 395 \\
\hline Collybia . & . & . & . . & .317 & Gomphidius . & . . & . & . . 354 \\
\hline Coprinus . & . & . & . & .346 & & & & \\
\hline Corticium & . & . & . . & .385 & & $\mathrm{H}$. & & \\
\hline Cortinarius & . & . & . . & .350 & & & & \\
\hline Coryne. & : & . & . & . 392 & Hebeloma . & . & . & . .334 \\
\hline Crepidotus & . & , & . : &, 338 & Helvella. & $\therefore$ & & . 394 \\
\hline
\end{tabular}




\begin{tabular}{|c|c|c|c|c|c|c|c|c|c|c|c|}
\hline Helvellacel & 1 & . & . & & $\begin{array}{r}\text { lag. } \\
394\end{array}$ & Patellaria & . & & & & $\begin{array}{r}\text { Pag. } \\
.405\end{array}$ \\
\hline Hydnei . & . & . & . & . & .382 & Paxillus. & - & . & 。 & . & .353 \\
\hline Hydnum . & . & . . & . & . & .382 & Peziza . & . & . & • & 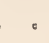 & . 396 \\
\hline Hygrophorus & & . . & . & & .354 & Pezizei . & . & . & . & & . 395 \\
\hline Hymenomye & etes & s. & . & 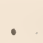 & .303 & Pholiota. & . & . & • & . . & . 332 \\
\hline \multirow[t]{4}{*}{ Hypholoma . } & - & . . & . & . & . 341 & Pleurotus. & . & . & • & . & . 327 \\
\hline & & & & & & Pluteus . & . & . & • & . . & . 330 \\
\hline & & I. & & & & Podisoma & . & . & • & - . & . 393 \\
\hline & & & & & & Polyporei & . & . & 。 & - . & . 367 \\
\hline \multirow[t]{4}{*}{ Irpex. } & . & . . & . & . & . 383 & Polyporus & . & . & . & . & .370 \\
\hline & & & & & & Psalliota & . & 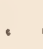 & 。 & . & . 338 \\
\hline & & L. & & & & Psathyra. & . & . & . & . . & .363 \\
\hline & & & & & & ella & . & . & 。 & . . & . 345 \\
\hline Lactarius. & . & - . & . & . & .357 & Psilocybe & . & . & . & , . & .360 \\
\hline Lentinus. & . & . . & . & . & .365 & & & & & 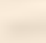 & 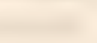 \\
\hline Lenzites . & . & . . & . & . & .366 & & & R. & & 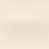 & \\
\hline Leotia . & . . & . . & . & . & 395 & & & & & & \\
\hline \multirow[t]{4}{*}{ Lepiota . } & . & . . & . & . & .305 & Radulum. & . & 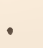 & . & . . & . 383 \\
\hline & & & & & & Russula. & 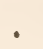 & . & . & . . & .360 \\
\hline & & M. & & & & & & & & & \\
\hline & & & & & & & & S. & & & \\
\hline ius & - & . . & . & . & .362 & & & & & & 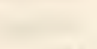 \\
\hline is . & . . & . . & . & . & . 381 & Stereum . & . & - & - & , . & . 384 \\
\hline Mitrula . & . . & - . & . & . & .395 & Stropharia & . & . & 。 & . . & . 360 \\
\hline Morchella & . & . . & . & . & .394 & & & & & & \\
\hline \multirow[t]{2}{*}{ Mycena . } & 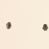 & · $\cdot$ & . & $\because$ & . 320 & & & 'I. & & & \\
\hline & & N & & & & Th & . & & & . & . 383 \\
\hline Naucoria . & . & - . & . & - & . 336 & The & . & . & . & - . & .38 \\
\hline Nolanea . & . & . . & - & . & .331 & Trametes. & . & . & - & & .380 \\
\hline & & 0. & & & & Tremella . & 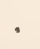 & 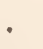 & • & & .391 \\
\hline & & $U$. & & & & Tremellini & & & & . & .35 \\
\hline Omphalia. & - & - . & - & . & . 328 & Tricholoma & - & . & & - . & .30 \\
\hline & & P. & & & & Typhula . & & & & & \\
\hline & . & • & . & & & & & & & & \\
\hline anus. & : & & $\because$ & & & Vol & & & & & \\
\hline
\end{tabular}




\section{IN D E X}

\section{AD IUNGORUM ENUMERA'TIONIS PAR'LLI POSTERIOREM.}

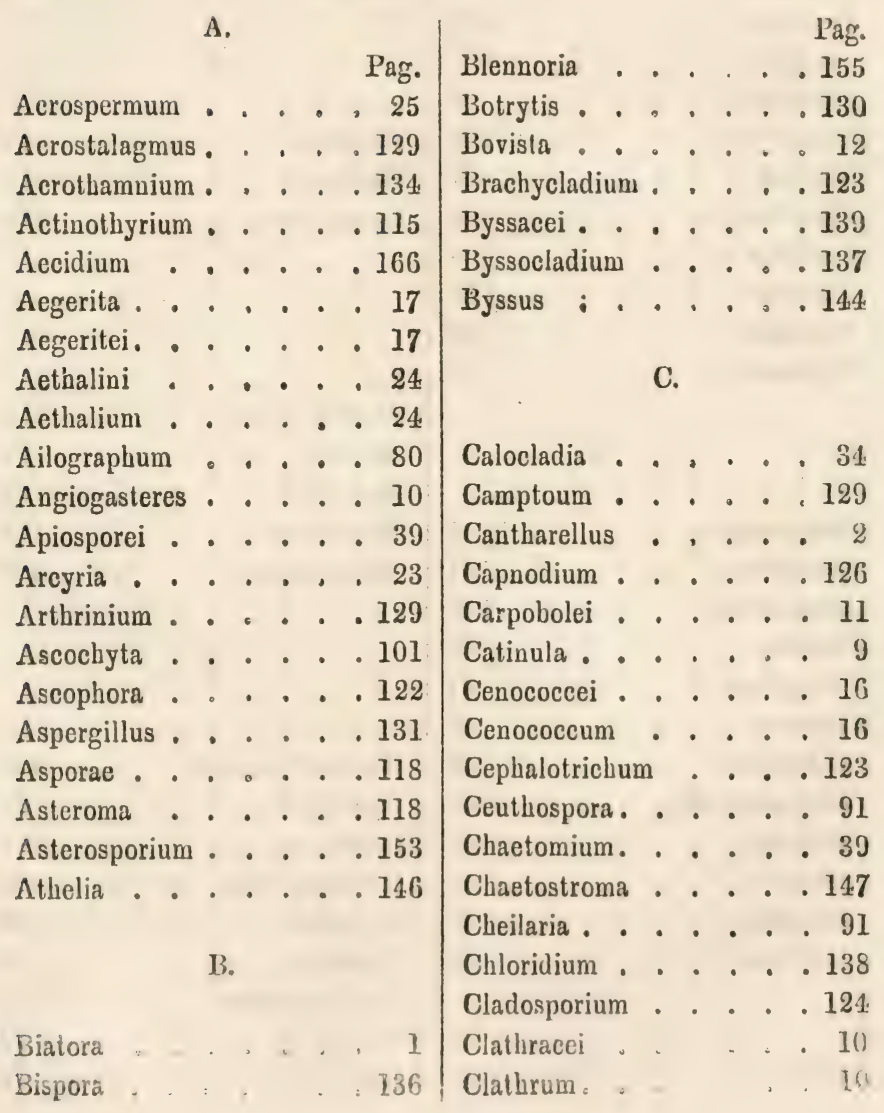


Pag. l'ar.

Clavaria . . . . , 3

Epichysium . . . . 122

Coleosporium . . . 180

Collarium . . . . 139

Epicoccum . . . 147

Colpoma . . . . . 81

Epidochium . . . . 4

Coniomycetes . . . 147

Erineum . . . . . 140

Coniosporium . . . 38

Erysiphe . . . . 35

Exidia . . . . . : 3

Coniothecium . . . . 169

Exosporium . . . . 157

Conisporium . . . . 170

Cordyceps . . . . 40

Eurotium. . . , ..121

Coremium . . . . . 132

Coryneum . . . . 158

Craterium . . . . 19

Cronartium . . . . 166

Cyathus . . . . . 11

Cylindrocolla . . . . 5

Cyphella . . . . . . 3

Cystopus . . . . . . 173

Cytispora . . . . 100

Cytisporei . . . . 82

1).

Eustegia . . . . . 73

F.

Fusarium. . . . . 149

Fusidium. . . . . 155

Fusisporium . . . . 136

G.

Gasteromycetes . . . 10

Geaster . . . . . 12

Geastrides . . . . 12

Dacryomyces . . . . 4

Geotrichum . . . . . 138

Dactylium . . , . . 131

Gloeosporium . . . 150

Destructorei. . . . 143

Diachea . . . . . 20

Dictydium . . . . 21

Diderma . . . . . 18

Didymium . . . . 18

Didymosporium . . . 161

Diplodia . . . . . 84

Discosia . . . . . 84

Dothidea . . . . . 70

Dothidini . . . 69

$\mathrm{F}$

\section{H.}

Helminthosporium . . 127

Hendersonia . . . 82

Himantia. . . . 145

Hormodendrum . . . 135

Hymenula . . . . 152

Hyphelia . . . . 16

Byphomycetes . . . 121

Hysteriacci . . . . 74

Lictostroma .

Hysterinm . 
I. Pag.

Naevia . . . . 8

Illosporium * . . . . 39

Nematogonium . . . . 131

Isariae . . . . . 122

Nidulariacei . . . . . 11

Nostoc . . . . . 2

L.

o.

Lecythea . . . . 181

Leocarpus . . . . 17

Leptostroma . . . . 116

Leptothyrium . . . 117

Libertella . . . $15^{6}$

Lycogala . . . . 2 24

Lycoperdacei . . . . 12

Lycoperdon . . . . . 13

M.

Oidium . . . . . 134

Ozonium . . . . , 144

P.

Palmogloea . . . . 2

Passalora . . . . 126

Penicillium . . . . 132

Perichaena . . . . 22

Periconia. . . . . 123

Marasmius . . . : 3

Peridermium . . . 165

Melampsora . . . . 29

Melanconium . . . 154

Melasmia . . . . 92

Meteorici. . . . . 145

Microbotryum . . . 178

Micropera . . . . 101

Microsticta . . . . 39

Microthyrium . . . 116

Mucedinei . . . . 129

Mucor . . . . . 122

Mucorini . . . . . 121

Mucoroideae : . . . 121

Myxogasteres . . . 17

Myxosporium . . . 157

Myxotrichum . . . 128

Periola . . . . . 147

Perisporei . . . . 30

Perisporium . . . . 38

Peronospora . . . 133

Pestalotia ..... \$4

Peziza . . . . . 5

Phacidiacei . . . . 73

Phacidium . . . . 75

Phalloideae . . . . 10

Phallus . . . . 10

Phlyctena . . . . . 111

Phoma . . . . . 92

Phragmidiacei . . . 157

Phragmidium . . . . 157

Phragmotrichum . . . 160

Phyllactinia . . . . 32

Phylleriacei . . . . 139

Pbyllerium . . . . . 141

Naemaspora. . . . 156 Phyllosticta. . . . 96 


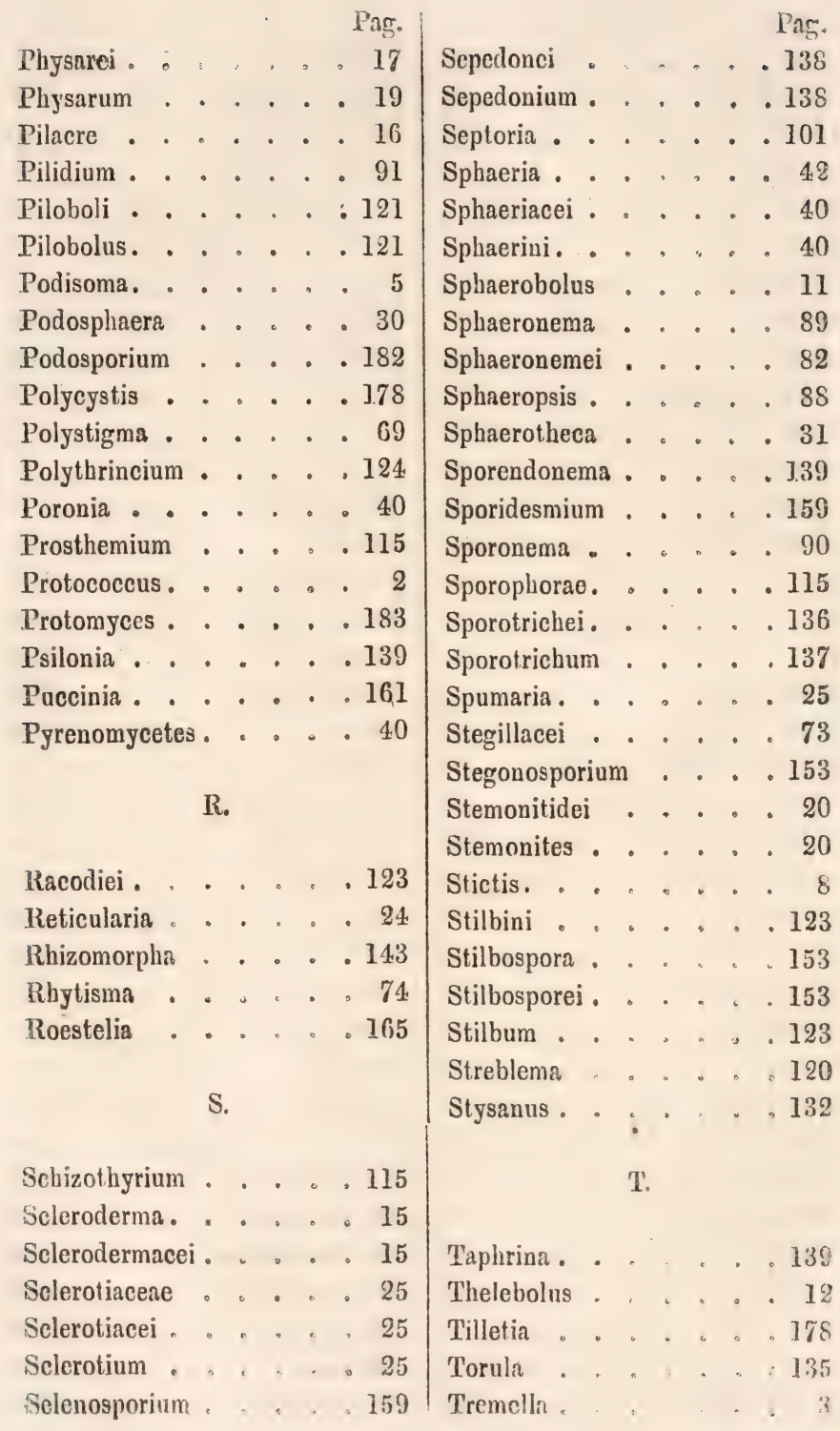




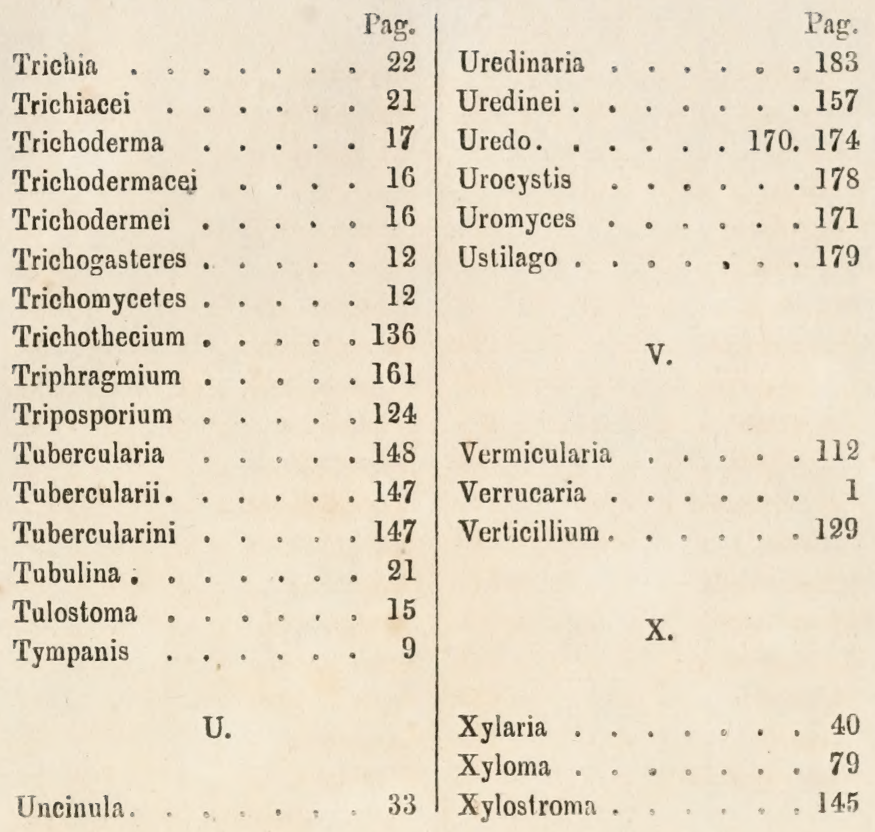



(1) 